\title{
Multistakeholder as Governance Groups: Observations from Case Studies
}

\section{Citation}

Urs Gasser, Ryan Budish \& Sarah Myers West, Multistakeholder as Governance Groups: Observations from Case Studies (Berkman Center Research Publication No. 2015-1, Jan. 14, 2015).

\section{Published Version}

http://cyber.law.harvard.edu/publications/2014/internet_governance

\section{Permanent link}

http://nrs.harvard.edu/urn-3:HUL.InstRepos:16140635

\section{Terms of Use}

This article was downloaded from Harvard University's DASH repository, and is made available under the terms and conditions applicable to Open Access Policy Articles, as set forth at http:// nrs.harvard.edu/urn-3:HUL.InstRepos:dash.current.terms-of-use\#OAP

\section{Share Your Story}

The Harvard community has made this article openly available.

Please share how this access benefits you. Submit a story.

Accessibility 
Research Publication No. 2015-1

January 14, 2015

\title{
Multistakeholder as Governance Groups: Observations from Case Studies
}

\author{
Urs Gasser \\ Ryan Budish \\ Sarah Myers West
}

This paper can be downloaded without charge at:

The Berkman Center for Internet \& Society Research Publication Series: http://cyber.law.harvard.edu/publications/2014/internet governance

The Social Science Research Network Electronic Paper Collection: Available at SSRN: http://ssrn.com/abstract $=2549270$

\footnotetext{
23 Everett Street. Second F1oor - Cambridge, Massachusetts 02138 +1617.495 .7547 • +1617.495.7641 (fax) • http://cyber.law.harvard.edu • cyber@1aw.harvard.edu
} 



\section{ACKNOWLEDGEMENTS}

This synthesis and case study series would not have been possible without the dedication and hard work of many contributors from the Network of Centers (NoC) and the Berkman Center. In particular, we would like to thank our case study authors, who dedicated countless hours to this project and whose efforts were instrumental in its completion. Many thanks also to our friends and collaborators Herbert Burkert, Jens Drolshammer, Raimondo Iemma, Juan Carlos De Martin, Sarah Myers West, Mayte Peters Schomburg, Wolfgang Schulz, Stefaan Verhulst, and Jonathan Zittrain for guidance, collaboration, and support.

We wish to extend our gratitude to Rebekah Heacock Jones, David O'Brien, and Annie Pruitt for conceptual guidance and editorial work, and Gretchen Weber for communications support. Special thanks are due to Ryan Budish for coordinating this research effort and providing extensive research and editorial support.

The Berkman Center is grateful for initial seed funding for this project provided by ICANN, and acknowledges the use of portions of project and general support grants, respectively, by the MacArthur Foundation and Ford Foundation.

\section{Urs Gasser}




\section{Multistakeholder as Governance Groups: Observations from Case Studies}

Urs Gasser, Ryan Budish, and Sarah Myers West

Berkman Center for Internet \& Society at Harvard University 


\section{Table of Contents}

I. Introduction ................................................................................................................................ 1

II. Case Study Process and Overview.......................................................................................... 1

A. Research Process and Methodology .............................................................................................. 1

B. Terminology ........................................................................................................................................ 2

C. Scope............................................................................................................................................... 3

D. Overview of Case Studies ....................................................................................................................... 4

III. Observations on Structural Elements ...................................................................... 8

A. Purpose and Context …………………................................................................................................ 9

1. Purposes of Governance Groups...................................................................................... 9

2. Cultural and Contextual Factors ................................................................................... 10

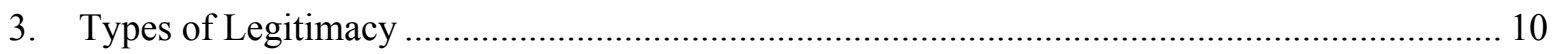

B. Formation ...................................................................................................................................... 11

C. Operation ............................................................................................................................. 12

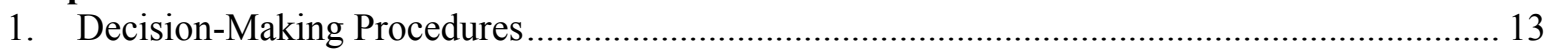

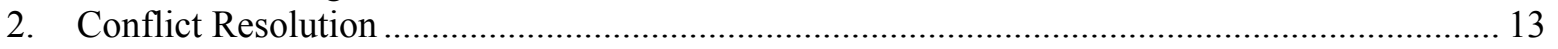

3. Interfaces and Coupling Management .................................................................................... 13

4. Knowledge and Memory Management ..................................................................................... 14

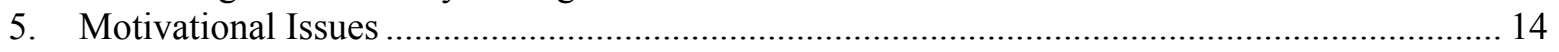

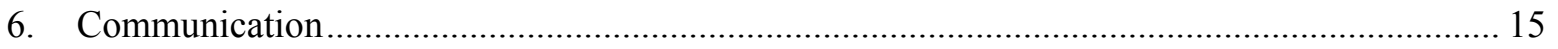

D. Outcomes ........................................................................................................................................... 16

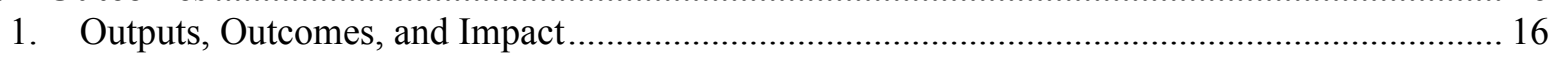

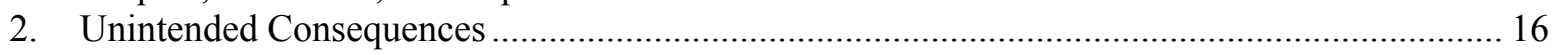

3. Operational Continuity...……………………………………………………………… 17

IV. Critical Success Factors...................................................................................................... 17

A. Inclusiveness ................................................................................................................................. 17

1. Formal Inclusiveness: Identifying and Including Stakeholders................................................. 18

2. Voting, Consensus, and the Use of Decision-Making Processes................................................ 19

3. Effect of Alternative Participatory Mechanisms..................................................................... 20

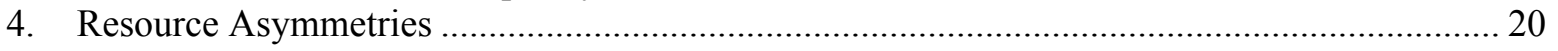

B. Transparency ............................................................................................................................................. 21

C. Accountability ...................................................................................................................................... 22

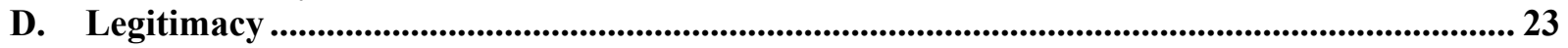

E. Effectiveness...................................................................................................................................... 24

V. Summary of Observations .................................................................................................. 25

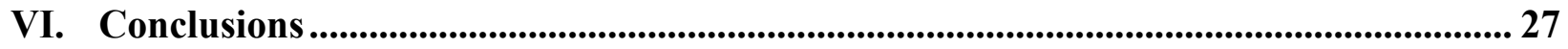

VII. Appendixes A-L: Case Studies.....................................................30 


\section{Introduction}

This paper synthesizes a set of twelve case studies of real-world governance structures. ${ }^{1}$ Together with the underlying case studies, it is the result of a globally coordinated, independent academic research pilot project by the Global Network of Interdisciplinary Internet \& Society Research Centers (NoC). Facilitated by the Berkman Center for Internet \& Society at Harvard University, this study examines existing multistakeholder governance groups with the goal of informing the evolution of - and current debate around - the future evolution of the Internet governance ecosystem in light of the NETmundial Principles and Roadmap, discussions at the Internet Governance Forum, and the NETmundial Initiative, as well as other forums, panels, and committees.

Internet governance is an increasingly complex concept that operates at multiple levels and in different dimensions, making it necessary to have a better understanding of both how multistakeholder governance groups operate and how they best achieve their goals. With this need in mind, at a point where the future of Internet governance is being re-envisioned, this project aims to deepen our understanding of the formation, operation, and critical success factors of governance groups (and even challenge conventional thinking) by studying a geographically and topically diverse set of local, national, and international governance models, components, and mechanisms from within and outside of the sphere of Internet governance, with a focus on lessons learned.

The research effort is grounded in a diversity of global perspectives and collaborative research techniques. Adhering to objective and independent academic standards, it aspires to be useful, actionable, and timely for policymakers and stakeholders. More broadly, the Network of Centers seeks to contribute to a more generalized vision and longer-term strategy for academia regarding its roles in research, facilitation and convening, and education in and communication about the Internet age.

\section{Case Study Process and Overview}

\section{A. Research Process and Methodology}

Responding to the dynamics of the current debate about the future of Internet governance, the objectives of this pilot project have evolved over time as we have gained a better understanding about the interaction between the formation and operation of governance groups and the contextual environments in which such groups operate.

Initially drawing upon the Panel on Global Internet Cooperation and Governance and Governance Mechanisms ("Panel Report"), ${ }^{2}$ the research began with the guiding question of whether a set of best practices that built off of the Panel Report could be distilled from a series of select real-world case studies examining various governance groups. In that mode we sought case studies that would help us better understand three components of the Panel Report: (1) how governance groups best match challenges with the organizations, experts, networks, and governing bodies/entities most able to help develop legitimate, effective, and efficient solutions; (2) how to structure the flow of information and knowledge necessary for successful governance; and (3) how different governance groups approach coordination between regional and global (or national and regional) governance networks in order to avoid conflicting directives. 
Building upon twelve case studies, this synthesis concludes that there is no single best-fit model for multistakeholder governance groups that can be applied in all instances. Rather, it reveals a range of approaches, mechanisms, and tools available for both the formation and operation of such groups. The analysis demonstrates that whether governance groups meet their objectives depends to a large degree on the careful selection, deployment, and management of suitable instruments from this "toolbox." As governance groups pass through different phases of operation, conveners and facilitators must remain alert to changes in circumstances that necessitate adjustments to the approaches, mechanisms, and tools that they deploy in order to address evolving challenges from inside and from outside. The case study series provides insights into how those instruments can be deployed and adjusted over time within such groups, and highlights how important contextual factors they interact with may be successfully managed within given resource restraints.

The case studies come from the global academic institutions that constitute the Network of Centers. After a series of planning meetings and learning calls among the participating Centers, the research team met in May 2014 to collaboratively identify and select case studies based on a set of criteria emerging from the current debates about the future of Internet governance, as described above. The case studies (described in greater detail below) represent a wide variety of initiatives, including public and private sector efforts, operating at national and international levels in regions all over the world, addressing issues with varying levels of technical complexity. ${ }^{3}$ The selected case studies also cover a wide range of activities, including drafting legislation, developing policy in the absence of formal regulatory authority, defining and exploring the scope of a problem, and building connections among stakeholders. While some of the case studies come from areas related to Internet governance, others come from outside the Internet governance ecosystem. Although they differ in many respects, the governance groups documented in the case studies share a common commitment to using multistakeholder processes to solve complex issues.

Each of the case study authors conducted desk research and interviews in order to develop the case studies. In several cases, the case studies reflect direct input from participants and leaders in the group's operation. Once drafted, the case studies underwent several rounds of peer review and served as key inputs for a meeting of the Network of Centers on October 1-2, 2014. At the meeting, the case studies were discussed and key findings were identified and synthesized. ${ }^{4}$ That discussion formed the basis of this synthesis paper.

\section{B. Terminology}

At the outset of this research, we conducted a review of existing literature to inform our analysis of the cases. In particular, our review focused on the rich literature on multistakeholder governance, examining the body of scholarship that provides frameworks for, or definitions and critiques of, the term. In its most basic interpretation, multistakeholder governance implies the incorporation of representatives from multiple groups in discussions and decision making. ${ }^{5}$ Multistakeholder partnerships can, in their idealized form, facilitate global governance by bringing in a range of resources and competencies to address common problems. ${ }^{6}$

In the context of Internet governance the multistakeholder approach has taken on greater salience since the World Summit on the Information Society (WSIS). Alongside the rise in prominence of non-state actors in global affairs, the UN resolution providing for WSIS provided an initial legal 
basis for multistakeholder participation in Internet governance discussions. As initially used, the term "multistakeholder" was associated with private-sector leadership, but its definition was somewhat vague. By the Tunis Agenda its meaning became more consolidated to mean incorporation of governments, business entities, civil society and intergovernmental organizations. ${ }^{7}$ The Internet Governance Forum (IGF) further expanded the scope of stakeholder representation to include the academic and technical communities.

Though the literature reflects general agreement on core principles, it also suggests some divergence as to the processes that constitute multistakeholder governance, particularly across different disciplinary perspectives. Governance approaches come in many shapes and sizes: in addition to multistakeholder, governance processes may alternately be described as distributed, decentralized, or collaborative. ${ }^{8}$ They also may differ depending on the governance context: governance in the economic domain may look quite different and operate at different scalar levels than governance in the environmental domain. Thus, while the most basic definition of multistakeholder governance is generally agreed upon at the core, understandings of governance practices are shaped by a variety of interpretations and perspectives at the periphery. ${ }^{9}$

Given this lack of consensus, we elected not to take a definitional approach to the work; we did not try to match our case studies onto predefined templates of how a governance group should look, nor did we focus only on those elements that aligned with a preselected definition. Instead, we sought to contribute to our shared understanding by taking a phenomenological approach and focusing on the functions of the groups as well as the elements critical to those functions. One benefit of this bottom-up approach, which is particularly relevant in the context of the global research design of this study, is that it accommodates the varying translations of the term "multistakeholder" into different languages, in which it frequently takes on different nuances and connotations.

\section{Scope}

This project takes a networked approach to academic research. By activating a global network of interdisciplinary research centers, we were able to conduct in parallel research on twelve different case studies, and then collaboratively consider lessons from those case studies. Although the work was conducted in a highly compressed timeframe, we endeavored to demonstrate that such an interdisciplinary and global approach can offer nuanced, helpful, and timely insights to the stakeholders involved in Internet governance debates or concerned with the implementation of governance groups more broadly.

Of course, such an approach is not without methodological limits. First, our conclusions are bounded by the samples chosen. We chose only cases that were multistakeholder in whole or in part, and did not study groups with entirely different structures. Nor did we seek examples of failed governance groups; although not all of the groups analyzed in our research achieved all of their intended goals, they all were viable in their operations and sustainable throughout their existence. Accordingly, this research does not endeavor to identify factors that may trigger failure and instead focuses on factors that seem to enable success. Second, as with most case study-centered research, this project does not assert to be a statistically representative sample of models, regions, or experiences. Despite these limitations, we believe this synthesis and the accompanying case studies provide timely insights to the current debate over Internet 
governance, while also laying a foundation for future research that will be able to more fully explore the concepts, considerations, and questions raised in this work.

\section{Overview of Case Studies}

This synthesis paper and its foundational case studies are not standalone documents, but are intended to be read as interacting elements. For the convenience of readers, below we provide short abstracts for each case study; we recommend reading the relevant case studies in their entirety where further information is necessary.

Aviation - The Worldwide Slot Guidelines - Giovanni Sartor and Hanna Schebesta, European University Institute ("Aviation Slotting"): ${ }^{10}$ This case study explores the Worldwide Slot Guidelines (WSG), which represent a set of agreements made under the auspices of the International Air Transport Association (IATA) in order to allocate airport capacity. The right to use airport capacity for the purpose of takeoff and landing operations at airports is commonly called slots, with each airport having a finite amount of airport capacity. Thus, the slot allocation process is one of resource management: the WSG were developed as a global industry standard to address a key constraint in the aviation domain. The actual process of slot allocation is complex and involves organizations and stakeholders at multiple levels, all falling under the umbrella of IATA, a trade association representing 240 airlines. It designates particularly congested airports, creates roles of airport coordinators, and establishes management principles for slot allocation. Because they manage a valuable economic resource, the transparency and independence of coordinators is of particular concern: this has resulted in the institutionalization of a negotiating process to ensure accountability among key stakeholders. Through an examination of the history and key points of change in the process, the WSG case presents an example of private sector coordinated resource management.

The Evolution of Governance Structure in Cryptocurrencies and the Emergence of CodeBased Arbitration in Bitcoin - Markus Oermann and Nils Tollner, Hans-Bredow-Institute ("Bitcoin"): "11 This case study describes the process by which Bitcoin revised its core code to accommodate a new feature called "multi-signature transactions." Bitcoin is a cryptocurrency, which was introduced in 2009 and has spread rapidly since then. It is based on open-source peer-to-peer software that that leverages a network of user accounts (wallets) set up on peripheral sites in which the units of account (Bitcoins) may be stored after they are produced and transmitted. It is a goal of cryptocurrencies in general to operate without a central agent, which makes it complicated to resolve disputes. To address this, Bitcoin added multi-signature transactions. This case study examines how that change was made. In particular the case study explores how an open source community is able to maintain a stable codebase that can serve as a basis for an entire form of currency, while still making necessary changes. The case shows that the decision-making processes regarding transformations of Bitcoin's governance structure are not as transparent as one might expect given the cryptocurrency's commitment to open source. However, major changes to the code are publicly discussed in the Bitcoin developers' community and the authors could not identify a single case of a decision in which the core development team deviated from the consensus of the community.

Creative Commons (CC) - Federico Morando, Nexa Center for Internet \& Society at Politecnico di Torino ("Creative Commons"): ${ }^{12}$ This case study examines the formal structure 
of Creative Commons (CC) as an organization stewarding the development of the most wellknown and widespread set of standard public copyright licenses. In particular, the case study explores the interaction of $\mathrm{CC}$ with its own community and with a broader set of international stakeholders during the drafting of the fourth version of the Creative Commons Public Licenses, which took place between mid-2011 (with public discussion starting in November 2011) and November 2013. This case study highlights how a transnational organization can operate outside of the scope of formal regulatory authority, but with the purpose of creating an output (CC licenses) that have legal weight. In such an environment credibility and legitimacy are paramount, necessitating opportunities for public consultation, transparency, and even admitting mistakes.

Fighting Spam the Multistakeholder Way - A Case Study on the Port 25/TCP Management in the Brazilian Internet - Ronaldo Lemos, Carlos Affonso de Souza, Fabro Steibel, Juliana Nolasco, Institute for Technology and Society at Rio de Janeiro State University ("CTSpam"): ${ }^{13}$ This case study explores how CGI.br, the multistakeholder Brazilian Internet Steering Committee, addressed through a collaborative decision making and educational process the difficult issue of spam propagation. Although the technical solution to the spam problem in Brazil was relatively clear, convincing stakeholders to adopt the solution was challenging. Telecommunications companies and ISPs initially resisted this recommendation out of concern around the costs of switching and the challenges of communicating the change to end users. This case study examines how a multistakeholder process involving telecommunications companies, ISPs, consumer rights associations, and government ministries and agencies was able to overcome these obstacles while simultaneously respecting consumer rights, freedom of speech, and commercial competition. CGI.br's Anti-Spam Working Commission (CT-Spam Commission) highlights how collaborative governance can be applied in an iterative and educational fashion. Addressing the spam issue would require buy-in and cooperation from a variety of parties, and by engaging all stakeholders, the CT-Spam Commission was able to identify the concerns of stakeholders and then develop a variety of educational materials, technical reports, and policy changes in order to address those concerns. By developing the policy in such a fashion, the CT-Spam Commission was able to gain the support of the telecommunications companies and ISPs without regulatory oversight. Ultimately, with the buyin of key stakeholders, implementation of the Port 25/TCP recommendation in 2013 led to a dramatic decrease in spam in Brazil.

Enquete-Kommission Internet und digitale Gesellschaft (Enquete Commission on Internet and Digital Society) - Kirsten Gollatz, Sarah Herweg, Jeanette Hofmann, Humboldt Institute for Internet and Society ("EIDG"): ${ }^{14}$ This case study explores the Enquete Commission on Internet and Digital Society (EIDG Commission), which is a parliamentary inquiry body of the German Bundestag that conducted its work from May 2010 until April 2013. Enquete Commissions are special bodies of the German Bundestag, which form an interface between policy-makers and academic and professional experts to examine through discussion forums and working groups broad and complex matters of society that cannot be sufficiently dealt with within the regular parliamentary framework. The Commission was introduced in response to rising awareness regarding the challenges for politics and society that digitization brings along, which became apparent in a controversial draft bill before the parliament that called for the mandatory blocking of websites with child pornography-related content. The Commission consisted of 34 individuals, 17 democratically-elected members (members of 
parliament) and 17 experts and practitioners representing industry, trade unions, civil society and academia. Furthermore, the public was invited to participate in the Commission's consultations "to a special degree," which included efforts at transparency and an online engagement platform. It is too early to assess the policy impact of the Commission's work, as it is still unclear to what extent the new parliament will include the Commission's policy recommendations in its legislative initiatives. However, as a mechanism for bringing together heterogeneous sources of knowledge and expertise for the purpose of enlightening decision-making processes, the general model of an Enquete Commission can be regarded as an effective and legitimate mechanism for parliaments and possibly other deliberative bodies. Going forward, finding the appropriate implementation mechanisms that will help inform evidence-based policy making and provide justification for spending further resources should be taken into account in the process design.

Multistakeholder Approaches to Water Resource Management in the White Volta River Basin - Rebekah Heacock Jones, Ryan Budish, et al, Berkman Center for Internet \& Society ("White Volta"): ${ }^{15}$ This case study examines the deployment of the decentralized integrated water resource management (IWRM) model for the management of water resources at both the local and transboundary levels of the White Volta River Basin. The Volta River Basin spans across six countries-Benin, Burkina Faso, Cote d'Ivoire, Ghana, Mali, and Togo. One of the Basin's four main sub-basins is the White Volta in Northern Ghana. The case study investigates and compares governance at the sub-basin level through White Volta Basin Board (WVBB) and at the Basin level through the Project for Improving Water Governance in the Volta River Basin (PAGEV). It also discusses the development of IWRM in water governance more broadly. The case study concludes that PAGEV was more successful in implementing IWRM principles than the WVBB, but questions remain as to their decision-making processes and the full extent of stakeholder participation.

Multistakeholder Governance and Nodal Authority - Understanding Internet Exchange Points - Ben Wagner and Patricia Mindus, European University Institute ("IXP"): ${ }^{16}$ This case study considers Internet exchange points (IXPs) as an example of governance processes in action. Internet exchange points are the points of connection between different Internet networks, which enable different networks to exchange traffic at a shared facility without cost to either party through a process known as "peering". Three different IXP governance models representing large and influential IXPs are compared: the DE-CIX in Frankfurt, CAIX in Cairo, and KIXP in Nairobi. DE-CIX, the largest IXP in the world, is a subsidiary of the German Internet trade association eco, and is thus "owned" by the Internet industry in Germany. Though well functioning, this has meant that key stakeholder groups such as civil society, and the academic and technical communities are excluded from participating in discussions over policy decisions. In contrast, the Cairo Internet Exchange Point (CAIX) is run by a public authority, the Egyptian Ministry of Communications and Information Technology. Though it is governed by a broad set of stakeholders including private sector, government, and civil society representatives, its decision-making processes are somewhat opaque. Lastly, KIXP was founded by a Kenyan network engineer and is governed by a local trade association. While set up with multistakeholder coordination under the leadership of the private sector, its day-to-day operations and governance fall under private sector control. By tracing out the plurality of models used for IXP governance and comparing the processes of developing peering relationships, this case provides unique lessons for the governance process, particularly surrounding trade-offs between inclusiveness and effectiveness. 
Towards a Cyber Security Policy Model - Israel National Cyber Bureau (INCB) Case Study - Daniel Benoliel, Haifa Center of Law and Technology (HCLT), University of Haifa Faculty of Law ("INCB"): ${ }^{17}$ This case study examines the elements of the creation of a cyber security policy model at a national level. It uses Israel's recently established National Cyber Bureau (INCB) cyber command funneled by its national cyber policy as a case in point. In so doing the brief offers a cross-section comparison between leading cyber security national policies of the United States, United Kingdom, Canada, Japan and the Netherlands. It further introduces comparable policies including the balancing of cyber security with civil liberties, cyber crime policy, adherence to international law and international humanitarian law, forms of regulation (technological standards, legislation, courts, markets or norms) and prevalent forms of cooperation (intra-governmental, regional, public-private platform (PPP) and inter-governmental cooperation).

A Bill of Rights for the Brazilian Internet ("Marco Civil") - A Multistakeholder Policymaking Case - Ronaldo Lemos, Carlos Affonso de Souza, Fabro Steibel, Juliana Nolasco, Institute for Technology and Society at Rio de Janeiro State University ("Marco Civil"): ${ }^{18}$ This case study explores the events that led to the passing of the "Marco Civil da Internet" legislation, which became law in Brazil in April 2014. The first of its kind anywhere in the world, Marco Civil is a landmark "Bill of Rights" for the Brazilian Internet users. The legislation is unique both due to its substance and the process by which it was created. From a substantive standpoint, Marco Civil applies to the Internet key principles such as freedom of expression, net neutrality, and due process. But perhaps more important than the substance is the way in which the bill was drafted and enacted. This case study explores the unique features of that drafting process. Marco Civil was conceptualized in 2007 and, over the course of many years, was drafted using an open multistakeholder process through which members of the public, government, global and local Internet companies, civil society, and others engaged in negotiations over the legislation's text, largely mediated through online platforms. Although the law was ultimately adopted, the process was not without its challenges. For example, attracting early contributors and participants was difficult, as was debating contentious topics openly among the stakeholders, who frequently disagreed. After many delays, Marco Civil was eventually passed. This case study will explore the factors that enabled a complex piece of legislation to be drafted with input and consultation from a distributed and diverse collection of stakeholders.

The Global Multistakeholder Meeting on the Future of Internet Governance (NETmundial) - Marília Maciel, Nicolo Zingales, and Daniel Fink, Center for Technology \& Society at FGV School of Law, Rio de Janeiro ("NETmundial"): ${ }^{19}$ This case study examines NETmundial, the Global Multistakeholder Meeting on the Future of Internet Governance, which was held in Sao Paulo, Brazil on April 23-24, 2014. The meeting was convened by 1net, a coalition of stakeholder groups involved in Internet governance discussions, in partnership with the Brazilian Internet Steering Committee (CGI.br), in response to revelations of mass surveillance of communications by the United States. It sought to develop a set of universallyacceptable Internet governance principles as well as a way forward for the evolution of the Internet governance system, which together could serve as a framework for the governance and use of the Internet. It convened 930 participants from 110 different countries, representing civil society, the private sector, academia, the technical community, governments and intergovernmental organizations, as well as over 1000 remote participants from 23 countries 
around the globe. It also employed a content contribution platform that sought to crowdsource inputs from stakeholders for the production of the outcome document. The meeting served as a demonstration of the multistakeholder process in action: in the production of the outcome document, stakeholders with a diverse range of backgrounds and interests collectively negotiated the inclusion or exclusion of highly-sensitive and complex issues. While the process of achieving rough consensus involved sometimes messy debates and there were procedural imperfections, the case is informative for its structured production of bottom-up multistakeholder outcomes.

Swiss ComCom FTTH Roundtable Case Study - Urs Gasser, Ryan Budish, David R. O'Brien, et al., Berkman Center for Internet \& Society ("Swiss ComCom"): ${ }^{20}$ This case study explores the dimensions of how the Swiss government-in particular, the Swiss Federal Communications Commission (ComCom) and the Swiss Federal Office of Communications (OFCOM) - used a multistakeholder process to organize private sector firms to begin deploying in a coordinated fashion a fiber optic network connected to every home in Switzerland. Although fiber optic connections were available in some areas of Switzerland, telecommunications and cable companies in many regions of the country had not yet upgraded older network infrastructure to fiber or connected it to homes. The large capital investments required, competing interests among stakeholders, and the lack of coordinated efforts to serve common interests were all contributing factors. The Swiss government was eager to see the national infrastructure upgraded, but lacked the formal authority to regulate the fiber optic networks under its communications laws. In lieu of regulation, the Swiss government convened a series of voluntary roundtable meetings between 2008-2012 to achieve a consensus-based solution. The Roundtables, and related technical working groups, attempted to bring together the critical stakeholders and surface the key issues that needed to be resolved. Using Swiss public records, reports, and personal accounts of those directly involved, this case study examines the roles and approaches used in the Roundtables and evaluates the process used as a model of ad hoc, problem solving and distributed governance.

Turkish Internet Improvement Board - Leyla Keser, Mehmet Bedii Kaya, IT Law Institute, Istanbul Bilgi University ("TIIB"): ${ }^{21}$ This case study focuses on a multistakeholder working group that the Turkish Internet Improvement Board created in order to generate innovative, bottom-up approaches for fixing the Internet law in Turkey. In 2007, Turkey adopted its first comprehensive Internet content regulation legislation: Law 5651, the "Regulation of Publications on the Internet and Combating Crimes Committed by Means of Such Publications." Since its adoption, Law 5651 has come under scrutiny for enabling overly broad restrictions on web content, its establishment of criminal liability for hosting providers who fail to adequately restrict content, the threats it posed to Internet-related innovation in the country, the cybersecurity vulnerabilities that resulted from the widespread use of circumvention tools by Turkish Internet users in response to content restrictions under the law, and related data protection concerns. The law also called for the creation of the Internet Improvement Board. In 2011, the Board created the "5651 Working Group," comprised of seven representatives from government, civil society, academia, and the private sector, to explore possible solutions to these problems. This case study examines how the working group collaborated with a broader group of stakeholders to develop a resolution, delivered to the Ministry of Transportation, Maritime Affairs, and Communication, proposing amendments to Law 5651.

\section{Observations on Structural Elements}


In this section, we explore the key structural elements of these case studies, trace through some of the nuanced differences that distinguish them, and identify areas they have in common. We conduct this evaluation across several dimensions, which emerged "bottom-up" from the crosssectional analysis and discussion of the cases (as further described in the Methodology section above). First, we examine some of the many reasons multistakeholder groups are formed. Second, we describe the process of formation, and the ways in which the convening process and initial establishment of the groups can have downstream effects on their subsequent operation and outcomes. Third, we analyze some of the key aspects of their operation, including how they approach decision-making, conflict resolution, and internal and external communications. Lastly, we discuss their outcomes (both intended and unintended), how they sought to measure their success, and their continuing impacts after their formal operations concluded.

\section{A. Purpose and Context}

\section{Purposes of Governance Groups}

The case studies reveal diverse motivations drove the formation of governance groups, ranging from supplementing existing institutions and drafting legislation to more open-ended approaches that seek to define or inform the scope of an issue.

In several cases, governance groups were created to help advise or inform existing political institutions in order to help supplement a perceived deficiency in the process. For example, in the Marco Civil, TIIB, and EIDG case studies, multistakeholder bodies were created as a response to controversial legislation. The groups in each of those cases were intended to help bring specialized knowledge to bear on the legislative process with the ultimate aim of informing legislative bodies and drafting legislation.

Governance groups can play an important executive role in carrying out policies. For example, in the INCB case study, multiple actors joined together in the execution of cybersecurity policy. Stakeholder involvement at the implementation stage provides an opportunity for the actors that may be affected by a policy to shape its execution.

In contrast, in other case studies the governance groups were intended not merely to supplement or support, but to replace existing institutions or fill in gaps between institutional authorities. For example, the Bitcoin, NETmundial, and Creative Commons case studies all describe governance groups that operate outside of the domain of existing institutions. In fact, as the Bitcoin case study describes, the primary goal of the Bitcoin developers was to create an alternative currency that was specifically not dependent on existing institutions.

Within the case studies there are other, less explicit but nonetheless important purposes driving the formation of governance groups. For instance, we see examples of more open-ended purposes such as building networks and relationships between partners or advancing knowledge about an issue. Although not the primary purpose of the group, the Creative Commons case study describes the important goal of helping to build a global network among knowledgeable, interconnected individuals. Similarly, governance groups can allow members to develop common shared definitions and understandings, overcoming language barriers that frequently develop among members from different domains. This may help facilitate greater understanding between partners, reduce the potential for conflict, and smooth the way forward in tackling complex issues. 


\section{Cultural and Contextual Factors}

A key thread throughout this paper is the importance of cultural and contextual factors in shaping the functioning and the outcome of governance groups. Of particular importance seem to be: preexisting relationships between stakeholders, the relationship between the governance group and governmental institution, the allocation of resources, and geopolitical factors.

How the group is situated within existing institutions or normative structures can have downstream effects on the relationships between stakeholders and the results inside the governance group. For example, in the EIDG case study, the Commission existed within a parliamentary framework and was made up of both members of parliament and outside experts. The members of parliament knew each other as repeat players and partners within the political system, and most expert representatives were selected on the basis of their political party affiliation. The fact that the participants' relationships were largely defined by politics ultimately made the Commission itself more political.

The case studies demonstrate a range of different relationships between government and governance groups. This relationship may differ depending on the historical and political context of the issues involved. Because cybersecurity directly implicates national security interests, the INCB in Israel illustrated much stronger governmental control of both the structure of the process and eventual outcomes. Governance groups acting in areas in which government has not traditionally held a strong position may include less government involvement (NETmundial, for example).

The allocation of resources (including time) inhibited full participation of some stakeholders in several case studies. For example, at NETmundial, a lack of clarity regarding the availability of funds until late in the process meant that some potential participants were deterred from attending the meeting in person. Similarly, a lack of both time and funding at NETmundial limited the ability of the organizers to offer multilingual support during the online consultation period, limiting participation to only those who were proficient in English.

Geopolitical factors and crises influenced the governance groups in a number of the case studies. For example, the Snowden disclosures prompted the formation of NETmundial and added new urgency to the passage of the Marco Civil. And, as noted previously, several of the governance groups were prompted by controversial legislation.

\section{Types of Legitimacy}

Legitimacy is an important aspect of governance groups because it helps explain why a group's decisions might be adopted and followed even in the absence of formal enforcement mechanisms. Some governance groups utilize traditional mechanisms of legitimation, including legal frameworks, checks and balances, and voting processes. However, other groups choose to (or must) seek their sources of legitimacy elsewhere.

Interestingly, most governance groups in the case studies appear to rely upon multiple and overlapping sources of legitimacy, and move between them depending on the circumstances and context. For example, the EIDG not only used traditional sources of legitimacy derived from the German constitutional framework providing for Enquete commissions, but also generated expertise-based and procedural legitimacy through its knowledge creation process, which included open meetings and opportunities for public feedback. It is important to note, however, 
that governmental-based legitimacy for a governance group is derived from the legitimacy of the government itself; in other words, a government that is not viewed as legitimate in its exercise of power, cannot confer legitimacy upon a governance group merely by association. Moreover, this sense of legitimacy is entirely perspective-dependent, as different groups may have different assessments of a government's legitimacy and thus different assessments of an affiliated governance group's legitimacy.

Legitimacy can manifest through input legitimacy or output legitimacy. Input legitimacy refers to perceptions of a fair process: for example, the involvement of a wide range of stakeholders on equal footing, and with a shared understanding of the procedural rules, aided the input legitimacy of the CT-Spam group and the NETmundial conference through the inclusion of a wider range of viewpoints. By contrast, other cases illustrated the role of output legitimacy, in which the quality of the output of a process ensures it is seen as legitimate among stakeholders. The coordinated and efficient deployment of FTTH throughout Switzerland has meant that the ComCom roundtables were largely seen as a success, despite the fact that some stakeholders claimed the process disenfranchised them.

Lastly, context proved important in determining the centrality of legitimacy. We found that legitimacy becomes most important when conflicts arise during the multistakeholder process. In these instances, the groups had to assert stronger forms of legitimacy. Where there was broad approval of the process and its objectives, "lighter" forms of legitimacy tended to be more accepted.

\section{B. Formation}

The architecture of the groups examined in the case studies varied notably, despite the fact that they all shared a multistakeholder approach. Each group interpreted and implemented this approach in highly contextualized ways. These choices have consequences for the decisionmaking process and eventual outcomes.

The way in which multistakeholder groups are convened can have important effects on the ultimate outcome of the process. For example, in the TIIB case, the Turkish Internet Improvement Board was authorized by law to create a multistakeholder group in order to research issues relating to the widely criticized law and propose amendments to it. Though in many ways the group was intended to form a critique of the law, its formation necessarily predicated close involvement of the government, which may have challenged its legitimacy. The CT-Spam group was formed in the wake of a failure to convince key stakeholders of the importance of an issue, which allowed the issue to become a crisis. Because of this, a multistakeholder approach proved to be the only way to develop a sustainable solution to a critical infrastructure problem Brazil was facing.

The composition of the group is a key element of the formation process. The case studies illustrate a wide variety of approaches: some sought to obtain as broad-based participation as possible through voluntary processes, while others were more selective in choosing representatives of stakeholder groups to participate in more intimate discussions. On the far end of the spectrum, the NETmundial process incorporated over 930 attendees and over 1,000 participants who joined the meeting through remote hubs. On the other end, ComCom selected a small group of members to participate in its round tables, seeking a balance between enough 
participants so that decisions could be broadly accepted but not so many participants that the process would take too long. In each case, the breadth of participation was calibrated to fit the issue at hand.

Inclusion criteria influence who may be considered a potential participant or stakeholder. ComCom limited participation in the roundtables to only those groups that had already begun the process of making investments in FTTH, and furthermore only allowed CEOs to serve as representatives of these groups. Similarly, participation in the airport slotting guidelines development process is inherently limited to the small group that can own major airports or airlines, and only the delegates from these stakeholders with authority to make decisions on their behalf are allowed to participate in the slotting process at the bi-annual Slot Conferences. These restrictive inclusion criteria ensured participants in these highly technical matters had the necessary expertise and ability to make informed, binding decisions. However, they limited the diversity of voices in the process, which may have inhibited the creativity of outcomes. Additionally, it is worth noting that the transparency of the inclusion criteria and the identity of the individual or organization setting such criteria may also affect the perceived legitimacy of the group as a whole.

While all the case studies examine groups that can be called multistakeholder, the groups demonstrate a diverse range of structures and internal architectures. Some groups created topical divisions, segmenting off more technical issues into working groups that informed broad-based plenary processes. For example, the EIDG created twelve working groups to produce reports on a variety of topics. These reports were later discussed and voted on in plenary sessions. Others created participatory divisions. In addition to general participation at NETmundial, organizers created several committees that helped to structure and operate the event, including the Executive Multistakeholder Committee, which shaped the ultimate outcome document. At the meeting itself, participants were categorized by stakeholder group, and through rotation of microphones each group was given equal voice regardless of the total number of participants it had. Finally, some groups had no hierarchies whatsoever. During the drafting stage for the Marco Civil legislation, for instance, there was no clear established hierarchical structure for participation; rather, an open platform channeled public conversation in a productive manner, enabling the legislation to be drafted in a bottom-up fashion.

\section{Operation}

The case studies describe not just the structure of the multistakeholder organizations, but also their operation in addressing a variety of complex issues. Although all of the case studies reflect collaborative and distributed attempts to address a specific problem or set of problems, the mode in which each group operated and functioned in order to address that problem differed. This section describes the operational systems and tools that each group used in order to reach the agreement necessary to create its outputs and address the issue at hand.

The operational process itself has several components. Because every group at least implicitly seeks to identify and implement solutions to problems, decision-making processes are a critical part of group operation. Decision making, however, is only one of several operational components. Additionally, some groups have systems for addressing internal group conflict, pathways for interacting with other groups and organizations, processes for building and 
maintaining institutional memory, approaches for motivating participants, and systems for enabling both internal and external communication.

\section{Decision-Making Procedures}

The case studies exemplify a spectrum of decision-making procedures, ranging from consensusoriented deliberative approaches to a single point of unilateral control. This spectrum exists not just across case studies but within them, indicating that most groups utilize a range of procedures that are matched to the particular stage of the process and the context.

The strongest example of consensus-oriented decision making among the case studies is NETmundial. Prior to the meeting in Brazil, participants submitted public comments. An Executive Multistakeholder Committee (EMC) was responsible for synthesizing these comments into the draft outcome document. During the drafting process, the EMC required consensus on every single paragraph. Following public deliberation and input at the plenary sessions, the EMC further revised the document into the final outcome document. Although consensus was strictly adhered to, the decision-making process was limited to the relatively small EMC.

Other case studies describe groups that begin with consensus procedures but switch to other approaches as necessary. The EIDG and the TIIB are examples of this mixed use of procedure, using consensus on the less controversial matters and voting on the rest. In the case of the EIDG, this meant that individual working groups often reached consensus on the factual assessment portions of their reports. For the recommendations, however, there was a greater chance of disagreement, particularly on current political issues. These conflicts would be escalated out of the working group to the EIDG plenary, which would use majority voting to decide what text would be included in the working group report. At times, voting would proceed sentence-bysentence through the report. Similarly, the Creative Commons Corporation tries to achieve rough consensus within the community. Should that not work, however, the Corporation reserves the right to make decisions on its own.

\section{Conflict Resolution}

As described above, at the terminal point of making decisions about outcomes, groups can rely on the decision-making procedures described above to resolve conflicts. However, sometimes conflicts between stakeholders emerge at points in the process where there is no final decision to be made or where voting or other outcome-oriented responses would be inappropriate. One approach for helping to resolve these kinds of non-terminal conflicts is highlighted in the TIIB case, where the Board used a "cooling off" period to defuse tensions in conflicts where no solution was readily apparent. Such an approach does not in and of itself resolve the underlying conflict, but it provides both space and time so that a resolution might be easier to identify or more palatable to the parties.

\section{Interfaces and Coupling Management}

The groups we studied operate within a network of existing institutions. These groups often have formal and informal mechanisms for interacting with those existing institutions, particularly government bodies. The EIDG and TIIB both offer examples of formal interfaces between the groups and government. In both cases, the groups were designed to help improve legislative outcomes, so it was important to have established, formal pathways between the groups and the government. The primary interface mechanism in these cases was having governmental 
stakeholders play active roles within the groups, participating on equal footing with other stakeholders. Interestingly, neither group had a formal mechanism for turning proposals into legislation, but the active engagement of governmental actors at least created a pathway to ease the creation and introduction of such legislation.

In other cases the interfaces between groups and government are more informal. In the Swiss ComCom Roundtables, for example, active governmental involvement was limited to convening the Roundtables and the working groups. The primary governmental interface was the threat of future regulation in the event that stakeholders failed to reach a suitable agreement. In this case, the parties arguably participated within the Roundtable process out of a desire to avoid a more formal interface with government; the lack of a formal interface represented a form of interaction in and of itself, albeit a more passive one.

Government is not the only institution with which groups interact in the case studies. For example, the planning and operation of NETmundial required interfaces with the so-called I* organizations (e.g., ICANN, IETF, ISOC, IAB). Representatives of the I* organizations played a significant role within NETmundial, helping to shape the meeting and serving on several of the key committees. In several instances, platforms such as GitHub in the Bitcoin case or Adhocracy in the EIDG case have played crucial roles in sustaining interfaces. These platforms in turn enable interactions with existing communities of stakeholders or other interested parties.

\section{Knowledge and Memory Management}

The case studies evidence a spectrum of approaches to maintaining knowledge and building institutional memory. These approaches differ primarily in how easily each approach can be leveraged to affect the groups' future decisions. At one end of the spectrum, institutional memory is largely stored within the individual memories of staff. Creative Commons embodies this approach, where such human-centric institutional memory is supplemented by publiclyarchived e-mail lists and documentation of all important decisions. Such an approach makes leveraging this institutional knowledge more challenging because it relies upon the availability and memory of certain key individuals.

Relying instead on a more comprehensive, public, online database of material makes leveraging institutional knowledge easier. NETmundial is a good example of this approach. In that case all of the input documents were not only available online, but were accessible in formats that allowed third parties to analyze and assess contributions. The use of such a database lowers the cost of acting on institutional knowledge and reduces points of failure.

Still one step further is the use of technology to automatically log not just every discussion but the state associated with every decision, so that the group can easily undo bad decisions. This approach was taken by Bitcoin, which used GitHub for tracking both the debate related to decisions about the core Bitcoin code and the decisions themselves. Because GitHub is designed for code-based projects, it was a particularly well-suited tool for Bitcoin.

\section{Motivational Issues}

Successful operation of the groups described in the case studies required committed and motivated participation from the stakeholders and participants. In most of the cases the primary motivation was a shared desire to solve the problem that the group convened to address. However, the full scope of motivating factors is likely as diverse as the number of participants (if 
not more so, given that the individuals and organizations profiled in the case studies often held multiple explicit and implicit motivations). Often, these motivations may be layered and coexisting as the actors involved in a case may have primary, secondary, or tertiary motivations shaping their actions.

As an example, one layer of motivations revealed in the cases clustered around two dimensions: monetary and non-monetary. Some of the parties in the Swiss ComCom Roundtables, for example, recognized that it would be better if deployment of fiber optic cable to the home could be done faster, with less disruption, and avoid regulation. A faster, less disruptive, and less regulated deployment would make it more likely that the utilities and telecom providers would lower their initial costs and begin profiting from their investments sooner. Achieving these goals, however, would require cooperation, and the Roundtables provided that mechanism. In contrast, other motivations are non-monetary. For example, ComCom itself may have been motivated more by the opportunity to exert influence over an area outside its regulatory scope than any monetary incentives. Other forms of non-monetary motivations can be seen among Creative Commons participants, who were in part motivated by academic goals (i.e., extending the licensing standard to include science research formats) and personal goals (i.e., the opportunity to participate in a strong interconnected network of individuals and experts who share common values and interests).

\section{Communication}

Within the case studies we observe two forms of communication: internal communication among participants and external communication with the broader public. The case studies demonstrate that both are important and that both are challenging to do effectively.

Internal communication helps participants contribute more effectively to the outcome of a group. Unclear communications can lead to wasted or duplicated effort. NETmundial offers one example, where vague and inadequate communications regarding the roles of the various committees left some participants duplicating work or changing tasks at the last moment. Untimely communications can leave participants feeling underprepared, as was reportedly the case in some of the Swiss ComCom Roundtables.

External communication helps outside observers understand the process and outcome, ultimately helping build support for the work of the group and enhancing the perceived legitimacy of the outcome. When successful, communication (often enabled by Internet technologies) can even extend the breadth and depth of participation in the process itself, enabling far-flung interactions and creating a multidirectional communications stream. In the case of NETmundial, technology allowed the organizers to collect content submissions and comments on the draft outcome document. Platforms such as Adobe Connect enabled remote participation for both the planning meetings and the event itself. Another example is the Marco Civil's use of e-Democracia and Cultura Digital for enabling communication and online participation.

In some of the case studies, external communication and interactivity was challenging. In EIDG, for example, the Commission's online communications and participation tool was significantly delayed until the process was already well underway. Once it was made public, it was not fully integrated into Commission's working procedures. Although it generated 30,000 visitors and 3,250 active participants who performed 80,000 actions on the platform, the overall extent of participation did not meet the original expectations for the platform. Moreover, not all 
Commission members were equally willing to incorporate public feedback into their outputs, largely on account of the perceived increased effort this would take.

\section{Outcomes}

The tangible products (e.g., reports, proposals, agreements, guidelines, legislation, etc.) of a multistakeholder initiative are important, but our case studies show that participatory processes often create more ephemeral outcomes, too. Some outcomes are shared across all stakeholders, and some accrue more to individuals. Some outcomes are intended, and some are not. Some outcomes culminate the actions of the group, and some develop into separate ongoing actions. Ultimately, whether these outcomes represent "success" for the group depends on a variety of factors, tied to the purposes for which the group was originally formed and the legitimacy with which it operated.

\section{Outputs, Outcomes, and Impact}

Multistakeholder groups can yield many different kinds of outputs and outcomes, sometimes simultaneously. The case studies generally reflect coordinated efforts to address particular problems. Thus, a common output is a document that embodies the decision or identified solution. We see this in the Creative Commons case, where the outcome was version 4.0 of the licensing standard, and in the Aviation Slotting case, where the outcome was a set of guidelines for managing international slotting and busy airports.

Not all outputs are tangible, however. For instance, NETmundial, although it had a tangible output document, it also had an intangible output in the form of impact on other Internet governance fora, such as the UN's Internet Governance Forum (IGF). The IGF has largely eschewed concrete outcomes and instead focused on promoting dialogue between participants, largely out of the belief that achieving consensus on specific issues would be too difficult. However, in the wake of the success of NETmundial, several participants at IGF 2014 suggested that IGF could and should rethink its objectives vis-à-vis outcomes. This sense of empowerment that NETmundial helped to generate at other venues is itself an important outcome.

Although NETmundial had global impact, an outcome need not be so widespread to be successful. In fact, some outcomes are limited to a small group of people or may even be personal in nature. For example, the Marco Civil case study illustrates how the process of discussion and collaborative drafting assisted in the building of ongoing alliances. Those alliances became important for obtaining the eventual passage of the bill and monitoring its enforcement. Similarly, the Creative Commons case study shows how one outcome helped participants build a network of knowledgeable peers. In both cases, an outcome was the construction of relationships and alliances.

\section{2. $\quad$ Unintended Consequences}

In some cases the intended outcomes are not the only ones that occur. For example, in the Swiss ComCom Roundtables, the final four-fiber agreement ended up having significant unintended effects on the utility companies and the utilization of their fiber. Because of the utility companies' lack of experience as network managers and a competitive disadvantage against Swisscom, who could negotiate license agreements at a national instead of local level, the utility companies found themselves with under-utilized fiber. Similarly, the Aviation Slotting case study shows that the slot allocation standard has sometimes created unintended local problems 
related to antitrust and other regulatory concerns. In both cases, the unintended consequences seem to emerge from interactions between the group's decision and external factors (and a failure to properly anticipate such interactions). In the case of the Swiss ComCom Roundtables, the issues emerged when the four-fiber plan interacted with content licensing agreements. In the case of the Aviation Slotting guidelines, the issues emerged when the guidelines interacted with existing local institutional structures.

\section{Operational Continuity}

Once a group has identified and implemented a solution, the group does not necessarily need to continue operating. With the exception of EIDG, our case studies do not include any examples of organizations that completely disbanded after identifying solutions to the problems they were formed to address. This may be a result of the small sample size, but it may also reflect the fact that few solutions are self-executing. For example, in both the TIIB and the Marco Civil case studies, continued operation of the alliances and networks that formed throughout the processes was necessary in order to keep pushing for legislative progress and to monitor enforcement. In other cases, such as the Swiss ComCom Roundtables, active pressure and work was no longer needed, but the group was never formally disbanded, leaving open the possibility for it to be reconstituted if needed in the future., In other cases there is never a complete solution, and the outcome is ongoing management functions, such as with the INCB. And even with EIDG, although the commission was formally disbanded, its work lives on through pending legislative proposals and the formation of a permanent legislative committee on digital society within the parliament.

\section{Critical Success Factors}

Having described the common techniques, approaches, tools, and patterns observed within the case studies, we now turn toward critical success factors.

The governance bodies that have been objects of the case studies are almost all subject to specific scrutiny because they operate outside or on the fringes of common governance structures. To be deemed successful they are not only measured by the degree to which they have achieved their objectives and thus proved to have been "worth the effort." But they also must show that they have integrated safeguards into their structures and procedures that are at least functional equivalents of those present in the common governance environment in which they operate. These safeguards include ensuring inclusiveness, transparency, accountability and legitimacy, all of which go beyond mere effectiveness. In both the case of meeting objectives and including safeguards, the definition of "success" is inherently context-dependent, as what is successful for one group may not align with the objectives, inclusiveness, transparency, accountability, or legitimacy of another.

Although context dependent, the case studies help illuminate the interactions between a group's structural elements and the context in which it operates. While our case studies describe groups that were largely successful (as defined within their own context), the groups and activities represented within those case studies were not without fault. In almost all case studies, some techniques and tools worked better than others; and in some cases experiments and approaches simply failed.

\section{A. Inclusiveness}


One metric by which to evaluate governance groups is the degree to which they are inclusive of a diverse array of participants and stakeholders. That is not to say that more inclusiveness is necessarily better, but to instead consider the extent to which the structure and tools of the governance group enabled the greatest degree and diversity of participation within the constraints and needs of the group. There are many avenues for participation, each offering different kinds and depths of involvement. Perhaps the most obvious avenue is through formal inclusivenesshaving a seat at the table and being able to contribute to the discussion. Offering input, however, is different from decision making; another avenue of participation is through being able to vote on or otherwise affect the outcome or decisions of the group. Participation is sometimes mediated through other means, such as technological tools or through sub-entities like working groups. Regardless of the available avenues of participation, inclusiveness requires consideration of how well the stakeholders can actually make use of these options.

\section{Formal Inclusiveness: Identifying and Including Stakeholders}

One avenue through which a governance group can increase inclusivity is by formally inviting a larger array of individuals and organizations to participate in the discussion as stakeholders. As noted in Part II, all of the case studies describe groups that are multistakeholder. But the word "multistakeholder" does not convey the nuance of arrangements and the complexity of how these groups are crafted. For example, "multistakeholder" does not mean that every possible stakeholder is included. Although reasons for this varied, in all of the case studies, the conveners exercised some degree of control and discretion over the number and types of participants.

There are many reasons why conveners would want to exercise control over the selection of participating stakeholders. Perhaps the most common rationale for participant curation is to ensure that participants have a significant connection to the topic at hand. To some extent, this is obvious: a "stakeholder" is literally someone with a stake in the issue or outcome. However, we see from the case studies that determining the level of connection sufficient to generate a stake, and excluding the rest, often results in greater specificity in selection. For example, governance groups have enforced a nexus between topic and potential stakeholder by looking at the level of economic investment (Swiss ComCom Roundtables) or at technical expertise or involvement (IXPs). And in the Israel National Cyber Bureau case study, the nexus requirement was directly related to the sensitive nature of the national security topics.

Topical relevance is only one reason for curation that we observe in the case studies. Other rationales tend to be more practical in nature. For example, NETmundial faced space and funding limitations that required selecting from a larger pool of potential participants. In the case of the TIIB and the EIDG, the significant government involvement in those governance groups meant that participants were selected for their compatibility with the policymakers involved.

Even the most open of the governance groups still involve a basic level of participant curation. For example, the Bitcoin case study describes the process of revising Bitcoin's core codebase as a "meritocracy," where anyone can submit code revisions. However, only a handful of participants are core developers, and only a core developer can invite a participant to become elevated to the status of core developer. Thus, while basic participation was open to all, the overall organization was focused on a more select group.

Whatever the reasons for the curation of participants, these decisions are rarely static. Instead, the inclusiveness of governance groups often changes over time. For example, the Swiss 
ComCom Roundtables were formally limited to the CEOs of companies that had already invested in fiber optic cable deployment. This decision excluded some organizations, such as homeowners associations, that had a strong interest in the outcome. During the course of the Roundtables, ComCom reached out to these organizations and even invited some to make presentations to the Roundtables.

The dynamic nature of participant inclusion highlights an important observation from the case studies: the conveners of the observed governance groups often approach decisions about inclusiveness instrumentally. In other words, the conveners use different configurations of participants in order to achieve strategic aims. Interestingly, our case studies do not evidence many, if any, detrimental effects from this instrumental approach. This could be because a successful outcome helps erase any concerns about inclusiveness. However, the more likely reason is that achieving a successful outcome often requires the full buy-in and participation of critical stakeholders, so any instrumental approach to inclusiveness is bounded by the need to maintain the support of those stakeholders necessary for developing and implementing a solution. We see this in the CT-Spam case, where CGI.br required the full support of the ISPs, technical, and consumer communities in order to implement its desired solution.

\section{Voting, Consensus, and the Use of Decision-Making Processes}

Formal participation in a multistakeholder process is important for stakeholders because it offers the opportunity to shape the final outcome. Thus, equally important to stakeholders are the mechanics of decision making that determine that final outcome. The array of decision-making processes is described above in Part II. What we observe from the case studies and their deployment of processes is that consensus is generally preferred, but that governance groups will revert to voting where consensus cannot be reached and a solution can still be implemented over objections.

That consensus is generally preferred is not a surprise. For example, in both the Swiss ComCom Roundtables and the CT-Spam cases, the lack of regulatory authority to force implementation of the solution meant that a successful outcome required the voluntary cooperation of all stakeholders. Because of this, consensus was required to ensure that the stakeholders would buy into, and take ownership of, the solution.

Governance groups switch to voting only when a solution can be implemented over the objections of stakeholders. Voting as a decision-making process suggests that there is disagreement regarding the outcome. It is only a reliable option when full acceptance of an outcome is not necessary or when dissenters can be compelled to accept a resolution. This often occurs when there exists a recognized regulatory or legal authority to impose a solution over objections. For example, the EIDG could switch to voting for two reasons: (1) the Commission's outputs were reports and recommendations for parliament as opposed to items that the commission members could directly act on; and (2) to the extent that the German parliament wanted to act on recommendations of the EIDG, the parliament has the authority to enact laws without the full cooperation of all stakeholders. In that regard, the choice of decision-making process is closely related to the ability of the governance group to implement any given outcome. Switching to voting may come with the additional cost of changed participant behavior; the move from consensus may signal the acceptability of more adversarial processes and positions. In other words, a switch to voting when there is disagreement regarding the outcome may itself 
trigger greater disagreement. In the case of EIDG, the switch to voting pressured the participants to align with their political allegiances.

In some cases, governance groups use different decision-making processes at the same time in different parts of the same organization. For example, the Bitcoin case study describes several components: the Bitcoin code development on GitHub and the more formal Bitcoin Foundation. The development of Bitcoin code relies on GitHub forums to facilitate decision making. Through this medium, consensus is the simplest method for decision making, as voting would be challenging to implement in that environment. However, the Bitcoin Foundation, an organization that supports the development of Bitcoin, is a formal entity incorporated under the law of the District of Columbia, with formalized roles and legal obligations, and therefore a more formal role for voting and process. Although the level of interaction between the development of Bitcoin code and the Foundation is not entirely clear, we once again observe an increased significance of voting where it is supported by legal authority, even in an organization that generally uses consensus.

\section{Effect of Alternative Participatory Mechanisms}

Enabling in-person stakeholder participation is only one method of inclusion. The case studies demonstrate an array of alternative participatory mechanisms, some more effective than others. In several case studies, the use of mediating technologies was effective at expanding the array of participants to a wider community of stakeholders. For example, the Marco Civil case study describes how tools like eDemocracia allowed for collaborative drafting among members of the public.

That said, participatory technologies will not themselves automatically generate greater inclusiveness. Like any tool, participatory technologies must be used with intent and purpose. The remote hubs at NETmundial are an example where the organizers worked to ensure that individuals at the remote hubs would be able to participate actively and meaningfully in the discussion as though they were present in the room. Where online tools are not fully integrated into the workflow or embraced by the organizers, they were less effective. For example, in the EIDG case study, the delays in deploying the Adhocracy online platform meant that when it was ready, organizers had already developed their workflows. Participatory tools are not a panacea, but are instead a useful tool that can be deployed when their use is carefully considered and designed into the governance groups as a whole.

The case studies also contain examples of alternative participatory mechanisms that are not technology-based. The primary example from the case study is the use of working groups. Generally these working groups are used as a way to reduce friction in the decision-making process. For example, in the Swiss ComCom Roundtables, a working group was used to simplify decision making regarding less controversial technical decisions. Similarly, TIIB used a working group to bypass the more constraining membership requirements of the Board in order to include a broader array of viewpoints and perspectives. In contrast, the EIDG used working groups not as an alternative means of participation, but as the primary operational structure for the governance group.

\section{Resource Asymmetries}

Formal participatory roles or tools can increase inclusiveness only if participants and stakeholders have the ability and resources to take advantage of those opportunities for 
participation. Financial resource limitations constrained who was able to afford to attend the NETmundial meeting. Although funding was eventually secured to help support the attendance of those who could not afford to attend, by then it was too late for many to change plans. Similarly, both time and finance constraints affected who could participate in the EIDG working groups; participants who had funding to support their attendance and provide staff support could contribute more actively, while those who were simply volunteering on top of their normal jobs were more limited. And in the ComCom Roundtables, knowledge asymmetries about the telecommunications industry placed the utility companies at a disadvantage in negotiations. In some cases the constraints are less explicit. For example, in the Bitcoin and IXP case studies, technical expertise was an implicit limitation on participation.

It is certainly possible to overcome these limitations and asymmetries, but it generally requires both time and money. For example, additional funding eventually provided for expanded participation at NETmundial. More challenging is addressing knowledge asymmetries and engaging in capacity development, as this can take years, depending upon the starting circumstances. The WVBB case study mentions how the process of building capacity in water resources management involved developing a core of teachers who could then work over the course of years train the necessary team of skilled professionals who could implement and manage the system. Addressing these asymmetries in governance groups is important for increasing inclusiveness, but the difficulty of doing so should not be underestimated.

\section{B. Transparency}

In the same way that the tools of inclusiveness are often deployed in instrumental ways to achieve strategic objectives, transparency is also an instrumental tool within the case studies. The case studies show that often the level of transparency that a governance group uses can be moderated to the specific needs and circumstances that the group faces over its lifecycle.

Within the case studies, transparency is the effort at communicating with both the stakeholders and the public more broadly with respect to the decision-making process of the group. The approaches to transparency within the case studies spanned a wide spectrum, from very limited openness to fairly radical depths of transparency. The Swiss ComCom Roundtables represented a more limited approach to transparency: meetings were closed, and the parties agreed that public communications about the Roundtables would come from ComCom and not from individual stakeholders. Thus, the public record of the Roundtables is dominated by a single source of information mediated through a single entity. NETmundial and Marco Civil, by contrast, represent more transparent approaches. For example, in NETmundial all of the input statements were made publicly available online where they were searchable and open for research and analysis. Similarly, in Marco Civil, organizers required stakeholders to publish their comments online in a way that clearly identified the stakeholder and their position, allowing an opportunity for feedback and criticism.

Although the case studies used different approaches to, and levels of, transparency, in many cases the driving motivation was the same: what approach would best support the identification and implementation of a solution? In Marco Civil, for instance, the organizers believed that given the complexity of the negotiations, transparency would be necessary to help lower the barriers to reaching an agreement. In the ComCom Roundtables, ComCom believed that open meetings would make it more difficult for the stakeholders to compromise in the discussions. 
This fear may not have been unfounded, as the EIDG case study notes that the use of public meetings often led to political posturing on the part of the stakeholders, at the expense of engaging more deeply in the substance of the debate.

Accordingly, it seems that in some circumstances transparency can aid the deliberative process, while in other circumstances it may be a hindrance. The case studies suggest that one explanation of this divergence may be the background and previous experiences of the stakeholders and participants. In the context of the Internet space more broadly, transparency is a more established norm, and stakeholders who are more familiar with that environment may be more inclined to believe that transparency assists in the deliberative process. For example, the Bitcoin case study featured a community of highly technologically adept stakeholders predisposed to be more comfortable with having debates about the code in public forums. In contrast, EIDG and ComCom involved more politicians and established telecom incumbents, respectively, who may not be as steeped in the norms of transparency. This suggests that the level of transparency may be a reflection of the norms of the community instead of something that in the abstract aids or hinders deliberation; it may be self-fulfilling that a community that is primed to be suspect of transparency will find it to be hindrance to a successful deliberation.

Different contextual environments may also influence how decisions about transparency are perceived more broadly. In general, regardless of the level of transparency that a governance group used, there did not appear to be a significant negative impact on the outcome or on the perception of the group as a whole. This is not to suggest that all approaches were without fault. As noted previously, the transparency of the EIDG workshops was criticized for producing an overly political environment. In the ComCom Roundtables, some participants criticized the lack of transparency as a way for ComCom to control the messaging and create an appearance of greater authority. And even within the context of a fairly transparent approach, participants at NETmundial criticized the transparency of the drafting process and the selection process for committee representation. However, in no instances were transparency decisions (or lack thereof) viewed as fatal to the work of the group. This suggests that at the very least the level of transparency within the case studies was generally well-matched to the expectations of the community affected by the work of the governance groups.

\section{Accountability}

Although accountability has not emerged as a primary issue in these case studies, we do see various "traces" of it. While the analysis of the case studies does not point to a singular notion of accountability, we can identify a number of tools through which accountability is imposed or procedural limitations restraining the unbridled operation of a governance group. These limitations can take many forms, including voting for (or removing) key decision makers, leaving the governance group for alternatives, subjecting group decisions to oversight panels, offering opportunities for public deliberation and protest, and requiring various forms of transparency, among others.

One of the most common forms of accountability that we see in the case studies is the threat of defection to alternate forums. For example, the Bitcoin case study describes how the developers of the Bitcoin core code were inherently conservative in their actions due to the paramount concern of maintaining a unified Bitcoin architecture. The key developers explicitly described how the relatively low transaction costs of forking the code and creating a competing system 
limited their actions. Similarly (although less explicitly), the Creative Commons and NETmundial case studies describe situations where the outcomes (CC licenses and the NETmundial principles, respectively) were not self-executing and required continuous reaffirmation, meaning that people must continually opt in to their use. For instance, publishers of scientific data must continually opt in to the use of the Creative Commons 4.0 license for each new data set, meaning that abandoning Creative Commons licenses is always an option.

In addition to the threat of defection, we also see several other forms of accountability in the case studies. For instance, the use of public deliberation and criticism is described above and below. The case studies are mixed with respect to the effectiveness of the tools used to enable enhanced public deliberation. As a lever of accountability, public deliberation can be no more effective than the tools that enable that participation in the first place. In other words, if public participation is not fully realized or implemented within the governance group, it is unlikely to serve as a significant source of accountability. Transparency is yet another form of accountability that we observe in the case studies, and which is described more fully above.

To a lesser extent we also observe the use of both voting and oversight authority as levers of accountability within the case studies. For example, in EIDG, half of the Commission members were elected members of the German parliament. Although the public support of those elected members was likely tied to many more issues than just their activities on the Commission, it remained possible for those members to be removed from office. Additionally, both the EIDG and Marco Civil case studies offer examples of oversight authority. Both cases resulted in legislative proposals that required the action of a separate legislative body in order to become law. This separation between drafting and enactment provided accountability to the governance groups by limiting their ability to unilaterally implement their proposals.

In looking more broadly at the use of levers of accountability within the case studies, we observe a relationship between the methods of accountability used and the other defining elements that we explore elsewhere in this paper. For example, where formal regulatory or legal authority underlies the operation of the governance group, the use of voting or oversight committees appears to fit quite naturally as an accountability tool (e.g., EIDG and Marco Civil). However, in the absence of regulatory authority, it is often easier for stakeholders to leave the governance group and create alternatives, meaning that defection and transparency might be more effective levers of accountability (e.g., Swiss ComCom, Bitcoin, and Creative Commons). Accordingly, the use of accountability tools should not be viewed as an independent matter, but should instead be viewed in the context of the overall operation and structure of the governance group as a whole.

\section{Legitimacy}

Perceptions of the legitimacy of governance groups also proved an important contextual factor. As highlighted above, many of the governance groups we examined relied on multiple sources of legitimacy, which frequently included a combination of traditional sources, such as government, as well as non-traditional sources such as expertise or broad participation. In Section III above, we reviewed the range of sources of legitimacy in the case studies; here we reexamine legitimacy's role as a factor in the success of the governance groups.

The case studies highlight the variety of ways in which legitimacy can shape a governance group's success. In some cases, input legitimacy was a critical source of legitimacy. For 
example, in the wake of the Snowden revelations, most stakeholders in the Internet governance community agreed upon the need to stage a public discussion about Internet governance. However, the appropriate process and platform for holding that debate was highly contested, making the legitimacy of the NETmundial process a critical component.

In other cases, output legitimacy is more central to the success of the group. For example, in the Swiss ComCom Roundtables, the outcome was an agreement that improved the deployment of fiber optic cable across Switzerland. Despite concerns over inclusion of stakeholders within the process, the Roundtables have been perceived largely as legitimate due to the success of the outcome.

Interestingly, in some case cases legitimacy was itself a key deliverable for the group. At NETmundial, it was important to demonstrate that multistakeholder processes could be used to create principles for governance, making the legitimacy of the output and the process a goal in and of itself. In contrast, legitimacy was not an end in and of itself for the CT-Spam governance group. This was largely because once implemented, the Port 25/TCP change did not require continued buy-in and reaffirmation from stakeholders, and had relatively little capacity to cause public harm and great potential to enhance the public good. Although legitimacy in the process was necessary to obtain buy-in from the key stakeholders, it was not necessary as an outcome.

In addition to highlighting the ways in which legitimacy may be foregrounded at different stages of the governance process, the case studies also indicate that the centrality of legitimacy is linked to the alignment between the interests and preferences of stakeholders. In domains that are more politicized or where the positions of stakeholders are more divergent, legitimacy is more frequently called into question. In these instances, "stronger" forms of legitimacy become necessary for the perceived success of the process.

\section{E. Effectiveness}

Judging the overall effectiveness of governance groups is challenging because effectiveness must be assessed with respect to the groups' unique context, objectives, and needs. The case studies covered a wide-range of outcomes, spanning convening to legislation. But whether those outcomes were effective depends on how well the governance group met initial expectations and achieved its goals. There is no one definition of effectiveness that can be applied across all the case studies. Rather, success is highly context-dependent, and should be measured at two levels: internally and externally. Internal definitions of success are generally more process-oriented, focused on whether the multistakeholder group operates as effectively as possible given its context. External definitions of success are generally objective-oriented, focused on whether or not a stated goal was achieved.

The very process of clearly defining a mission and outcomes can positively contribute to the overall effectiveness of a governance group. Vagueness around a group's objectives can obfuscate the process of achieving consensus, and increases the vulnerability of a group to be influenced by political pressure. For example, a number of EIDG members expressed regret regarding what they perceived to be the overly broad focus of the Commission. They felt that if the group had a more clear and narrowly defined objective, the Commission might have achieved more significant results. Similarly, the mission can be more clearly defined by separating more technical decisions from policy ones. This division can improve effectiveness by freeing the stakeholders to reach consensus on less controversial decisions without resolving all of the 
political concerns that may have motivated the formation of the governance group in the first place.

The efficiency of the governance group has an impact on its effectiveness, but whether a governance group operates with sufficient speed appears to be a relative concept. In some cases, multistakeholder processes may have provided efficiencies for policymakers by filling in where regulations were not able to keep up with technological development. For example, the Swiss ComCom Roundtables benefitted from the relative efficiency of governance group as opposed to the more lengthy process of amending the telecommunications law to address fiber optic cable. In other cases, multistakeholder processes can be slow and arduous due to the need to derive input and achieve consensus from stakeholders who may or may not easily reach agreement. Though this can create frustrations among stakeholders, the pace of progress should be carefully calibrated with other objectives. For example, the authors of the CT-Spam case note that the technological solution to the spam problem was implemented in many countries around the same time, but those countries with greater centralized regulatory authority were able to implement the technical change far faster than Brazil. However, the multistakeholder group recognized that in Brazil a top-down process, while speedier, would have proved ineffective in the long run because the participants would not have fully supported or implemented the solution. In fact, the highspeed process at NETmundial may have negatively influenced the full inclusion of views by leaving insufficient time for stakeholders to achieve internal consensus. Thus, some level of efficiency may need to be sacrificed for the sake of long-term effectiveness.

Operating at multiple levels of scale can also contribute to the effectiveness of groups. For example, Swiss ComCom and the EIDG used smaller working groups to develop concrete solutions with more technical knowledge before passing these solutions up to broader higherlevel plenary groups. Expertise was closely tied to questions of scale: in some instances, such as the EIDG, groups found it beneficial to develop solutions at a technical level before issues could become politicized, while in the CT-Spam group actors were hesitant to implement technical solutions without having input from a larger array legal and policy advisors. In still other cases, such as the TIIB and the INCB groups, efficiencies were achieved at the expense of full participation by limiting the number of stakeholders who could participate.

\section{Summary of Observations}

The analysis of the case studies from a variety of governance contexts reveals a complex and nuanced picture. We distill those findings below in three categories: (1) their purpose and context; (2) their formation and operation; and (3) their critical factors for success. With respect to the purpose and context for and in which multistakeholder groups are formed and operated, we identify the following key findings:

- Multistakeholder groups can serve a broad range of purposes, ranging from open-ended missions (e.g., gaining a deeper understanding of complex issues by incorporating views of multiple actors) to issue-specific tasks (e.g., execution of a particular policy). The primary mission is often supplemented by secondary purposes such as network building or formation of communities of knowledge and practice.

- Contextual factors, including culture, are key variables that have the potential to shape almost all dimensions of multistakeholder groups, from formation to dissolution. In 
particular, pre-existing relationships, cultures of problem-solving, and the availability of resources need be taken into account.

- Finding alternative sources of legitimacy is critical for groups that cannot build upon traditional sources.

We observe significant variations in the case studies when it comes to the formation and operation of governance groups. In many cases these differences are shaped by the unique purposes and contexts of the group. The key takeaways from the analysis include the following:

- There is no standard way to form multistakeholder groups. While in all cases the composition of the group is a critical element of both the formation and the ultimate outcome of the process, inclusion criteria as well as internal structures and architectures vary greatly from one case study to the other.

- The multistakeholder groups analyzed in this synthesis share a common collaborative mode and bring together distributed resources. The operational process itself, including systems and tools, is managed very differently from group-to-group, however. For instance, decision making and conflict resolution procedures range from consensusdriven to hierarchical, with all permutations in between. Similarly, interfaces with other institutions, communication strategies, and mechanisms of knowledge management range from formal to informal.

- With respect to outputs and outcomes, the case studies indicate the range of what multistakeholder groups can contribute to the world, including tangible outputs such as documents, specifications, standards or code and intangible outputs such as endorsement or empowerment. Not all of these outputs are intended; multistakeholder groups might also lead to unintended consequences, including complicated interactions with existing laws and regulations (e.g., antitrust issues).

In addition to (and interacting with) these structural elements, the analysis of the case studies has led to a series of observations regarding the critical factors for success of multistakeholder groups. These takeaways are:

- Inclusiveness (including mechanisms for participation) and transparency are critical factors to be managed and adjusted throughout the lifecycle of governance groups. The case studies revealed different formal and informal mechanisms of inclusion, as well as mechanisms of participation - such as consensus or voting. Similarly, transparency as an effort to communicate both within the group and with those outside is also an instrumental tool. Importantly, transparency and inclusiveness are dynamic characteristics and need to managed throughout the entire process, from formation and operation to the afterlife of a multistakeholder group.

- Accountability and legitimacy of governance groups are highly reflective of and dependent on contextual factors. The analysis of the case studies does not lead to a coherent concept of accountability, but highlights various elements of accountability, including direct approaches such as voting, and indirect mechanisms such as "defection" and opportunities for public criticism. These elements of accountability interact with other factors for success, such as transparency, and they need to be considered in the context of the overall operation and structure of the governance group. The case studies also highlight different kinds of legitimacy, ranging from input to output legitimacy, and 
demonstrate that governance groups might rely on overlapping sources of legitimacy at any given moment.

- The case studies suggest that effectiveness is defined with the context of the individual governance groups. In process terms, effectiveness describes the ability to operate within particular time constraints, but also manage pace over longer periods of time, and the ability to deal with multiple levels of scale, all of which might change over the life of a multistakeholder group.

\section{Conclusions}

As noted in the introduction, this document provides a high-level synthesis of a case study series that seeks to deepen our understanding of the formation, operation, and effectiveness of governance groups. The goal of this work, taken as a whole, is to inform the evolution of and current debate around the Internet governance ecosystem in the light of the NETmundial Principles and Roadmap, as well as the discussions at the Internet Governance Forum (IGF), the NETmundial Initiative, as well other forums, panels, and committees.

Using the final report of the Panel on Global Internet Cooperation and Governance and Governance Mechanisms as a conceptual starting point, the case studies were selected to ensure diversity of cases along several axes emphasized in the Panel's report. In particular, the participants in this collaborative research effort wanted to ensure that the case studies collectively inform us about: (1) how governance groups best match challenges with the organizations, experts, networks, and governing bodies/entities best able to help develop legitimate, effective, and efficient solutions; (2) how to structure the "flow of information and knowledge" necessary for successful governance; and (3) how different governance groups deal with coordination between regional and global (or national and regional) governance networks in order to avoid conflicting directives.

As to be expected, the real-world case studies examined in the context of this research effort only partially mapped onto the conceptual model outlined in the Panel's report. Specifically, the analysis and discussion of the case studies has led to a more diverse and nuanced set of observations regarding the structure and operation of such groups, as well as the factors that are important for their successful operation. Indeed, the analysis of a diverse set of real-world case studies suggests a far richer set of options than we originally anticipated in the formation and operation of governance groups. From the diverse cases we have studied, no clear dependencies or hierarchies have emerged between the different approaches available to define and manage the purpose, structure, process, or performance of governance groups.

At the most fundamental level, the review of the case studies suggests that governance groups work best when they remain sensitive throughout their entire lifecycle to the surrounding (and typically dynamic) contextual and cultural conditions, the availability of support systems, and the opportunities for and trade-offs related to inclusion, transparency, and accountability. The groups that are most sensitive to these factors and how they change over time are best positioned to be flexible and adaptive to new circumstances and needs. At the same time, the detailed analysis of various structural elements and mechanisms makes clear that there is no single model or way to run governance groups that would be of strong prescriptive value across contexts. Rather, our study sheds light on the various "tools" available in the toolbox when it comes to formation, operation, and outcomes of multistakeholder governance groups, and this synthesis strives to 
highlight the interplay between those elements. Similarly, the analysis of a series of critical factors - including inclusiveness, transparency, accountability, legitimacy, and effectivenessdoes not suggest a single approach that promises success across or even within governance contexts. While we cannot offer clear-cut "templates" or "model groups" based on our analysis, we instead offer observations and considerations with respect to a broad range of approaches, mechanisms, and tools and discuss these findings in the context of real-world situations.

What then is the secret to a governance group's success if it is not the application of a specific model or tool? Our case studies indicate that the most robust, effective, and legitimate groups are those that demonstrate sensitivity to the cultural environment in which they operate. In other words, it is attentiveness to both the "multi" and the "stakes" of "multistakeholder"-creatively and dynamically harnessing the multiplicity of forces and motivations into a collaborative body. This places a premium on leadership that can not only guide the group toward the its purpose, but can simultaneously and continuously screen the environment for enhanced sources of legitimacy, stabilizing relationships, and enabling partnerships.

Such conclusions are of course limited by our research intentions and the samples chosen. Despite those constraints, the case studies help us understand that despite challenges to their creation and operation as well as often-difficult regulatory environments, there remains a vibrant, open, and complex space for multistakeholder groups and their successful operations. Indeed, it is sometimes in the most challenging environments where formal regulation cannot or does not exist that the benefits of multistakeholder governance groups can be most impactful. We hope that the lessons from the case studies can help the conveners and leaders of future governance groups better understand and dynamically deploy the full range of tools and mechanisms available to them.

\footnotetext{
${ }^{1}$ The synthesis and each case study can be downloaded at http://ssrn.com/abstract=2549270.

${ }^{2}$ Panel on Global Internet Cooperation and Governance Mechanisms, Panel Report, http://internetgovernancepanel.org/panel-report.

${ }^{3}$ The process is documented here: http://networkofcenters.net/events.

${ }^{4}$ The notes from the meeting are available here: http://networkofcenters.net/event/evolution-internet-governanceecosystem.

${ }^{5}$ Minu Hemmati,. Multistakeholder Processes for Governance and Sustainability: Beyond Deadlock and Conflict (Sterling, VA: Earthscan Publications, 2002).

${ }^{6}$ Thomas Hale and David Held. The Handbook of Transnational Governance: Institutions and Innovations. (Cambridge: Polity Books, 2011).

${ }^{7}$ Markus Kummer, "Multistakeholder Cooperation: Reflections on the Emergence of a New Phraseology in International Cooperation” The Internet Society, 2013, http://www.internetsociety.org/blog/2013/05/multistakeholder-cooperation-reflections-emergence-new-phraseologyinternational.

${ }^{8}$ For examples, see:

Multistakeholder governance: Laura DeNardis and Mark Raymond, "Thinking Clearly About Multistakeholder Internet Governance” (November 14, 2013), http://ssrn.com/abstract=2354377; Minu Hemmati, Multistakeholder Processes for Governance and Sustainability: Beyond Deadlock and Conflict (Sterling, VA: Earthscan Publications, 2002); Carlos Afonso, Virgilio Almeida, and Demi Getschko, "The Origin and Evolution of Multistakeholder Models," IEEE Internet Computing 19, 1 (January 2015), 74-79.
} 
Decentralized governance: G. Shabbir Cheema and Dennis A. Rondinelli "From Government Decentralization to Decentralized Governance" in G. Shabbir Cheema and Dennis A. Rondinelli, eds., Decentralizing Governance: Emerging Concepts and Practices (Brookings Institution Press: Washington, DC., 2007).

Distributed governance: Frederick M. Abbott, "Distributed Governance at the WTO-WIPO: An Evolving Model for Open-Architecture Integrated Governance," Journal of International Economic Law vol. 3(1) (2000): 63-81; Jon Marshall, "Negri, Hardt, distributed governance and open source software," PORTAL Journal of Multidisciplinary International Studies vol. 3, No. 1 (January 2006), http://epress.lib.uts.edu.au/journals/index.php/portal/article/viewFile/122/84; Paquet, Gilles, "The New Governance, Subsidiarity and the Strategic State" OECD Forum for Future Conference, Governance in the 21st Century, (Spring, 2000), http://www.gouvernance.ca/publications/00-60.pdf.

Collaborative governance: Chris Ansell, and Alison Gash, "Collaborative Governance in Theory and Practice," Journal of Public Administration Research and Theory vol. 18(4) (2008): 543-571; John Donahue, "On Collaborative Governance," Corporate Social Responsibility Initiative Working Papers, No. 2 (March 2004).

${ }^{9}$ See Roxana Radu, Jean-Marie Chenou, and Rolf H. Weber, eds., The Evolution of Global Internet Governance (Zurich: Schulthess 2013) (including several articles describing different approaches to and types of multistakeholder governance).

${ }^{10}$ For the full version of the Aviation Slotting case study, see Appendix A. The case study is also available for comment at https://publixphere.net/i/noc/page/IG_Case Study Aviation_Slotting.

${ }^{11}$ For the full version of the Bitcoin case study, see Appendix B. The case study is also available for comment at https://publixphere.net/i/noc/page/IG_Case_Study_Bitcoin_and_Autonomous_Systems.

${ }^{12}$ For the full version of the Creative Commons case study, see Appendix C. The case study is also available for comment at https://publixphere.net/i/noc/page/IG_Case_Study_Creative_Commons.

${ }^{13}$ For the full version of the CT-Spam case study, see Appendix D. The case study is also available for comment at https://publixphere.net/i/noc/page/IG_Case_Study_Fighting Spam_the_Multistakeholder_Way.

${ }^{14}$ For the full version of the EIDG case study, see Appendix E. The case study is also available for comment at https://publixphere.net/i/noc/page/IG_Case_Study_EnqueteKommission_Internet_und_digitale_Gesellschaft.

${ }^{15}$ For the full version of the White Volta case study, see Appendix F. The case study is also available for comment at https://publixphere.net/i/noc/page/IG_Case_Study Water Management_in_Northern_Ghana.

${ }^{16}$ For the full version of the IXP case study, see Appendix G. The case study is also available for comment at https://publixphere.net/i/noc/page/IG_Case_Study_Multistakeholder_Governance_and_Nodal_Authority_Understan ding_Internet_Exchange_Points.

${ }^{17}$ For the full version of the INCB case study, see Appendix H. The case study is also available for comment at https://publixphere.net/i/noc/page/IG_Case_Study_Towards_a_Cyber_Security_Policy_Model_Israel_National_Cyb er_Bureau_INCB.

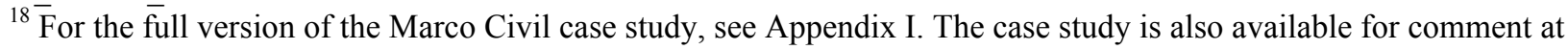
https://publixphere.net/i/noc/page/IG_Case_Study_A_Bill_of_Rights_for_the_Brazilian_Internet.

${ }^{19}$ For the full version of the NETmundial case study, see Appendix J. The case study is also available for comment at https://publixphere.net/i/noc/page/IG_Case_Study_NETMundial.

${ }^{20}$ For the full version of the Swiss ComCom case study, see Appendix K. The case study is also available for comment at https://publixphere.net/i/noc/page/IG_Case_Study_Swiss_ComCom_FTTH_Roundtable

${ }^{21}$ For the full version of the TIIB case study, see $\overline{\text { Appendix L }}$ The case study is also available for comment at https://publixphere.net/i/noc/page/IG_Case_Study_Turkish_Internet_Improvement_Board. 


\section{Appendices A-L: Case Studies}




\section{Appendix A: Aviation - The Worldwide Slot Guidelines}




\title{
NoC Internet Governance Case Studies Series: Aviation - The Worldwide Slot Guidelines
}

\author{
Giovanni Sartor and Hanna Schebesta \\ European University Institute
}

\section{Editorial Note: Context, Character, and Purpose of the Case Study}

This case study is part of a globally coordinated, independent academic research pilot project by the Global Network of Interdisciplinary Internet \& Society Research Centers (NoC). Facilitated by the Berkman Center for Internet \& Society at Harvard University, this study examines existing multistakeholder governance groups with the goal of informing the future evolution of the Internet governance ecosystem. Building upon the NETmundial Principles and Roadmap, it contributes to current policy debates at the international level, including the Internet Governance Forum, the NETmundial Initiative, and other organizations and efforts.

Internet governance is an increasingly complex concept that operates at multiple levels and in different dimensions, making it necessary to have a better understanding of both how multistakeholder governance groups operate and how they best achieve their goals. With this need in mind, at a point where the future of Internet governance is being re-envisioned, colleagues from several NoC institutions around the world have written twelve case studies examining a geographically and topically diverse set of local, national, and international governance models, components, and mechanisms from within and outside of the sphere of Internet governance. Key findings from these cases are summarized in a synthesis paper, which aims to deepen our understanding of the formation, operation, and critical success factors of governance groups and even challenge conventional thinking.

The research, based on twelve case studies, suggests that there is no single best-fit model for multistakeholder governance groups that can be applied in all situations. Rather, it reveals a range of approaches, mechanisms, and tools available for both the formation and operation of such groups. The analysis demonstrates that the success of governance groups depends to a large degree on the careful selection, deployment, and management of suitable instruments from this "toolbox." As governance groups pass through different phases of operation, conveners and facilitators must remain alert to changes in circumstances that necessitate adjustments to the approaches, mechanisms, and tools that they deploy in order to address evolving challenges from inside and outside. This case study provides insights into how those instruments can be deployed and adjusted over time within such groups, and highlights how their interactions with important contextual factors may be successfully managed within given resource restraints.

The research effort is grounded in a diversity of global perspectives and collaborative research techniques. Adhering to objective and independent academic standards, it aspires to be useful, actionable, and timely for policymakers and stakeholders. More broadly, the Network of Centers seeks to contribute to a more generalized vision and longer-term strategy for academia regarding its roles in research, facilitation and convening, and education in and communication about the Internet age.

For additional information on the initiative, please contact Urs Gasser, Berkman Center for Internet \& Society, at ugasser@cyber.law.harvard.edu. 


\begin{abstract}
This case study explores the Worldwide Slot Guidelines (WSG), which represent a set of agreements made under the auspices of the International Air Transport Association (IATA) in order to allocate airport capacity. The right to use airport capacity for the purpose of takeoff and landing operations at airports is commonly called slots, with each airport having a finite amount of airport capacity. Thus, the slot allocation process is one of resource management: the WSG were developed as a global industry standard to address a key constraint in the aviation domain. The actual process of slot allocation is complex and involves organizations and stakeholders at multiple levels, all falling under the umbrella of IATA, a trade association representing 240 airlines. It designates particularly congested airports, creates roles of airport coordinators, and establishes management principles for slot allocation. Because they manage a valuable economic resource, the transparency and independence of coordinators is of particular concern: this has resulted in the institutionalization of a negotiating process to ensure accountability among key stakeholders. Through an examination of the history and key points of change in the process, the WSG case presents an example of private sector coordinated resource management.
\end{abstract}




\section{Table of Contents}

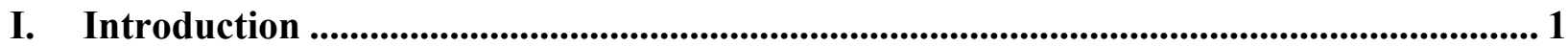

A. The Aviation Domain ......................................................................................................................... 1

B. The Problem: Slot Allocation ................................................................................................................. 1

II. Governance Through the WSG..................................................................................... 3

A. Mission and Function.......................................................................................................................... 3

B. The Functional Solution to Overcome Infrastructure Capacity Overloads ................................. 4

C. The Allocation Process........................................................................................................................... 6

D. Enablers ........................................................................................................................................ 7

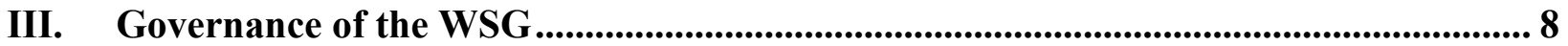

A. IATA/Slot Policy Working Group.................................................................................................... 8

B. World Wide Airport Coordinators Group ........................................................................................... 8

C. The Joint Slot Advisory Group.................................................................................................................... 9

D. Issue Identification and Change …………………………........................................................................ 9

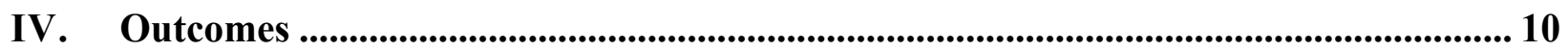

A. Evaluation of the WSG Operation ................................................................................................... 10

B. Stakeholder Views............................................................................................................................... 11

C. Cross-Sphere Synchronization....................................................................................................................... 12

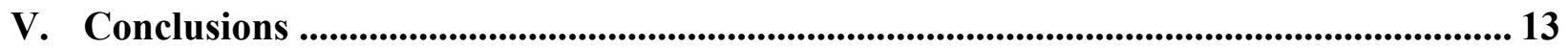




\section{Introduction}

The following case study discusses the Worldwide Slot Guidelines (WSG), an industry standard for airport slot management operated under the auspices of the International Air Transport Association (IATA). The focus of the study is on multilevel governance. It describes first the basic governance mechanisms through the WSG and governance of the WSG, followed by a critical discussion of the outcomes.

\section{A. The Aviation Domain}

Aviation is a truly global domain and a modern necessity, with connections that traverse local, national and international territorial boundaries and governance spheres. It is also a domain in which the optimization of the system's functions is of the highest priority; the need for functionality creates pressure for coordination at the global level. It is also a domain in which technological advancement imposes important constraints on the system functioning. There are strong requirements for uniformity or interoperability when it comes to innovation.

The aviation domain is marked by sovereign state responsibility and characterized by a territorial approach. The provision of air navigation services is assigned to entities by the state (designated air navigation service providers). This is still the case even though there are efforts to move away from the state-based territorial governance system, towards an approach focused on sectors involving multiple states (e.g., the Single European Sky) or in the competitive provision of noncritical services. ${ }^{1}$ Infrastructure capacity places very rigid constraints on the aviation domain. Opening new airports or creating new slots is highly cost intensive. ${ }^{2}$ Traffic patterns and demand for slots are mapped through long-term strategic planning for flight routes. Lastly, aviation is a high-risk domain, meaning that system failures result in catastrophic events and it is thus an area of high-risk regulation.

\section{B. The Problem: Slot Allocation}

Aircraft routes are so-called "highways of the sky," where aircrafts remain under direct air traffic control provided by the responsible air navigation service provider. Changes from one airway to another are carried out at waypoints. Take-off and landing operations require the use of an airport's terminal, runway, air traffic control, and other infrastructure and ground services, all of which comprise an airport's capacity. The right to use such airport capacity at a given time is commonly called a "slot."3

\footnotetext{
${ }^{1}$ See, e.g., European Commission, "Single European Sky II,"

http://ec.europa.eu/transport/modes/air/single_european_sky/ses_2_en.htm.

${ }^{2}$ According to an Oxera Consulting study prepared for the UK Parliament's Transport Committee in 2013, the indicative range of costs for a new airport is between $£ 10-15$ billion, depending on the number of runways and location. See Oxera Consulting Ltd., "Would a new hub airport be commercially viable?" (January 25, 2013), http://www.parliament.uk/documents/commons-committees/transport/Would-new-hub-airport-be-commerciallyviable.pdf.

${ }^{3}$ In the US the definition of a slot is a reservation for a takeoff or landing, not associated to further infrastructure apart from the runway; in the EU the reference is to a range of airport infrastructures and a slot is confined to the "coordinated airports" (i.e., Level 3 WSG congested airports). See David Gillen, "Airport Slots: A Primer" in: Achim I. Czerny, Peter Forsyth, David Gillen, and Hans Martin Niemeier, eds., Airport Slots (Hampshire: Ashgate Publishing 2008), p. 41.
} 
Airport slot capacity has emerged as one of the most important constraints of the aviation domain. ${ }^{4}$ The overall increase in demand is coupled with a limited increase of capacity. For example, in summer 2000 at Heathrow Airport there was a demand for 335,578 slots but only 283,681 were allocated. While it is acknowledged ${ }^{5}$ that slot allocation is not a solution to the underlying problem - infrastructure shortcomings - the scheduling of slots is one way to mitigate the capacity constraints. As such, aviation slotting is a way of regulating the access to a network by allocating access rights to infrastructure use.

Today, airport slot allocation is widely based on the WSG standard, which provides a global standardized process for airport slot management. The standard developed gradually over time out of practices occurring under the auspices of the IATA, the global airlines' umbrella trade organisation. This started in 1947, at a time when airlines were conducting bilateral negotiations in order to adjust their schedules. The 1960s saw a greater congestion at important airports, which resulted in a greater need for coordination between airlines and airports. Finally, the IATA codified this practice in the first Worldwide Slot Guidelines in 1976 according to the "consensus" of the community and has been maintaining the WSG ever since. The WSG are conceived as a "living document" that is adapted over time to meet operational challenges and the changing needs of the community. ${ }^{6}$

Under the WSG, management of airport slots is only necessary in the most congested airports. Accordingly, airports are classified as Level 1 "non-coordinated," Level 2 "schedule facilitated," or Level 3 "coordinated" airports. Worldwide, around 283 airports are Level 2 and 3 and subject to airport facilitation and coordination, respectively. ${ }^{7}$ Of these, around 174 are European airports, while only 6 are located in the US. ${ }^{8}$ In the words of one commentator: "with the exception of the US, the system seems to be attractive to virtually all parts in the world."

The major stakeholders in slot allocation are: the airlines and other aircraft operators that want to use landing or takeoff slots of the airport, airport managing bodies responsible for the airport facilities, air traffic control authorities that provide the respective airport and airspace services, and, government authorities responsible for an airport. When an airport is classified as congested, the WSG requires the creation of a new role: a facilitator for Level 2 or a coordinator for Level 3 airports. These roles will be discussed in greater detail below.

\footnotetext{
${ }^{4}$ Katja Brecke, “Airport Slot Allocation: Quo Vadis, EU?,” Air \& Space Law, vol. 36(3) (2011): pp. $183-200$.

5 IATA, "Worldwide Slot Guidelines (WSG)," (August 2014, 6th Ed.), § 1.1, http://www.iata.org/policy/slots/Documents/wsg-6.pdf.

${ }^{6}$ Ibid., p. 1.

${ }^{7}$ IATA, WSG Annex, “Contact List for Level 2/3 Airports,” $§ 11.12$ (December 19, 2014), http://www.iata.org/policy/slots/Documents/wsg-annex-11.12.xlsx.

${ }^{8}$ Two Level 3 airports (JFK and EWR) and four Level 2 (ORD, LAX, MCO, and SFO).

${ }^{9}$ Claus Ulrich, "How the present (IATA) Slot Allocation Works" in Achim I. Czerny, Peter Forsyth, David Gillen, and Hans Martin Niemeier, eds., Airport Slots (Hampshire: Ashgate Publishing 2008), p. 19.
} 


\section{Governance Through the WSG}

As mentioned in the previous section, the WSG were developed by the IATA in 1976. Today, the IATA represents 240 airlines who carry $84 \%$ of total global air traffic, and who all adhere to the WSG. ${ }^{10}$

\section{A. Mission and Function}

The function of the WSG is to define the allocation and management of airport capacity in order to harmonize slot allocation standards. According to the Guidelines, "the WSG provides a consistent, transparent and fair method for the allocation and management of airport capacity. Air transport is global in nature and requires harmonized slot allocation standards at both the origin and destination airports of each route in order to maximize an airline's efficient use of resources."

Transparency is one of the key principles of the WSG. ${ }^{12}$ In particular, the created roles of coordinator and facilitator have an obligation of acting in a transparent way. ${ }^{13}$ Several transparency mechanisms are designated by prescribing in detail the information to be published (for example, regarding slot exchanges and slot transfers). Efficiency is another cornerstone of the WSG. It is mentioned several times in key provisions describing the purpose of the WSG: "The prime objective of airport coordination is to ensure the most efficient use of airport infrastructure in order to maximize benefits to the greatest number of airport users." 14 and "Air transport is global in nature and requires harmonized slot allocation standards at both the origin and destination airports of each route in order to maximize an airline's efficient use of resources." 15 The rules take into account efficiency both from airlines' and airports' points of view.

Accessibility to the WSG is an important element, mentioned in the first sentence of the document: "The WSG is organized and presented in a way to allow easy access to the policies, principles and processes that support the allocation and management of airport slots at congested airports worldwide." Specific accessibility rules are enshrined, (e.g., the access to databases on websites). ${ }^{16}$ The document does not mention legitimacy explicitly, although several rules seem to incorporate legitimacy indirectly. Understanding legitimacy as the appropriate and accepted use of power and authority for the common good, it seems that the WSG departs from the assumption that its actions are legitimate. Also participation is not referred to explicitly, but there are detailed rules to structure participation, which are explored below.

Other important principles are those of consistency, fairness, and non-discrimination, which are mentioned throughout the WSG document. The slot allocation itself is based on four principles: certainty of access, flexibility to mix and match slots to meet operational challenges and changing market needs, sustainability of costs, and transparency of allocation.

\footnotetext{
${ }^{10}$ IATA, “About Us,” http://www.iata.org/about/Pages/index.aspx.

${ }^{11}$ IATA, WSG $§ 2.1 .4$ (emphasis added).

12 "The WSG provides a consistent, transparent and fair method for the allocation and management of airport capacity." IATA, WSG $\S 2.1 .4$ (emphasis added).

${ }^{13}$ IATA, WSG $\S \S 4.2 .3$, and 5.2.3.

${ }^{14}$ Ibid., $\$ 1.2 .1$ (emphasis added).

${ }^{15}$ Ibid., § 2.1 .4 (emphasis added).

${ }^{16}$ Ibid., § 9.9.6 (emphasis added).
} 


\section{B. The Functional Solution to Overcome Infrastructure Capacity Overloads}

The WSG operates by designating particularly congested airports, creating the roles of airport coordinators and facilitators and laying down management principles for slot allocation at these airports. In order to identify critical spots in the network, airports are categorized as Level 1, 2, or 3 depending on capacity constraints:

- Level 1: airports where the capacity of the airport infrastructure is generally adequate to meet the demands of airport users at all times.

- Level 2: airports where there is potential for congestion during some periods of the day, week, or season which can be resolved by voluntary cooperation between airlines. A facilitator is appointed to facilitate the planned operations of airlines using or planning to use the airport.

- Level 3: airports where capacity providers have not developed sufficient infrastructure, or where governments have imposed conditions that make it impossible to meet demand. A coordinator is appointed to allocate slots to airlines and other aircraft operators using or planning to use the airport as a means of managing available capacity. ${ }^{17}$

"Participation" in the slotting process in the first instance depends on having been classified as a congested airport, a classification which is carried out according to the rules created by the WSG. If an airport is designated as Level 3, an airport coordinator is nominated, while for Level 2 airports a facilitator is appointed. The designation of a Level 3 airport is carried out in "full consultation with all stakeholders and interested parties." ${ }^{18}$ According to the WSG, stakeholders are:

- Airlines and other aircraft operators using or planning to use the airport.

- The airport managing body that administers and manages the airport facilities.

- The air traffic control authorities responsible for the airport and airspace.

- The coordinator or facilitator responsible for coordination at the airport.

- The government authorities responsible for the airport ${ }^{19}$

The airport managing bodies are responsible for fixing appropriate coordination parameters. This means fixing the hourly slots at an airport, in total movements and respective arrivals and departures. Capacity is one parameter, constrained by, for example, runway capacity, number of parking positions, waiting room capacities, and number of gates. ${ }^{20}$ However, the coordination parameters also reflect other constraints such as noise or environmental regulations. ${ }^{21}$

The WSG creates the role of airport coordinators and facilitators. A facilitator is defined as "the organization or individual responsible for collecting data on planned operations at a Level 2

${ }^{17}$ Ibid., $\S 1.4$.

${ }^{18}$ Ibid., $\$ 1.5$.

${ }^{19}$ Ibid., § 1.3 .

${ }^{20}$ Claus Ulrich, "How the present (IATA) Slot Allocation Works" in Achim I. Czerny, Peter Forsyth, David Gillen, and Hans Martin Niemeier, eds., Airport Slots (Hampshire: Ashgate Publishing 2008), p. 11.

${ }^{21}$ Ibid., pp. 11-12. 
airport, and recommending voluntary schedule adjustments as necessary." Coordinators are defined as: "The organization or individual responsible for slot allocation at a Level 3 airport."22

Coordinators must be functionally and financially independent of any single interested party and act in a neutral, transparent and non-discriminatory way. ${ }^{23}$ They are appointed in consultation with the airport managing body, the airlines using the airport and their representative organizations such as IATA. ${ }^{24}$ The role of the coordinator is described in detail in the WSG. ${ }^{25} \mathrm{~A}$ supporting document contains suggestions for the best practices for the functional and financial independence of coordinators: ${ }^{26}$

\subsubsection{The coordinator will:}

- Allocate slots to airlines and other aircraft operators in a neutral, transparent and non-discriminatory way, on the basis of the applicable coordination parameters, and in accordance with the priority criteria of the WSG and any local guidelines and regulations.

- Make available to interested parties details of the applicable coordination parameters, local guidelines and regulations, and any other criteria used in the allocation of slots, at least 7 days before the Initial Submission Deadline for each $\mathrm{SC}$, where possible.

- Make available to the airlines, ideally in SSIM Chapter 6 format and by online means, lists of slots allocated, remaining slots available and the reasons why slots were not allocated as requested.

- Attend and participate in all IATA SCs.

- Monitor cancellations made after the Historic Baseline Date and any nonutilization of slots for the purpose of applying the Use it or Lose it rule.

- Monitor planned and actual use of slots to identify any possible instances of intentional misuse of slots, and initiate a dialogue with the airline or aircraft operator concerned.

- Offer advice to airlines and the relevant authorities on all matters likely to improve airport capacity or slot allocation flexibility, and in particular on any area which will help the airport return to Level 2 or Level 1.

- Address problems arising from conflicting requirements in such a way as to avoid any need for external intervention. ${ }^{27}$

Coordinators have specific transparency obligations: "In the interests of fairness and transparency, coordinators must make available, upon request, the following information for review by all interested parties: (a) Historical slots by airline and chronologically for all airlines. (b) Requested slots (initial submissions) by airline and chronologically for all airlines. (c) All allocated slots, and outstanding slot requests, by airline and chronologically for all airlines. (d) Remaining slots available. (e) Comparisons between (a) and (c) above by time interval

${ }^{22}$ IATA, WSG, "Terms and Abbreviations," p. 53.

${ }^{23}$ Ibid., $\S 1.7 .1$.

${ }^{24} \mathrm{Ibid}$., $\S 5.2 .1$.

${ }^{25}$ Ibid., $\S 5.5$.

${ }^{26}$ IATA, WSG Annex, "Independence of Coordinator," § 11.14 (August 2014, 6th Ed.), https://www.iata.org/policy/slots/Documents/wsg-annex-11.14.pdf.

${ }^{27}$ IATA, WSG § 5.5.1. 
coordinated and by airline. (f) Full details of the constraints being used in coordination. (g) Full details of the criteria being used in the allocation of slots." 28

A Coordination Committee is established at Level 3 airports to advise the coordinator on matters relating to capacity, slot allocation and monitoring the use of slots at the airport. Membership of the Coordination Committee is open to:

- All airlines using the airport regularly and their representative organizations.

- The airport managing body.

- Air traffic control authorities.

- Representatives of general/business aviation (where relevant). ${ }^{29}$

These parties have relatively far-reaching consultation rights that are explicitly enshrined in the WSG. At the internal airport level, participation is contingent on a link to the airport. That link is defined as use for airlines or responsibility for air traffic control.

There are formal dispute resolution mechanisms, for example, in case of an airline's disagreement with a coordinator's determination of historical slots. In the very institutionalized slot negotiation process a deadline is set ("Agreed Historics Deadline") by which such a disagreement must be raised. Airlines can seek mediation by the Coordination Committee.

The WSG do not provide for public enforcement mechanisms. It is the national governments that set enforcement tasks. For example, in Germany violations of slot allocations are classified as "Ordnungswidrigkeiten" (a type of administrative offense) and subject to an administrative fine. ${ }^{30}$ However, enforcement remains a weak spot: "it has been pointed out that the existing slot allocation procedures lack the sanction mechanisms which could disincentivize some air operators to adopt practices that decrease the efficiency of the slot allocation process, such as overbidding of slots, late return of slots, and underutilization. These deficiencies need to be addressed."31

\section{The Allocation Process}

The process of allocating slots is highly formalized, the main event being the Slot Conference. In particular, the WSG lay down the operating procedures for the IATA Slot Conference. The conference is a physical meeting of the network participants, which takes place semi-annually (June and November) in different locations across the globe. The sole purpose of the Slot Conference is allocating and managing slots at Level 3 airports and discussing schedule adjustments at Level 2 airports. ${ }^{32}$ It is subject to strict timing through the Calendar of

\footnotetext{
${ }^{28}$ IATA, "Frequently Asked Questions: Slot Allocation Process-Is a Coordinator obliged to provide data from its database to any airline requresting such information?," http://www.iata.org/policy/slots/pages/faq.aspx.

${ }^{29}$ IATA, WSG $\S 5.6$.

${ }^{30}$ Bundesministerium der Justiz, "Verordnung über die Durchführung der Flughafenkoordinierung vom 13. Juni 1994 (BGB1. I S. 1262), die zuletzt durch Artikel 16 des Gesetzes vom 29. Juli 2005 (BGBl. I S. 2424) geändert worden ist," http://www.fhkd.org/images/pdf/FHKV.pdf.

${ }^{31}$ International Civil Aviation Organization (ICAO), "Worldwide Air Transport Conference, $6{ }^{\text {th }}$ Meeting: Slot Allocation,” Working Paper 11, ATConf/6-WP/11 (December 10, 2012), http:/www.icao.int/Meetings/atconf6/Documents/WorkingPapers/ATConf6-wp011_en.pdf.

${ }^{32}$ IATA, WSG $\S 2.2 .2$.
} 
Coordination. Two IATA Scheduling Conferences are held per year. As an example, the preparatory activities with correlated deadlines are given below:

- 15 September 2014 SHL Deadline

- 2 October 2014 Agreed Historics Deadline

- 09 October 2014 Initial Submission Deadline

- 28 October 2014 AppCal opened to Coordinators

- 30 October 2014 SAL Deadline

- 03 November 2014 AppCal opened to Airlines

- 11 November 2014 IATA Slot Conference

- 20 January 2015 Slot Return Deadline

- 31 January 2015 Historics Baseline Date

All airlines (IATA and non-IATA) with operating licenses can participate in the Slot Conference. Airline delegates must be fully authorized by the airline management to bind their company at the negotiations of the Slot Conference.

\section{Enablers}

The bi-annual Slot Conferences and their institutionalization through the surrounding schedule/process and digital communication tools are the main enablers in the domain. The Slot Conference is a forum in which the two main stakeholder groups meet (the airlines and the airport coordinators). It is embedded in tight schedules of post and ante event activities. Above all it results in a better distribution of information about demand and supply, and in some sense creates a slot market similar to a stock exchange.

There are several tools that enhance the physical conference. For example, there is a conference web tool called AppCal, a Standards Schedules Information Manual (SSIM), and an IATA online portal known as SlotLink. AppCal is the IATA's web-based appointments calendar tool. It facilitates the IATA Slot Conference appointment making process among participants and creates a meeting agenda prior to Conference commencement. The Standards Schedules Information Manual contains standards and procedures for schedules data exchanges. ${ }^{33}$ The IATA Schedules Information Standards Committee maintains the manual. IATA also maintains a workspace for airline scheduling activities using SlotLink. It serves as a support for the Slot Conference, as a communication forum for industry stakeholders, holds a library, and hosts the IATA workgroup dedicated to Slot Standards \& Policies. Additionally, the IATA provides introductory and advanced trainings to new attendees of the Slot Conference and participants in the airport coordination process.

The WSG are a standard. What makes the Worldwide Slot Guidelines particularly interesting is the way in which they operate. They are embedded in a tight network of institutional structures and formalized processes, which provide the real life traction of the mere document text itself. The role of the Slot conference must be highlighted in this respect. It is a "formalized" informal

\footnotetext{
33 "To facilitate the exchange of data, the IATA Member Airlines initiated the development of an official set of Recommended Practices to guide the industry along mutually compatible lines for schedule data handling procedures. These Recommended Practices and associated industry code sets are published in the Standard Schedules Information Manual (SSIM)." See IATA, "Standard Schedules Information Manual," Ref. No. 9179-21 (March 2011), http://www.cohor.org/wp-content/uploads/2013/04/SSIM-March-2011.pdf.
} 
contact platform. As commentator Claus Ulrich puts it: "experience has shown that even in the age of electronic communication, personal meetings are extremely useful for achieving solutions, and this forum is also being used for discussions among airlines themselves, eventually to exchange ('swap') slots, or for discussions among different airport coordinators in order to jointly find solutions for international flights." 34

\section{Governance of the WSG}

The WSG are overseen by the Joint Slot Advisory Group (JSAG), which is an IATA forum. The structure of the slotting governance network is concentrated in two global stakeholder institutions: (1) the airlines in IATA, in particular the Slot Policy Working Group, and (2) the airport coordinators in the World Wide Airport Coordinators Group (WWACG). These two global stakeholder institutions come together in the JSAG, with a delegation of seven airline members and seven airport coordinators.

\section{A. IATA/Slot Policy Working Group}

IATA membership is open to airlines operating scheduled and non-scheduled air services that hold an IATA Operational Safety Audit (IOSA) registration. IATA is now largely funded commercially through marketing its own products and services, but "remains devoted to its nonfor profit operations principles. ${ }^{35}$ It is incorporated in Canada. ${ }^{36}$

IATA has set up the Slot Policy Working Group (SPWG). The mandates are laid down in the Working Group's Term of References:

- Development of amendments to the World Wide Slot Guidelines.

- Represent IATA Members at the Joint Slot Advisory Group (JSAG) that meets regularly with airport coordinators to discuss matters of mutual interest concerning the Slot Conference and slot management matters.

- Provide technical guidance to IATA dialogue with regulatory and other interested parties on schedules and slot matters.

- Provide guidance to IATA in the restructuring of the Slot Conference into a cost effective and efficient process that delivers timely and valued service to IATA Members and the industry as a whole. ${ }^{37}$

\section{B. World Wide Airport Coordinators Group}

The WWACG membership is open to all coordinators (natural or legal persons) of Level 3 designated airports and to schedules facilitators of Level 2 airports under the WSG. The WWACG is funded by its members. The members are national coordination or schedules facilitation organizations. These organizations' member structures and national airline and airport participation are extremely diverse and subject to differing funding arrangements. For

\footnotetext{
${ }^{34}$ Claus Ulrich, "How the present (IATA) Slot Allocation Works" in Achim I. Czerny, Peter Forsyth, David Gillen, and Hans Martin Niemeier, eds., Airport Slots (Hampshire: Ashgate Publishing 2008), p. 16.

${ }^{35}$ IATA, "About Us_-From a New Trade Association to a New Strategic Trust," http://www.iata.org/about/Pages/history_5.aspx.

${ }^{36}$ For the Act of Incorporation, Articles of Association, Rules, Boards and Composition see IATA, "IATA's Corporate Governance Structure," http://www.iata.org/about/pages/corporate-structure.aspx.

${ }^{37}$ IATA, "Medical Advisory Group_Terms of Reference" (October 2013), http:/www.iata.org/about/Documents/working-groups-terms-of-reference-membership.pdf.
} 
example COHOR: Airport Coordination, France has a budget of $€ 1.44$ million for one fiscal year, and is funded partially by airport management bodies and airlines. Other entities are also partially state funded. The organization comprises of 12 air carriers and 3 airport managing bodies, including Aéroports de Paris, Aéroport de Lyon, and Aéroport de Nice. ${ }^{38}$

\section{The Joint Slot Advisory Group}

The JSAG brings together these two global stakeholder institutions, SPWG and WWACG. It is an IATA forum for member airlines and airport coordinators to discuss issues of common concern and oversee the WSG. The JSAG also advises on the IATA Slot Conference organization. Recommendations of JSAG to IATA are "based upon consensus between JSAG members and input from the IATA Slot Policy Working Group (SPWG) and the Worldwide Airport Coordinators Group (WWACG)." ${ }^{39}$ Changes agreed to by the JSAG are presented to the Heads of Delegation of the Slot Conference for majority endorsement.

Within the JSAG, the airlines' representatives are a subgroup of the SPWG, appointed by the IATA Director General, from a list of member employees having expressed an interest and demonstrated skill and experience. ${ }^{40}$ Looking at the composition of airline members, large and prestigious airline companies have been chosen. For example, members include Air France, All Nippon, American Airlines, British Airways, Deutsche Lufthansa AG, KLM, United Air Lines.

The representative of the airport coordinators comprise the WWACG Core Group, which consists of seven members - elected for two-year terms (renewable) - from within the WWACG that represents the Airport Coordinator Community. The JSAG meets as necessary, but one meeting each year is to be held at the Geneva or Montreal offices of the IATA.

According to the JSAG terms of reference "every effort will be made to achieve a broad geographical representation of airlines and coordinators." 41 The geographical spread of members has roughly one representative per continent, with the exception of Europeans, who are overrepresented.

From a governance point of view it is interesting to note that the WSG created the roles of coordinators and facilitators, which are now a significant stakeholder institutions governing the WSG standardization process.

\section{Issue Identification and Change}

The WSG are understood as a living document. This means that they change the rules of slot allocation to address changing needs and new practices of the community. Changes can be suggested by filling in a simple document that is available on the Internet, indicating the summary of the proposed change, reasons for the change, proposed amendment to the text, and implications or impact. ${ }^{42}$ The proposal is then discussed within the JSAG meetings. Initial issue

\footnotetext{
${ }^{38}$ See World Wide Airport Coordinators Group (WWACG), “Organizing \& Funding Arrangements of WWACG Members," http://www.wwacg.org/FTableList.aspx?list=11.

${ }^{39}$ IATA, WSG Annex, "Joint Slot Advisory Group-Terms of Reference," § 11.6, http://www.iata.org/policy/slots/Documents/wsg-annex-11.6.pdf.

${ }^{40}$ See IATA, WSG Annex, "Slot Policy Working Group (SPWG) Terms of Reference," $§ 11.5$, http://www.iata.org/policy/slots/Documents/wsg-annex-11.5.pdf.

${ }^{41}$ IATA, WSG Annex, $\S 11.6$.

${ }^{42}$ See IATA, "Proposal for Revision of the WSG," http://www.iata.org/policy/slots/Documents/pro-rev-cur-
} 
identification is undertaken mainly through the slot aviation community, for example, by providing easy access for change proposals.

Functionally, the issues identified related to slot allocation are dealt with in an exclusively technical fashion. Perhaps one could advance the claim that topics are artificially made more technical in order to circumvent their political nature. Certainly slot allocation as an issue has been isolated from other aviation governance concerns and been given an autonomous governance network.

Two governance characteristics can be pointed out as particularly relevant: (1) creating a flexible system of rules that is organically mapped on existing processes and (2) the isolation of relevant technical aspects within an autonomous governance sub-system.

\section{Outcomes}

\section{A. Evaluation of the WSG Operation}

The outcomes of the WSG can be evaluated on two aspects. The first is functional, namely whether slot allocation alleviated airport bottlenecks, and whether it is indeed the optimal mechanism. The second is an evaluation of the governance processes, and whether the WSG has created a viable process for different stakeholders to participate in decision-making.

On a functional level, only congested airports are provided with a specific slot regulation. The solution to congestion issues is thus based on two phases: (1) the identification and classification of overloaded spots in the network (i.e., congested airports) and (2) the process for slot allocation, which is currently a negotiated quota system with the potential for a secondary trading market. While the system has provided a workable solution for the aviation domain, it has been subject to criticism, notably for its anti-competitive effects and rigidity, resulting in sub-optimal slot allocation. For example, it is argued that "grandfathering rights" ${ }^{43}$ result in a substantial burden on new entrants and prevent effective competition.

Several alternatives in this respect are discussed, namely congestion pricing, ${ }^{44}$ auctioning, ${ }^{45}$ and systems of secondary trading. It is questioned who actually owns slots-" "the Member State, the airport or the air carriers themselves, ${ }^{, 46}$ and whether the allocated slots are a transferrable property right. The problem of allocating slots for free is that, currently, airlines have no incentives to price slots, and acquisition of slots is done independently of slot valuation. In theory, as long as the "ownership" of slots is assigned, secondary trading would also lead to

wsg.doc.

${ }^{43}$ Grandfathering rights refer to the fact that new slot allocation is based on slot use historics for airlines.

${ }^{44}$ The congestion pricing model would reflect an increase in price with the level of congestion faced by an airport. However, information about the value of slots for airlines is not known, therefore the pricing would be difficult to undertake and likely lead to inefficiencies. See Jakub Kociubiński, "Regulatory Challenges of Airport Slot Allocation in the European Union," Wroclaw Review of Law, Administration \& Economics, vol. 3(1) (June 2013): pp. 28-46.

${ }^{45}$ Alternatives proposed are auctions, in which slots are sold at the best price. However, a simple method of auctioning likely inhibits important synergies in slot aggregation, although alternative forms of auctioning can address these issues effectively. Jakub Kociubiński discusses for example the Vickrey-Clarke-Groves system and Cryptographic Combinatorial Clock-Proxy. See Kociubiński, "Regulatory Challenges," (June 2013).

${ }^{46}$ Kociubiński, "Regulatory Challenges," (June 2013), p. 32. 
efficient market outcomes. Although a slot market may suffer certain imperfections, notably information asymmetries, it is generally judged to be capable of fostering competition and hence a more efficient allocation. ${ }^{47}$ Thus, it is argued that a system of secondary trading could complement the official WSG slot allocation system.

A second problem that surfaces with respect to secondary trading is the interactions between different governance spheres, as it is geographically regulated and practiced in different ways. Secondary trading under the EU rules is currently an area of legal uncertainty, but does occur in the UK ${ }^{48}$ Under EU law, secondary trading was subject to some legal challenges, but ultimately is tolerated. Additionally, the EU Commission has proposed a regulation that would formalize the secondary trading option.

\section{B. Stakeholder Views}

In the European context, the EU has conducted a wide stakeholder consultation. It needs to be understood as having several limitations: it is a geographically confined overview, although several non-European parties made submissions and - secondly-concerns the EU regulation on slotting. Although the rules correspond to a very high degree with those of the WSG on which they are based, the results reflect the industry positions on the slotting system. From this very wide stakeholder consultation, the following industry positions appear:

- Airline and airline associations overall seem satisfied with the functioning of the system.

- Concerns have been expressed that slots needed capacity expansion rather than management.

- Airlines can be split up into different types of operators, for example, scheduled, charter and low cost operators, business aviation, and their associations. These do not always have homogenous interests: business aviation operators, for example, argued that business aviation is unfairly disadvantaged, as at most EU airports it is not possible to gain historic rights to slots on the basis of total operations. ${ }^{49}$

Airports and airport associations seemed to be adopting a more operational take on the evaluation of the slotting mechanism, most often indicating specific aspects to be improved. These consisted notably of the need to move from an administrative model towards an efficiency model. An example cited was "by encouraging larger aircraft or by enhancing incentives and sanctions to increase the actual usage of allocated capacity." 50 The main problematic points identified were the ownership of slots, the improvement of the transparency of slot allocation, and the question of secondary trading.

\footnotetext{
${ }^{47}$ Ibid., pp. 42-43.

48 "Such a market in airport slots (in the form of secondary trading) has been in operation at UK airports for some time, as the Commission recognised in a 2008 Communication. Indeed, slots at London Heathrow have changed hands for high prices: in March 2008 it was widely reported that Continental Airlines had paid \$ 209 million (or $€$ 143 million at the then exchange rate) for four pairs of slots at Heathrow." European Commission, "Proposal for a Regulation of the European Parliament and of the Council on common rules for the allocation of slots at European airports," COM(2011) 827 final (Brussels, 1.12.2011), http://eur-lex.europa.eu/legalcontent/EN/TXT/?uri=CELEX:52011PC0827.

${ }^{49}$ Steer Davies Gleave (European Commission Study), "Impact Assessment of Revisions to Regulation 95/93Results of Stakeholder Consultation," Prepared for European Commission DG MOVE DM24 Brussels B-1049 (March 2011).

${ }^{50}$ Ibid.
} 
Transparency and the independence of coordinators were issues that were repeatedly referred to in an EU consultation. Notably, the independence of coordinators and mechanisms in place to ensure this independence were questioned by airline and airline associations: "Many carriers highlighted the differences in the level and standard of information provided in different States, although most added that this information was still sufficient to plan their schedules.",51

In terms of governance, however, the system has gradually institutionalized a negotiation process that grew out of the need for slot allocation. It is a very mature constellation of stakeholders, which has grown organically according to the needs identified since 1976. By creating the roles of coordinators and facilitators, the WSG gave a voice to airports that is functionally limited to the specific issue of slot allocation. Effectively, the airline industry created its own interlocutor at airports. Now, aviation slot governance is characterized by an intense concentration of two main stakeholder camps, namely airports on the one hand and airlines on the other. This institutional concentration of main stakeholders is the pillar upon which aviation slot governance rests.

\section{Cross-Sphere Synchronization}

The number of capacity constrained Level 3 airports has risen constantly, from 136 in 2000, to 155 in 2010, and to 167 in $2014 .{ }^{52}$ Of these, 104 airports are situated in Europe, of which 92 are in the $27 \mathrm{EU}$ Member States. 43 are in Asia Pacific, and only 11 are scattered between the Middle East, North America, and South Africa. ${ }^{53}$ The geographical distribution indicates that the process of slot allocation is much more important for aviation in Europe than it is in other areas, such as the United States.

The geographical spread is reflected in the personnel at all levels of the global slot governance network. The organization of the network is multilayered, and made up of regional and national umbrella organisations.

Taking the example of the EU, the European Airport Coordinators Association (EUACA) is the European level counterpart of the worldwide airport coordinators group. Membership of the EUACA comprises coordinators and schedules facilitators within the EU and the European Economic Area (EEA) or otherwise legally bound by the European Economic Community (EEC) Regulation 95/93 either as individuals or as representatives of their organisations. At the national level, the umbrella structures are very diverse in terms of membership. In particular, the degree to which a national level coordinator cooperative is made up of different airports or simply the most important one is dependent on how many Level 3 airports exist in a country.

Importantly, as the WSG recognizes, states or regions may have deviating regulations, which take precedence over the industry standard. There is always the risk that legislation trumps the WSG.

A current issue is, for example, the proposal to revise the slots EEC Regulation No 95/93 on the allocation of slots at community airports, which has caused a discrepancy between the IATA position and the Commission proposal. The IATA's main European Regional Office and the

\footnotetext{
${ }^{51}$ Ibid., at 1.49

${ }^{52}$ IATA, WSG Annex, "Contact List for Level 2/3 Airports," § 11.12 (December 19, 2014); ICAO, "Worldwide Air Transport Conference, $6^{\text {th }}$ Meeting: Slot Allocation," Working Paper 11, ATConf/6-WP/11 (December 10, 2012).

${ }^{53}$ Ibid.
} 
Operations and Service Center are located in Madrid. The IATA maintains relations with governments, and intensively monitors EU developments. The IATA prepared a frank position paper, in which it stated its disagreement with several of the proposals made by the European Commission. The European Parliament has backed the IATA position in several respects, so that the European Commission's proposal will likely be amended to be more in line with the IATA position.

While local regulations trump the non-binding WSG standards, they can also reinforce the standard. This is the case, for example, in the EU, which makes most elements of the WSG binding through legislation. At the same time, this national or regional legislative interface also acts as a check-and-balance, as the legislative process results in a critical examination of the private rules.

\section{Conclusions}

Structurally, the aviation domain is made up of the following actors: states, designated air navigation service providers, ancillary service providers, airport operators, airlines, the manufacturing industry, and passengers. Slot allocation is a "private" island in a largely public aviation domain as it brings together the main private stakeholders, namely airlines and airports.

In terms of governance, what kind of solutions does the WSG present? (1) The identification of bottlenecks, and devising a commonly accepted methodology for doing so. The classification of critical allocation spots in the network became institutionalized. An airport is classified as Level 3 following a demand and capacity analysis, carried out according to the rules laid down in the WSG. The slot allocation process is a tiered process in that airports are classified in one of three categories of capacity constraints. In this sense, the greater the problem, the greater participation in the solution process. After the identification of critical spots, the solution presented by the WSG (2) is the creation of new management roles within the system, and a concentration of two main groups of stakeholders. The creation of new roles (airport coordinators and airport facilitators) leads to formalized representation of airports. The roles are (3) integrated in $a$ formalized negotiation and solution finding process. The WSG streamlined and formalized processes which had informally developed and created a successful forum, namely the IATA Slot Conference.

The main criticism targeted towards the WSG-system is that it commits to an administrative mechanism, which - while providing a stable solution to the issue of the allocation of slots - may provide sub-optimal allocation with regard to efficiency. The WSG does not assign the slots to those parties that would value them most. It is possible that an increase in efficiency could be obtained if the slots were able to be traded, and therefore a valuable object of property rights. 


\section{Appendix B:}

The Evolution of Governance Structure in Cryptocurrencies and the Emergence of Code-Based Arbitration in Bitcoin 


\title{
NoC Internet Governance Case Studies Series: The Evolution of Governance Structure in Cryptocurrencies and the Emergence of Code-Based Arbitration in Bitcoin
}

\author{
Markus Oermann and Nils Töllner \\ Hans-Bredow Institute for Media Research
}

Editorial Note: Context, Character, and Purpose of the Case Study

This case study is part of a globally coordinated, independent academic research pilot project by the Global Network of Interdisciplinary Internet \& Society Research Centers (NoC). Facilitated by the Berkman Center for Internet \& Society at Harvard University, this study examines existing multistakeholder governance groups with the goal of informing the future evolution of the Internet governance ecosystem. Building upon the NETmundial Principles and Roadmap, it contributes to current policy debates at the international level, including the Internet Governance Forum, the NETmundial Initiative, and other organizations and efforts.

Internet governance is an increasingly complex concept that operates at multiple levels and in different dimensions, making it necessary to have a better understanding of both how multistakeholder governance groups operate and how they best achieve their goals. With this need in mind, at a point where the future of Internet governance is being re-envisioned, colleagues from several NoC institutions around the world have written twelve case studies examining a geographically and topically diverse set of local, national, and international governance models, components, and mechanisms from within and outside of the sphere of Internet governance. Key findings from these cases are summarized in a synthesis paper, which aims to deepen our understanding of the formation, operation, and critical success factors of governance groups and even challenge conventional thinking.

The research, based on twelve case studies, suggests that there is no single best-fit model for multistakeholder governance groups that can be applied in all situations. Rather, it reveals a range of approaches, mechanisms, and tools available for both the formation and operation of such groups. The analysis demonstrates that the success of governance groups depends to a large degree on the careful selection, deployment, and management of suitable instruments from this "toolbox." As governance groups pass through different phases of operation, conveners and facilitators must remain alert to changes in circumstances that necessitate adjustments to the approaches, mechanisms, and tools that they deploy in order to address evolving challenges from inside and outside. This case study provides insights into how those instruments can be deployed and adjusted over time within such groups, and highlights how their interactions with important contextual factors may be successfully managed within given resource restraints.

The research effort is grounded in a diversity of global perspectives and collaborative research techniques. Adhering to objective and independent academic standards, it aspires to be useful, actionable, and timely for policymakers and stakeholders. More broadly, the Network of Centers seeks to contribute to a more generalized vision and longer-term strategy for academia regarding its roles in research, facilitation and convening, and education in and communication about the Internet age.

For additional information on the initiative, please contact Urs Gasser, Berkman Center for Internet \& Society, at ugasser@cyber.law.harvard.edu. 


\begin{abstract}
This case study describes the process by which Bitcoin revised its core code to accommodate a new feature called "multi-signature transactions." Bitcoin is a cryptocurrency, which was introduced in 2009 and has spread rapidly since then. It is based on open-source peer-to-peer software that establishes a network of user accounts (wallets) in which the units of account (bitcoins) are produced and transmitted. It is a goal of cryptocurrencies in general to operate without a central agent, which makes it complicated to resolve disputes. To address this, Bitcoin added multi-signature transactions. This case study examines how that change was made. In particular the case study explores how an open source community is able to maintain a stable codebase that can serve as a basis for an entire form of currency, while still making necessary changes. The case shows that the decision-making processes regarding transformations of Bitcoin's governance structure are not as transparent as one might expect given the cryptocurrency's commitment to open source. However, major changes to the code are publicly discussed in the Bitcoin developers' community and the authors could not identify a single case of a decision in which the core development team deviated from the consensus of the community.
\end{abstract}




\section{Table of Contents}

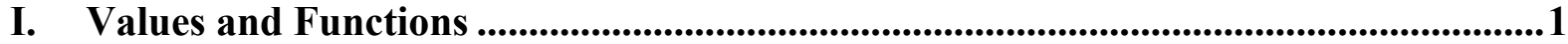

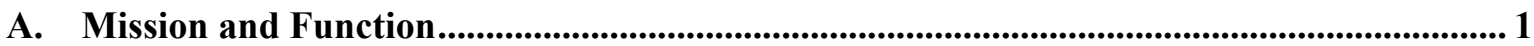

1. The Case: Emergence of Code-Based Arbitration in Bitcoin............................................... 1

2. A Heuristic Model of Governance Factors in Online Services .............................................. 4

II. Organizational Model and Structure ...................................................................6

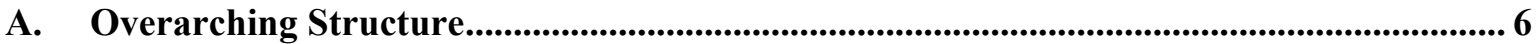

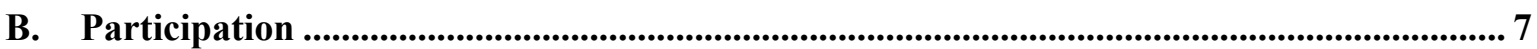

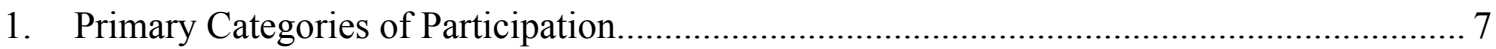

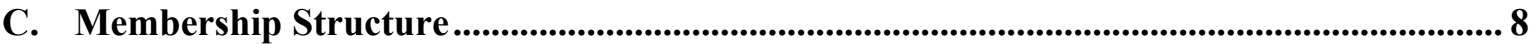

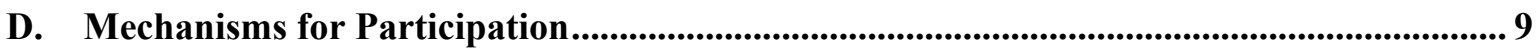

E. Decision-Making Structures ................................................................................................................ 10

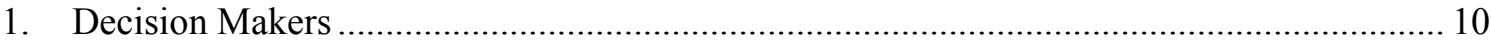

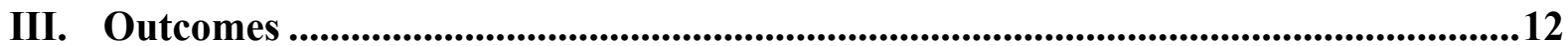

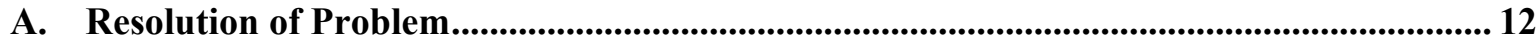

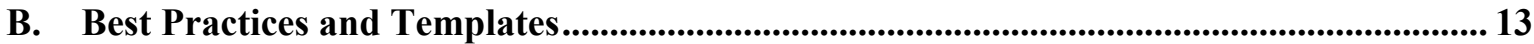




\section{Values and Functions}

\section{A. Mission and Function}

\section{The Case: Emergence of Code-Based Arbitration in Bitcoin}

When talking about the future of e-business, discussions often touch on the rapid spread of ecurrencies or cryptocurrencies over the last five years. In that short period of time, Bitcoin quickly gained prominence as the most well known example of this new means of payment. No longer just a niche technical proof-of-concept, these new payment systems can be used to buy a Dell computer or donate to the Wikimedia Foundation, among other things. ${ }^{1}$ For that reason, it is not unreasonable to say that they have become a real alternative to traditional "offline" currencies like the U.S. dollar, yen, or euro.

The rise of these cryptocurrencies has lent new urgency to the classic question of how to handle business conflicts. Due to some of the special conceptual and technological features that set them apart from traditional currencies, managing conflict can be a tricky matter when deals utilize cryptocurrencies instead of traditional currencies.

Bitcoin, which was introduced in 2009 and has spread rapidly since then, is based on opensource peer-to-peer software that leverages a network of user accounts (wallets) set up on peripheral sites in which the units of account (Bitcoins) may be stored after they are produced and transmitted. A public ledger (the blockchain) plays a central role in this system, tracking every transaction in which Bitcoins are exchanged while maintaining the anonymity of the users behind the exchange through public key cryptography. Because it is very hard to connect the public keys used to announce exchanges to the associated private keys used to verify them, the parties of a transaction are essentially anonymous. ${ }^{2}$ As the ledger is distributed among all the users in the network, ${ }^{3}$ no central administrator or repository is needed to run the system.

There are two ways to obtain Bitcoins. First, users can gain Bitcoins through "mining." Due to the cryptographic nature of the system and the complex algorithms underlying it, verifying a Bitcoin payment and recording it in the ledger requires massive amounts of processing power. Users can provide computing power to the network to verify payment transactions, and in exchange they awarded a proportionate amount of new Bitcoins, a process called mining. In other words, new Bitcoins are produced as a by-product of payment processing and given to the users who helped process that transaction. Given that the number of possible Bitcoins has been limited to 21 million by the software protocol, the stock of potential new Bitcoins gets smaller with every Bitcoin created and released into the system. The algorithm is designed so that the processing power required to mine a new Bitcoin increases with every new user entering the network, making new Bitcoins harder to create. Accordingly, the second way to get Bitcoins is

\footnotetext{
${ }^{1} C f$. http://en.community.dell.com/dell-blogs/direct2dell/b/direct2dell/archive/2014/07/18/we-re-now-acceptingbitcoin-on-dell-com.aspx (retrieved from September 10, 2014); http://blog.wikimedia.org/2014/07/30/wikimediafoundation-now-accepts-bitcoin/ (retrieved September 10, 2014).

${ }^{2}$ Problems of this privacy concept by pseudonymity are addressed by actual research, cf. e.g. Biryukov/Khovratovich/Pustogarov (2014): Deanonymisation of clients in Bitcoin P2P network, http://arxiv.org/pdf/1405.7418.pdf.

${ }^{3}$ As long as using the original client, every user stores a copy of it on her device, within her wallet.
} 
through trade. The typical way a new user obtains her first Bitcoins is by changing an offline currency into Bitcoins via a dedicated trading platform. ${ }^{4}$

Bitcoin has been widely criticized for its technological implementation, its potential for abuse in illegal online platforms like the anonymous black market site the Silk Road, and for the volatility of the Bitcoin market. ${ }^{5}$ This paper will not further discuss these criticisms nor develop its own line of reasoning regarding those arguments. This paper instead aims to examine the governance structures supporting the technical development of Bitcoin.

Before considering Bitcoin's governance structure, it is important to understand a goal central to cryptocurrencies in general, and Bitcoin in particular: these systems should work without a central agent in charge of enabling the payments and upon whose integrity and responsibility the system relies. Or, as Satoshi Nakamoto, the person who first proposed the concept of Bitcoin in a whitepaper in 2008, so aptly put it:

"The root problem with conventional currency is all the trust that's required to make it work. The central bank must be trusted not to debase the currency, but the history of fiat currencies is full of breaches of that trust. Banks must be trusted to hold our money and transfer it electronically, but they lend it out in waves of credit bubbles with barely a fraction in reserve. We have to trust them with our privacy, trust them not to let identity thieves drain our accounts. Their massive overhead costs make micropayments impossible. (...) With e-currency based on cryptographic proof, without the need to trust a third party middleman, money can be secure and transactions effortless."6

Eliminating the middleman, however, creates some challenges for Bitcoin and other cryptocurrencies. Without trusting a middleman, traditional avenues for business dispute resolution no longer work because the common enforcement "infrastructures" (e.g., state courts or private courts of arbitration) cannot direct an intermediary, like banks or credit card companies, to reverse the payment. Instead of relying on intermediaries, Bitcoin's design transfers all the control over a transaction to the network itself. When a user wants to make a payment, she uses the recipient's public key as a substitute for a bank account number and then initiates the transaction by signing off on it with her own private key. The recipient then accepts the transaction by signing using his private key. ${ }^{7}$ Undoing a transaction works similarly, making it impossible for an institution outside the system to revert a payment without the participation of the parties to the transaction.

Business transactions based on cryptocurrencies can result in conflict, just like business deals using any other currency. Imagine the purchase of a certain good, where the seller and buyer

\footnotetext{
${ }^{4}$ Cf. https://en.wikipedia.org/wiki/Bitcoin (retrieved September 10, 2014).

${ }^{5}$ Cf. Simonite, Tom (2013): Silk Road Bust Could Slow Bitcoin Economy, http:/www.technologyreview.com/view/519846/silk-road-bust-could-slow-bitcoin-economy/ (retrieved from September 10, 2014); Quiggin, John (2013): The Bitcoin Bubble and a Bad Hypothesis, $\mathrm{http}: / /$ nationalinterest.org/commentary/the-bitcoin-bubble-bad-hypothesis-8353 (retrieved from September 10, 2014); Matthew Sparkes, "Software activist calls for 'truly anonymous' Bitcoins to 'protect democracy'," The Telegraph (UK), December 2, 2013, http://www.telegraph.co.uk/technology/news/10488201/Software-activist-callsfor-truly-anonymous-Bitcoins-to-protect-democracy.html.

${ }^{6}$ Satoshi Nakamoto: Bitcoin open source implementation of P2P currency. 02-11-2009.

http://p2pfoundation.ning.com/forum/topics/bitcoin-open-source (retrieved September 10, 2014).

${ }^{7}$ Cf. https://bitcoin.org/en/how-it-works (retrieved September 10, 2014).
} 
disagree on its quality. The buyer is claiming deficiency, and the two sides seek to resolve the dispute, but neither party may want to involve official public institutions. ${ }^{8}$ How could this conflict be dealt with and resolved if the parties are unable to find a solution through negotiation alone?

Beginning in late 2013, a feature of the Bitcoin protocol offered a solution to this challenge: "multi-signature" transactions. Every Bitcoin transaction is defined in a script, which sets conditions on how a subsequent user can access the coins. Because these rules are defined in code, they can be adjusted. One adjustment is setting a minimum number of parties required to sign off on any given transaction. In a standard two-party deal, for instance, the script can define that the signatures of two out of three users are needed to complete the transaction. This enables the two primary parties to a deal (a buyer and seller) to name a third user as an arbitrator in case of conflict. Where there is no disagreement, the parties can process the payment on their own, and the arbitrator cannot hinder it. But in case of a conflict, one side can refuse to sign the payment and invoke the arbitrator. The arbitrator can resolve the conflict and enforce her ruling by signing off of the transaction or not. ${ }^{9}$ The feature of multi-signature transactions was actually included in the software protocol as a standard option in 2011-2012, but users largely ignored it because there was no representation in the graphical user interface until late $2013 .{ }^{10}$ Once the feature gained prominence, sites such as https:/www.bitrated.com emerged as platforms for parties to find independent arbitrators. A wide variety of people offer themselves on these platforms to be commissioned to act as arbitrators. ${ }^{11}$

This paper takes a deeper look at this change in Bitcoin's architecture. In our view, this is an interesting example of a structural evolution of a cryptocurrency system that we will analyze from a governance perspective.

When thinking about governance, we can examine phenomena of emergence, application and effects of collective norms and rules from at least three different angles. Which individual and collective actors form the governance group ${ }^{12}$ Based on the answer to this question we can take a look at how the power of influencing the normative content of the rules and their social realization is distributed among these actors. With an emphasis on processes, we can analyze if and how the production and stabilization of certain rule sets form new institutions and how these rule sets influence social reality by coordinating behavior. And third, we can focus on the norms and rule sets and analyze, across the factors of governance, which normative meanings they contain, and how the governance structure formed by them is configured.

\footnotetext{
${ }^{8}$ In fact, the Bitcoin Foundation recently published a primer for Bitcoin users on the basic legal requirements that cause a Bitcoin deal to activate a certain jurisdiction - and how to avoid this, $c f$. McFarlan, Robert A. (2014): $A$ Bitcoin Primer on Jurisdiction. https://bitcoinfoundation.org/wp-content/uploads/2014/08/Bitcoin-JurisdictionPrimer.pdf ( retrieved from September 10, 2014).

${ }^{9}$ Dourado, Eli (2014): Stop Saying Bitcoin Transactions Aren’t Reversible. http://elidourado.com/blog/bitcoinarbitration/ (retrieved from September 10, 2014).

${ }^{10}$ Cf. https://github.com/bitcoin/bips/blob/master/bip-0011.mediawiki (retrieved September 10, 2014); https:/github.com/bitcoin/bips/blob/master/bip-0019.mediawiki (retrieved September 10, 2014).

${ }^{11}$ More than 240 organizations or private persons offered to serve as arbitrators on bitrated.com in late September 2014.

12 "Governance group" refers to the social formation that produces, establishes, and applies the norms and rules in this context.
} 
This structural perspective supplies us with basic insights that lay the groundwork for the analysis from the other perspectives. We will start by clarifying the values and functions related to the change in Bitcoin's governance structure (A.1.2). Based on these reflections, we will shift our analytical focus to the governance group itself: what is its organizational model and structure? (B.). Then we will discuss from a procedural angle how actors could participate as members of this group (C.) and consider how decisions of and in the group are made (D.). In section $\mathbf{E}$. we sum up the outcomes of our analysis and present the key lessons and takeaways from this case study.

\section{2. $\quad$ A Heuristic Model of Governance Factors in Online Services}

It is useful to consider the governance of Bitcoin using a heuristic model of governance factors impacting user behavior in online services in general. This model differentiates between the components of code, state law, contracts and social norms. ${ }^{13}$

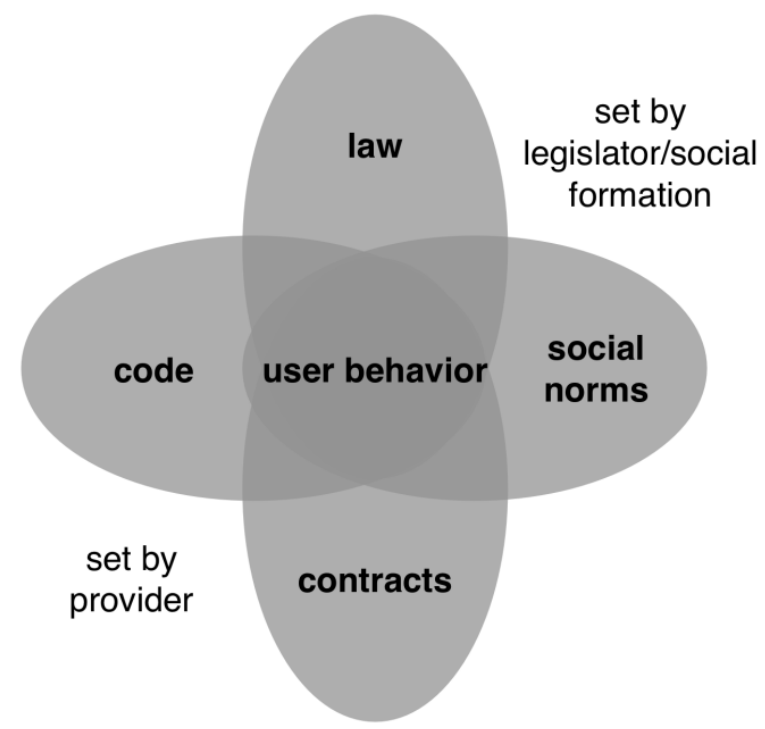

Figure 1. Oermann/Lose/Schmidt/Johnsen (2014), note 12: fig. 1, p. 18.

"Social norms" in this heuristic refer to all of those rules emerging from general tacit conventions in a society or norm sets developed by and applied in a specific social formation, such as the community of users of a certain online service. We sometimes find these norms codified as codes of conduct or (n)etiquette. These social norms are both established and executed by the social formation, which reacts to violations with social sanctions. In contrast, "state law" contains provisions for user behavior in online services stipulated by the state in

\footnotetext{
${ }^{13}$ Deviating from Lessig's model we do take the "market" into account as a factor of governance because a "market" lacks in itself a normative dimension from our point of view. Saying that "markets" are comparable to law and code analytically could lead to misunderstandings because it could invite comparisons between the "natural laws" of markets of macroeconomic theory and the "designed" norms of law and code. We think that it is more fruitful, therefore, to look at social norms, which function as a transmitter of normative predictions which have been associated with "markets" when approaching governance in online services on a structural level. For a more detailed explanation of this argument, $c f$. Oermann, Markus/Lose, Martin/Schmidt, Jan/Johnsen, Katharina (2014):

Approaching Social Media Governance. Berlin, HIIG Discussion Paper Series, (May 2014), http://papers.ssrn.com/sol3/papers.cfm?abstract_id=2498552 (retrieved September 10, 2014).
} 
codified legal norms, which are enforced by state institutions like courts and public authorities. "Contracts" in the case of online services are found mostly in the form of Terms of Service (TOS) and are for the most part laid down unilaterally by the service providers and executed through mechanisms like content removal.

While the above three forces on user behavior are fairly self-explanatory, the concept of "code" in this model is less clear. Harvard Professor Lawrence Lessig applied this concept to online user behavior in 1999 with his famous analogy "code is law."14 However, there still is no widely shared understanding of what is meant when we talk about "code" in the normative context of governance. Surely it is not just software source code, because software requires hardware in order to shape user experience. So we have to take the aspect of hardware into account, too. Furthermore, the source code alone does not help us understand how certain technology is used, nor does it explain the effect that technology will have on user behavior on a structural level. But we can look at the interfaces of technology and human behavior in order to analyze the affordances that the code (hardware and software) offers the user and the constraints it sets to them. ${ }^{15}$ For our purposes, therefore, we refer to code as all the normative aspects of a certain technology, which encompasses more than simply source code.

So far we have established that all four concepts introduced above share a common quality: they constrain and shape user behavior. These predictions differ in their normative strength. If social norms, law, contracts or code provide the addressee with several possibilities for action ("If $\mathrm{x}$ is the case, you can do a, b, or c..."), they can be said to have minor normative strength. On the other hand, if they prescribe just a single course of action ("If $\mathrm{x}$ is the case, you must do y."), they have maximum normative strength. Of course, all gradations of normative strength are also possible for each of the four factors. The four concepts also differ concerning the consequences in case of deviating behavior. If we look at phenomena of human interaction in online services, we will always find a complex structured normative background formed by these factors, on which the application potential of the services are realized by the users and on which their behavior is coordinated. This is what we call the governance structure. ${ }^{16}$

If we now consider Bitcoin in light of this model, we see that this cryptocurrency is conceptualized in a way that three of the above factors should and actually do just play a minor role in its governance structure - at least as far as this is possible without endangering the functionality of Bitcoin as a means of payment:

- There should be no influence of law: Control by the state on payments, transactions and the underlying business deals should be prevented;

- Social norms should be also prevented from having an influence on the behavior of Bitcoin users, at least as far as they concern trust in the institutions that act as the

\footnotetext{
${ }^{14}$ Lessig, L. (1999): Code and Other Laws of Cyberspace. New York, U.S; Lessig, L. (2006): Code: And Other Laws of Cyberspace, Version 2.0. New York, U.S.

${ }^{15}$ Oermann, Markus/Ziebarth, Lennart (2015): Use of Cultural Artifacts by Way of Interpretation and Applicationor: Adapting the Methodology to Analyze the Normative Contents of Law for the Analysis of Technology.

Computer Law and Security Review, vol. 32(1) (forthcoming).

${ }^{16} \mathrm{Cf}$. Oermann, Lose, Schmidt, and Johnsen (2014), supra note 12, at 8-17.
} 
conduits for offline currencies. Bitcoin is designed to prevent any connection to the traditional, regulated financial system; ${ }^{17}$

- And, because there is no central administrator in the system or any TOS that users have to accept, contracts are also not essential to governing how people use Bitcoin as a means of payment. $^{18}$

So we can conclude that, owing to conceptual reasons, the central factor in Bitcoin's governance structure is code. The primary way to govern user behavior and tackle practical challenges resulting from its conceptual shortcomings, therefore, is to adjust the code of Bitcoin.

The introduction of "multi-signature" transactions, through which an automated arbitration mechanism can be realized in the Bitcoin architecture, represents a prominent and interesting case of such an adjustment by a change of code. Bitcoin is a complex, decentralized technological system offering advanced network-based services that was able to adapt to a conceptual challenge through activating its own governance structure, and in the process creating a structure for resolving conflicts with deals and transactions.

With these reflections on the mission and function of the change of Bitcoin's architecture in mind, we will now change our perspective to focus on the governance structure of Bitcoin, which enabled this change to take place.

\section{Organizational Model and Structure}

\section{A. Overarching Structure}

In order to grasp how this architectural change was implemented, we must take a closer look at how adjustments in the Bitcoin ecosystem can be accomplished in general. We have seen that Bitcoin's primary mode of influencing behavior is through code, so the leading questions are: Which actors make up the governance group that decides on changes of the code? And what is the internal structure of the group?

As described above, we adopt a broader definition of "code" than simply source code. That said, hardware is of no importance to the arbitration functionality in Bitcoin's system code; the new features are enabled purely by software. Thus, we can narrow our focus to the actors involved in the development of the software used by Bitcoin users.

While there are several Bitcoin clients that provide end users with "wallets" on different operating systems and devices, ${ }^{19}$ the Bitcoin core client ("original client") is the central software in the currency's ecosystem. Since nearly all crucial functionalities of the network are provided

\footnotetext{
${ }^{17}$ Certainly there are general social norms on how to process business deals and there are also special social norms concerning the "right" ways of using Bitcoin evolving in its user community. Both of these affect user behavior, but the concept of Bitcoin does not rely on these elements to fulfill a central function in its system. $C f$. https://bitcoin.org/en/developer-guide\#contracts (retrieved September 10, 2014).

${ }^{18}$ We have to distinguish these from the contracts that are concluded between Bitcoin users to process the underlying business deals. These business contracts include reciprocal provisions for user behavior, e.g. that a user has to initiate the transaction of bitcoins when he received the good he purchased. But the original concept of Bitcoin did not cover these contracts, which meant that enforcement mechanisms in case of a breach have been missing.

${ }^{19} C f$. https://bitcoin.org/en/choose-your-wallet (retrieved September 10, 2014).
} 
and processed by machines running the core client, other clients have to be designed in compliance with the core client's source code. ${ }^{20}$ For that reason, the original client forms not only the software backbone of the network, but in a sense also the blueprint for other implementations: all other clients are descendants from that original client. Whoever wants to change Bitcoin's code must change the code of the original client. We can therefore focus on the governance group around the Bitcoin core client.

As mentioned above, the Bitcoin core client is open-source software. ${ }^{21}$ Simply put, this means software whose source code is open and accessible to the public, mostly via special online platforms like GitHub, sourcefourge.org, or code.google.com. By providing the technological means to organize open-source software development, these platforms form a dominantly codebased governance structure for that process. They can be understood as a meta-structure influencing the governance group of the Bitcoin core client and its processes of participation.

\section{B. Participation}

\section{Primary Categories of Participation}

Open source software often comes with certain inherent expectations regarding participation. ${ }^{22}$ Someone wishing to launch a software project on any open-source platform starts by uploading and publicly hosting the initial source code on a repository. Other developers can then subscribe to that repository and submit new source code as suggestions for improvement. The developer in charge of a project's code repository has full control over the changes to the source code because she can decide which suggestions are accepted as official modifications. Sometimes, a group of users manages the repository instead of an individual.

Additionally, these platforms provide revision control features, which track every change in the source code so that they can be reverted, if necessary. In fact, these version control systems are central tools in (collaborative) software development in general, in open- source and proprietary contexts alike. The technical interaction between these platforms and their users is strongly influenced by the use of revision control software. The platforms provide teams with a central storage for the source code while publishing it at the same time. Some platforms also offer social networking functions that enable users to stay up-to-date and discuss and review changes to the code. In this way, these platforms help manage projects and organize the collaborative workflow and, in a sense, determine the primary categories of participation.

Bitcoin's main client is hosted on GitHub, ${ }^{23}$ a popular open-source platform. All changes to Bitcoin's source code are organized through GitHub. The (social) interaction on that platform is

\footnotetext{
${ }^{20}$ Alternative nodes must "follow the reference client $100 \%$ (bug for bug)," https://en.bitcoin.it/wiki/Category:Nodes (retrieved September 10, 2014).

${ }^{21} \mathrm{Cf}$. https://bitcoin.org/en/developer-documentation and http://opensource.org/licenses/mit-license.php (retrieved September 10, 2014).

${ }^{22}$ Cf. http://opensource.org/docs/osd (retrieved September 10, 2014). The approaches may differ among the several software projects more or less, but it is beyond the scope of this paper to discuss those similarities and slight variances within the open-source scene. Many statements in this section might be characteristic for open-source software and others not. Our goal is only to conceive an idea-how the distributed governance group behind Bitcoin is formed - while having in mind that it is a distinctive example of open-source programming.

${ }^{23} \mathrm{https}$ ://github.com/bitcoin/bitcoin (retrieved September 10, 2014). The source code of the website "bitcoin.org" is also stored and maintained via GitHub.
} 
where the refinement of the code can best be observed and is therefore a good starting point to examine the governance group behind Bitcoin.

On GitHub, registered users can work on the code repositories that belong to their accounts and make use of the described features like version control. Users can also work on the source code together with others by adding collaborators to a repository. Furthermore, GitHub offers its users the ability to start and join organizations, which are the "owners" of the project's code repositories. Members of an organization can be structured in teams and granted different levels of permission:

- Administrators can add new or remove members of the organization.

- Ordinary members may revise the code in the repositories. ${ }^{24}$

- Users who are not part of the organization can submit code proposals. ${ }^{25}$

Bitcoin is one organization on GitHub. The source code of the core client and several other repositories are managed through this organization structure. In this structure, we can observe three different categories of participation: administrators of Bitcoin's organization on GitHub; members of the organization on GitHub, who consider themselves part of the Bitcoin development team; ${ }^{26}$ and non-members who submit their code proposals to members. This means, in essence, that anyone with the ability to program and the will to participate could be seen as an aspirant for membership of the governance group. Because any person could submit snippets of new source code to the organization, anyone could, in theory, influence the governance structure of Bitcoin.

Nevertheless, in the end, decisions are made — or executed at least — by a team of core developers because only they have the technical permissions to accept submissions. Those core developers form, at least at first sight, Bitcoin's governance group in a narrower sense. Every adjustment to Bitcoin's governance structure must pass through the bottleneck of this small group of people. So let us take a closer look at how this group is composed.

\section{Membership Structure}

According to the Bitcoin page on GitHub, 247 people have contributed to the original client, ${ }^{27}$ but only seven members in the organization are currently listed as core developers. ${ }^{28}$ As has been mentioned above, a contributor only has to create an account on the GitHub platform to be able to submit code proposals for the core client or to make use of social networking features like commenting on others' changes to the code. Becoming a member of an organization is not quite as simple: the owner or an administrator of the organization must add the user.

\footnotetext{
${ }^{24} \mathrm{https}$ //help.github.com/articles/permission-levels-for-an-organization-repository (retrieved September 10, 2014). So the main task of an organization's members is to work on the development of the code in their repositories or to decide whether code proposals of other GitHub users become part of the project or not.

${ }^{25}$ This procedure is called "submitting a pull request," because the maintainer of the source code is supposed to "pull" the proposals into the current development line.

${ }^{26} \mathrm{https}$ //github.com/bitcoin/bitcoin (retrieved September 10, 2014).

${ }^{27} \mathrm{https}$ ://github.com/bitcoin/bitcoin (retrieved September 10, 2014); https://bitcoin.org/en/development (retrieved September 10, 2014).

${ }^{28}$ https://github.com/orgs/bitcoin/people (retrieved September 10, 2014).
} 
It is not clear who exactly has this central membership authority in the case of Bitcoin. Gavin Andresen, Chief Scientist at the Bitcoin Foundation, is listed as "Project Lead" on several Bitcoin-related Internet pages. ${ }^{29}$ But we can only guess at the actual roles, teams, and competencies of the other Bitcoin core developers because we could not locate additional publicly accessible information. ${ }^{30}$

Nor we did find any evidence for institutionalized rules of entry or exit laid down for this governance group. One of the core developers was cited on a news page about Bitcoin calling the development team a "meritocracy." " This metaphor is an interesting self-description: it points to the fact that people are judged by their merits in the Bitcoin community. It seems fair to assume, then, that current core developers ask an ordinary member to join the core team if this user has made numerous valuable contributions over a certain period of time.

The metaphor of a meritocracy also helps us understand how the group determines what code submissions are approved. As noted above, members must approve code submissions. Generally, developers copy the whole code into their own personal accounts, work on it, and finally submit a request for the organization to add the code proposal into the repository. The request appears on Bitcoin's GitHub development page so that anyone can review and comment on it. Eventually, another core developer closes the request or pulls it into the development code line. Our analysis of recent code changes to the Bitcoin client shows that trivial code proposals (i.e., small bug-fixes) are pulled into the code without further discussion. In contrast, non-trivial changes must achieve consensus before they are adopted. ${ }^{32}$ Core developers give themselves leeway regarding the question of what is trivial and what is not. Core developers submit their own code changes using the same processes of participation; in many cases the core developers open their own proposals to public debate before pulling them into the main development line.

\section{Mechanisms for Participation}

The most common avenue for participation is revising the source code, as described above. As we have seen, both ordinary and core contributors can propose new source code, which will either be accepted or rejected based on public discussion.

In addition, there is a mechanism of participation for proposals of new features on a conceptual level: the "Bitcoin Improvement Proposal" (BIP). BIPs are essentially concept papers on potential new functionalities in the Bitcoin software. In most cases these new functionalities require more sophisticated changes or additions to the source code than smaller features or bug fixes. Therefore, a BIP consists of technical details about the proposed feature and different kinds of meta-information like the author's name and the status of the document. All submitted BIPs are listed in a repository on GitHub as well as on the Bitcoin Foundation's wiki site. ${ }^{33}$

\footnotetext{
${ }^{29} C f$. https://bitcointalk.org/index.php?topic=7269.0 (retrieved September 10, 2014).

${ }^{30}$ To unveil the distribution of power in this Governance Group would need further research based on methods like discourse analysis or network analysis.

${ }^{31} \mathrm{http}$ //www.coindesk.com/bitcoin-developer-jeff-garzik-on-satoshi-nakamoto-and-the-future-of-bitcoin/ (retrieved September 10, 2014).

32 https:/github.com/bitcoin/bitcoin/blob/master/README.md (retrieved September 10, 2014).

${ }^{33} \mathrm{https} / /$ github.com/bitcoin/bips/blob/master/README.mediawiki (retrieved September 10, 2014);

https://en.bitcoin.it/wiki/Bitcoin_Improvement_Proposals (retrieved September 10, 2014).
} 
If a developer wants to add a new feature to the Bitcoin software, she can author a specification draft. This document must then be sent to the developer mailing list, to which the development community subscribes. After discussion on the mailing list, the draft is added to the list of BIPs if a majority accepts the listing of the proposal. BIPs thus allow substantive debate on the changes in the protocol even before developers put any effort into programming source code. Furthermore, people without the will or the ability to program are able to take part in the discussion on the further evolution of Bitcoin. ${ }^{34}$ If the BIP reaches consensus in the broader public debate on GitHub, a core developer will set its status to "active," 35 and a code contributor can start to write an implementation. This implementation draft can then be submitted as an ordinary source code pull request using the participation process described above.

Jeff Garzik, a core developer of Bitcoin, made an insightful remark about the dynamics of the two mechanisms of participation. He told a news website that the Bitcoin community considered itself very conservative when it comes to new functionalities. The core developers generally did not accept completely new features if these were made as concrete source code proposals. Instead the core development team would always seek to reach a high level of consensus among the Bitcoin community about new functionality. ${ }^{36}$ Additionally, the BIPs function as a mechanism for slowing down the process of change.

The development of multi-signature transactions began in the form of two different BIPs. ${ }^{37}$ This is not surprising given that multi-signature transactions were a major update to Bitcoin's functionalities. Reviewing the discussions around those BIPs in the mailing list archives, we found several threads already discussing the details of these proposals. ${ }^{38}$ Interestingly, a general debate on the pros and cons of this new functionality never came up, at least as far as we could see. A possible explanation for this is that the community had already recognized the necessity of such a feature. In the end, the BIP process helped to confirm consensus on the functionality and establish consensus on side issues of how to implement the arbitration mechanism in the Bitcoin system.

\section{E. Decision-Making Structures}

\section{Decision Makers}

\footnotetext{
${ }^{34}$ Aside from GitHub's communication features, a large online community interested in Bitcoin and its development utilizes various common online communication tools like wikis, forums, IRC-chats or mailing lists. Debates on new features could therefore also take place with the involvement of other stakeholders in the Bitcoin ecosystem like the miners, the users, etc.

${ }^{35}$ Cf. supra note 34 .

${ }^{36}$ Bradbury, Danny (2013): Bitcoin developer Jeff Garzik on Satoshi Nakamoto and the future of Bitcoin, http:/www.coindesk.com/bitcoin-developer-jeff-garzik-on-satoshi-nakamoto-and-the-future-of-bitcoin/ (retrieved September 10, 2014).

${ }^{37}$ https://github.com/bitcoin/bips/blob/master/bip-0010.mediawiki (retrieved September 10, 2014); https://github.com/bitcoin/bips/blob/master/bip-0011.mediawiki(retrieved September 10, 2014).

${ }^{38}$ Cf. http://sourceforge.net/p/bitcoin/mailman/message/28374763/ (retrieved September 10, 2014); http://sourceforge.net/p/bitcoin/mailman/message/32614927/(retrieved September 10, 2014); http://sourceforge.net/p/bitcoin/mailman/message/27842517/ (retrieved September 10, 2014).
} 
As we have seen in sections $\mathrm{C}$ and $\mathrm{D}$, decisions of core developers are shaped by the social norms of the Bitcoin development community and by the tools that are used to enable community participation and collaborative development. Consensus in the community is a hard guideline for decisions because this benchmark is strictly enforced as a requirement for implementation of proposals.

It is important to note that the overarching goal among Bitcoin's core developers is to prevent a breakup of the system. Compared with other types of open software projects, this goal is even more fundamental because Bitcoin's success as an alternative means of payment depends on the ubiquity, integrity, and security of the technological infrastructure it provides. It is for this reason that the group is inherently suspicious of new features, and might even reject new features in the face of community support. ${ }^{39}$

To get the entire picture of Bitcoin's governance, we also have to take into account the Bitcoin Foundation. Seven founding members established the Bitcoin Foundation in September 2012. ${ }^{40}$ According to its self-description on its website, the foundation serves three main goals: to foster standardization, to protect the integrity of Bitcoin protocol, and to publicly promote Bitcoin. ${ }^{41}$ What should be noted regarding our case is that the Bitcoin Foundation now functions as an institutional framework for core developers. Lead Bitcoin developer Gavin Andresen is employed by the foundation as "Chief Scientist" and at least three other core developers are also on the foundation's payroll. ${ }^{42}$ This raises the question of whether and how the Foundation might influence Bitcoin's code development.

The founding documents (the Foundation's bylaws) and legal grounding (legal provision by District of Columbia's law) of the Foundation delineate its governance structure. Understanding the interaction between the Foundation and the core developers is challenging to answer just by looking at the founding documents of the Bitcoin Foundation. The Foundation's central decisionmaking body is its Board of Directors. ${ }^{43}$ It has seven seats, which are elected by the members of the foundation. There are three different categories of membership: founding members, industry members, and individual members. The founding members mentioned above elect one seat on the board. Companies offering services and products based on Bitcoin can apply to become industry members, and these members have the right to elect three directors. The last three board members are elected by individual members of the foundation. Individual members "shall be natural persons transacting in, promoting or otherwise contributing to the Bitcoin system or other similar distribute-digital currency system."44 Individual membership thus is the way for the Bitcoin user community to be represented in the Foundation.

Since there is no public record of the Foundation's decisions, we did not find any evidence that the Board of Directors is influencing the strategic decisions of the core developer team. Yet we

\footnotetext{
${ }^{39}$ Cf. Bradbury (2013), supra note 37: “This notion of consensus doesn't necessarily make Bitcoin a push-button democracy, though; the core developers carry ultimate veto, and they're notoriously cautious."

${ }^{40}$ Bylaws of the Bitcoin Foundation, Sec. 3.2. https://github.com/pmlaw/The-Bitcoin-Foundation-Legal-

Repo/blob/master/Bylaws/Bylaws_of_The_Bitcoin_Foundation.md (retrieved September 10, 2014).

${ }^{41} \mathrm{https}$ ///bitcoinfoundation.org/about/overview/ (retrieved September 10, 2014).

${ }^{42} C f$. https://bitcoinfoundation.org/about/overview/ (retrieved September 10, 2014).

${ }^{43}$ Cf. Bylaws of the Bitcoin Foundation, Sec. 5.

${ }^{44}$ Bylaws of the Bitcoin Foundation, Sec. 3.2.
} 
assume that there is at least some kind of coordination of decisions because the interests of the industry members are affected by strategic decisions on the technological evolution of Bitcoin. ${ }^{45}$ As a core developer, as well as a member of the Foundation's Board of Directors, Gavin Andersen could act as link between them.

Another interesting observation is that the Bitcoin Foundation represents an institutionalized central administrator in a system originally designed as a decentralized network. ${ }^{46}$ If additional research were to show that the Bitcoin Foundation is in fact powerful in shaping the evolution of the system, we would have a good reason to ask how the system handles the resulting frictions between conceptual expectations and factual reality.

\section{Outcomes}

\section{A. Resolution of Problem}

The introduction of multi-signature transactions demonstrates the application of Bitcoin's predominantly code-based governance structure. Our analysis of that case showed that the decision-making processes regarding transformations of Bitcoin's architecture are not as transparent as one might expect given the cryptocurrency's commitment to open source. Major changes to the code are publicly discussed in the Bitcoin developers' community and we could not find a single case of a decision in which the core development team deviated from the consensus of the community. But the true nature and extent of the direct and indirect influence of the Bitcoin Foundation and its bodies on current processes is largely unclear.

It is useful to distinguish between two levels of governance structures in online services. On the first level there is the normative background of user behavior and the second level is a metastructure that frames the processes of structural evolution on the first level (see fig. 2 below).

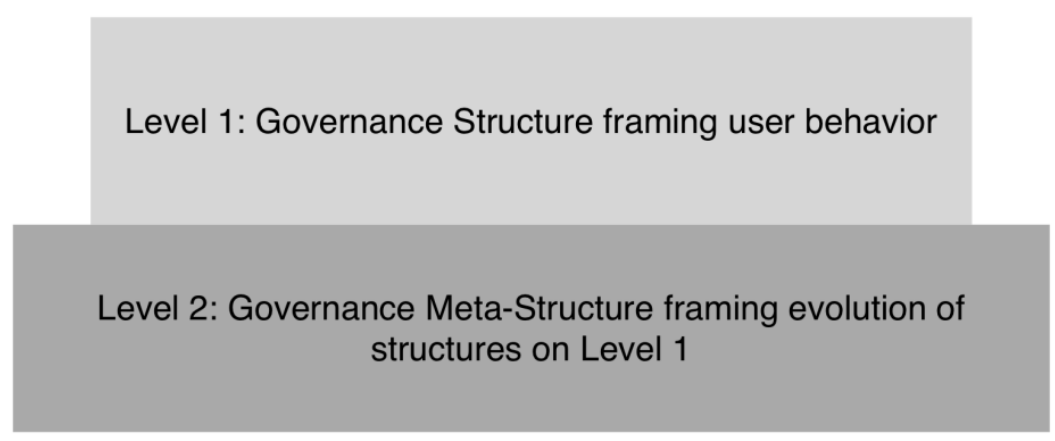

Figure 2.

On an abstract level, we can take the case of Bitcoin's arbitration mechanism as an example of the implementation of a governance (infra)structure that tackles a complex social challenge by offering a means for dispute resolution (level 1). As we have seen, that evolution is shaped by social norms and the code of the platforms on which Bitcoin's source code is maintained and further developed (level 2). Together, these factors form a complex multi-layer structure that

\footnotetext{
${ }^{45}$ To prove this assumption we would need to use additional methods like participating observation or expert interviews.

${ }^{46} C f$. Bylaws of the Bitcoin Foundation, Sec. 1.1 and Sec. 2.2.
} 
defines and enables Bitcoin as a payment system. In this regard, Bitcoin might be comparable to other complex open-source technological systems like Linux or Wikipedia. An interesting follow up to this paper would be to analyze whether these projects also have an underlying multi-level governance structure.

\section{B. Best Practices and Templates}

The first lesson that we can take away from this case study is that the evolution of governance in open-source online services can be highly dynamic because its underlying code can be changed more easily than, for example, law.

Furthermore, we have seen a case of a decentralized, primarily code-based governance structure (level 2) that was invoked in order to create a code-based governance structure for addressing disputes between users (level 1). It is important to recognize the interactions between the two levels. A first hypothesis derived from this analysis is that an open, code-based governance structure serving such a highly complex social function as providing the normative foundation for a payment system is itself in need of meta-structures on a second level that frame the evolutionary processes of the structures on the first level.

At this point, it is important to clarify that we are not making the argument that political and normative questions are not of importance in such systems that at first glance might seem decentralized. On the contrary, they become even more relevant. In fact, if the normative structures become multi-layered, and more and more complex, questions about power and legitimacy only become more important.

Finally, this case evokes the question of whether code-based dispute resolution systems could be a model for dealing with conflicts in other systems. In terms of governance structures this is certainly possible. Further contemplation of this possibility leads us back to the question on how the meta-structures framing the development processes would have to be configured in these systems to achieve this aim in a transparent and legitimate way. The concept of open source could be a starting point but is not the last word on this question, as our case study has shown. ${ }^{47}$ From our perspective, this aspect needs more research. Additionally, analysis of a second evolutionary process for Bitcoin's architecture would help corroborate our findings and assist in determining whether they are generalizable at least within Bitcoin's system. ${ }^{48}$

\footnotetext{
${ }^{47}$ As far as public interest would be affected by conflicts in this system, rationality of decisions would have to be guaranteed by procedural rules and sets of pertinent criteria.

${ }^{48}$ A promising second case is already in sight: the Bitcoin community is currently discussing whether a deep code change is needed in response to a certain mining cooperative providing for a short period of time over $50 \%$ of the system's overall computing power. During this time, the mining group was theoretically able to control all the transactions in Bitcoin. Because antitrust laws are not enforceable, this situation fundamentally challenges the system in a comparable manner to the case of the missing infrastructures for arbitration. $C f$. http:/hackingdistributed.com/p/2014/06/13/in-ghash-bitcoin-trusts/ (retrieved September 10, 2014).
} 


\section{Appendix C: Creative Commons (CC)}




\title{
NoC Internet Governance Case Studies Series: Creative Commons (CC)
}

\author{
Federico Morando \\ Nexa Center for Internet \& Society at Politecino di Torino
}

\begin{abstract}
Editorial Note: Context, Character, and Purpose of the Case Study
This case study is part of a globally coordinated, independent academic research pilot project by the Global Network of Interdisciplinary Internet \& Society Research Centers (NoC). Facilitated by the Berkman Center for Internet \& Society at Harvard University, this study examines existing multistakeholder governance groups with the goal of informing the future evolution of the Internet governance ecosystem. Building upon the NETmundial Principles and Roadmap, it contributes to current policy debates at the international level, including the Internet Governance Forum, the NETmundial Initiative, and other organizations and efforts.
\end{abstract}

Internet governance is an increasingly complex concept that operates at multiple levels and in different dimensions, making it necessary to have a better understanding of both how multistakeholder governance groups operate and how they best achieve their goals. With this need in mind, at a point where the future of Internet governance is being re-envisioned, colleagues from several NoC institutions around the world have written twelve case studies examining a geographically and topically diverse set of local, national, and international governance models, components, and mechanisms from within and outside of the sphere of Internet governance. Key findings from these cases are summarized in a synthesis paper, which aims to deepen our understanding of the formation, operation, and critical success factors of governance groups and even challenge conventional thinking.

The research, based on twelve case studies, suggests that there is no single best-fit model for multistakeholder governance groups that can be applied in all situations. Rather, it reveals a range of approaches, mechanisms, and tools available for both the formation and operation of such groups. The analysis demonstrates that the success of governance groups depends to a large degree on the careful selection, deployment, and management of suitable instruments from this "toolbox." As governance groups pass through different phases of operation, conveners and facilitators must remain alert to changes in circumstances that necessitate adjustments to the approaches, mechanisms, and tools that they deploy in order to address evolving challenges from inside and outside. This case study provides insights into how those instruments can be deployed and adjusted over time within such groups, and highlights how their interactions with important contextual factors may be successfully managed within given resource restraints.

The research effort is grounded in a diversity of global perspectives and collaborative research techniques. Adhering to objective and independent academic standards, it aspires to be useful, actionable, and timely for policymakers and stakeholders. More broadly, the Network of Centers seeks to contribute to a more generalized vision and longer-term strategy for academia regarding its roles in research, facilitation and convening, and education in and communication about the Internet age.

For additional information on the initiative, please contact Urs Gasser, Berkman Center for Internet \& Society, at ugasser@cyber.law.harvard.edu. 


\begin{abstract}
This case study examines the formal structure of Creative Commons (CC) as an organization stewarding the development of the most well-known and widespread set of standard public copyright licenses. In particular, the case study explores the interaction of $\mathrm{CC}$ with its own community and with a broader set of international stakeholders during the drafting of the fourth version of the Creative Commons Public Licenses, which took place between mid 2011 (with public discussion starting in November 2011) and November 2013. This case study highlights how a transnational organization can operate outside of the scope of formal regulatory authority, but with the purpose of creating an output (CC licenses) that have legal weight. In such an environment credibility and legitimacy are paramount, necessitating opportunities for public consultation, transparency, and even admitting mistakes.
\end{abstract}




\section{Table of Contents}

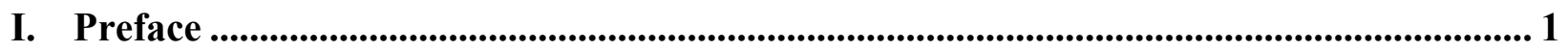

II. Introduction .................................................................................................................................... 1

III. Participation ............................................................................................................................... 3

A. The Affiliate Network.............................................................................................................. 3

B. License Users............................................................................................................................ 4

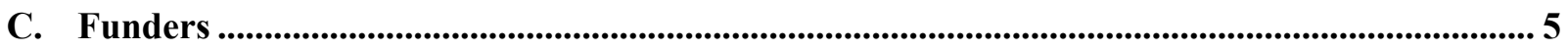

IV. Organizational Model and Structure ...................................................................................... 6

A. The Governance of Creative Commons Corporation ......................................................................... 6

1. The Professionalization of Creative Commons Corporation ………............................................ 8

B. The Governance of the Licensing Process........................................................................................ 9

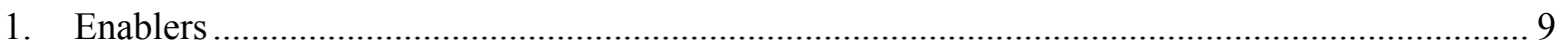

2. Interaction Between Technical and Non-Technical Stakeholders ............................................ 10

3. Interaction Between Geographic Spheres ......................................................................... 11

C. Final Decision-Making Process......................................................................................................... 13

1. The Role of Documentation and Institutional Memory …….................................................... 14

2. Conflict Management and Time ....................................................................................... 14

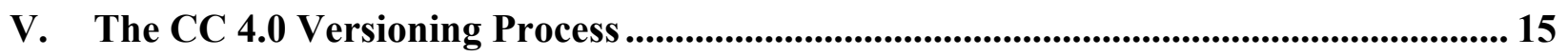

A. Background and Brief History of the 4.0 CC PLs..................................................................... 15

B. The Governance of the 4.0 Versioning Process.................................................................................... 17

1. Final Decisions on the Most Heated 4.0 Drafting Issues ........................................................ 18

VI. Conclusions and Lessons Learned............................................................................................. 19 


\section{Preface}

This case study examines: a) the formal structure of Creative Commons (CC) as an organization stewarding the development of the most well-known and widespread set of standard public copyright licenses, and b) the interaction of $\mathrm{CC}$ with its own community and with a broader set of international stakeholders. The case study focuses on the drafting of the fourth version of the Creative Commons Public Licenses, which took place between mid-2011 (with public discussion starting in November 2011) and November 2013 (when the licenses were finalized and published).

This draft case study is based on desk research, written interviews with two senior members of the management of Creative Commons Corporation-Diane Peters, General Counsel and Corporate Secretary, and Timothy Vollmer, Public Policy Manager-, and oral and written interviews with members of the Creative Commons Affiliate Network. Interviews were conducted between July and September 2014. The author of this case study has also been directly involved in the activities of the Creative Commons Affiliate Network since 2006 and, since 2012, has led Creative Commons Italia, the Italian working group affiliated with the CC Network.

\section{Introduction}

"Creative Commons" is an evocative brand, but this label may point to various and distinct (although tightly interlinked) entities. The Creative Commons Corporation (CCC) is a US-based non-profit organization incorporated under the law of Massachusetts. ${ }^{1} \mathrm{CC}$ is also a community of people and organizations more or less loosely joined. At the core of this community is the Creative Commons Affiliate Network, which consists of more than 100 affiliate institutions in over 70 jurisdictions around the world. Each affiliate is, in turn, a more or less formal organization providing the focal point for a local community of volunteers. The Affiliate Network, together with other volunteers, participates in the CC mailing lists and online tools (e.g., wikis) where issues are discussed and information exchanged, and in various regional or thematic meetings. Since 2011, CCC has also systematically organized yearly Global Summits where the CC community may meet physically. Similar meetings were also organized prior to 2011, but on a less regular basis, usually in connection with other events and conferences.

The main tool and foremost output of Creative Commons (both the organization and the larger community) is the Creative Commons Public Licenses (CC PLs, or simply CC licenses), which are designed to further CC's mission.

This is how the official Creative Commons website describes CC: "Creative Commons is a nonprofit organization that enables the sharing and use of creativity and knowledge through free legal tools. Our free, easy-to-use copyright licenses provide a simple, standardized way to give the public permission to share and use your creative work - on conditions of your choice. CC licenses let you easily change your copyright terms from the default of "all rights reserved" to

\footnotetext{
${ }^{1} \mathrm{CCC}$ used to be headquartered in Mountain View, California, but in October 2014 it went (even more) virtual, closing its physical office. Currently, CCC staff members are spread across six US States and two Canadian provinces, without counting regional coordinators all over the world. See https://creativecommons.org/weblog/entry/43873.
} 
"some rights reserved." Creative Commons licenses are not an alternative to copyright. They work alongside copyright and enable you to modify your copyright terms to best suit your needs."

The CC Affiliate Network shares the mission of the CCC: "Creative Commons develops, supports, and stewards legal and technical infrastructure that maximizes digital creativity, sharing, and innovation."

The vision of the CCC is arguably also the cohesive goal of the CC as a community of creators, enthusiasts, and activists: "Our vision is nothing less than realizing the full potential of the Internet - universal access to research and education, full participation in culture - to drive a new era of development, growth, and productivity."

In practice, CC's goals are pursued through developing a set of copyright licenses and tools aimed at exercising copyright and related rights in a flexible and overall permissive way - which is consistent, in particular, with the opportunities of circulation and remixes offered by digital technologies. The common trait of the $\mathrm{CC}$ legal tools is to provide simple, standardized, and modular ways to allow a certain selection of uses and re-uses (i.e., edits, remixes) of creative works. CC enables all of this without requiring creators to possess a highly sophisticated legal background, without requiring any fees, and while allowing users and re-users (i.e., creators of derivative works) to benefit from the reduced transaction costs and higher interoperability created by a set of standardized legal tools.

\section{The Creative Commons Public Licenses (CC PLs)}

Creative Commons users (i.e., the Licensors) may select the license to apply to their creative works (or databases) by answering two simple questions: "Allow adaptations of your work to be shared?" (Answer options: "Yes," "No," or "Yes, as long as others share alike"); "Allow commercial uses of your work?" (Answer options: "Yes" or "No").

The six potential combinations of answers generate the different Creative Commons Licenses, which are built on the basis of four standard modules:

- Attribution (BY): the common standard module, requiring that the author is credited for the original creation;

- ShareAlike (SA): licenses including the SA element require that new creations building on the original material are licensed under identical terms. Under this "copyleft" or "viral" approach, the license of the original work "infects" derivative works downstream;

- NoDerivatives (ND): licenses including the ND clause just allow for verbatim reproductions. Licensees may pass along the work, but it must not be changed in a manner that constitutes a derivative work;

- NonCommercial (NC): the NC clause forbids the use of the material for commercial purposes (i.e., uses primarily intended for commercial advantage or monetary compensation).

\section{Figure 1.}


As a complement to (or catalyst for) its legal tools, Creative Commons also: (a) raises awareness of copyright law and of the benefits associated with permissive copyright licenses; (b) produces informational and educational materials (standard FAQs, videos, etc.); (c) strives to standardize machine readable metadata concerning licensing (providing its own standard solution, also used by several search engines in their advanced features); and (d) advocates for both free and open knowledge and copyright reform.

This case study focuses on the governance of Creative Commons as an organization, a community, and a standards setting organization, interacting with a broad range of stakeholders to produce, maintain, and develop the aforementioned set of legal tools, which represent a relevant part of the legal infrastructure of the Internet. (As an example, Creative Commons licenses are used by Wikipedia as the legal core of its "social contract," complementary to its mission as an online encyclopedia that can be edited by almost anyone.)

\section{Participation}

The Creative Commons ecosystem includes individuals, who choose to use CC licenses, as well as major media organizations, online service providers, academic organizations, and government players. All these stakeholders explicitly influence the governance of CC. Funders are another stakeholder, but the Creative Commons Corporation is committed to remaining independent of funder-driven outcomes, particularly in terms of their impact on the governance of the $\mathrm{CC}$ community.

\section{A. The Affiliate Network}

The Creative Commons Affiliate Network, which consists of more than 100 affiliate institutions in over 70 jurisdictions around the world, represents the inner circle of the Creative Commons community. ${ }^{2}$ The Affiliate Network is a web of diverse organizations, where each affiliate institution is, in turn, the hub of a local community of volunteers.

Typical affiliates are universities (or their libraries) or research centers, mostly with a focus on law or ICT/technology, and other non-profit organizations, mostly associations/foundations working to promote open knowledge, increase digital literacy and education, support free/open source software, or foster digital rights/freedoms. These include some national chapters of Wikimedia and Open Knowledge (formerly the Open Knowledge Foundation). Some affiliate institutions are rather informal or specialized, e.g., hacker spaces or similar meeting spaces in between cultural organizations and co-working facilities.

The CC Affiliate Network is open to new members even in jurisdictions where an affiliate institution already exists. Creative Commons does screen new applicants to the Affiliate Network to ensure adequate resources, and alignment of vision and goals. Upon completion of the screening process, each new affiliate signs a formal affiliate agreement with the Creative Commons Corporation. If an applicant seeks to join the Affiliate Network in a jurisdiction where there is an existing affiliate, then under the terms of the affiliate agreement, Creative Commons must first consult with that existing affiliate(s) before any decision is made.

\footnotetext{
${ }^{2}$ See https://wiki.creativecommons.org/CC_Affiliate_Network for additional information, including the full list of affiliate institutions.
} 
Affiliate members (i.e., individuals with an affiliation within the Affiliate Network) constitute the backbone of the Creative Commons community, and there is a dedicated and private mailing list for the Affiliate Network, where some discussions take place exclusively. That said, most decision-making processes concerning Creative Commons also involve public mailing lists, where there are virtually no barriers to entry. Therefore, any interested person may have a say within the Creative Commons community. That said, affiliate members typically receive previews of internal drafts, longer comment periods, and other privileges that serve to make their voice more relevant and impactful.

\section{B. License Users}

According to the State of the Commons Report, ${ }^{3}$ Creative Commons users (i.e., licensors adopting the CC PLs) number in the order of millions. In November 2014, according to statistics derived from Google data, there were over 882 million pieces of CC-licensed content on the Web (they were about 400 million in 2010, 50 million in 2006).

Some of the best-known users of Creative Commons licenses are the following:

- Flickr was one of the first major online communities to incorporate Creative Commons licensing options into its user interface. Currently more than 306 million pictures are available under a CC license.

- Google enabled CC-search capabilities through its main search engine, image search engine, and book search engine, and Google allows users to CC license their own content in Picasa, YouTube, and other applications.

- MIT OpenCourseWare has used CC licenses since 2004 and has over 1900 courses available freely online for anyone, including the right to adapt, translate, and redistribute the content.

- The Public Library of Science's Open Access journals - such as PLoS Biology, PLoS Medicine, and PLoS ONE-are well-known and respected in scholarly communication.

- Wikipedia, the largest and most cited collaborative free online encyclopedia, uses the Creative Commons Attribution ShareAlike license as its copyright license.

- The Obama Administration uses CC licenses in various ways, from licensing presidential campaign photos to using the CC Attribution license as the default choice for third-party content posted on Whitehouse.gov (content produced by the administration is automatically in the public domain).

In some cases, Creative Commons interacts directly with major institutions and/or communities that either release a large volume of content under CC licenses, release content with significant cultural or educational relevance, or carry a certain level of prestige. The following are two examples in which Creative Commons interacted with major users in ways that led to significant changes in the $\mathrm{CC}$ licenses and/or to significant institutional arrangements.

MIT's OpenCourseWare project was launched prior to the formal release of the Creative Commons licensing suite in December 2002. Since then, OpenCourseWare has used an early version of the Attribution-Non-Commercial-Share Alike license. Given its extraordinary worldwide reputation, MIT wanted to ensure that translations and adaptations of its learning material were properly described as such, without implying any endorsement of or direct

\footnotetext{
${ }^{3}$ https://stateof.creativecommons.org/report/ (accessed on Dec. 4, 2014).
} 
relationship with MIT. To address such concerns, a "No Endorsement" clause was explicitly added to the 3.0 version of $\mathrm{CC}$ licenses. In this case, most of the $\mathrm{CC}$ community and legal experts agree about the fact that the clause was likely not strictly necessary from the legal point of view (at least in most jurisdictions), but there were similar "No Endorsement" clauses in several free and open source software licenses. Moreover, such a clause would certainly not hurt the overall legal robustness of the license and, as CC put it, it was also useful "to give the licensor comfort and to ensure that the licensee was under no misapprehensions." ${ }^{4}$

Until 2009, Wikipedia licensed its content with the GNU Free Documentation License (or GNU FDL), a "copyleft" license for software documentation designed by the Free Software Foundation (FSF) for its GNU Project. GNU FDL was one of best standard licensing options available for encyclopedic content when Wikipedia was launched in 2001, but over time it became apparent that GNU FDL was less user-friendly and less widespread (in terms of licensing non-software material, such as ordinary texts and pictures) than the Creative Commons licenses. In December 2007, Wikipedia founder Jimmy Wales announced that, after a long negotiation, the Free Software Foundation, Creative Commons, and the Wikimedia Foundation had produced a proposal to modify GNU FDL to allow the possibility for the Wikimedia projects (including Wikipedia) to migrate to the CC Attribution Share-Alike license. ${ }^{5}$

When asked about the lessons learned from their experiences with these various stakeholder communities, interviewed CCC staff members stated that CC learned a lot about transparency and consensus-based decision making from the Wikimedia Foundation, Open Knowledge, and the Free Software Foundation. This process is also an example of how these organizations reciprocate with and learn from one another. For example, the Creative Commons Affiliate Agreement (last overhauled in 2011-2012) served as a benchmark for the chapter agreements of both Open Knowledge and Wikimedia.

Governments are another class of major users and relevant stakeholders in the Creative Commons ecosystem. An example of interaction with governments will be discussed below, in describing the background and brief history of the 4.0 CC PLs.

\section{Funders}

Finally, Creative Commons has mechanisms in place (such as a conflict of interest policy for Board and staff members) to ensure that funders have minimal influence in the governance of CCC. ${ }^{6}$ According to our interviews, affiliates seem to share the impression that the influence of

\footnotetext{
${ }^{4}$ Quoted from https://wiki.creativecommons.org/Version_3\#MIT.

${ }^{5}$ For completeness: Creative Commons' initial work had focused on achieving compatibility between the CC licenses, and in particular the 3.0 version, and the GNU FDL. Such negotiation failed, however the FSF agreed to implement some changes in the 1.3 version of the GNU FDL, including a time-limited provision allowing certain materials (produced on "Massive Multiauthor Collaboration Sites") to transition to a Creative Commons Attribution Share-Alike license without obtaining the permission of every author, which would ordinarily be necessary. ${ }^{6}$ Another potentially relevant policy of CCC is the following: A condition of receiving grants is that the output of the activities performed be made available under a $\mathrm{CC}$ license (and cannot be owned by the funder) - as CCC staff members put it, this is an "indirect way [that] ensures that foundations abide by our policy of making publicly-funded works freely available."
} 
funders in the governance of CC is limited and comparatively much smaller than that of actual or potential licensors.

With respect to the perception of the general public, some scant criticism on blogs and mailing lists makes reference to specific corporate interests (e.g., Google), but there is no systematic evidence of criticism of CC on the basis of its relationship with funders (for instance, this aspect is not even touched upon in http://en.wikipedia.org/wiki/Creative_Commons\#Criticism).

On the other hand, there is an objective lack of geographic diversity in the funding of CCC primarily driven by its incorporation in the United States, relevant tax laws, and the unique role of philanthropic giving in the U.S. This may contribute to the fact that CCC is still perceived as a US organization, despite its global reach and mission.

\section{Organizational Model and Structure}

\section{A. The Governance of Creative Commons Corporation}

Most of the people involved in the Creative Commons community, including most of the active participants in the Affiliate Network, are not aware of the details concerning the governance of Creative Commons Corporation. This is perfectly compatible with an active participation in the community, because the directors and staff of $\mathrm{CCC}$ interact with the rest of the community in largely informal (and at the same time very respectful) ways, giving the impression that the most relevant decisions concerning Creative Commons are made by (rough) consensus emerging from interactions within the $\mathrm{CC}$ community. That said, decision-making power over everything from the legal tools (which are approved, published, and even technically hosted on the servers of $\mathrm{CCC}$ ) to the $\mathrm{CC}$ registered brand ultimately rests with the Creative Commons Corporation, even if many decisions are made using rough consensus.

As described in the Amended and Restated By-Laws of Creative Commons Corporation (as of 30 April 2014), ${ }^{7}$ Creative Commons is a corporation organized under Chapter 180 of the Massachusetts General Laws with no members; CCC's decisions are made by the Directors of the Corporation. By vote the Directors can set the number of Directors to be five or more; it is currently seventeen. The Directors can also elect their successors. In this sense, it seems accurate to describe CCC as governed by an antipoetic elite, whose legitimacy (which appears to be very strong) arguably comes from the extraordinary standing of the Directors (including academics, practitioners, and activists at the highest levels) ${ }^{8}$ in the open knowledge community, and through a track record of successful, careful, and moderate decisions.

The Board of Directors, by majority of the Directors present (including by means of teleconferencing tools) and voting, decides on all material questions affecting the direction of the organization. Some decisions - such as the election of the CEO - may only be taken at the Annual Meeting. Other decisions may be made at other meetings at which there is a quorum of

\footnotetext{
${ }^{7}$ For more information, see the corporate charter (http://ibiblio.org/cccr/docs/articles.pdf) and the by-laws (http://ibiblio.org/cccr/docs/bylaws.pdf).

${ }^{8}$ The current Board consists of 17 members, three of whom are non-voting (individual profiles are available at http://creativecommons.org/board).
} 
one third of the Directors. Some decisions may also be delegated to subsets of the Board, designated as Committees and including an Executive Committee. ${ }^{9}$

The Board currently does not have designated positions available for affiliates or the community, nor does it have a formal policy on appointing regional, community, or affiliate representatives. Instead, quoting CCC staff, "the Board strives to include members who are representative of the community overall." ${ }^{10}$ This is confirmed by the fact that the current Board includes affiliate network members from Africa, Europe, Latin America, and Asia-Pacific. ${ }^{11}$ Thus, while no formal mechanism exists to ensure the participation of specific representatives of the Affiliate Network, or of the CC community, as a practical matter the Board has taken steps to ensure a fair representation by affiliates from various regions. This applies both to the Board itself and its Advisory Council, where all five regions are represented.

Interestingly, in the past, Creative Commons did have a formal process by which two Board members were selected based on recommendations from the Affiliate Network. ${ }^{12}$ The Board no longer actively supports that process and policy. CCC staff as described in the following way the reasons for discontinuing this policy: "In the end, $\mathrm{CC}$ believed it could accomplish the same balance in its board make-up without resorting to formal seats, believing that if seats were reserved that those board members may be considered "representatives" of the particular region or jurisdiction from which they came rather than a representative for all of $\mathrm{CC}$, which was strongly preferred."

Even if the Board of Directors provides strategic guidance to $\mathrm{CCC}$, the day-by-day activities are fully managed by a staff of employees. Currently, CCC employs 16 staff members (plus 9 regional coordinators and 2 consultants). Taking into account salaries, other employee-related costs, and all educational and international activities funded through $\mathrm{CCC}$, the costs in 2011 and 2012 were 2.5 million USD. $^{13}$

\footnotetext{
${ }^{9}$ The Board has established the following committees, which have authority to act in lieu of the full board on certain matters set forth in their respective charters: the Audit Committee, and the Executive Committee. The Board has also established the following additional committee and councils, which do not have authority to act on its behalf but that instead support the Board on matters set forth in their respective charters: the Finance Committee, the Development Council, and the Advisory Council.

${ }^{10}$ This and the following quotations from CCC staff members come from written interviews we conducted during the month of August 2014 with two senior members of the management of Creative Commons. Therefore, in principle, these quotations just represent their personal point of view, and not the point of view of Creative Commons Corporation (which is conveyed by the CC By-Laws and official statements issued by the Board of Directors).

${ }^{11}$ These four areas, plus the Arab world, comprise the five regions into which affiliates are selforganized and in which $\mathrm{CCC}$ has appointed regional coordinators.

${ }^{12}$ See https://wiki.creativecommons.org/Affiliates/Board_recommendation_process, where a structured "Affiliate Recommendation Process for Board Candidates" is described (the process was established and refined between 2009 and 2011).

${ }^{13}$ This figure includes the educational and international activities funded through CCC. See the most recent tax return (http://ibiblio.org/cccr/docs/990.pdf) and most recent audited financial statement (http://ibiblio.org/cccr/docs/audit.pdf).
} 
From the governance point of view, the core CC staff at Creative Commons Headquarters (CC HQ) is comprised of the following people:

- The Chief Executive Officer (CEO) is the president and chief executive officer of the Corporation. The CEO has general supervision and control of the business of the Corporation (subject to oversight by the Directors).

- The Treasurer is the chief financial and accounting officer of the Corporation.

- The Secretary is the Clerk of the Corporation, taking minutes and supporting the Board and its committees and councils in their work.

- The General Counsel is worth an explicit mention amongst the other officers, since the General Counsel is responsible for the management of the organization's general legal affairs. Such affairs are especially relevant for Creative Commons, considering its focus on legal standardization issues.

- The Affiliate Network Coordinator is also worth explicit mention, as the person who is responsible for ensuring that $\mathrm{CC}$ staff and those representing the regions (described below) remain in close contact on matters affecting the affiliates and that affiliate views are represented in staff decisions.

Although not employees, Creative Commons has Regional Coordinators (RCs) responsible for overseeing the efforts of CC's volunteer affiliates around the globe. ${ }^{14}$ At present, there is at least one RC for each of the Middle East, Africa, Europe, Asia-Pacific, and Latin America. The RCs report to the Affiliate Network Coordinator, who is part of the leadership team of Creative Commons.

\section{The Professionalization of Creative Commons Corporation}

In the interviews we conducted within the Affiliate Network, some members of the affiliates also observed that during its more than 10 years of life, the CCC staff has undergone a significant evolution (which is arguably common to many organizations). At the beginning, the CCC staff was described as a group of open knowledge activists and enthusiasts, with good professional skills. Today, it is described as a group of professionals, with a good track record of working with "open" organizations.

When asked if this evolution represented a problem in interacting with CCC, those interviewed mostly agreed that it was not an issue. However, some mentioned moments of friction (sometimes seen as an actual crisis) during the transition, e.g., a tendency to create new and more structured duties for the Affiliates, which mostly remained groups of volunteers. (And, at the same time, the personal ties between the Affiliates and the CC HQ were partly diluted by the arrival of new people.) Such tendencies were, however, moderated by calibrating the new tools - new Memoranda of Understanding, explicit roadmaps, etc - to the needs and possibilities of the various affiliates. At the same time, new personal relationships were established, possibly on a different basis than previously, e.g., more on mutual respect for each other's professional skills than for the fact of being "fellow activists,"

\footnotetext{
${ }^{14}$ See https://wiki.creativecommons.org/Regional_Coordinators.
} 


\section{B. The Governance of the Licensing Process}

Drafting and stewarding licensing tools is at the core of Creative Commons mission. Consistent with the organizational model and legal structure of Creative Commons, ultimate decision making related to legal tools rests with the Creative Commons Corporation (i.e., with the Board of Directors). At the same time, the broader $\mathrm{CC}$ community is extensively consulted both formally and informally (e.g., through informal e-mail discussions or conference calls with subgroups of experts on specific topics). This interaction between the $\mathrm{CCC}$ and the $\mathrm{CC}$ community will be described in more detail below, using the 4.0 versioning process as an example (see page $15)$.

Moreover, all material decisions are publicly documented and explained, with underlying strategic reasons for the decision disclosed. Explanations of differences between all versions are documented on the CC public wiki, with links to announcements and detailed explanations of changes and strategic decisions. ${ }^{15}$ The General Counsel directs this process.

The affiliate members interviewed for this case study confirm both that there is a clear perception of the concentration of formal decision-making power and that the overall process is publicly documented in an accurate way. For instance, a member of the Affiliate Network (and admittedly the most critical person amongst the interviewed people) stated that CC governance uses participative formats and tools, but is, at the same time, "highly centralized and nonegalitarian," in terms of final decision-making power. The interviewed person then added: "Diane [CCC General Counsel] collected very well all the points of view during the consultation process and Sarah [CCC Senior Counsel] and others published regular reviews and updates of the issues, but at the end, Diane took the decisions, which was transparent." When asked why, in such a context, he/she was willing to collaborate, despite the absence of any monetary or other directly tangible incentives, he/she answered "Affiliates! For discussion and knowledge exchange." He/she then added that also the interaction with the CC HQ, although non-egalitarian in terms of decision-making power, was always very interesting and fruitful as an intellectual or professional/scholarly exchange of opinions.

Other individuals interviewed, although less critical (and explicit), agreed that the governance of CC is "inclusive," but also highly "centralized" in terms of final decision making. The interviewees unanimously stated that the fact that decisions are carefully and publicly documented in writing was a key point supporting participation and open discussion.

\section{Technological Enablers}

Creative Commons is enabled through a variety of means. Here, the definition of enabler is intentionally broad and encompasses everything that supports the good functioning of the $\mathrm{CC}$ network and community. ${ }^{16}$

\footnotetext{
${ }^{15} \mathrm{https}$ ://wiki.creativecommons.org/License_Versions.

${ }^{16}$ The fact that also the interviewed people understood the term "enablers" in a broad way is confirmed by the fact that interviewed CCC staff included in the list of enablers the Board of Directors "who bring expertise, passion, and credibility to our mission" and funders, adding that "more recently, we have been receiving more funds specifically for growing our affiliate network and supporting their goals."
} 
Some simple, but powerful technological enablers are at the core of the $\mathrm{CC}$ network and community. Although not high-tech by modern standards, these technical tools are fundamental for the functioning of the $\mathrm{CC}$ network. In particular, $\mathrm{CC}$ is enabled through its mailing lists. There are lists for license development, community, and technology development, where CCC staff, affiliates, and other stakeholders vet a variety of legal, technological, and adoption-related issues, policy decisions, and other matters. ${ }^{17}$ The translation processes - perceived by CCC staff as "more critical than ever"-are supported by a third-party specialized platform (Transifex ${ }^{18}$ ). In addition, technology and design projects have been increasingly maintained through software versioning tools like GitHub - an example is the recent "State of the Commons" report."

\section{Interaction Between Technical and Non-Technical Stakeholders}

Creative Commons aims at incorporating inputs from both technical (i.e., legally and technologically technical) and non-technical stakeholders in its licensing work.

Within the Creative Commons community, the main debates concern "standardization" of technical legal issues in the licensing domain. However, there are also other relevant technical issues (e.g., technological standardization issues) and less technical issues (e.g., community management and general policy positions).

Technological issues mainly concern the standardization of licensing metadata and understanding relevant technologies - e.g., data mining and big data analytics - as characteristics of the environment in which licenses will be used (mainly, although not exclusively, online). The primary avenue for input on technological matters is the cc-development list. ${ }^{20}$ There, metadata is often a topic of discussion, particularly in recent years. (Recently, Lawrence Lessig, in welcoming Ryan Merkley as the new CCC CEO, highlighted that CC technological standardization projects are not keeping up with the legal tools: "We celebrate the tremendous achievement of Version 4.0 of our Creative Commons licenses. But we are still at Version 2.0 of the technology that we use to deliver those licenses." He added that, "Ryan will fix that,,"21)

To offer a concrete example, during the 4.0 drafting process, $\mathrm{CC}$ solicited input from various stakeholders on digital rights management (called Effective Technological Measures, ETMs, in the 4.0 licenses) and which restrictions imposed by platforms fell within the definition of ETMs. ${ }^{22} \mathrm{CC}$ consulted with the Electronic Frontier Foundation (EFF), among others, on defining the language to ensure it properly captured the types of restrictions that amounted to legal restrictions, which have always been prohibited by $\mathrm{CC}$ licenses. CC also consulted with nontechnical stakeholders on the question of what is appropriate attribution for various disciplines,

\footnotetext{
${ }^{17} \mathrm{~A}$ list of CC mailing lists is available at http://creativecommons.org/contact\#discuss. These lists collect hundreds of messages per year, with peaks during specific discussions. For instance, the number of messages somehow related with the 4.0 drafting process is arguably between 500 and 1,000 .

${ }^{18} \mathrm{https}: / /$ www.transifex.com/projects/p/CC/.

${ }^{19}$ Project online at https://stateof.creativecommons.org/report/; GitHub project at https://github.com/creativecommons/stateofthe.

${ }^{20} \mathrm{http}$ ://lists.ibiblio.org/mailman/listinfo/cc-devel.

${ }^{21} \mathrm{http}: / /$ creativecommons.org/weblog/entry/42705.

${ }^{22} \mathrm{https}$ //wiki.creativecommons.org/4.0/DRM.
} 
including for scientific data (which the 4.0 licenses now more clearly address) and in the educational and scholarly context (e.g., with respect to open educational resources).

In conclusion, not only does $\mathrm{CC}$ solicit contributions from both technical and non-technical stakeholders on their domains of expertise, but it even seeks input on technical issues from nontechnical stakeholders, where their contributions can be helpful.

\section{Interaction Between Geographic Spheres}

Some issues are passed between the different geographic spheres of the CC network-e.g., an issue is identified by an affiliate, brought to the attention of CC HQ, comes back to a group of affiliates, and is then discussed in the general mailing lists.

CC does not have a specific policy to manage these processes, other than seeking input on particular issues from those most affected by them and from those with the greatest expertise.

Many legal copyright issues are global in nature, particularly considering that the online environment is the main (although not exclusive) target of CC licenses. Moreover, Creative Commons is striving to increase the standardization of its legal tools, making them directly applicable worldwide, without the need for local adaptations (such adaptations - the porting process, using CC jargon-were necessary for licenses using versions 3.0 or older). For the time being, however, localized conversations are still necessary for many topics. The best example of this will be discussed below, detailing the problems concerning the licensing of sui generis database rights and how those were discussed during the 4.0 licensing process. The licensing experts on the topic were largely from the European Union, where those rights predominantly exist. During the versioning process, $\mathrm{CC}$ sent several questionnaires to experts in the EU to discern the nature of the sui generis right, which exclusive rights it covers (and what it does not cover), and its exceptions and limitations. CCC staff members then brought the output of this preliminary (but very detailed) conversation to the larger affiliate community.

\section{i. $\quad$ Scalability}

Within the CC network, issues are transitioned both down-sphere (from larger to smaller geographic spheres) and up-sphere (from smaller to larger geographic spheres). Hybrid examples are actually commonplace: specific issues may be identified at the local level, transition upsphere, and again down-sphere, e.g., if there are concerns that a specific policy challenge may spread to other jurisdictions.

Awareness of issues often starts at the local level with an affiliate or community member surfacing a matter of particular importance to them based on a development in their locality or region. These issues are sometimes raised to the full network and to $\mathrm{CC} H \mathrm{HQ}$, and sometimes just locally through the regional email lists. The Regional Coordinators and other CCC staff watch for those issues and bring them to the attention of other regions, the entire Affiliate Network, or other HQ staff as necessary.

One example of this process is the proposed Trans-Pacific Partnership (a proposed trade agreement with potential impacts on copyright protection in multiple countries), which several affiliates flagged as a concern. This concern was elevated to other affiliates in jurisdictions involved in the negotiations, and ultimately CC HQ took policy stances on both the process and substance of those negotiations. 
At other times, issues are identified and raised by CCC staff or the Regional Coordinators, and then brought to the attention of the affiliates and broader community. License compatibility is an example of such a situation. Following the publication of the version 4.0 licenses, CCC established ShareAlike compatibility processes and criteria in an effort to address interoperability problems among silos of incompatibly licensed content. The first candidate, Free Art License (FAL) version 1.3, surfaced several matters of policy that would affect the entire Creative Commons community. Those issues have been discussed and pushed down-sphere for consideration by Affiliates. A second example is work on the World Intellectual Property Organization's proposed Treaty on the Protection of Broadcasting Organizations, which would give broadcasters extensive controls over content they broadcast, even in cases where copyright belongs to others. ${ }^{23}$ This treaty would have a global impact and could adversely affect the ecosystem in which CC licenses operate. In this instance, CC staff surfaced and vetted the issue with the affiliates before advancing to a final decision to sign onto statements opposing the progression of the treaty within WIPO.

\section{ii. $\quad$ Policy Matters and Geographic Spheres}

On matters of policy, $\mathrm{CC}$ tends to work regionally rather than globally when a policy is specific to a region. As CCC staff members put it:

"We do, however, strive to inform and solicit any relevant input from the remainder of the network because local or regional policies can have ripple effects in other regions and worldwide. There have been increasing calls for $\mathrm{CC}$ to take policy stances on certain topics, for example, but generally $\mathrm{CC}$ has been apprehensive to jump in headfirst on this. Our engagement on these matters are informed through interaction by local affiliates on the ground - whether and how CC should respond, is there a more effective organization for doing so [...], and similar. One recent example is the ["Public Consultation on the review of the EU copyright rules"]. The issue was raised by several CC affiliates in Europe, and Creative Commons took notice. The coordination and organization for the input came from the European Regional Coordinators, who are paid CC staff and who in turn solicited responses and input from the affiliates."

In fact, because of a growing number of calls for $\mathrm{CC}$ to take policy stances on various topics (generally related to copyright reform), during the spring of 2013 CCC established (in consultation with the affiliates) some explicit Policy Advocacy Guidelines. ${ }^{24}$ Such guidelines promote policy interventions at a local or regional level, and attempt to clearly differentiate between statements from the CCC as opposed to affiliate teams. The hope was that this would empower affiliate teams to speak on their own. Such an approach aims at making it possible for some affiliates to be very active in the policy space (for example, in the Netherlands, Poland, and Colombia, extremely active and well-connected individuals already interact on these issues as a part of their other work or projects). At the same time, CCC can directly take policy stances only in a few defined cases, with the active involvement and final explicit approval of the CCC Board.

In addition to its affiliates, Creative Commons works with other organizations on both formal and informal terms. $\mathrm{CC}$ has been a member of the Communia Network (now Communia

\footnotetext{
${ }^{23}$ Electronic Frontier Foundations, "Broadcasting Treaty," https://www.eff.org/issues/wipobroadcasting-treaty.

${ }^{24}$ See https://wiki.creativecommons.org/Policy_Advocacy_Guidelines.
} 
International Association) on the Public Domain since its beginnings, LAPSI (The European Thematic Network on Legal Aspects of Public Sector Information, and its continuation, LAPSI 2.0), and WIPO (as an observer on the Committee on Development and Intellectual Property). Many CC affiliates are members of these same organizations. To the extent these organizations have a focus or expertise and formulate policy and other interventions, $\mathrm{CC}$ works through them rather than on its own.

\section{Decision-Making Process}

As described above, ultimate decision-making power always rests with Creative Commons Corporation. The strategic discussions related to legal tools and policies for Creative Commons are driven by the General Counsel, with the approval of the CEO, and the input of staff and the Affiliates. According to the Affiliate Agreements, CCC is required to provide a comment period for any change in policies that affect the Affiliates' relationship with Creative Commons. Those policies include the Privacy Policy, Terms of Use, Trademark Policy, Merchandising Policy, the Legal Code and Translation Policy, and similar policies. Those policies are reviewed annually and updated as appropriate. Similarly, the Affiliate Agreements-which formalize CC's relationship with affiliate institutions and individuals in more than 70 jurisdictions-are regularly updated and vetted with the Affiliates prior to finalization. ${ }^{25}$

With respect to the policy activity of $\mathrm{CC}, \mathrm{CCC}$ applies the aforementioned Policy Advocacy Guidelines. These detail how Creative Commons makes decisions related to advocacy activities, the means by which affiliates may engage in advocacy activities, and various consultation processes related to both. For instance: "The CC Affiliates are notified [...] about the policy action 24 hours in advance (if feasible) of public communication of that decision. Local affiliates should be consulted when potential policy and advocacy actions originate in their respective affiliate jurisdiction. Local affiliates should assist (and potentially lead) on issues where they have demonstrated expertise."

While these formal policies exist, and will ultimately rule in case of controversies, a common perception of the CCC staff and of the members of affiliate institutions is that, within the CC network, most decisions emerge from open discussions within the Affiliate Network or with relevant stakeholders at large. Most of these discussions take place on the CC mailing lists (possibly with parallel regular email discussions within sub-groups, which are then "merged" into the main mailing-list discussion). As CCC staff members put it: "Creative Commons strives for general consensus, though that cannot always be achieved," and "rough consensus" is the preferred plan, whenever possible. In practice, the usual approach is to engage in informal consensus building (including through bilateral discussions between $\mathrm{CC}$ HQ and selected affiliates), with final public discussions on mailing lists, where everybody may assess the level of consensus reached. Some variations are also possible, e.g., when a final version is nearly ready, participants are sometimes asked to voice only strong dissent and/or raise only new severe problems, rather than to postpone reaching consensus.

$\mathrm{CC}$ is currently undertaking a "more concerted effort to act as a true global network, and not make decisions unilaterally," One example is the aforementioned policy/advocacy guidelines. When rough consensus cannot be achieved, CCC acts as steward of the CC tools and overall

${ }^{25}$ See Agreements and Policies on the CC wiki: https://wiki.creativecommons.org/Affiliates. 
mission and makes decisions based on that mission and the community's shared vision. In these cases, CC HQ relies on a subset of "go-to" expert affiliates based on trust built over the years, as well as those with specific subject area expertise.

\section{The Role of Documentation and Institutional Memory}

An element that emerged in both the interviews with CCC staff and affiliate members is the importance of written documentation and institutional memory. In particular, extensive written documentation of the costs and benefits of the options considered and the reasons for the final decision seems to have minimized any negative responses when decisions are made in a more top-down manner.

The role of institutional memory is described in the following way by the interviewed CCC staff members:

"There is a lot of good to having a network with a long institutional memory - and CC has that, for the most part, since we still have several of our original affiliates involved. The same can be said about the makeup of our Board of Directors where some of the most important policy and other decisions are vetted or reviewed-their ongoing presence provides institutional memory and long-term consistency of vision for the organization and the global copyright ecosystem and its challenges. CC staff, on the other hand, does continue to evolve and with that attrition comes some loss of long-term memory. CC does ensure long-term memory and consistency of position through mechanisms other than its affiliates, board and staff, however. We have a long-standing policy of documenting all important policy decisions and product decisions (licenses, public domain tools, deprecation of legal tools, etc.) on our weblog, and through explanatory emails sent to publicly archived and accessible email lists and for affiliates through our affiliate email list."

Institutional memory also helps in facilitating reconsideration of past decisions. Using the words of CCC staff members: "Some decisions can be analyzed and decided based on prior experience with similar or same challenges. One good example of this was the change in CC's position on sui generis database rights. While we had for many years taken the firm stance that our licenses should not be used to license those rights, our experience and engagement with our affiliates in Europe taught to reconsider after many years of experience with that position."

\section{Conflict Management and Time}

There aren't many examples of situations in which the CC network faced a crisis with no viable apparent solutions. The most relevant instance is arguably that of CC's position on sui generis database rights (i.e, a special intellectual property right on databases specific to the European Union). This issue is an example of a major policy change and it will be detailed below, in describing the drafting process that led to version 4.0 of the CC PLs.

Here, it is also worth mentioning that, sometimes, deferring a decision may be an option, as long as the various merits of other options are documented and the existing discussion is not lost. As the interviewed CCC staff members put it,

"We should note that there are at rare times challenging issues that may sit for awhile or require a more in depth consultation before movement can be made. One excellent 
example of this was the creation of $\mathrm{CC} 0$. When first introduced to the affiliate network at CC's 5th birthday party in December 2007, it faced extremely strong opposition. Following the iSummit in Sapporo, CC agreed to table its development and publication while investigating more thoroughly its demand and its effectiveness in various jurisdictions. CC surveyed its legal affiliates, engaged additional legal experts, and painstaking rewrote the legal tool to address many concerns, all over the course of the next $1 \frac{1}{2}$ years. Only upon that further vetting and effort was the community able to generally agree (rough consensus) to lend its support to the new legal tool. To this day, there are a few affiliates who are opposed to its use in some jurisdictions; however, there is general respect for such legal tool and its viability and utility in most all other jurisdictions."

\section{The CC 4.0 Versioning Process}

Since the launch of the first CC PLs at the end of 2002, CC has labeled its licenses with version numbers, as is customary for software project releases. Version 3.0 came out in early 2007, while version 4.0 was released in November 2013.

The 4.0 drafting process ("versioning," using the CC jargon) is described here, as it provides concrete examples of the operation of CC's governance structure.

\section{A. Background and Brief History of the 4.0 CC PLs}

The discussion of Version 4.0 of CC's core license suite began at CC's Global Summit 2011 in September 2011 The discussion was recapitulated and expanded to the CC Affiliate Network mailing list in the following months. The public discussion with the broader CC community and with third parties began in December 2011 with a blog post laying out some of the key reasons for developing a new version of the CC PLs. ${ }^{26}$ Various issues were discussed in the blog post, but a key point was the following: "version 3.0 is working (and will continue to work) really well for many adopters, but [...] [t] he treatment of sui generis database rights in the 3.0 licenses continues to be a show-stopper for many, including governments in Europe. This fosters an environment in which custom licenses proliferate, inevitably resulting in silos of incompatiblylicensed content that cannot be maximally shared and remixed."

The sui generis (or database) right grants an exclusive right on databases when a substantial investment is required to obtain, verify or present the database contents. Such right has been established in Europe according to the Directive 96/9/EC of the European Parliament and of the Council of 11 March 1996 on the legal protection of databases (the "Database Directive"). According to art. 7.1 of the Database Directive, the maker of a database is granted the right to prevent "extraction and/or re-utilization of the whole or of a substantial part, evaluated qualitatively and/or quantitatively, of the contents of that database," The SGDR's term of protection is fifteen years.

The use of licenses to enable releasing databases as open data demonstrates that unmet demand quickly generates new licenses. In particular, until recently Creative Commons-because of the position of the Science Commons group - did not want to explicitly adapt its suite of licenses to

${ }^{26}$ See http://creativecommons.org/weblog/entry/30676. 
databases, with the exception of the public domain waiver/dedication CC0. Science Commons argued - not without reason - that data should be and remain in the public domain, taking a de facto policy stance against the European sui generis database right. ${ }^{27} \mathrm{CC}$ PLs pre-dating the 3.0 version did not mention the sui generis database right; ${ }^{28}$ therefore, it was unclear if the CC licenses (apart from $\mathrm{CC} 0$ ) were an appropriate legal tool for the licensing of databases potentially protected by the sui generis right.

When the localized (ported) European 3.0 licenses were prepared, Creative Commons required a clear stance against licensing the sui generis right. ${ }^{29}$ Ports of the 3.0 licenses for European jurisdictions explicitly addressed these rights as within the scope of the license, but then affirmatively waived all conditions of the licenses for uses implicating the sui generis right. Said another way, the licenses covered copyright and sui generis database rights, but the conditions only applied to uses implicating copyright that could exist for databases based on selection and arrangement, but not other uses. In practice, the European ported licenses attempted to neutralize the sui generis database right and treat them the same as the rest of the world (where the sui generis right does not exist). Therefore, until the 4.0 licenses were released in 2013, the demand for a full-fledged open license for databases in Europe was still unmet.

It appears that because of CC's hesitancy to adapt its licenses to databases, in 2010, the UK government decided not to adopt $\mathrm{CC}$ licenses for the release of its public sector information. UK officials clearly stated in informal discussions that the government was ready to adopt very liberal licensing approaches, but that waiving rights was not an option (i.e., such an approach was perceived as unnecessarily radical in comparison to liberally licensing the same rights). ${ }^{30}$

Moreover, some open data projects, such as Open Street Map (OSM), started to question whether licensing their data with $\mathrm{CC}$ licenses was the best approach. In this case, OSM wanted to adopt a copyleft approach, using existing rights to enforce a viral chain of sharing, and waiving rights to court did not seem to fulfill that objective.

OSM decided to use the Creative Commons Attribution ShareAlike license for its project. However, because it waived and did not license the sui generis right, some in that community believed OSM's steps were inadequate. In response to the partially unmet demand for standard licenses for the legal management of databases in the European legal environment, the Open

${ }^{27}$ Even the "First evaluation of Directive 96/9/EC on the legal protection of databases" (available at http://ec.europa.eu/internal_market/copyright/docs/databases/evaluation_report_en.pdf) published by the European Commission in 2005 is quite critical of the sui generis right and finds that the economic impact of such right is, at best, unproven.

${ }^{28}$ For completeness, there were some 2.5 ports that did mention the sui generis right (and/or implicitly included such right amongst neighboring rights), but these may be regarded as national exceptions not governed by a systematic policy.

${ }^{29} \mathrm{See}$ the treatment of sui generis database rights policy: https://wiki.creativecommons.org/images/f/f6/V3_Database_Rights.pdf.

${ }^{30}$ For the sake of completeness, this was the most widely publicly discussed reason to develop the UK Open Government License. However, other reasons probably included the desire to use a national branded license (a phenomenon that some open data activist named "license vanity"), and the opportunity to address some standard worries of public officials (e.g., misrepresentation, data protection) with custom language. 
Data Commons project was launched. In 2006, the software company Talis published the first public license specifically targeting open data, the Talis Community License, and then funded the drafting of the Public Domain Dedication and License (PDDL). This activity triggered the creation of the Open Data Commons project, which is currently part of the project portfolio of Open Knowledge. In the same time period, the UK Government released its Open Government License, which was followed quickly by the French License Ouverte and the Italian Open Data License. $^{31}$

It is in this complex scenario that Creative Commons began its 4.0 versioning process, with the clear goal of positioning itself as the "one stop shop" for open licensing including open data licensing, and with the only exception of free and open source software licensing.

\section{B. The Governance of the 4.0 Versioning Process}

The international license and legal tool development process is documented by Creative Commons on its official website: ${ }^{32}$

- on the license version page;

- in the Statement of Intent for Attribution-ShareAlike Licenses, which also details the core stewardship activities of CCC; and

- for the 4.0 versioning process, on the 4.0 processes page.

Among other responsibility, the CC General Counsel leads the license versioning and development processes, as well as the development and versioning of other legal tools. As with other relevant processes, the CCC Board also supports the process by providing guidance on overall strategy and strategic decisions on core features of the legal tools.

CCC gathers input from affiliates, the community, and other stakeholders through a variety of means. A public, editable wiki allows anyone to submit issues for consideration, to comment on open matters, and for CCC staff to document decisions and underlying considerations.

The primary discussion forum for proposed changes to the legal tools is the license development list. $^{33}$ This list is a moderated, subscriber-based list that is publicly archived. CCC also solicits input directly from its Affiliates on their dedicated email list (accessible to affiliates only). During the 4.0 versioning process, CCC also obtained input via periodic, regional conference calls held in conjunction with the release of each draft. Outcomes from those calls were publicly posted with the corresponding draft. ${ }^{34}$

\footnotetext{
${ }^{31}$ For more details, see Morando, F. (2013). Legal interoperability: making Open Government Data compatible with businesses and communities. JLIS. It, 4(1), 441.

${ }^{32}$ As one may notice from the links below, it is the "wiki" third-level domain that hosts this part of the $\mathrm{CC}$ website, therefore affiliates and other members of the $\mathrm{CC}$ community may contribute to the description of these processes - with the exception of the " $\mathrm{CC}$ Attribution-ShareAlike Intent" page, which is protected from direct edits. In practice, however, these sections are mainly curated by the CCC staff, and are posted for draft in advance of finalization, as documented by the history of each page on the wiki.

${ }^{33} \mathrm{http}$ ://lists.ibiblio.org/mailman/listinfo/cc-licenses.

${ }^{34}$ For examples, see https://wiki.creativecommons.org/4.0/Drafts.
} 
During the drafting process, $\mathrm{CCC}$ also aimed to identify additional stakeholders beyond those represented or contacted by the $\mathrm{CC}$ affiliates, including candidates for adopting CC's tools or adopters who might be affected by the changes. As described by CCC staff: "These stakeholders are identified by staff and by affiliates and their input may be sought through private engagements rather than public consultations (though $\mathrm{CC}$ takes pains to document all input and decisions that are made). We believe that different stakeholders have different comfort levels with public engagements for any number of reason-getting their input by whatever means is important as long as we remain transparent about interests at stake and reasons for decisions that are based on private rather than public input."

As CCC staff members put it, "The increase in participation and our efforts is ostensibly the reason the [4.0 versioning] process took three full years." Such processes were also conducted at a worldwide scale, thanks to the support of Affiliates and Regional Coordinators.

\section{Final Decisions on the Most Heated 4.0 Drafting Issues}

The key development in version 4.0 was the reversal of policy regarding sui generis rights. Although CC was motivated by the best of intentions, the decision to waive sui generis rights created more problems than it solved. After many years of experiencing problems associated with this decision, and with the input of affiliates and other experts in the EU, ${ }^{35} \mathrm{CCC}$ changed its position, as reflected in the final version 4.0 licenses. In fact, the sui generis right is currently licensed in analogy to copyright, instead of waived. In the end, as CCC staff members put it, "that matter resolved itself, and it is testament to $\mathrm{CC}$ that it can change policy direction when it becomes apparent that an existing policy stance is no longer the best policy for the organization."

Another issue that surfaced most during the 4.0 versioning process concerns the decision whether to define further the meaning of "NonCommercial." Again, the CC community was divided on this issue. In this case, the CCC staff made the ultimate decision to leave the definition as is in order to maintain the expectations of licensors and licensees. As CCC staff members put it, this is a good example of an agreement to disagree. "Our experience has been that so long as we provide a forum in which all sides can be heard, we engage in a healthy debate and demonstrate an understanding of concerns on all sides, that the community respects decisions that are made by $\mathrm{CC}[\mathrm{C}]$ in its stewardship capacity."

\footnotetext{
${ }^{35}$ As described by CCC staff members, "Creative Commons with some frequency consults with third parties outside the network on both policy and legal matters. This includes consulting with affiliates in their other capacities. CC occasionally engages outside legal counsel to obtain formal opinions on legal issues. This is not because our legal affiliates are not adequate, but because they are volunteers, are time-constrained at times, and are not providing formal legal advice to CC (there is no attorney-client relationship). CC also works closely with other organizations that are experts on issues where we may not have expertise within our network or because of the experts' prominence on particular issues. For example, during the 4.0 versioning process CC consulted with EFF on the WIPO Copyright Treaty and the crafting of the definition of Effective Technological Measures, because of their unique expertise in the area from both a policy and legal perspective."
} 


\section{Conclusions and Lessons Learned}

The main lesson of the Creative Commons governance model is best summarized by a quotation from the interviewed Creative Commons staff members, Diane Peters and Timothy Vollmer: "Being overly consultative and overly inclusive, even when there is little response, is important to maintaining credibility as an organization. Similarly, transparency is important, as is being willing to admit we don't have all the answers or always get every decision right. These are important features of a multistakeholder, global organization operating on the cutting edge of legal and policy matters." 


\section{Appendix D: \\ Fighting Spam the Multistakeholder Way - A Case Study on the Port 25/TCP Management in the Brazilian Internet}




\title{
NoC Internet Governance Case Studies Series: Fighting Spam the Multistakeholder Way - A Case Study on the Port 25/TCP Management in the Brazilian Internet
}

\author{
Ronaldo Lemos, Carlos Affonso Souza, Fabro Steibel, and Juliana Nolasco \\ Institute for Technology and Society at Rio de Janeiro State University
}

Editorial Note: Context, Character, and Purpose of the Case Study

This case study is part of a globally coordinated, independent academic research pilot project by the Global Network of Interdisciplinary Internet \& Society Research Centers (NoC). Facilitated by the Berkman Center for Internet \& Society at Harvard University, this study examines existing multistakeholder governance groups with the goal of informing the future evolution of the Internet governance ecosystem. Building upon the NETmundial Principles and Roadmap, it contributes to current policy debates at the international level, including the Internet Governance Forum, the NETmundial Initiative, and other organizations and efforts.

Internet governance is an increasingly complex concept that operates at multiple levels and in different dimensions, making it necessary to have a better understanding of both how multistakeholder governance groups operate and how they best achieve their goals. With this need in mind, at a point where the future of Internet governance is being re-envisioned, colleagues from several $\mathrm{NoC}$ institutions around the world have written twelve case studies examining a geographically and topically diverse set of local, national, and international governance models, components, and mechanisms from within and outside of the sphere of Internet governance. Key findings from these cases are summarized in a synthesis paper, which aims to deepen our understanding of the formation, operation, and critical success factors of governance groups and even challenge conventional thinking.

The research, based on twelve case studies, suggests that there is no single best-fit model for multistakeholder governance groups that can be applied in all situations. Rather, it reveals a range of approaches, mechanisms, and tools available for both the formation and operation of such groups. The analysis demonstrates that the success of governance groups depends to a large degree on the careful selection, deployment, and management of suitable instruments from this "toolbox." As governance groups pass through different phases of operation, conveners and facilitators must remain alert to changes in circumstances that necessitate adjustments to the approaches, mechanisms, and tools that they deploy in order to address evolving challenges from inside and outside. This case study provides insights into how those instruments can be deployed and adjusted over time within such groups, and highlights how their interactions with important contextual factors may be successfully managed within given resource restraints.

The research effort is grounded in a diversity of global perspectives and collaborative research techniques. Adhering to objective and independent academic standards, it aspires to be useful, actionable, and timely for policymakers and stakeholders. More broadly, the Network of Centers seeks to contribute to a more generalized vision and longer-term strategy for academia regarding its roles in research, facilitation and convening, and education in and communication about the Internet age.

For additional information on the initiative, please contact Urs Gasser, Berkman Center for Internet \& Society, at ugasser@cyber.law.harvard.edu. 


\begin{abstract}
This case study explores how CGI.br, the multistakeholder Brazilian Internet Steering Committee, addressed through a collaborative decision making and educational process the difficult issue of spam propagation. Although the technical solution to the spam problem in Brazil was relatively clear, convincing stakeholders to adopt the solution was challenging. Telecommunications companies and ISPs initially resisted this recommendation out of concern around the costs of switching and the challenges of communicating the change to end users. This case study examines how a multistakeholder process involving telecommunications companies, ISPs, consumer rights associations, and government ministries and agencies was able to overcome these obstacles while simultaneously respecting consumer rights, freedom of speech, and commercial competition. CGI.br's Anti-Spam Working Commission (CT-Spam Commission) highlights how collaborative governance can be applied in an iterative and educational fashion. Addressing the spam issue would require buy-in and cooperation from a variety of parties, and by engaging all stakeholders, the CTSpam Commission was able to identify the concerns of stakeholders and then develop a variety of educational materials, technical reports, and policy changes in order to address those concerns. By developing the policy in such a fashion, the CT-Spam Commission was able to gain the support of the telecommunications companies and ISPs without regulatory oversight. Ultimately, with the buy-in of key stakeholders, implementation of the Port 25/TCP recommendation in 2013 led to a dramatic decrease in spam in Brazil.
\end{abstract}




\section{Table of Contents}

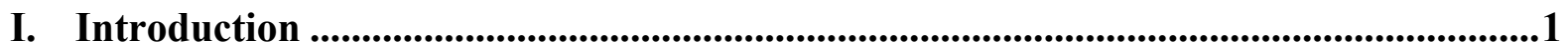

II. Identifying a Policy Issue .....................................................................................................2

A. Notes On the Structure of CGI.br ..................................................................................................... 2

B. Spam as a Technical Policy Issue: 2005-2009............................................................................ 4

C. Spam as a Broad Policy Issue: 2009-2011 ...............................................................................5

III. Research as a Policymaking Aid ...................................................................................6

A. Measuring the Problem Size .............................................................................................. 7

B. Informing the Community ...............................................................................................9

IV. Consensus-Building Measures ...........................................................................................9

V. Results and Discussion .....................................................................................................10 


\section{Introduction}

For over fifteen years Brazil has developed a model of multistakeholder Internet governance. There is already substantive literature covering the process of creating the Brazilian Internet Steering Committee (CGI.br), but less attention has been dedicated to describing how a multistakeholder decision-making process has been implemented and fostered by CGI.br, or to exploring its peculiarities and challenges.

The fight against spam, and more specifically the adoption of Port 25/TCP management on the Brazilian Internet, is a case study that is worth exploring due to the elements it pulls together, providing a complex and in-depth picture of the multistakeholder process in practice. In 2005, CGI.br, through its Anti-Spam Working Commission (CT-Spam), ${ }^{1}$ began addressing the issue of spam in Brazil. Since then, CT-Spam has produced a number of academic studies and technical reports that have supported the adoption of Port 25/TCP management as the most effective measure to fight spam. ${ }^{2}$

The initial efforts of CT-Spam focused on the technical aspects of limiting spam. Its recommendation for telecommunication companies (telcos) and Internet service providers (ISPs) alike was to replace Port 25/TCP — a TCP port with low security standards - with Port 587/TCP, which has higher standards. Telecommunications companies and ISPs, however, did not adopt the recommendation outright, and in 2009 Brazil was first on the list of countries that send out the largest amount of spam, according to the Composite Blocking List (CBL). ${ }^{3}$ The international press called Brazil the "new king of spam," and the issue also made headlines in national media. ${ }^{4}$ In order to reverse this situation, in 2009 CGI.br changed its tactics and started to attack the issue more broadly. Instead of treating spam as a purely technical Internet infrastructure problem, they addressed it as a regulatory issue that concerned consumer rights, freedom of speech regulation, and commercial competition. This approach led to the 2013 implementation by telcos and ISPs of the technical recommendation formulated first in 2005, and reformulated in 2009. Since then, Brazil's spam ranking has dropped drastically.

Brazil was one of the first countries to attempt to regulate Port 25/TCP, nonetheless, it took more than seven years for the issue to be solved. In the meantime, countries such as the United States, Japan, and the European Union implemented the same recommendation in much shorter timeframes. ${ }^{5}$ In contrast to CGI.br's multistakeholder model, these countries and regions opted for a more government-oriented decision-making process, which suggests that the multistakeholder approach adopted in Brazil is less efficient than the more top-down initiatives adopted elsewhere. There are, however, two arguments which counterbalance this fact: first, the

\footnotetext{
${ }^{1}$ Comissão de Trabalho Anti-Spam. More information available at http://www.cgi.br/acoes/antispam.htm\#a4 (accessed on 01.Jul.2014). The working group started on 14 January 2005.

${ }^{2}$ HOEPERS, C. JESSEN, K. Gerência da Porta 25: Motivação, Importância da Adoção para o Combate ao Spam e Discussões no Brasil e no Mundo, 2009. http://www.cert.br/docs/ct-spam/ct-spam-gerencia-porta-25.pdf (accessed on 01.Jul.2014).

${ }^{3} \mathrm{http}: / /$ www.nic.br/imprensa/clipping/2013/midia182.htm.

${ }^{4}$ Composite Blocking List, http://cbl.abuseat.org/country.html (accessed on 01.Jul.2014).

${ }^{5}$ Japan Email Anti-Abuse Group: http://jeag.jp/index.html; Rubenking, Neil. Port 25 Block Stalls Spam After All, http://securitywatch.pcmag.com/spam/290791-port-25-block-stalls-spam-after-all; BEVERLY, Robert; BAUER, Steven; BERGER, Arthur. "The Internet's not a Big Truck: Towards Quantifying Net Neutrality," http://www.akamai.com/dl/technical_publications/truck-pam07.pdf.
} 
multistakeholder process had an intrinsic value in connecting different stakeholders in a joint effort; second, particular characteristics of the Internet regulatory environment in Brazil made it so that no stakeholder alone coulc implement the necessary technical solution without cooperation from others. Thus, a more drastic political approach was likely to face major criticism.

As this case study argues, a top-down regulatory initiative in Brazil was likely to fail. Implementing TCP port management can be done in several ways, but it requires a collaboration of three levels of players: telcos (who control the use of Internet infrastructure), broadband providers and ISPs (who provide connectivity and IP addresses, and/or authenticate users and provide services such as e-mail and web), and Internet users.

In contrast to other countries, in Brazil, telcos, broadband providers, and ISPs are not regulated in the same way. While telcos are closely regulated by Anatel, the other stakeholders are less strictly regulated through various governmental and self-regulatory institutions. If a top-down, government-run solution was implemented, there would be no single agency or decision-making process that could enforce compliance. This is why a multistakeholder approach was more likely to succeed in Brazil.

This long process, conducted in a multistakeholder fashion, is the focus of this case study. Grounded in national and international literature on Internet governance and regulation, as well as in the current status of the multistakeholderism debate, this article is a result of a yearlong series of 11 in-depth semi-structured interviews involving 12 interviewees. We address the following research questions: How were different stakeholders identified, and how have they interacted with each other? What was the role of governmental entities in pushing the process forward, and how did it negotiate power relations between private sector and civil society players? How was consensus reached? What has motivated different stakeholders to engage in Port 25/TCP management?

Research on the process and obstacles to implementing the management of Port 25/TCP in Brazil offers a rich case study for academic analysis. From that, we can make observations about how this process took place, how the different parts interacted, and how consensus was obtained.

\section{Identifying a Policy Issue}

To better understand how the CT-Spam, formed inside CGI.br, came to the conclusion of how it should act to fight spam, it is necessary to first highlight a few structural aspects of the Brazilian Internet Steering Committee as a whole, since they will play a larger role in the discussion around the challenges faced by the anti-spam project.

\section{A. Notes On the Structure of CGI.br}

CGI.br was created by Interministerial Ordinance nr. 147/1995 $5^{6}$ which was amended by Presidential Decree nr. 4,829/20037, "with the purpose of coordinating and integrating all Internet service initiatives in Brazil, as well as promoting technical quality, innovation and the dissemination of the services available". It is a multistakeholder institution comprised of members from the government, the private sector, non-governmental organizations (NGOs) and

\footnotetext{
${ }^{6} \mathrm{http} / / /$ cgi.br/portarias/numero/147.

${ }^{7}$ http://cgi.br/pagina/decretos/108.
} 
the academic community. CGI.br has 21 councilors, 10 of them nominated by the Government and eleven selected through elections to represent the private sector, NGOs and academia in the Council. A representative of the Ministry of Science and Technology coordinates the works of the Council, as established by Article 2 of the aforementioned Decree.

The elected councilors have a three-year mandate and, according to Decree nr. 4,829/2003, they render a public interest service, not being entitled for any remuneration for the time they serve in the Council.

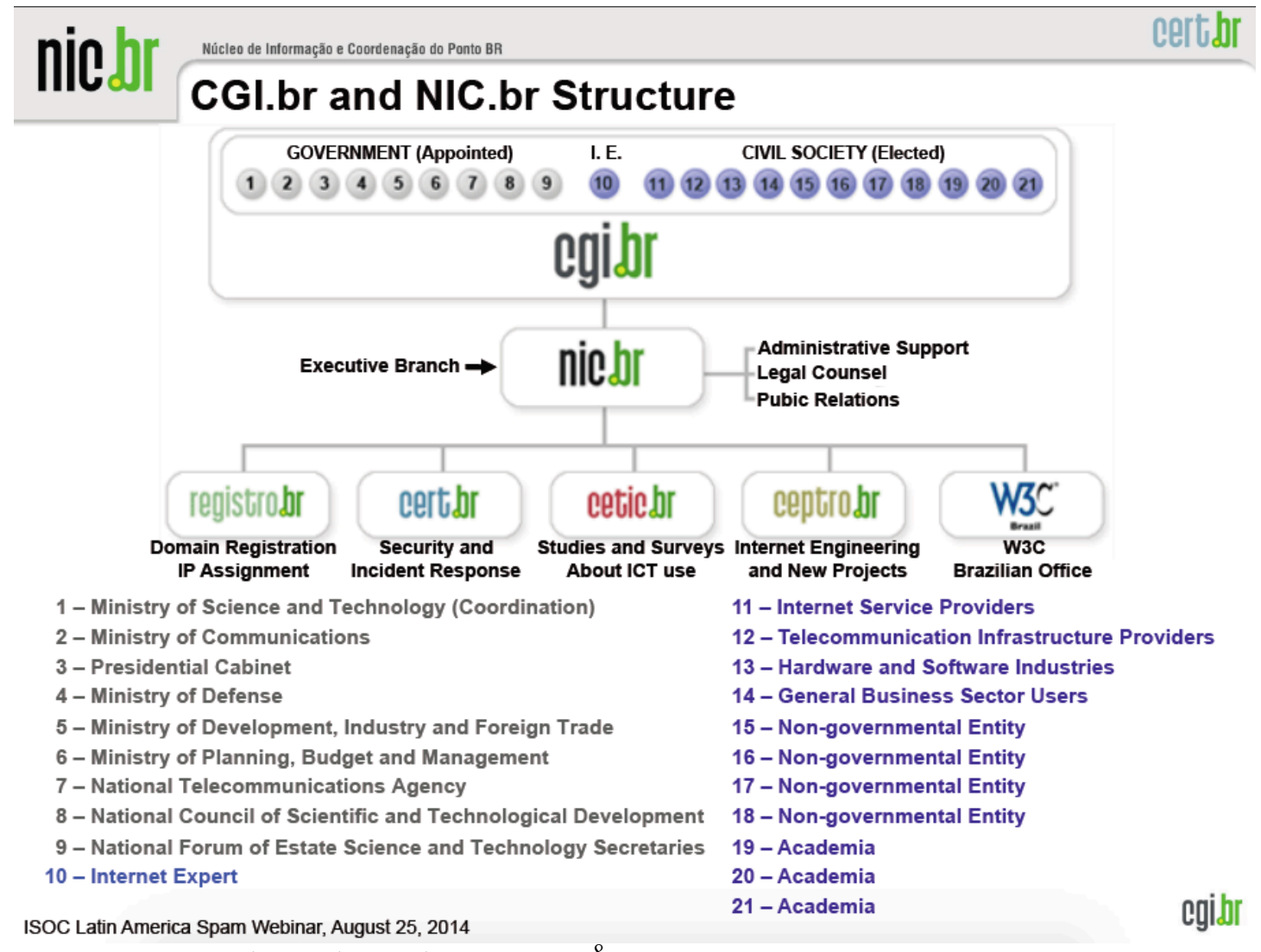

Figure 2 : CGI.br and NIC.br Structure 8

The CGI.br organization increased in complexity along the years, including the establishment in 2005 of NIC.br, the Brazilian Network Information Center, which implements the decisions and projects approved by the Brazilian Internet Steering Committee.

In 2009 CGI.br approved a ten-principle list for the governance and use of the Internet in Brazil. Commonly referred to as CGI.br's Decalogue, ${ }^{9}$ this set of principles offered inspiration for the Brazilian Internet Bill of Rights (approved in the National Congress as Law 12,965/2014) and serves as a guiding reference to a number of initiatives developed by the Council, such as CTSpam and its blocking of Port 25/TCP project. Especially as concerns the anti-spam project as

\footnotetext{
${ }^{8}$ HOEPERS, Cristine. "A multistakeholder effort to reduce spam - The case of Brazil", available at http://www.cert.br/docs/palestras/certbr-isoc-lac2014.pdf.

${ }^{9}$ CGI.br's Decálogo, available at http://cgi.br/en_us/resolucoes/documento/2009/003.
} 
described in this report, the Decalogue's principles of Democratic and collaborative governance, Neutrality of the network and Legal and regulatory environments are instrumental to the better understanding of how the project came into being.

CT-Spam is the anti-spam task force of CGI.br, created in 2005 with the objective to reduce and control spam in Brazil. It was called in reaction to the increased blacklisting of Brazilian broadband providers' IP ranges, a rise in operational costs, the instability of broadband connectivity, and the decrease of consumer quality service associated with the spam problem. Its objectives include "raising awareness about the importance of best practices, data protection and privacy issues related to e-mail marketing, studying a legal framework for Brazil, suggest procedures to combat spam, legal review and promote international articulation to fight spam". ${ }^{10}$

CT-Spam began addressing spam as a policy issue in 2005, offering a technical solution for a technical problem of Internet infrastructure. By then, only CGI.br advisors and academics were involved in the process. Other stakeholders were quickly called in to raise awareness of the topic, but this was not sufficient to implement the plan. By 2009, four years later, telcos, broadband providers, and ISPs alike were reluctant to adopt CT-Spam's recommendations. This reluctance prompted CT-Spam to liaise with the CGI.br Committee Board and to reframe the policy issue more broadly. In 2009, CT-Spam began to relate the technical aspects of Port 25/TCP management to consumer rights, freedom of speech, and economic competition. The result of these actions is the creation of a policy network ${ }^{11}$ that, seven years later, has succeeded in implementing the adoption of Port 25/TCP management to mitigate spam.

\section{B. Spam as a Technical Policy Issue: 2005-2009}

When the issue of spam was brought forward by CT-Spam, two types of spam messages were identified: spam based on its content, and spam based on its source. Content-based spam, which typically originates from individual email accounts without users' knowledge, is a complex and disputed topic, and was perceived as a challenging regulatory issue. CT-Spam analyzed the role of users in spam (users here defined as end users - contract holders with ISP providers) in order to understand the role of spam messages sent by users on purpose (i.e., spammers), and messages sent by users without their knowledge (i.e., victims of spammers' attacks).

To understand that distinction in types of spam, CGI.br conducted an educational campaign with private sector players, ${ }^{12}$ and jointly published an Ethical Code of Conduct. The Code labeled spam messages as UCE (Unsolicited Commercial E-mail) and defined spam as messages that share four characteristics: commercially oriented messages, sent indistinctively to a large groups of receivers, with identical content, and without users' consent. Managing this type of spam involves analyzing the content of all messages. Interviewees noted that if addressed using port

\footnotetext{
${ }^{10} \mathrm{CT}$-Spam website, available at http://antispam.br/en/.

${ }^{11}$ CT-Spam aimed to group Telcos, ISPs, security groups, direct marketing businesses, civil society groups and other segments directly related to the problem, in the technical and operational levels. Amongst those represented in the Task Force were a CGI.br's Board Member (coordinating the group), CGI.br's Concil Board members, NIC.br and CERT.br members, and also representatives from telecom companies, academics and governmental representatives (from the Ministry of the Technology and Science and from Anatel, the Telecommunication Regulatory Agency).

${ }^{12}$ CERT.br, Cartilha spam http://cartilha.cert.br/spam/ . Accessed on 01.Jul.2104.
} 
management, this approach violates certain fundamental principles of human rights (the right to privacy, in particular).

Source-based spam, however, is a much less disputed policy issue. In the case of content-based spam, such as email marketing and chain emails, the user's account is responsible for sending the message to others. As such, the user has (or might have) control of what it says. In the case of source-based spam, however, it is the user's computer (and not their email account) that is responsible for sending the messages. This means that the spam message is not the user's message, but instead is a message sent through the user's computer without the user's intent or knowledge.

As interviewees explained, regular email messages are sent using SMTP (Simple Mail Transfer Protocol), a technical standard that enables users' computers to communicate with email servers in order to link an email access point to an email server. The default outbound port to provide this communication is Port 25/TCP, meaning that all emails a user sends from their computer need to access this outbound port to reach ISPs to be routed to their final destination. It is possible to set up the user's computer to perform both tasks, acting not only as regular email access points but also as an email server.

CT-Spam's policy suggestion focused on the capability of a user's computer to be set up to operate as an email server when Port 25/TCP is used. Each computer, if properly exploited and externally controlled, could send spam bulk messages without the user's notice or control. This also means that ISPs or other Internet hubs cannot effectively fight spam without analyzing a message's content, which invades user privacy and violates human rights standards. Replacing Port 25/TCP with Port 587/TCP would address a default system that offers a low standard of security (i.e., no password requirement) by replacing it with a system offering a higher standard of security. This would drastically reduce the amount of spam going out of the country.

Interviewees describe the technical solution chosen as being crystal clear, but they report having difficulties translating what they saw as a low-cost, highly efficient, easily implemented, and internationally accepted solution into terms easily understood by non-technical communities (such as lawyers, journalists, and public servants). In 2009 CT-Spam was about to drop the case due to the challenges with implementation. They knew beforehand they had to align three levels of Internet infrastructure: end users, who had to manually set up their own computers; ISPs and broadband providers, who had to change their default TCP port and instruct end users on how to set up their own machines; and telcos, who were responsible for blocking Port 25/TCP traffic. But CT-Spam did not anticipate that implementing Port 25/TCP management could not be accomplished through approaching it as a purely technical Internet infrastructure matter.

\section{Spam as a Broad Policy Issue: 2009-2011}

When CT-Spam issued a technical note in 2005 suggesting the replacement of Port 25/TCP with Port 587, it was clear that the policy issue had a strong technical aspect. What was not clear at that point, according to the interviewees, was the broad range of related political, social, and economic aspects that would surface. CT-Spam had several meetings with high ranked technical staff from telcos, broadband providers, and ISPs, who raised several concerns. The first was economic: the private sector wanted to investigate the costs of switching TCP ports, and what hardware implications this would have. There was also a consumer rights concern: if, as a result 
of the switch, users could not access their email accounts, they would blame ISPs and telcos, which could lead to private sector companies being brought to court.

Lastly, there was a broad regulatory concern related to the ecology of Internet regulation in Brazil. While areas such as telecommunications and broadcast are specifically regulated based on constitutional grounds, the Internet is not. The first Internet-related legislative proposal was sent to Congress in 1998. However, only after landmark attempts at regulation were made in 2005, 2008, and particularly in 2009 (when the Brazilian civil rights framework for the Internet, Marco Civil, was drafted) did Internet regulation become a top priority policy issue for government, civil society, and private sector alike.

According to interviewees, telcos were keen to promote Anatel (the Brazilian Agency of Telecommunications) as an important regulatory agency for the Internet, and demanded that Anatel officially support CT-Spam's recommendation. At this point in 2009, however, Anatel's role in CGI.br was unclear. Also, there was a concern that the CT-Spam recommendations would violate net neutrality, a value supported by CGI.br principles (as outlined in the Decalogue) and strongly supported by the Marco Civil. Previous attempts by telcos to block VOIP ports were defined as a violation of net neutrality, and they feared that the same would happen in the initiative to block Port 25/TCP.

Taking the three concerns together (i.e., the economic impact, consumer rights, and the regulatory ecology), interviewees report that only after 2009-when CGI.br's Committee Board joined the negotiation and vice-presidents and legal consultants of private sector companies were invited to join the debate-were the technical aspects of the policy issue able to be considered. After this initial expansion, more governmental bodies and civil society representatives joined the decision-making process, which cleared the way for the technical solution to be implemented in March 2013. As interviewees report, they were all ready for a massive number of complaints from final users who suddenly found they could not access their email. The implementation, however, was done gradually, from one set of cities and ISPs to another, and the overall perception was that the policy was implemented without any major drawbacks.

\section{Research as a Policymaking Aid}

As interviewees clearly point out, Brazil was the first country to officially define source-based spam as a policy problem and issue an official document to address Port 25/TCP management. Between 2005 and the implementation of the policy solution in 2013, however, several other regions took the action Brazil was slow to adopt. Japan, the European Union, and the United States (i.e., Comcast) all switched TCP ports within six months or less, while in Brazil the change took more than seven years. However, interviewees state that the long timeframe to implement the port management was necessary because stakeholders needed plenty of time, information, and knowledge before agreeing to support the policy recommendation.

Brazil has some particular characteristics that made Port 25/TCP management peculiar. As mentioned above, in Brazil, telcos and ISPs are not regulated in the same way, nor are they regulated by the same agency (telcos are regulated by Anatel, and ISPs are softly regulated by Anatel and other institutions). CGI.br is also a unique institution, taking a multistakeholder regulatory approach and focusing more heavily on consultation processes. Within this regulatory framework, CT-Spam had to perform a policy change from a non-binding position, in an 
environment where other agencies had a clear legal mandate to act (though at the same time they could not implement a policy change alone).

To achieve their goals, CT-Spam decided to invest in research initiatives to persuade key stakeholders to approve of the policy change. They approached this on three different fronts. First, they funded research to measure the dimensions of spam in the country, investigating the number of messages involved, the main incoming and outbound destinations, and the amount of Internet traffic consumed. The second component investigated non-technical aspects of Port 25/TCP management, requesting policy reports on the consumer rights and human rights impacts from the Federal Prosecutor's Office, consumer defense organizations, the public servants from the Ministry of Justice, and academics. Lastly, CT-Spam created tools to disseminate the information they generated, aiming to educate and mobilize actors.

All the above aspects were key to the success of the blocking of Port 25/TCP project. Investing in research, for example, provided a key argument for telcos and ISPs to consider, and raised awareness about the topic. After all, if up to $90 \%$ of the Internet traffic they were selling to their clients might be used without their users' awareness, their efforts were being wasted from a marketing perspective. At the same time, major concerns about other regulatory issues kept stakeholders immobile. Only after non-technical issues such as consumer rights and commercial competition were discussed alongside the technical issues did telcos and ISPs feel secure enough to block the Port 25/TCP. Parallel to all these efforts, CT-Spam launched a website to explain what spam was and what needed to be done in order to legitimize the process. It allowed key civil society representatives to advocate for the cause online. This in turn allowed telcos and ISPs to join a well-known cause.

\section{A. Measuring the Problem Size}

In 2005, CT-Spam operated as a working group to measure and identify the key aspects of spam in the country. This initiative was mostly coordinated by CERT.br member Klaus StedingJessen, with the support of CGI.br council member Marcelo Fernandes. Questions asked by the working group included: how much of data traffic is consumed by spam? Are the sources of spam domestic or international? What is the impact of spam on the average user's experience on the Internet? What other services are impacted by the blocking of Port 25/TCP?

To address these questions, in 2006 CT-Spam started to use an in-house research program from CERT.br named HoneyPots. HoneyPots are computers, distributed along the network, that emulate normal Internet use and computer configurations. After some preliminary tests, CERT.br adapted their computers and started to run a specific script to investigate spam (naming the project SpamPots). The SpamPots were run without the awareness of telcos or ISPs to avoid possible external interference, and provided CT-Spam for the first time with quantitative measurements of spam circulation in the country. Over 325 days, the SpamPots collected a total of 370,263,413 messages addressed to more than 3 million users worldwide and originating from 157 countries, most of them from Taiwan and mainland China (the project also found that $90 \%$ of the spam messages were written in Chinese). ${ }^{13}$

\footnotetext{
${ }^{13} \mathrm{http}: / /$ www.nic.br/imprensa/clipping/2007/midia354.htm (acessado em 12.10.2013). Segundo o release de divulgação dos dados da iniciativa Spampots, publicado em 11.07.2007: "A lista dos 10 países que mais abusam do Brasil, de acordo com os resultados preliminares, traz Taiwan em primeiro lugar, com 281.601.310 e-mails
} 
Later on, an academic institution (UFMG) conducted additional research. Over 15 months they used ten computers to provide broad and statistical information about spam in Brazil. The results of this research enabled CT-Spam to make specific policy arguments based on hard data. Summarizing the three main arguments stated by interviewees, it was clear by then that: (a) Brazil was actually a spam hub: a mule, not a spam producer, with local computers being used by outsiders to send messages abroad (mainly to and from China); (b) Because most users in the country used ADSL, which provides an asymmetric connection, the amount of Internet bandwidth used to upload spam messages was seriously compromising their Internet use; and (c) Inbound computer invasions came from several types of IP ports, but all of them used Port 25/TCP for outbound communication. ${ }^{14}$

As the interviewees argue, until these numbers were collected, the technical community was not convinced that spam was an urgent topic. Spam records so far were mostly provided by antivirus and malware software companies, which were perceived by the community as untrustworthy sources. When CT-Spam revealed the numbers to the telcos and ISPs, and reinforced that blocking Port 25/TCP had already been recommended by the IETF, ${ }^{15}$ the technical community agreed on the need to address the issue. There was nonetheless debate over whether Port 25/TCP would be a sufficient solution. As Klaus Jessen stated in an interview:

(...) it was by then clear to us that no matter how many incoming ports were being abused, the outbound port was always the same: Port 25/TCP (...) However, some ISPs counter-argued saying we should also manage inbound ports, but we replied saying: "look, we identified 30 inbound ports being used today for invading users' machines, but there is only one port in use to send spam out, and this is the one used as default port in SMTP configuration" (...) [after that] we thought that exposing that spam was a waste of Internet traffic, and that was bad for ISPs, and that there was a clear solution to address it, they would agree with us. But they kept disagreeing with us, even amongst technical staff.

In order to allow the debate to move forward, CT-Spam engaged CGI.br's Committee Board to research the role of spam particularly with respect to consumer rights. The academic institution Fundação Getulio Vargas developed a policy report on the impact of spam in Internet regulation and Internet fundamental rights, which was written by legal experts including Ronaldo Lemos, Danilo Doneda, Carlos Affonso Pereira de Souza, and Carolina Rossini. ${ }^{16}$ The report concluded that the management of Port 25/TCP was not a threat to net neutrality because it offered alternatives for the same service using an alternative TCP port. They also argued that Port

capturados, ou 76\% das ocorrências. China vem em segundo lugar, com 58.912.303 e-mails ou 16\% do volume analisado. Estados Unidos, Canadá, Coréia, Japão, Hong Kong, Alemanha, Brasil e Panamá são os outros países que aparecem na listagem, e que juntos somam menos de $8 \% . "$

${ }^{14}$ Entrevista de Cristine Hoepers e Klaus Jessen ao projeto de Documentação da Gerência da Porta 25, concedida em 25.09.2013.

${ }^{15}$ Internet Engineering Task Force (a non-commercial and not-for-profit non-governmental organization responsible for developing and promoting Internet Standards)

${ }^{16}$ LEMOS, Ronaldo; DONEDA, Danilo; SOUZA, Carlos Affonso; e ROSSINI, Carolina. Estudo sobre a Regulamentação Juridica do Spam no Brasil. Publicado originalmente em abril de 2007.

http:/www.cgi.br/publicacoes/documentacao/ct-spam-EstudoSpamCGIFGVversaofinal.pdf (acessado em 12.10.2013). Some of these authors are also authors of this case study. 
25/TCP management would improve fundamental rights online, and supported the initiative as a creative solution to address spam without invading privacy.

Moreover, CGI.br also requested a report from the Brazil Secretary of Citizen's Rights (SENACON) on the responsibilities of telcos, broadband providers, and ISPs in Port 25/TCP management. The report concluded that ISPs were responsible for providing information to end users on how to switch TCP ports, but released them from responsibility for problems relating to the port block. They also concluded that users who wanted to have their port set to Port 25/TCP could request it, which enabled telcos to implement the change with minor judicial pitfalls. SENACON was also asked to inform other agencies of the judicial system related to consumer rights of this decision, which interviewees viewed as a safeguard to support the private sector in the event of legal action by users.

\section{B. Informing the Community}

In 2006, CT-Spam launched a website to provide information about spam and Port 25/TCP (antispam.br). Different audiences were targeted through the website: end users could read FAQs and watch educational videos; the technical community could read advanced materials on how to configure computers and private networks; and NGOs could engage in the campaign and share promotional materials. Moreover, as interviewees argue, the portal increased the legitimacy of the campaign, making "official" the public initiative to combat spam. Apart from that, CT-Spam also organized a series of seminars and talks across the country, and used these opportunities to give publicity to the academic and technical research they had been doing. Internationally, CTSpam signed 12 mutual cooperation agreements to conduct research on best practices abroad and to export knowledge on spam internationally. CT-Spam also hosted meetings with telcos, broadband providers, and ISPs to inform them of their research findings. The first meeting participants were technical staff of the private sector, but from 2009 onwards, company vicepresidents and lawyers also joined the meetings.

\section{Consensus-Building Measures}

Although the technical solution for the spam problem was clear and uncontested, the implementation delay of more than seven years is due to a lack of cohesion among stakeholders. In 2005, interviewees reported that the international community was already complaining about the amount of spam coming out of Brazil. Interviewees were aware of the number of Brazilian URLs included in international blacklists. And the topic was appearing more and more frequently in media venues both outside and inside the country. Growing international concern motivated CGI.br to create CT-Spam, to issue the technical note on Port 25/TCP management, and to request best practices from abroad to inform the national debate.

CT-Spam, however, knew that even though the international reputation of Brazil was endangered abroad, the technical solution for spam did not depend on international collaboration. The block of Port 25/TCP was an efficient solution for the spam problem, and it could be implemented by national telcos alone. The work of CGI.br from that point onwards was to liaise with the international community to exchange experiences (for example, signing mutual cooperation agreements), receive international missions (e.g., a delegation from Japan visited Brazil before deciding to manage Port 25/TCP in their own country), and discuss the topics with international networks of which CGI.br was already a member. 
The most challenging effort for CGI.br, however, was build a consensus among key stakeholders inside the country. CT-Spam could not enforce regulations, but only issue recommendations. Since the release of the technical note, however, only a few ISPs had voluntarily managed Port 25/TCP, while major players were mostly reluctant to accept the change. Telcos, for example, insisted that Anatel, their regulatory agency, support the initiative. Anatel's role in CGI.br until 2009 was marginal, and only after the Committee Board officially requested that Anatel support the initiative did telcos begin to change their position. Telcos and ISPs also requested that the Ministry of Justice and the judiciary consumer rights group support the initiative; CGI.br also organized this.

As a result of these requirements, the blocking of Port 25/TCP project support gradually became more and more multistakeholder. In 2010, for example, CGI.br and Anatel signed a cooperation agreement, which resulted in a formal commitment by the telcos to support CT-Spam's recommendations. ${ }^{17}$ In 2011, the Department of Defense and Protection of Consumers of the Ministry of Justice (DPDC/MJ) ${ }^{18}$ issued a technical note to inform judicial institutions about the block of Port 25/TCP, freeing telcos and ISPs of major consumer rights responsibilities (and at the same time assuring that, when necessary, users could request their Port 25 be opened). By then, the project website was established as a source of information and legitimacy, which cleared the way to align all players and implement the full block of Port 25/TCP in the country.

\section{Results and Discussion}

The deadline for the implementation of Port 25/TCP management was March 2013. There was no need to impose penalties or incentives to implement it, mostly because stakeholders themselves paved the way for a smooth transition to take place, and they mutually agreed on a calendar of activities. As a result of the policy implementation, Brazil dropped from first place in the CBL spam ranking in 2009 to $25^{\text {th }}$ place in 2013. The implemented solution has not fully resolved spam issues in Brazil, but it has at least significantly mitigated the problem. As Henrique Falhauber said in an interview:

"The website antispam.br aided us to mobilize and inform the public about the issue of spam. However, spam is still a problem that involves social networks and SMS, for example. Mobilizing and educating people is a fundamental task we continue to perform". ${ }^{19}$

\footnotetext{
17 ofício n 195/2010-PR-ANATEL.

${ }^{18}$ Nota Técnica - NT n ${ }^{\circ} 65$ CGSC/DPDC/SDE, available at http://www.antispam.br/porta25/brasil/notatecnica65.pdf.

${ }^{19}$ Henrique Faulhaber, interviewee.
} 


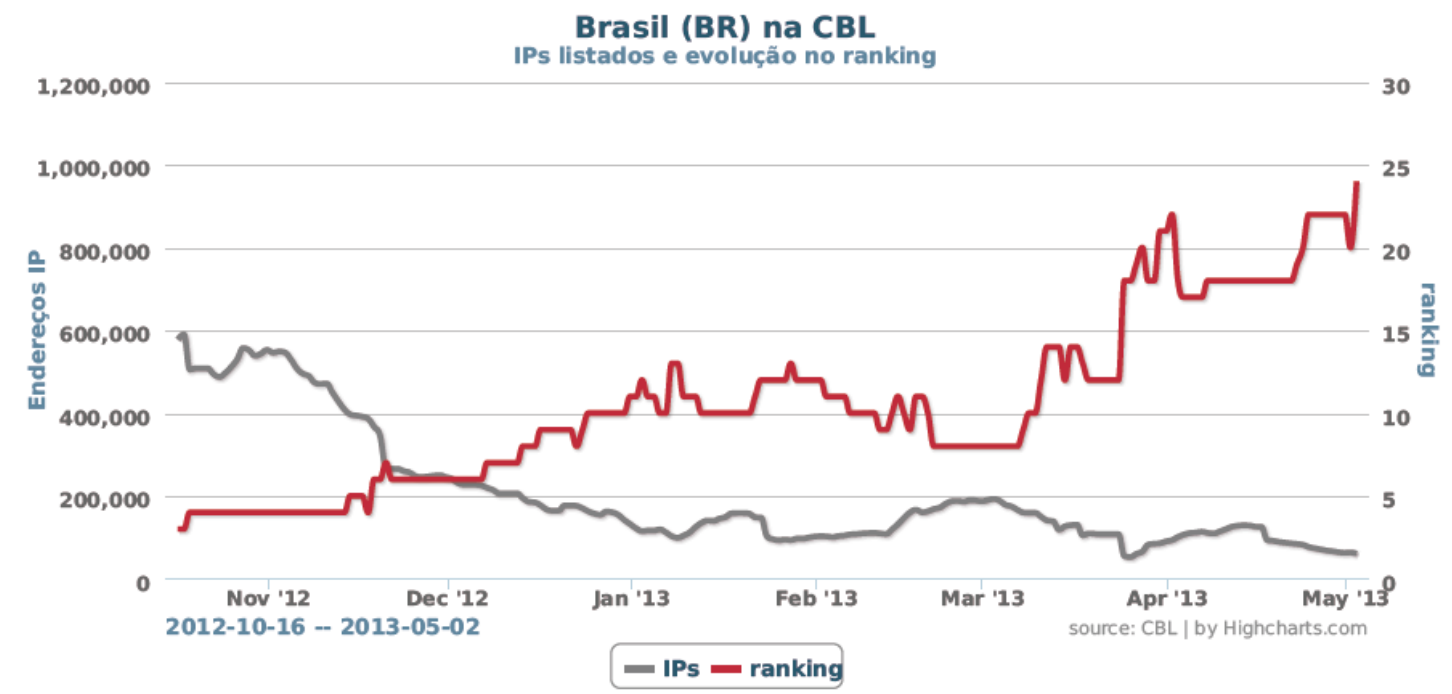

Figure 2. CBL IP blocked addresses vs. Country ranking, Nov 2012 to May 2013 (Source: nic.BR)

We might say however that the blocking of Port 25/TCP project succeeded in two main areas of multistakeholder processes: (a) improving decision-making processes for Internet regulation, and (b) supporting fundamental rights related to Internet use.

The implementation of Port 25/TCP management was highly influenced by the concurrent drafting of the Marco Civil, a bill protecting fundamental rights, including privacy, net neutrality, and freedom of expression, approved as law in Brazil in April 2014 (but drafted collaboratively since 2009). As interviewees argue, Port 25/TCP management was a pre-test of what legal implications Marco Civil would have, and how to solve them. The initiative was therefore a turning point for Internet regulation in Brazil. As Marcelo Bechara argued in an interview, the initiative was the first clear managerial decision-making action of the Committee Board, a case that contrasted with the more deliberative role usually performed by CGI.br.

The blocking of Port 25/TCP project was a managerial-oriented process that should not be mistaken for a top-down government-run initiative. The main policy actor driving the process (CT-Spam) was a multistakeholder group, and the main policy decision-making arena (CGI.br Committee Board) is by definition a multistakeholder body. It is true that not all stakeholders represented in CGI.br participated in the process (for example, no members of the Ministry of Communications, Ministry of Science and Technology, the National Scientific Development Council, or representatives of the Telebras System were mentioned by interviewees as key players). At the same time, other formal members of CGI.br are clearly mentioned (e.g., the academic community, the private sector, and the Internet user community). This ad-hoc set of stakeholders was empowered by working groups such as CT-Spam. This is the nature of the regulatory body that will address future challenges of Internet regulation in the country, such as in the case of net neutrality standards and IPv6 implementation.

Apart from that, we should also highlight an important observation from Brazil's blocking of Port 25/TCP experience: for Brazil it was important to ensure stakeholder support for solutions, which is a move beyond just identifying and implementing solutions with multiple stakeholders. 
CT-Spam focused on convincing stakeholders that they should implement a particular solution, which required several years of action.

Identifying the issue and finding a solution was still important. CT-Spam identified a policy issue almost by itself: few academics and CGI.br advisors were previously aware of the role of Brazil as an international spam source. They conducted research to confirm their thesis and liaised internationally to validate their technical solution. But the key step was convincing other stakeholders to support it, which proved to be a harder task than identifying the problem itself.

What the next seven years revealed was a complex implementation process that required stakeholders to believe in the multistakeholder decision-making process itself. This required Anatel to raise its role in CGI.br and consumer rights agencies to support the process and to defend telcos' and ISPs' interests. It also demanded that the Committee Board personally engage with decision makers from the private sector. At the end of the process, all those participating were satisfied with the implemented policy solution, and expressed willingness to support such initiatives in the future. While there are still disagreements around Internet regulation in Brazil, the CT-Spam case provided CGI.br with additional legitimacy. 


\section{Appendix E: \\ Enquete-Kommission Internet und digitale Gesellschaft (Enquete Commission on Internet and Digital Society)}




\title{
NoC Internet Governance Case Studies Series: Enquete-Kommission Internet und digitale Gesellschaft (Enquete Commission on Internet and Digital Society)
}

\author{
Kirsten Gollatz, Sarah Herweg, and Jeanette Hofmann ${ }^{1}$ \\ Humboldt Institute for Internet and Society
}

Editorial Note: Context, Character, and Purpose of the Case Study

This case study is part of a globally coordinated, independent academic research pilot project by the Global Network of Interdisciplinary Internet \& Society Research Centers (NoC). Facilitated by the Berkman Center for Internet \& Society at Harvard University, this study examines existing multistakeholder governance groups with the goal of informing the future evolution of the Internet governance ecosystem. Building upon the NETmundial Principles and Roadmap, it contributes to current policy debates at the international level, including the Internet Governance Forum, the NETmundial Initiative, and other organizations and efforts.

Internet governance is an increasingly complex concept that operates at multiple levels and in different dimensions, making it necessary to have a better understanding of both how multistakeholder governance groups operate and how they best achieve their goals. With this need in mind, at a point where the future of Internet governance is being re-envisioned, colleagues from several NoC institutions around the world have written twelve case studies examining a geographically and topically diverse set of local, national, and international governance models, components, and mechanisms from within and outside of the sphere of Internet governance. Key findings from these cases are summarized in a synthesis paper, which aims to deepen our understanding of the formation, operation, and critical success factors of governance groups and even challenge conventional thinking.

The research, based on twelve case studies, suggests that there is no single best-fit model for multistakeholder governance groups that can be applied in all situations. Rather, it reveals a range of approaches, mechanisms, and tools available for both the formation and operation of such groups. The analysis demonstrates that the success of governance groups depends to a large degree on the careful selection, deployment, and management of suitable instruments from this "toolbox." As governance groups pass through different phases of operation, conveners and facilitators must remain alert to changes in circumstances that necessitate adjustments to the approaches, mechanisms, and tools that they deploy in order to address evolving challenges from inside and outside. This case study provides insights into how those instruments can be deployed and adjusted over time within such groups, and highlights how their interactions with important contextual factors may be successfully managed within given resource restraints.

The research effort is grounded in a diversity of global perspectives and collaborative research techniques. Adhering to objective and independent academic standards, it aspires to be useful, actionable, and timely for policymakers and stakeholders. More broadly, the Network of Centers seeks to contribute to a more generalized vision and longer-term strategy for academia regarding its roles in research, facilitation and convening, and education in and communication about the Internet age.

For additional information on the initiative, please contact Urs Gasser, Berkman Center for Internet \& Society, at ugasser@cyber.law.harvard.edu.

\footnotetext{
${ }^{1}$ Kirsten Gollatz and Sarah Herweg wrote the first draft of this case study. The draft was partly based on interviews with Jeanette Hofmann and Wolfgang Schulz, both members of the former Enquete Commission. Jeanette Hofmann wrote the second draft and co-authored the third draft. This is why she is both co-author and source of this case-study.
} 


\begin{abstract}
This case study explores The Enquete Commission on Internet and digital Society (EIDG Commission), which is a parliamentary inquiry body of the German Bundestag that conducted its work from May 2010 until April 2013. Enquete Commissions are special bodies of the German Bundestag, which form an interface between policy-makers and academic and professional experts to examine through discussion forums and working groups broad and complex matters of society that cannot be sufficiently dealt with within the regular parliamentary framework. The Commission was introduced in response to rising awareness regarding the challenges for politics and society that digitization brings along, which became apparent in a controversial draft bill before the parliament that called for the mandatory blocking of websites with child pornography-related content. The Commission consisted of 34 individuals, 17 democratically elected members (members of parliament) and 17 experts and practitioners representing industry, trade unions, civil society and academia. Furthermore, the public was invited to participate in the Commission's consultations "to a special degree," which included efforts at transparency and an online engagement platform. It is too early to assess the policy impact of the Commission's work, as it is still unclear to what extent the new parliament will include the Commission's policy recommendations in its legislative initiatives. However, as a mechanism for bringing together heterogeneous sources of knowledge and expertise for the purpose of enlightening decision-making processes, the general model of an Enquete Commission can be regarded as an effective and legitimate mechanism for parliaments and possibly other deliberative bodies. Going forward, finding the appropriate implementation mechanisms that will help inform evidence-based policy making and provide justification for spending further resources should be taken into account in the process design.
\end{abstract}




\section{Table of Contents}

I. Introduction ..................................................................................................................................... 1

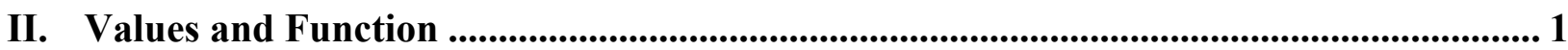

III. Organizational Model and Structure ................................................................................... 3

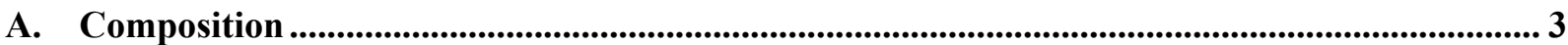

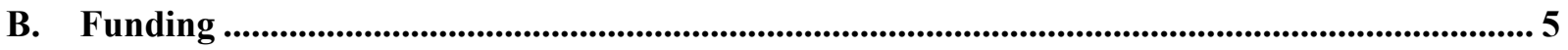

C. Membership structure.................................................................................................................................. 5

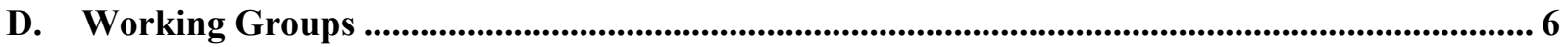

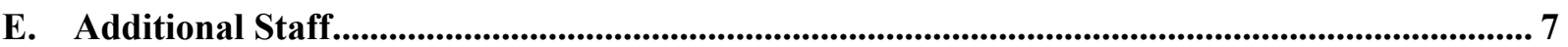

F. Other Stakeholders/ External Experts ................................................................................................ 7

G. Public Participation .............................................................................................................................. 7

H. Communication Between Members ........................................................................................ 10

IV. Decision-Making Structures............................................................................................. 10

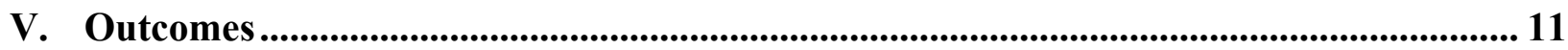

VI. Lessons Learned and Considerations for Future Organizations.................................... 12

A. Integrating Expertise and Knowledge into Politics in an Inclusive, Fair and Representative

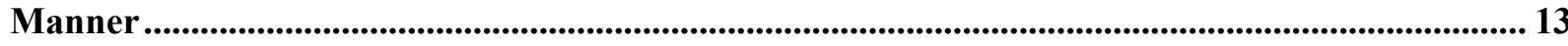

1. Integrating Knowledge Across Heterogeneous Domains .......................................................... 13

2. Identifying Future Policy Fields Beyond Current Controversies ................................................. 13

3. Providing Equal Status Among All Members ........................................................................... 14

4. Maintaining Independence/Autonomy of Experts in their Contributions .................................. 14

B. Deliberative Processes for Generating New Knowledge and Fostering Social Learning ....... 14

1. Democratizing Political Decision-Making Through the Generation of Knowledge ................... 14

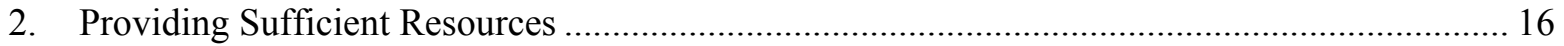

3. Compromising Between Degrees of Public Scrutiny .............................................................. 16

C. Translation Into Politics............................................................................................................................. 17 


\section{Introduction}

Enquete Commissions ${ }^{2}$ are an example of advisory institutions contributing to the representative tasks of the German Bundestag (Federal Parliament). They form an interface between policymakers, and academic and professional experts to examine and provide advice on emerging and complex matters that cannot be sufficiently dealt with within the regular institutional framework of the parliament and its committees. ${ }^{3}$ The term "enquete" has its roots in the Latin term "inquirere" [= inquire, investigate, examine]. ${ }^{4}$ As part of its parliamentary reform in 1969 , the German Bundestag established the instrument of an Enquete Commission in its Rules of Procedures. It was established as an instrument of information gathering to strengthen the role of the parliament vis à vis the government within the overall legislative institutional framework. ${ }^{5}$ There have been 27 Enquete Commissions since 1971 when the first of its kind was initiated. ${ }^{6}$ Enquete Commissions are relatively unique. Austria is the only other country that has introduced this parliamentary body.

Paragraph 56 of the Rules of Procedure of the German Bundestag provides for the establishment of an Enquete Commission for the purpose of investigating wide-ranging and significant issues by a motion of one quarter of its members. The motion resolution contains the Commission's mission and its terms of reference. ${ }^{7}$ The membership of Enquete Commissions consists, in equal numbers, of democratically elected members (members of parliament) and external experts, including representatives from the private sector, civil society, and the academic community. They are nominated by the parliamentary groups and appointed by the president of the German Bundestag. ${ }^{8}$ Enquete Commissions are thus parliamentary tools for addressing long-term societal challenges that exceed the expertise of the parliament but may also become the subject of public and academic controversies. They constitute a hybrid of a discussion forum and a working body, aiming to develop a basis for future decision-making around political challenges beyond day-today political affairs. Enquete Commissions are explicitly set up to overcome the constraints of the competition between political parties by bringing together academic experts and practitioners with political decision-makers. The administrative regulations of the German Bundestag determine the model (structure and institutional design) and limit the outputs of Enquete Commissions to general recommendations.

\section{Values and Function}

In general, Enquete Commissions are an instrument of the parliament to integrate expert advice

\footnotetext{
${ }^{2}$ The term "Enquete Commission" is translated either as parliamentary study or inquiry Commission. However, the specific set-up of the German Enquete Commission seems to be unique without any equivalents in other Western democracies.

${ }^{3}$ Other Enquete Commissions for example dealt with topics such as genetic engineering, demographic change, future education policy or law and ethics of modern medicine.

${ }^{4}$ Heyer Christian, and Stephan Liening. 2004. Enquete-Kommissionen des Deutschen Bundestags. Schnittstellen zwischen Politik und Wissenschaft, 2nd edition, Berlin, p. 7.

${ }^{5}$ Heyer and Liening 2004, p. 7f.

${ }^{6}$ See http://de.wikipedia.org/wiki/Liste_der_Enquete-Kommissionen_des_Deutschen_Bundestags (last visited 19.08.2014); Heyer and Liening 2004, p. 7f; http://daten.offenesparlament.de/datenhandbuch_19902010/08/08 10/index.html.

${ }^{7}$ German Bundestag. 2013. Rules of Procedure of the German Bundestag and Rules of Procedure of the Mediation Committee, Berlin, §56, p. 45. (Further cited as "Rules of Procedure")

${ }^{8}$ Heyer and Liening 2004, p. 12f.
} 
in legislative processes. They serve both problem-oriented functions and politics-oriented functions. ${ }^{9}$ The former directs attention to the aim of using expert knowledge to identify and understand the issue at stake, including alternative perspectives and available evidence related to the problem. The latter refers to the communicative and strategic use of expert advice in order to determine the political agenda, or to justify policies and political positions.

The Enquete-Kommission Internet und digitale Gesellschaft (EIDG) ${ }^{10}$ combined both functions. It was established through a resolution by the German Bundestag, proposed by four out of five parliamentary groups, and was adopted unanimously on March 4, 2010. ${ }^{11}$ In general, Enquete Commissions are regarded as a means for the opposition parties of the parliament to shape and set the political agenda. However, EIDG was the result of a joint effort by all parties and various industry associations. This unusual alliance was due to political controversies in 2009, a federal election year in Germany. The German minister of family affairs had put a draft bill before the parliament, which called for the mandatory blocking of websites with child pornography-related content. ${ }^{12}$ The law stipulated that all Internet service providers would sign a contract with the government forcing them to filter Internet content based on secret black lists compiled daily by the federal crime police office. The draft law caused a political outcry not only among civil rights organizations, but also across German stakeholder groups including parts of the Internet industry. Despite the massive opposition, the German federal parliament adopted the law in 2009. However, the widely shared argument that content filtering is the wrong approach to combating child pornography won in the end and the law was never enforced. ${ }^{13}$

What was regarded as a triumph among the Internet community looked more like a disaster from the perspective of the members of the parliament. The polarized public atmosphere in the aftermath of the access impediment act gave rise to the general belief that an Enquete Commission was needed to look at the development of the Internet and its regulatory implications in more depth. An Enquete Commission was also thought to have positive educational effects on the members of the parliament, many of which still rely on their staff for Internet communication.

The appointment resolution of EIDG specified its mission including the topics it should address. The ruling parties of the Bundestag produced the first draft of the appointment resolution, which placed emphasis on commercial aspects of the Internet. ${ }^{14}$ In the subsequent negotiations with the

\footnotetext{
${ }^{9}$ Mark B. Brown et al. 2005. Representation, Expertise and The German Parliament: A Comparison of Three Advisory Institutions, in: Democratization of Expertise? Exploring Novel Forms of Scientific Advice in Political Decision-Making edited by S. Maasen, and P. Weingart, P. Dordrecht: Springer, 2005, p. 81.

${ }^{10}$ English translation: Enquete Commission for Internet and Digital Society.

${ }^{11}$ The four parliamentary groups were the Christian Democratic Party (CDU/CSU), the Social Democratic Party (SPD), the Free Democratic Party (FDP) and the Green Party. The appointment resolution traces back to a proposal from the government coalition of CDU and FDP, $c f$. Krempl, Stefan. 2010. Schwarz-gelb sucht übergreifende Regeln fürs Internet. heise online, 14 January 2010, available at: http://www.heise.de/newsticker/meldung/SchwarzGelb-sucht-uebergreifende-Regeln-fuers-Internet-904684.html (last access 17 July 2014).

${ }^{12} \mathrm{http}: / /$ www.zugerschwg.com/.

${ }^{13}$ Due to the substantial criticism the Access Impediment law (Zugangserschwerungsgesetz) never came into effect and was finally repealed by the German parliament in December 2011, cf. Freedom House Foundation (eds.) Freedom on the Net Report 2012 - Germany, available at: http://freedomhouse.org/report/freedomnet/2012/germany\#.U_yLKDK00vI (last access 26 August 2014).

${ }^{14}$ The Christian Democratic Party formed a coalition government with the Liberal Democratic Party during the parliamentary term from 2009 until 2013. They also had the majority of members in the Enquete Commission (see
} 
opposition parties, ${ }^{15}$ civil rights-related topics were added to the agenda after opposition parties threatened to introduce their own version of an appointment resolution. Ultimately, the consensual appointment resolution covered a wide range of issues and reflected the concerns of all negotiating parties. ${ }^{16}$

The Internet Enquete commission took up its work in May 2010 and the German Bundestag adopted its final report in April 2013. The mission of EIDG as enshrined in the appointment resolution $^{17}$ was twofold: Members of the commission were called (1) to examine chances and challenges of digitization and (2) to develop recommendations for German legislators in six issue-areas:

- Culture and media

- Economy and environment

- Education and research

- Consumer protection

- Law and domestic policy

- Society and democracy

Furthermore, the parliament asked the EIDG to involve the public in its consultations "to a special degree," which involved informing the public as regularly and transparently as possible about its progress via the Bundestag website. ${ }^{18}$ The mission of EIDG also provided for the deployment of tools for online participation that would ensure the involvement of the public in the work and progress of the commission. Notably, the high degree of public participation that the parliament called for is not a general characteristic of Enquete Commissions. Rather, it reflected the subject matter of EIDG. Since the Internet facilitates reaching out to the broader public and allows for easier participation, the call for public consultation can be regarded as both a goal as well as an experiment.

\section{Organizational Model and Structure}

\section{A. Composition}

Most important for the work of an Enquete Commission are its members. Their expertise and experience in the respective field-be it as scientific experts, practitioners, or policy expertsprovides the commission with the necessary knowledge, and a broad range of perspectives and opinions to serve as the basis for developing policy recommendations. The EIDG consisted of 34 individuals, 17 democratically elected members (members of parliament) and 17 experts and practitioners representing to fairly equal parts industry, trade unions, civil society and academia. Amongst the experts and practitioners were IT-entrepreneurs, lawyers, bloggers, programmers,

\footnotetext{
the chart below).

15 The Social Democratic Party, the Green Party and the Left formed the opposition in the federal parliament. The Left did not participate in the negotiations but supported its outcome.

${ }^{16}$ Information by a member of staff of the Social Democratic Group of the German Bundestag.

${ }^{17}$ Deutscher Bundestag. 2010. Antrag der Fraktionen CDU/CSU, SPD, FDP und Bündnis 90/ Die Grünen. Einsetzung einer Enquete-Kommission "Internet und digitale Gesellschaft”. Bundestagsdrucksache 17/950, 3 March 2010, p. 2. (Further cited as “Appointment Resolution”).

${ }^{18}$ Appointment Resolution, p. 4.
} 
consumer advocates, and researchers. ${ }^{19}$ The parliamentary groups were responsible for the nomination of the external expert members. Following the Rules of Procedures, the composition reflected the relative size of the parliamentary groups as represented in the German Bundestag (see Figure 1). In this sense, proportional political representation is a very implicit goal of the commission's institutional design. ${ }^{20}$

In contrast, it is worth noting that the nomination procedure of social and disciplinary representatives did not follow a transparent process and differed for every parliamentary group. ${ }^{21}$ While the external experts were chosen according to their expertise, political leanings also played an important role in doing so. In general, parliamentary groups are unlikely to appoint experts that do not share and support their party's political stance. ${ }^{22}$ Thus, the conservative Christian Democrats (CDU/CSU), for instance, appointed representatives of the industry and lawyers that would advocate for more conservative political viewpoints, while the Left Party appointed a trade union representative and the spokesperson of a traditionally leftist hacker club.

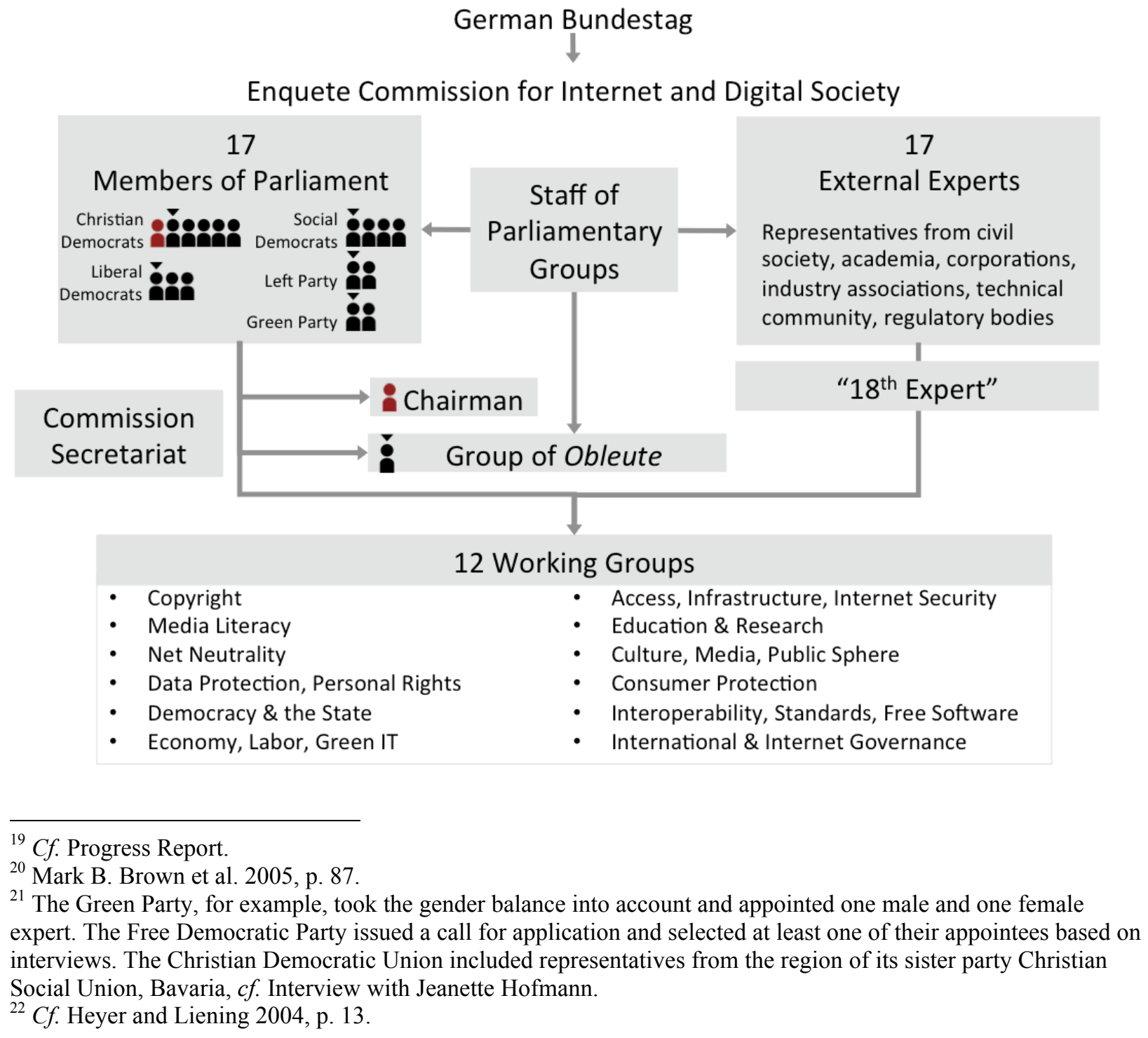


Figure 1. Enquete Commission for Internet and Digital Society of the German Bundestag: Roles, Resources and Relations

\section{B. Funding}

Being a parliamentary forum, the EIDG was financed by the German Bundestag. Its meetings were held in the conference rooms of the parliament. The expert members received a modest ${ }^{23}$ expense allowance for their work. However, it is unlikely that this allowance served as an incentive for participating in the EIDG. Rather, expert members participated because of their general interest in the topic, the possibility to shape the future policy agenda of the parliament, and perhaps prestige. ${ }^{24}$ Besides the funding of expense allowances and running costs, no further budget was allocated for the Enquete Commission.

\section{Membership structure}

It is the underlying idea of Enquete Commissions that democratically elected members and appointed expert members should work together on equal footing. Accordingly, there were no formal distinctions between democratically elected and appointed members. Only the chair of the EIDG, the chairs of the working groups, and the Obleute (parliamentary spokespersons) can be said to have a more dominant role. However, since the composition of the EIDG reproduced the parliament's political balance of power, the governing Christian Democrats and Liberals had more democratically elected as well as expert members, and thus most of the time held the majority of votes. ${ }^{25}$ Also, the resources at the disposition of the individual members differed enormously. ${ }^{26}$

The largest parliamentary group (Christian Democrats, CDU) also nominated the chair of the EIDG who was elected in the constituent meeting. The chair, who has to be a member of the German parliament, ${ }^{27}$ is responsible for preparing, convening, and conducting plenary meetings. ${ }^{28}$ (See Figure 1)

Every parliamentary group also chooses an Obmann or Obfrau [plural: Obleute] to act as spokesperson and - in case of conflict-as chief negotiator of the respective parliamentary group. Together with the Commission chair, the Obleute deliberate on points of order and other technical issues; they are furthermore responsible for selecting the experts to be invited for hearings. ${ }^{29}$ In practice, the Obleute are almost always democratically elected members, thus members of parliament. As a result, there is a risk of experts playing a subordinate role with regard to the procedural aspects of an Enquete Commission. However, decisions by the Obleute

\footnotetext{
${ }^{23}$ Given the considerable workload particularly for the expert members.

${ }^{24} C f$. Interviews with Jeanette Hofmann and Wolfgang Schulz.

${ }^{25}$ The impact of the imbalance in the membership composition first became obvious when the number of questions in expert hearings where allocated according to the number of EIDG members of each parliamentary group. Later on, this procedure was replaced by rounds of questions, $c f$. Interviews with Jeanette Hofmann and Wolfgang Schulz. ${ }^{26}$ One of the trade union representatives, for example, had three employees dedicated to EIDG work at his disposal, which enabled him to contribute disproportional amounts of texts, $c f$. interview with Wolfgang Schulz.

${ }^{27}$ The chair executes domiciliary rights in place of the president of the Bundestag in accordance with $\S 40$, subsection 2, first sentence of the German Constitution; Heyer and Liening 2004, p. 14.

${ }^{28}$ Unlike the president of the German Bundestag who does not participate in debates of the parliament, the chair of an Enquete Commission is allowed to contribute, $c f$. Heyer and Liening 2004, p. 14.

${ }^{29}$ Cf. Heyer and Liening 2004, p. 15; Interview with Jeanette Hofmann.
} 
are only preliminary and are hence open to debate in the Commission plenary. ${ }^{30}$

\section{Working Groups}

While the EIDG held 20 plenary meetings, the main work took place in working groups. ${ }^{31}$ The working groups produced the reports that the EIDG plenary later discussed and voted upon. While original plans provided for three permanent working groups covering "law," "economy," and "politics," a majority of EIDG members argued for a different approach and decided during their Conclave in May 2010 to set up twelve working groups. ${ }^{32}$ Their topics and dates of commencement are displayed in the following chart, which shows the order of events from the genesis to the termination of the Internet-Enquete.

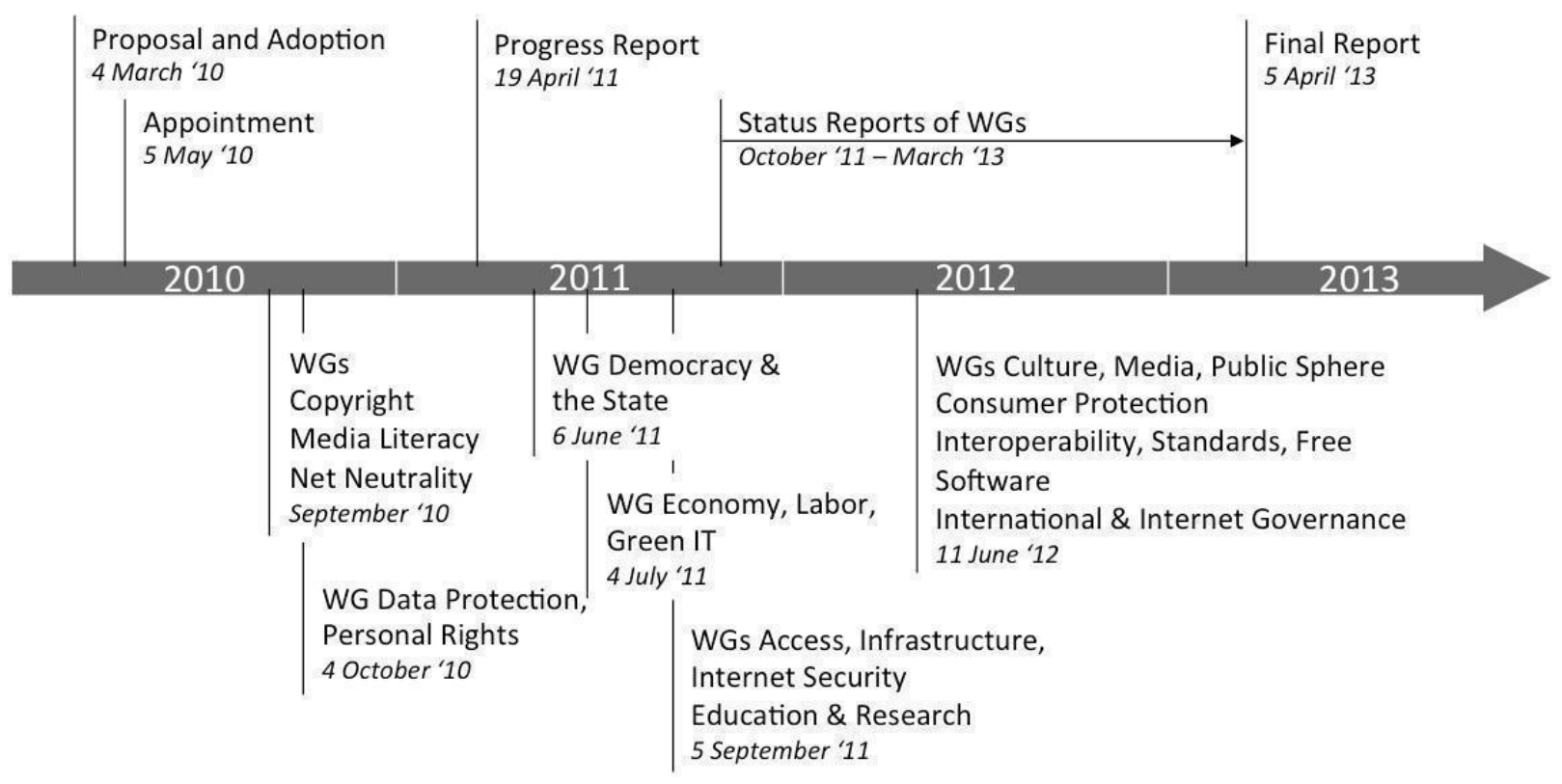

Figure 2. Enquete Commission for Internet and Digital Society of the German Bundestag: Timeline of events from 2010 until 2013

Each working group was comprised of nine voting members appointed by the parliamentary groups. These members had the right to vote on preliminary reports. However, working groups were open to contributions by interested EIDG members, which is why some working groups had up to 26 members. ${ }^{33}$ Chairs and co-chairs were chosen by the Obleute. While the EIDG had

\footnotetext{
${ }^{30} C f$. Heyer und Liening, p. 16.

${ }^{31}$ Cf. Statement Dr. Wolf Osthues in Final Report, p. 19f.

${ }^{32} C f$. Interview with Jeanette Hofmann; Progress Report, p. 3.

${ }^{33}$ Lists of members of each working group can be found at http://webarchiv.bundestag.de/cgi/show.php?fileToLoad=2944\&id=1223. Noticeably, the first working groups were the most popular ones in terms of contributing members (e.g. the working groups on copyright and net neutrality). The working groups starting late 2011 were less well attended due to their significant workload. Enquete Commissions usually are a heavy burden both for the expert members with their individual jobs and the parliamentary members who have tight schedules, especially during session weeks. There was therefore the tacit expectation that a substantial part of committee work was to be done by the expert members, $c f$. Interview with Jeanette Hofmann.
} 
to establish several working groups in parallel, meetings were almost never held at the same time to allow members to attend. The first working groups covered the topics of net neutrality, copyright, privacy and personal rights, and media literacy. ${ }^{34}$

\section{E. Additional Staff}

The EIDG secretariat, which consisted of up to 15 employees, supported the meetings of the plenary and, more importantly, the writing of reports of each working group. More specifically, the secretariat staff was responsible for taking notes and writing minutes as well as for compiling the contributions that were discussed during working group meetings. Furthermore, the secretariat supported the chair in managing the EIDG. In addition to the Enquete secretariat, all parliamentary groups hired at least one staff person to support their members in producing draft documents and to facilitate communication among the group. ${ }^{35}$

\section{F. Other Stakeholders/ External Experts}

Besides the appointed expert members as sources of information and expertise, another venue for information gathering of Enquete Commissions are expert hearings. As the appointed expert members' areas of competence might not cover all subject matters of the Enquete Commissions' mission, further experts can be invited to contribute additional knowledge. ${ }^{36}$ In the case of EIDG, almost every working group conducted public hearings. The EIDG members agreed on a set of questions for the invited experts, and their written answers formed the basis for the public hearing. All in all, EIDG conducted 13 expert hearings with roughly 85 external experts between 2010 and 2013. External experts included public servants, academics, practitioners, industry, and civil society representatives. ${ }^{37}$ Not least of all, public hearings serve as a means to spur public interest and involve the interested public in the commission's work.

\section{G. Public Participation}

The mission of the EIDG stipulated involving the public "to a special degree." 38 Hence, questions of transparency and participation were given center stage from the beginning. Contrary to the principle laid down in the Rules of Procedure that Enquete Commissions do not work publicly, ${ }^{39}$ the EIDG decided to make its plenary meetings publicly available through live streaming unless otherwise decided. ${ }^{40}$ Some of the working groups moreover decided to make their meetings public, although these latter decisions were controversial. One concern was that transparency would politicize the working groups; another concern was that their members would lose the chance to change their opinion without having to declare them publicly. ${ }^{41}$ In practice, the parliament lacked the technical and financial resources to stream all working group

\footnotetext{
${ }^{34}$ Progress Report; Deutscher Bundestag. 2010. Enquete Kommission Internet und digitale Gesellschaft:

Kurzprotokoll der 3. Sitzung, Protokoll Nr. 17/3, Berlin, June 14, 2010.

${ }^{35} \mathrm{Cf}$. Interview with Jeanette Hofmann.

${ }^{36}$ Heyer and Liening 2004, p. $18 f$.

${ }^{37}$ For specific information on invited experts $c f$. the collection of protocols and minutes, http://webarchiv.bundestag.de/cgi/show.php?fileToLoad=2944\&id=1223.

${ }^{38}$ Appointment Resolution, p. 4.

${ }^{39}$ As set up in the Rules of Procedure, $\$ 69$.

${ }^{40}$ Cf. Interview with Jeanette Hofmann; Deutscher Bundestag. 2013. Schlussbericht der Enquete-Kommission "Internet und digitale Gesellschaft". Bundestagsdrucksache 17/12550, 5 April 2013, p. 7. (Further cited as "Final Report").

${ }^{41}$ Cf. Final Report, p. 7f.; Statement Prof. Dr. Christof Weinhardt in Final Report, p. 23.
} 
meetings. As a compromise, expert hearings held by working groups were streamed online and were open for the public to attend. ${ }^{42}$ Although exact numbers of live stream viewers do not exist, we know that the live stream of an expert hearing in July 2010, for example, had 1833 views with an average duration of 26 minutes. Afterwards, the video was uploaded on the website and was watched 366 times within the first 20 hours. ${ }^{43}$

The EIDG also included the public in the form of the so-called "18th expert" (18. Sachverständiger). The 18th expert played a symbolic role and more or less boiled down to a speaking slot for a remote participant at EIDG plenary sessions and hearings. This speaking slot enabled observers to address invited experts with comments or questions, for instance. ${ }^{44}$

As already suggested in the mission, the EIDG commissioned a microsite ${ }^{45}$ that had its own navigation and allowed for a new degree of public involvement well surpassing the usual practice in parliamentary commissions. ${ }^{46}$ It contained agendas, meeting minutes, and reports on every public or non-public meeting in addition to a calendar with all public meetings, announcements, summaries and calls for participation. ${ }^{47}$ The EIDG also established a blog for its members to communicate their personal impressions of the commission's work to the public. The blog included a public forum enabling observers to ask questions and discuss topics with the commission members directly. ${ }^{48}$

Besides the speaking slot for the 18th expert and the microsite, public consultation was encouraged predominantly through the participatory online platform enquetebeteiligung.de, ${ }^{49}$ which enabled public participation throughout the different phases of working group consultations. This entailed the possibility of commenting and revising drafts, as well as suggesting additional text. The working groups decided individually how and to what extent to incorporate contributions thus made by the public. Some working groups adopted public recommendations - sometimes literally-for their preliminary report, while others listed the public recommendations in their preliminary reports to justify the decision to adopt them or to reject them, while yet others included them in their preliminary reports as dissenting opinion. ${ }^{50}$

Enquetebeteiligung.de went online in February 2011, with considerable delay. The delay was mainly due to the adverse reaction of the Federal parliament administration. As there was no budget for EIDG, the Bundestag presidency was not willing to finance the development and implementation of the EIDG participatory tool. ${ }^{51}$ Adhocracy, the open source platform used for

\footnotetext{
${ }^{42}$ Cf. Final Report, p. 7.

${ }^{43}$ Beckedahl, Markus. 2010. Statistiken zur Enquete-Anhörung. Netzpolitik.org, 7 July 2010, https://netzpolitik.org/2010/statistiken-zur-enquete-anhoerung/.

${ }^{44}$ Freude, Alvar C.H. 2011. Die Online-Beteiligung kann endlich starten! Blog der Enquete-Kommission Internet und digitale Gesellschaft. February 25, 2011.

http://webarchiv.bundestag.de/cgi/show.php?fileToLoad=3414\&id=1223.

${ }_{45}$ Accessible through www.bundestag.de/internetenquete.

${ }^{46}$ Cf. Final Report, p. 7f.

${ }^{47}$ Deutscher Bundestag. 2011. Zwischenbericht der Enquete-Kommission "Internet und digitale Gesellschaft". Bundestagsdrucksache 17/5625, 19 April 2011, p. 4. (Further cited as "Progress Report"); Final Report, p. 7.

${ }^{48}$ The forum was later supplemented by the participatory platform www.enquetebeteiligung.de, $c f$. Progress Report, p. 4.

${ }^{49}$ Accesible via www.enquetebeteiligung.de.

${ }^{50}$ Cf. Final Report, p. 11.

${ }^{51} C f$. Interview with Jeanette Hofmann.
} 
the site, is a product developed by the association Liquid Democracy e.V., ${ }^{52}$ which aims to enhance democratic participation by means of software tools. As a consequence of the opinion of the parliament administration, the association built the EIDG website without getting remuneration. Not only the implementation of the participation platform was controversial, the tool remained a controversial topic among the members of the Enquete Commission as well. ${ }^{53}$

Assessing the success of public involvement, the transparency of and public participation in the EIDG's work was unparalleled compared to other Enquete Commissions, though there is clearly room for improvement. The implementation of the online-participation tool enquetebeteiligung.de particularly suffered from a sort of culture clash between the Internet community and the bureaucracy of the Federal parliament. This culture clash might be a result of the unfamiliarity of the administration with participatory online tools and the resulting unwillingness for funding. Thus in practice, although participants expressed satisfaction with the adhocracy platform, ${ }^{54}$ its implementation took a long time and consequently came too late to be fully accepted and integrated into the EIDG's working procedure. ${ }^{55}$

In terms of numbers, the participation platform had 30,000 visitors and 3,250 active participants who performed 80,000 individual actions such as commenting or uploading text. The number of text proposals for working groups lay between nine (international \& Internet governance) and 81 (democracy and the state). ${ }^{56}$ However, participation in votes on proposals (a specific feature of the adhocracy platform) was only in the single- or double-digits. While the quality of the contributions was generally regarded as high, the extent of participation was seen as disappointing by many. ${ }^{57}$ On the other hand, a study conducted by Zeppelin University found out that users were very content with the tool and regarded their participation as meaningful although they did not believe to have a major influence on the work of EIDG. As is the case with most forms of online participation, the group of users of enquetebeteiligung.de was a very specific demographic: users were mainly males that were interested in politics and mostly older than 40 years. $^{58}$

Notwithstanding the overall support for the experiment of involving the public, ${ }^{59}$ it was a major challenge for the EIDG to link public contributions to its own contributions. For some members it just meant an extra workload to include the public contributions into the working group reports, especially since there were neither established guidelines nor allocated time frames for doing so. ${ }^{60}$ Overall, the openness of EIDG members to incorporate contributions from the onlineplatform varied.

\footnotetext{
${ }^{52}$ Accessible via https://liqd.net/en/.

${ }^{53} \mathrm{Cf}$. Interview with Jeanette Hofmann.

${ }^{54}$ Große et. al. 2012. Der Erfolg von enquetebeteiligung.de. Begleitforschung zur Adhocracy-Plattform der EnqueteKommission "Internet und digitale Gesellschaft”. Version 1.0. Deutsches Telekom Institut für Connected Cities,

Zeppelin Universität, Friedrichshafen. Available online at:

https://fold.liqd.net/netzwerk/forschungsprojekte/zeppelin-university/.

55 The website enquetebeteiligung.de was launched in 2011 whilst the Enquete Commission began working in 2010.

The first four working groups introduced online participation only at an advanced stage. $C f$. Interview with

Wolfgang Schulz.

${ }^{56}$ Cf. Final Report, p. 9 and 11.

${ }^{57}$ Cf. Final Report, p. 9 and 11.

${ }^{58}$ Große et al. 2012.

${ }^{59}$ Cf. Große et al. 2012.

${ }^{60} C f$. Final Report, p. $12 ; c f$. Interview with Jeanette Hofmann.
} 


\section{H. Communication Between Members}

Communication between members took place predominantly during the EIDG meetings themselves. Working group members also used mailing lists as an important channel for circulating new drafts and organizing the work. ${ }^{61}$ However, pre-existing relationships between EIDG members also played a considerable role. Relationships outside the realm of EIDG included professional relationships (e.g. between the participating law experts) as well as advocacy connections between four expert members who participated in the World Summit on the Information Society (WSIS) and its follow-up meetings. ${ }^{62}$ Informal communication across parliamentary groups was also important at times, particularly towards the end of a working group when such informal communication was used to do a number of things, such as form majorities for certain recommendations. ${ }^{63}$

\section{Decision-Making Structures}

The basic rules for participating and voting in Enquete Commissions are specified in the Rules of Procedure of the German Bundestag. However, every Enquete Commission may add its own rules and specifics. In the case of EIDG, these included the decision whether or not meetings should be held in public, the number of working groups, the implementation of an online participation tool, and that there should be a Wifi connection available during plenary and working group meetings.

In the EIDG, as in other Enquete Commissions, the degree of politicization increased before and during voting over working group reports. The working groups of EIDG generally produced their reports on a consensual basis. ${ }^{64}$ This was particularly true for the first parts of the reports, which consisted of an assessment of the topics to be discussed. If conflicts arose in the working groups, they typically concerned the second part of the reports which included the conclusions and recommendations based on the preceding assessments. These conflicts mostly arose over issues that touched upon current political affairs and were therefore subject to political debate in the press and by the parliament. Thus, the line of division in the Enquete Commission usually ran between the ruling coalition group and their experts on the one hand, and the opposition groups and the respective experts. In cases of conflict, a majority vote of the EIDG plenary (needed to approve all working group reports) would decide over the text to be included in the report. Such plenary debates often resulted in crucial votes ("Kampfabstimmungen" ${ }^{65}$ ) so that EIDG members had to vote sentence-by-sentence on working group reports. ${ }^{66}$ Dissenting opinions would also be included. Depending on their length, they would either be included in a footnote on the same page or in an annex. ${ }^{67}$

Although formally independent, many expert members were expected to vote in accordance with the parties that had appointed them. This was particularly true for expert members appointed by the governing coalition (Christian Democrats, Liberals). Various expert members criticized the

\footnotetext{
${ }^{61} C f$. Interview with Jeanette Hofmann.

62 The four expert members with WSIS background were Annette Mühlberg, Markus Beckedahl, Alvar Freude and Jeanette Hofmann.

${ }^{63} \mathrm{Cf}$. Interview with Jeanette Hofmann.

${ }^{64} C f$. Interview with Wolfgang Schulz.

${ }^{65} C f$. Interview with Wolfgang Schulz.

${ }^{66} C f$. Interview with Jeanette Hofmann.

${ }^{67} C f$. Interview with Jeanette Hofmann.
} 
political pressure exerted on them as this was perceived to run counter to the mission and principles of an Enquete Commission. ${ }^{68}$ Expert members of the opposition parliamentary groups enjoyed considerably more freedom and in some cases led groups suggesting to others how to vote on specific reports. ${ }^{69}$ An expert member appointed by the Liberal Party negotiated his dissenting votes on an individual basis. ${ }^{70}$

\section{Outcomes}

On 18 April 2013, after almost three years of work, the German Bundestag acknowledged the Enquete Commission's work by adopting its final report. ${ }^{71}$ The output of the Commission was comprised of twelve reports from the twelve working groups, one progress report and one final report including an evaluation of transparency and public participation, as well as retrospective observations by all 17 expert members. All reports together contained several hundred recommendations on more than 1300 pages. Many EIDG members later expressed some regret about the EIDG's overly broad focus. More profound results could have been achieved if the Enquete Commission had focused on a few core points rather than trying to cover all aspects surrounding the issue. ${ }^{72}$ The evaluation of the reports, and thus the outcome of the EIDG, therefore has to take into account their double function. The first task of the reports was to assemble all relevant information on a given topic, describe the status quo, and do a deficit analysis (assessment report). The second task was to develop policy recommendations. Because Enquete Commissions in general comprise of non-elected members, the Bundestag's administrative regulations limit these recommendations to general, instead of specific, policy advice.

Most of the twelve assessment reports were detailed and very comprehensive. Different viewpoints by EIDG members were included in the form of neutral descriptions so that readers were given the opportunity to form their own opinion on a given issue. ${ }^{73}$ Achieving consensus on policy recommendations was the more difficult task. As noted above, votes were often aligned along partisan lines and therefore reflect the power balance of the German parliament, rather than the wisdom of EIDG's expertise. The majority voting concerning policy recommendations has general repercussions for the legitimacy of an Enquete Commission. Ideally, an Enquete Commission should ground its judgments and recommendations primarily in its broad

\footnotetext{
${ }^{68} C f$. Interviews with Jeanette Hofmann and Wolfgang Schulz; for opinions of other expert members, $c f$. among others: Statement by Prof. Dr. Hubertus Gersdorf in Final Report, p. 26; Statement by Annette Mühlberg in Final Report, p. 27; Statement Alvar Freude in Final Report, p. 28f., Statement by padeluun in Final Report, p. 31.

${ }^{69}$ Wolfgang Schulz, who was appointed by the Social Democrats, had such a role, $c f$. Interview with Wolfgang Schulz.

${ }^{70} C f$. Interview with Jeanette Hofmann.

${ }^{71}$ Originally having planned to conclude in summer 2012, the German Bundestag extended the term of the EIDG until the end of the year 2012 as it had become obvious that the Commission would not be able to complete its work in the originally time frame due to the breadth and complexity of the topic, $c f$. Deutscher Bundestag. 2012. Antrag der Fraktionen der CDU/CSU und FDP. Verlängerung der Arbeit der Enquete-Kommission "Internet und digitale Gesellschaft". Bundestagsdrucksache 17/9939, June 12, 2012.

http://dipbt.bundestag.de/extrakt/ba/WP17/246/24667.html.

${ }^{72} C f$. Interview with Wolfgang Schulz; Statement by Annette Mühlberg in Final Report, p. 27; Statement by Markus Beckedahl in Final Report, p. 35.

${ }^{73} C f$. Interview with Jeanette Hofmann; $C f$. Statement Dr. Wolf Osthues in Final Report, p. 19.
} 
knowledge base; however, the reports mirror first and foremost the strength of the parliamentary groups. $^{74}$

It is too early to assess the policy impact of the EIDG's work. Since the Enquete Commission finished its work roughly six months before the federal elections, it is still unclear to what extent the new parliament will include the EIDG's policy recommendations in its legislative initiatives. An obvious impact of the EIDG concerns is the increased relevance of Internet policies. Evidence of its political resonance can be found both in the new government program, which for the first time includes a "digital agenda," and the constitution of a new permanent parliamentary committee concerning itself with the Digital Agenda. ${ }^{75}$ The establishment of a parliamentary committee on Internet policies was a key recommendation of the EIDG. ${ }^{76}$

Having given center stage to Internet policy for the first time, the EIDG has furthermore increased public resonance. Their work led to a professionalization of, and further specialization within the German Internet community. Media coverage of this domain has also been greatly improved. The Commission's work additionally brought to light linkages between different subthemes of Internet policy. ${ }^{77}$

\section{Lessons Learned and Considerations for Future Organizations}

An evaluation of the strengths and weaknesses of the Internet Enquete inevitably leads to ambivalent results. ${ }^{78}$ The question is whether the Enquete Commission can be a model for gathering expertise and channeling it into decision-making bodies such as parliaments, regulatory agencies, or other multi-stakeholder bodies? We think some insights for other policy contexts can be gained from this case.

A crucial element for the work of Enquete Commissions is that they operate within the regulated framework provided by the rules of procedures of the German federal and state parliaments. These frameworks are both enablers and constraints; while they guarantee the necessary resources and the stability of the process, they also tie the work of the Enquete Commissions to the rationalities and dynamics of the parliamentary process. This ambivalent effect of the parliamentary framework also concerns the legitimacy of Enquete Commissions. As codified bodies of the parliament, Enquete Commissions enjoy an excellent reputation and the expertise involved has the potential to strengthen the legitimacy of policy programs based on its recommendations. At the same time, the Commission's integration in the institutional structure and processes of the parliament represents a source of potential de-legitimization. This is particularly evident for the majority voting on working group reports. While the working groups

\footnotetext{
${ }^{74} \mathrm{Cf}$. Interview with Jeanette Hofmann.

${ }^{75}$ The website of the permanent Commission Digitale Agenda is available at: http://www.bundestag.de/bundestag/ausschuesse18/a23.

${ }^{76}$ Jessica Binsch, “Enquete-Abschlussbericht. Netzpolitiker fordern Internet-Minister,” Spiegel Online, April 18, 2013. Last accessed July 18, 2014. Available online at: http://www.spiegel.de/netzwelt/netzpolitik/internet-enquetefordert-ausschuss-und-minister-a-895256.html.

${ }^{77} \mathrm{Cf}$. Interview with Jeanette Hofmann.

${ }^{78}$ There is no established mechanism that would enable Enquete Commissions to learn from each other and pass on lessons to future Enquete Commissions. Likewise, we do not know of any attempts to extract "take aways" from an Enquete Commission for other multistakeholder processes.
} 
were generally characterized by a spirit of fact finding and deliberation, the plenary meetings lacked this explorative, academic attitude and focused on the securing of a majority votes instead. This inherent hybrid between expertise-based and political power-based approaches resulted at times in feelings of unease among the expert members. This aspect also creates tensions in the architectural structure of Enquete Commissions per se and it may thus undermine the overall legitimacy of such bodies.

In our opinion, the aim of Enquete Commissions to gather high-level expertise on complex matters for future policy processes can be helpful in non-parliamentary contexts and should thus be transferable to transnational governance arrangements. To gain legitimacy, multi-stakeholder commissions modeled after German Enquete Commissions would thus have to secure sufficient resources and to create a reliable framework based on rules and procedures. Starting from this observation one can draw on related social mechanisms "that together constitute the core of most approaches to democratic governance beyond the state: inclusiveness, transparency, accountability, and deliberation." 79 In the following, we adopt these criteria as an analytical frame of reference in order to identify best practices and take-aways from the successes and failures of the Internet Enquete by looking more closely at its composition, its applied processes, and finally at activities for translating its outcome into politics.

\section{A. Integrating Expertise and Knowledge into Politics in an Inclusive, Fair and Representative Manner}

\section{Integrating Knowledge Across Heterogeneous Domains}

Given its mission to bring together heterogeneous sources of knowledge and expertise for the purpose of enlightening decision-making processes, the general model of an Enquete Commission can be regarded as an effective mechanism for parliaments and possibly beyond. Without a doubt, the Internet Enquete provided a substantial grounding for the Internet policy field in Germany. Pooling information that reflected multiple disciplinary perspectives and professional experiences in one parliamentary body eventually led to a knowledge corpus that enables readers to grasp the Internet-society interface in its multiple facets. The output of the Enquete benefited from the great variety of experts among its membership, and also from the additional expertise integrated through hearings and public participation.

\section{Identifying Future Policy Fields Beyond Current Controversies}

This point goes back to the notion of the complexity of the issues that have shaped the work of the Internet Enquete. The combination of politicians, professionals, and academic experts proved to be most successful for those issues that had not yet been part of political controversies. The chances for success of a multi-stakeholder model for knowledge acquisition therefore increases with the distance of a given issue from actual management tasks or decision-making processes. This may sound as if Enquete Commissions are practically irrelevant for imminent policy challenges; this, however, is not the case. The experience of the Internet Enquete rather suggests that timing is crucial for the probability of useful outcomes. In order to be successful, Enquete Commissions should be established before governance issues have evoked ideological controversies. Enquete Commissions work best if all participants share the interest in

\footnotetext{
${ }^{79}$ Marianne Beisheim/Klaus Dingwerth, "Procedural Legitimacy and Private Transnational Governance," SFBGovernance Working Paper Series No. 14, June 2008, 12.
} 
understanding a complex matter and do not feel the need to follow pre-defined or anticipated party lines in how they go about this task.

\section{3. $\quad$ Providing Equal Status Among All Members}

Equal footing among all members is one of the constitutive principles of Enquete Commissions. However, the composition of its membership, as well as the political pressure on the voting behavior of expert members, does not do justice to this principle. ${ }^{80}$ Although the Enquete Commission integrates external expertise into the process of political decision-making, it is designed in a way that clearly constrains the range of potential outcomes. Majority voting as practiced by Enquete Commissions is not adequate to come to agreements on policy recommendations. Instead of majority voting, other methods of consensus building should therefore be adopted for multi-stakeholder governance models. Voting should be replaced by deliberative procedures that consider all possible solutions to a given problem and assess them, for example, by means of cost-benefit analyses. In case of irreconcilable differences in opinion, Enquete Commissions should furthermore refrain from issuing policy recommendations and rather document their conflicting views as neutrally as possible. Such an evidence-based approach seems more suitable to multi-stakeholder environments that lack the legitimacy and representativeness of democratic constitutions. Expertise and sound processes of weighing different policy options can be an alternative source of legitimacy for transnational policy processes.

\section{Maintaining Independence/Autonomy of Experts in their Contributions}

The example of the Internet Enquete has illustrated a variety of relationships between appointed experts and the parliamentary groups that had nominated them. Although formally independent, expert members appointed by the governing parties were expected to vote according to political "imperatives." While this may seem an extreme case, the interaction between (academic) expertise and the realm of political decision-making generally affects both parties, as many other studies show. While experts run the risk of becoming associated with specific political interests to the detriment of their reputation and the perceived quality of their work, decision-makers may lose credibility and legitimacy if they are suspected of soliciting allegedly independent but politically suitable expertise. Setting up an Enquete Commission may thus backfire for all parties involved if the rules that govern the cooperation are inconsistent or not properly implemented.

\section{B. Deliberative Processes for Generating New Knowledge and Fostering Social Learning}

\section{Democratizing Political Decision Making Through the Generation of Knowledge}

Public reflection on specific subject matters by means of assembling many experts, competencies, and perspectives facilitates the creation of a knowledge base, which can be considered the foundation for informed decision-making. In fact, spelling out various decisionmaking options, including their potential downsides and benefits, constitutes a prerequisite for democratic processes. All available information about a political subject matter should be arranged in such a way that the pros and cons are comprehensible to people involved or affected by subsequent decisions to enable meaningful participation and strengthen the democratic right

\footnotetext{
${ }^{80}$ Certain elements in the rules of procedure extend the imbalance between democratically elected and appointed members. For example, members of parliaments can be substituted by other MPs while expert members cannot. Also, the central role of the chairman is filled with a member of parliament representing the largest political group.
} 
of freedom of expression. Enquete Commissions are a powerful instrument to facilitate such processes.

It is probably fair to say that all members of the Internet Enquete and many of its observers substantially broadened their horizons and acquired considerable factual knowledge. The effort of coordinating dissonant policy perspectives and thereby questioning one's own common wisdom has brought to the forefront policy options previously only considered by a minority of experts. Public hearings contributed significantly to this and in many ways moved relevant Internet-related expertise closer to the center of political decision-making.

The experience of the Internet Enquete shows, however, that the variety of perspectives and resulting recommendations loses addressees' respect and appreciation when an Enquete touches on issues relevant to the day-to-day affairs of the parliament. More generally, opening up the process of knowledge creation is more likely to succeed for issue domains that are considered less relevant to current political affairs. Thematic working groups - the fair treatment of all perspectives and open debate on alternative policy options-became politicized later on in the Internet Enquete by tightly controlled majority voting. Put simply, the Internet Enquete saw two different logics and legitimacy concerns at work: the logic of gathering and integrating knowledge to enhance the quality of the Enquete's output on one hand, and the logic of supporting and justifying certain political principles and courses of action on the other hand. The need to agree on political recommendations but also the increasing strategic political importance ascribed to Internet policies throughout its term made these logics clash and at times caused frustration among all parties involved.

The concept of deliberative democracy is based on the idea that the informed public discourse can legitimize political decision-making. The advocates of deliberative democracy highlight various democratic functions of public discourses, i.e. agenda setting, the identification of problems and the need for action, assessing the adequacy of political measures, and holding decision-makers to account. However, democratic procedures such as voting and parliamentary forms of representation often do not work outside of constitutional spaces; the question arises whether specific elements of deliberative democracy can help to compensate for these inherent democratic deficits in non-constitutional spaces.

Addressing problems of legitimacy and accountability in transnational and global governance, actors in global politics are seeking to institutionalize rules and practices for knowledge production and validation as a source of legitimate power in global decision-making. Following Clark A. Miller, this has resulted in the creation of international knowledge institutions, which "offer an opportunity to structure global politics so that it is determined not by the will of the most powerful but rather the outcomes of broad processes of deliberation informed by knowledge and reason." 81

Distributed governance bodies modeled after or drawing on aspects of Enquete Commissions might provide a path towards building democratic legitimacy in transnational governance settings. Transnational publics could be ideal laboratories for experimenting with approaches of deliberative democracy because they represent a unique combination of geopolitical and

\footnotetext{
${ }^{81}$ Clark A. Miller, "Democratization, International Knowledge Institutions, and Global Governance," Governance: An International Journal of Policy, Administration, and Institutions vol. 20(2) (April 2007): pp. 325-357.
} 
professional diversity of backgrounds and a relatively high degree of homogeneity in terms of their focus and expertise.

\section{2. $\quad$ Providing Sufficient Resources}

Enquete Commissions have at their disposal a well-staffed secretariat plus additional staffers for each parliamentary group. Some of the expert members also relied on support for research and drafting. The secretariat considered itself neutral and did not pursue its own agenda.

Its professional attitude garnered trust from Enquete members and proved to be a critical enabler for the work of the Internet Enquete. For a distributed governance group such as the Enquete Commission to work well and to make the best use for subsequent decision-making processes, a highly skilled and committed secretariat is of utmost importance. ${ }^{82}$ Notwithstanding the support by staffers, participation in Enquete Commissions is very demanding. It requires from their members, who typically already have busy schedules, significant commitments over a period of several years.

In order to enable public participation, several members of EIDG successfully advocated the use of the Adhocracy Platform. This collaborative software tool allowed interested observers to submit assessments or recommendations to working groups and, additionally, to rate those drafts by the members of an adhocracy group. The Adhocracy platform constituted an experiment in public participation that various EIDG members regarded with some skepticism. One concern was that introducing a participatory platform would promise a degree of inclusiveness to the public that may end up in disappointment. Indeed, the Adhocracy platform suggests a very low participation threshold, regardless of one's expertise. In fact, the public contributions to the working groups were of mixed quality and the adhocracy platform meant a rise of the workload for the EIDG members who needed to read all of the contributions and, in some cases, communicate with their authors. Nevertheless, the Adhocracy platform has the potential to enhance public participation in a meaningful way if the interface between the actual membership and external participants could be further developed. One promising option is to synthesize public contributions by means of a skilled facilitator who understands the issues and is committed to feed them in a neutral way into the working groups.

\section{3. $\quad$ Compromising Between Degrees of Public Scrutiny}

Although the Internet Enquete explicitly aimed to involve the public in its work, the results did not meet general expectations. The level of participation was lower than expected and integrating public contributions into the process of report writing ended up increasing the already heavy workload of the working groups. To a certain extent, integrating public contributions and running the working groups in an efficient manner constituted conflicting goals. The same can be said about transparency. The streaming of sessions enabled interested observers and the press to closely follow the process and benefit from the expertise brought together. At the same time, the public character of the plenary sessions and some of the working groups meant that the members of the parliament would be more guarded and the chances for mutual learning opportunities diminished. Paradoxically, transparency thus transforms the event that the public deserves to have access to. Finding a compromise between open and closed sessions should be a matter open to experimentation for future Enquete commissions.

\footnotetext{
${ }^{82}$ Some members of the secretariat had academic training.
} 


\section{Translation Into Politics}

Whether and how far the commission's work pays off in the long term has to remain an object of observation. Although the permanent Bundestag's commission Digitale Agenda was institutionalized after elections in 2013, it remains unclear in how far decision-makers will capture results and recommendations of the Commission's work in order to implement policy measures. Up to now, the Enquete process does not provide mechanisms for policy implementation. Nonetheless, finding the appropriate implementation mechanisms that will help inform evidence-based policy making and provide justification for spending further resources should be taken into account in the process design. 


\section{Appendix F: \\ Multistakeholder Approaches to Water \\ Resource Management in the White Volta \\ River Basin}




\title{
NoC Internet Governance Case Studies Series: Multistakeholder Approaches to Water Resource Management in the White Volta River Basin
}

\author{
Rebekah Heacock Jones, Ryan Budish, Sergio Alves, Jr., Sarah Myers West, Rex \\ Troumbley, Sarah Al Saleh, and JeeYoung Oh \\ Berkman Center for Internet \& Society at Harvard University
}

Editorial Note: Context, Character, and Purpose of the Case Study

This case study is part of a globally coordinated, independent academic research pilot project by the Global Network of Interdisciplinary Internet \& Society Research Centers (NoC). Facilitated by the Berkman Center for Internet \& Society at Harvard University, this study examines existing multistakeholder governance groups with the goal of informing the future evolution of the Internet governance ecosystem. Building upon the NETmundial Principles and Roadmap, it contributes to current policy debates at the international level, including the Internet Governance Forum, the NETmundial $\underline{\text { Initiative, }}$ and other organizations and efforts.

Internet governance is an increasingly complex concept that operates at multiple levels and in different dimensions, making it necessary to have a better understanding of both how multistakeholder governance groups operate and how they best achieve their goals. With this need in mind, at a point where the future of Internet governance is being re-envisioned, colleagues from several $\mathrm{NoC}$ institutions around the world have written twelve case studies examining a geographically and topically diverse set of local, national, and international governance models, components, and mechanisms from within and outside of the sphere of Internet governance. Key findings from these cases are summarized in a synthesis paper, which aims to deepen our understanding of the formation, operation, and critical success factors of governance groups and even challenge conventional thinking.

The research, based on twelve case studies, suggests that there is no single best-fit model for multistakeholder governance groups that can be applied in all situations. Rather, it reveals a range of approaches, mechanisms, and tools available for both the formation and operation of such groups. The analysis demonstrates that the success of governance groups depends to a large degree on the careful selection, deployment, and management of suitable instruments from this "toolbox." As governance groups pass through different phases of operation, conveners and facilitators must remain alert to changes in circumstances that necessitate adjustments to the approaches, mechanisms, and tools that they deploy in order to address evolving challenges from inside and outside. This case study provides insights into how those instruments can be deployed and adjusted over time within such groups, and highlights how their interactions with important contextual factors may be successfully managed within given resource restraints.

The research effort is grounded in a diversity of global perspectives and collaborative research techniques. Adhering to objective and independent academic standards, it aspires to be useful, actionable, and timely for policymakers and stakeholders. More broadly, the Network of Centers seeks to contribute to a more generalized vision and longer-term strategy for academia regarding its roles in research, facilitation and convening, and education in and communication about the Internet age.

For additional information on the initiative, please contact Urs Gasser, Berkman Center for Internet \& Society, at ugasser@cyber.law.harvard.edu. 


\begin{abstract}
This case study examines the deployment of the decentralized integrated water resource management (IWRM) model for the management of water resources at both the local and transboundary levels of the White Volta River Basin. The Volta River Basin spans across six countries-Benin, Burkina Faso, Cote d'Ivoire, Ghana, Mali, and Togo. One of the Basin's four main subbasins is the White Volta in Northern Ghana. This case study investigates and compares governance at the sub-basin level through White Volta Basin Board (WVBB) and at the Basin level through the Project for Improving Water Governance in the Volta River Basin (PAGEV). The case study also discusses the development of IWRM in water governance more broadly. The case study concludes that PAGEV was more successful in implementing IWRM principles than the WVBB, but questions remain as to their decision-making processes and the full extent of stakeholder participation.
\end{abstract}




\section{Table of Contents}

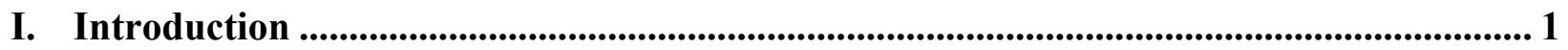

II. Global Trends in Water Governance: Integrated Water Resources Management ......... 1

III. Case Study: the White Volta River Basin in Africa ............................................................... 3

IV. At the National Level: White Volta Basin Board............................................................... 6

A. Values and Functions............................................................................................................6 6

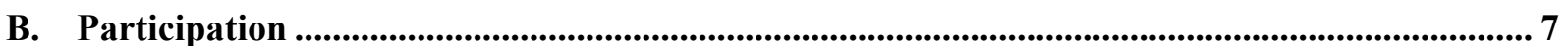

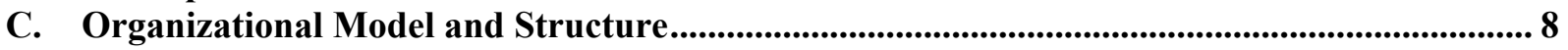

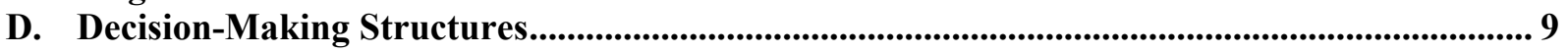

E. Outcome .......................................................................................................................... 9

V. At the Transboundary Level: PAGEV ............................................................................... 10

A. Values and Functions...................................................................................................... 11

B. Participation ............................................................................................................................ 11

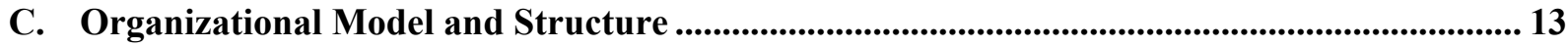

D. Outcome........................................................................................................................ 14

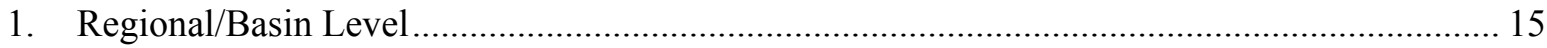

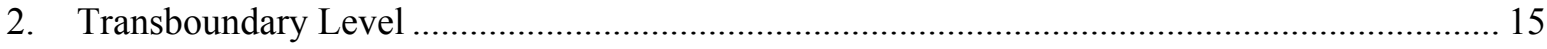

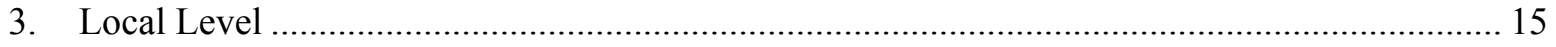

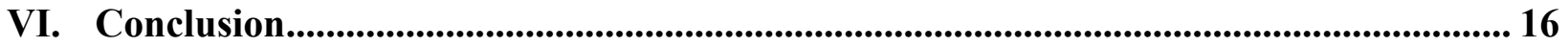




\section{Introduction}

Over the decades, there has been a shift from fragmented approaches to integrated governance in water resource management. First developed in the late 1970s and widely adopted in the beginning of the 1990s, integrated water resources management (IWRM) has quickly become the dominant approach to managing water rights, replacing more sectoral approaches. ${ }^{1}$ By considering the management of shared water resources in a holistic way, IWRM focuses on a participatory approach that involves "users, planners, and policy makers at all levels." 2 The case study of the White Volta River Basin examines the deployment of the decentralized IWRM model for the management of water resources at the local and transboundary levels - the White Volta Basin Board (WVBB) and the Project for Improving Water Governance in the Volta River Basin (PAGEV), respectively_in northern Ghana.

The case study is based on reports, statutes, and studies of IWRM in the White Volta region and in sub-Saharan Africa more generally. Given the remoteness of the White Volta Basin and the relative lack of public documentation on these two groups, accessing detailed information about their current organizational structures, operations, and effectiveness has been difficult. The research has been augmented by interviews with IWRM practitioners with several years of experience deploying the model globally, facilitators present at the inception of the WVBB, and the current WVBB Basin Officer.

The paper is divided into three main parts. First, it examines the development of IWRM in water governance. Then, the paper examines two examples of the application of IWRM to shared water resources in the White Volta River Basin at the local and transboundary levels. The case study concludes that PAGEV was more successful in implementing IWRM principles than the WVBB. Still, questions remain as to how much these institutions embodied multistakeholder governance and how relevant decisions were actually made.

\section{Global Trends in Water Governance: Integrated Water Resources Management}

A global trend in the water sector illustrates a shift away from top-down, supply-driven management of water resources toward bottom-up, demand-driven approaches. Until the midtwentieth century, water management mainly dealt with safety and quantity, such as flood control. From the 1820 s to the 1950 s, sectoral and technical management of water resources was widespread, and each sector involved in water issues in a particular region tended to operate independently. As competition among various water users intensified, however, the lack of coordinated development of available resources created increasing pressure on the management of water resources, especially those that span multiple national boundaries.

\footnotetext{
${ }^{1}$ Colin Mayfield, "Introduction to IWRM," United Nations Water Virtual Learning Centre, http://www.yemenwater.org/wp-content/uploads/2013/03/Introduction-to-IWRM- -History-and-IntroductionCapacity-bui.pdf; see also "Lesson 2: History \& development of IWRM," United Nations Water Virtual Learning Centre.

${ }^{2}$ Jan Hassing, et al., "Integrated Water Resources Management in Action," United Nations World Water Development Reports 3: Water In a Changing World, United Nations World Water Assessment Programme, 2009, http://unesdoc.unesco.org/images/0018/001818/181891E.pdf.
} 
Almost every country in the world has at least one transboundary river - a river that crosses political boundaries - within its borders. Worldwide, approximately 300 rivers run through more than one political boundary, covering over 50 percent of the earth's surface. ${ }^{3}$ Transboundary rivers complicate water governance and management because they often require coordination across different water governance regimes. As a result, departing from the river-alone approach, collaborative river basin management has emerged to minimize harms and provide a holistic approach to managing transboundary rivers.

Since the 1970s, integrated water resource management (IWRM) has emerged as a guiding principle to address the shortcomings of more traditional approaches driven by narrow engineering and sectoral water concerns. IWRM advocates the integration of different sectors and water users and the management of shared waters within the hydrological confines of the river basin. ${ }^{4}$ Traceable back to the 1977 UN Conference on Water, it is a comprehensive approach for achieving sustainable water use that was officially embraced at the 1992 UN Conference on Environment and Development in Rio de Janeiro. ${ }^{5}$ The 2000 Ministerial Declaration of the Hague on Water Security in the 21st Century further emphasized the importance of involving the interests of all stakeholders in the water governance system. ${ }^{6}$

The Global Water Project, ${ }^{7}$ a leading global organization dedicated to advancing sustainable and equitable water governance, defines IWRM as "a process which promotes the coordinated development and management of water, land, and related resources, in order to maximize the resultant economic and social welfare in an equitable manner without compromising the sustainability of vital ecosystems." ${ }^{8}$ While there are disagreements over how it should be put into

\footnotetext{
${ }^{3}$ Andrea K. Gerlak, "Regional Water Governance and Institutional Arrangements Around Transboundary Waters,"48th Annual Meeting of the International Studies Association Chicago, Il. (2007).

${ }^{4}$ Andrea K. Gerlak, Robert G. Varady, and Arn C. Haverland. "Hydrosolidarity and International Water Governance," International Negotiation vol. 14 (2009): p. 320.

${ }^{5}$ A series of international conferences facilitated the policy and scientific evolution of the integrated water resource management (IWRM) and its multistakeholder approach, including the UN Conference on Human Environment (Stockholm, 1972), the International Conference on Water and Environment (Dublin, 1992), the UN Conference on Environment and Development (Rio de Janeiro, 1992), the World Water Forums (various, since 1997), the World Summit on Millennium Development Goals (New York, 2005), and the UN Conference on Sustainable Development (Rio+20, 2012).

6 "Ministerial Declaration of the Hague on Water Security in the 21st Century," March 22, 2000, http://www.worldwatercouncil.org/fileadmin/world_water_council/documents/world_water_forum_2/The_Hague_ Declaration.pdf; K.J. Joy, Suhas Paranjape, and Seema Kulkarni, "Multi-stakeholder Participation, $\bar{C}^{-}$collaborative Policy Making and Water Governance: the Need for a Normative Framework" in Vishwa Ballabh, ed., Governance of Water: Institutional Alternatives and Political Economy (New Delphi: Sage Publications, 2008), p. 270.

${ }^{7}$ The Global Water Partnership (GWP) is a global action network funded in 1996 with support of the World Bank, the United Nations Development Program (UNDP), and the Swedish International Development Cooperation (SIDA), to advance the governance and management of water resources for sustainable and equitable development. In 2002, a GWP Secretariat was established as an intergovernmental organization in Sweden. The network is open to a variety of stakeholders involved in water resources management, such as including government institutions, agencies of the United Nations, development banks, professional associations, research institutions, nongovernmental organizations, and the private sector. The GWP network spans more than 2900 partner organizations in more than 180 countries. It encompasses 13 Regional Water Partnerships, 85 Country Water Partnerships and 2965 Partners in 172 countries. See Global Water Partnership, “About GWP,” http://www.gwp.org/en/About-GWP/. ${ }^{8}$ Global Water Partnership, "What is IWRM?," http://www.gwp.org/en/The-Challenge/What-is-IWRM/.
} 
practice, ${ }^{9}$ the five principles of IWRM developed by the Global Water Partnership are widely accepted:

1. Fresh water is a finite and vulnerable resource, essential to sustain life, development, and the environment.

2. Water development and management should be based on a participatory approach, involving users, planners, and policy makers at all levels.

3. Women play a central part in the provision, management, and safeguarding of water.

4. Water is a public good and has a social and economic value in all its competing uses.

5. Integrated water resources management is based on the equitable and efficient management and sustainable use of water. ${ }^{10}$

IWRM stands out from previous water management efforts because of its integrative approach to shared water resources. It takes into account various aspects of water management, including water uses by sectors, multiple levels of governance, promotion of river basins as management units, and greater participation of stakeholders in water management and decision-making processes. Advocates of IWRM argue that integrated management is necessary to manage shared water resources in an effective and sustainable manner. ${ }^{11}$

While IWRM has come to dominate policy discourse and academic discussion in water resource management as a framework for holistic management of water, it is not without criticism. In a paper outlining the debate surrounding IWRM, Farhad Mukhtarov and Andrea Gerlak write that some opponents of IWRM claim that it does not succeed due to the inherently political processes at play and that some contend that IWRM is too idealistic. ${ }^{12}$ The authors further note that one study of IWRM implementation in Canada shows that historical factors better explain the outcomes of IWRM than institutional design. Thus, some say IWRM has simply become a policy buzzword.

While the debate around IWRM continues, it nevertheless remains a dominant framework in water management around the world, including in the White Volta River Basin in West Africa.

\section{Case Study: the White Volta River Basin in Africa}

The Volta River Basin spans across six countries-Benin, Burkina Faso, Cote d'Ivoire, Ghana, Mali, and Togo - as shown in Figure 1. It is the ninth largest river basin in sub-Saharan Africa. Nearly 20 million people directly or indirectly rely on the resources of the Volta River Basin for their livelihood. ${ }^{13}$ The majority of the basin falls within the territories of Burkina Faso and

\footnotetext{
${ }^{9}$ Farhad Mukhtarov and Andrea K. Gerlak, "Epistemic Forms of Integrated Water Resources Management: Towards Knowledge Versatility,” Policy Sciences vol. 47(2) (2014): p. 101-120.

${ }^{10}$ Global Water Partnership, "IWRM Principles," http://www.gwp.org/en/The-Challenge/What-is-IWRM/IWRMPrinciples/.

${ }^{11}$ Mukhtarov and Gerlak, "Epistemic Forms of Integrated Water Resources Management."

12 Ibid.

${ }^{13}$ Rebecca Welling, Megan Cartin, Désiré Baykono, Ousmane Diallo, International Union for Conservation of Nature (IUCN), "Volta River Basin Ghana \& Burkina Faso: Transboundary water management through multi-level participatory governance and community projects," IUCN Water and Nature Initiative Case Study (2012), p. 2, https://portals.iucn.org/library/efiles/documents/2012-010.pdf.
} 
Ghana: 43 percent and 42 percent, respectively. ${ }^{14}$ The Volta River Basin is divided into the four main sub-basins: the Black Volta, the White Volta, the Lower Volta, and the Oti.

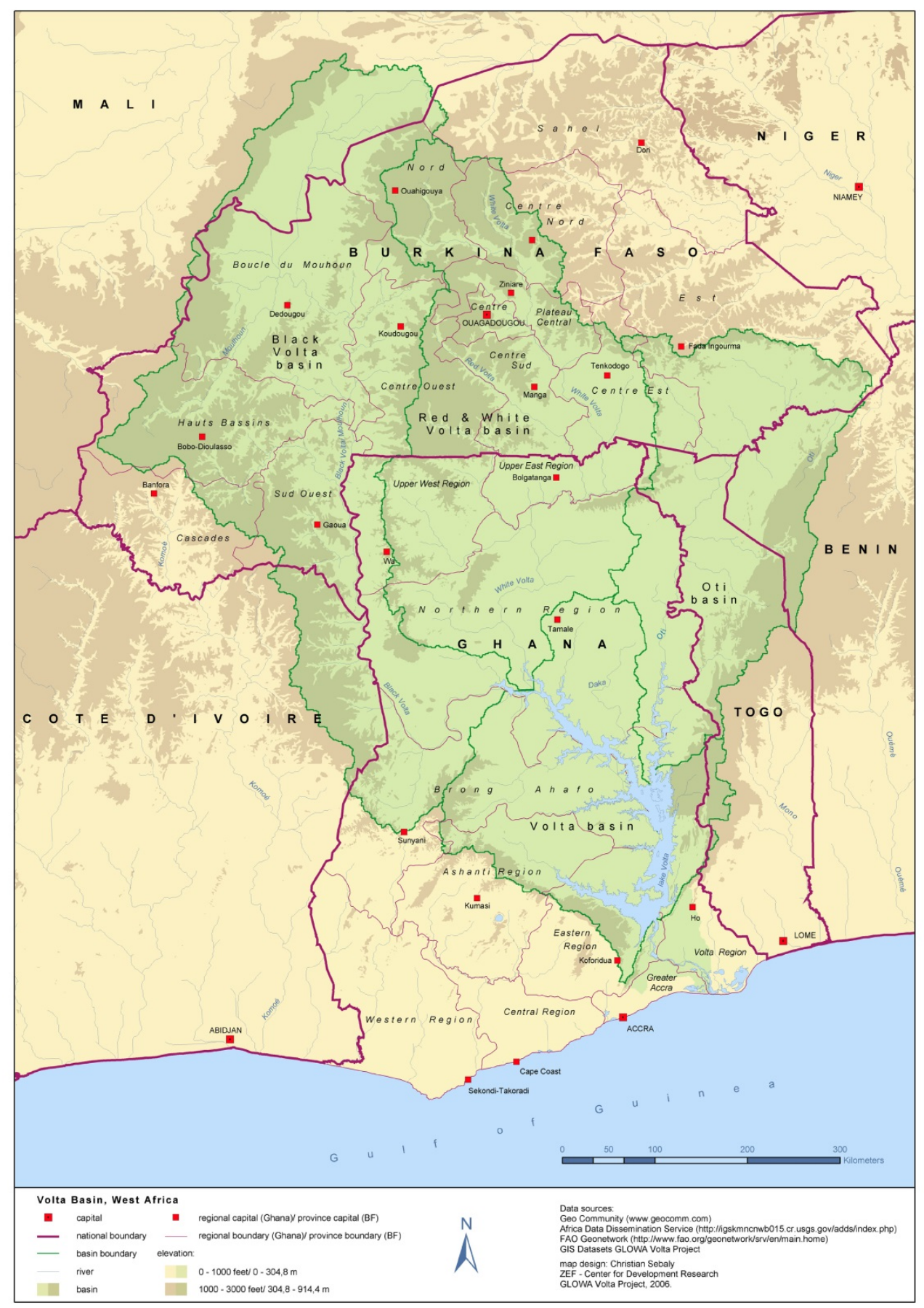

Figure 1. Map of the Volta Basin. GLOWA Volta, The Volta River Basin, available at http://www.zef.de/volta_basin.html.

\footnotetext{
${ }^{14}$ Megan Matthews, "The Volta Convention: An Effective Tool for Transboundary Water Resource Management in an Era of Impending Climate Change and Devastating Natural Disasters?," Denver Journal of International Law \& Policy vol. 41(1) (2012).
} 
The headwaters of the White Volta River begin in Burkina Faso and flow south into Ghana. ${ }^{15}$ A significant portion of the region's population depends on the White Volta for domestic water supply, agriculture, fishing, transportation, and recreation. The river is also critical for electricity/ The Akosombo Dam and Kpong power stations in the lower Volta River system account for approximately 80 percent of the electricity generated in Ghana. In addition to domestic consumption, the Akosombo Dam and Kpong hydropower plants provide electricity to the country's largest aluminum smelter, owned by the Volta Aluminum Company (VALCO), and export surplus power to the neighboring countries, Togo and Benin. ${ }^{16}$

In a historical overview of water management in Burkina Faso and Ghana, Jonathan Lautze, Boubacar Barry, and Eva Youkhana write that after Burkina Faso and Ghana declared independence from their colonial powers (in 1960 and 1957, respectively), the two countries managed their own water-related issues without much consultation or coordination with their neighboring states. Moreover, transboundary disputes were rare within the Volta Basin until the 1990s. No international agreements among riparian states - those situated on the banks of the river-existed that promoted an integrated approach to shared water resources. ${ }^{17}$

Lautze, Barry, and Youkhana state that a lack of integrated water management also persisted among various water-related institutions within riparian states. For example, there was virtually no integration and coordination among the three major government institutions in Ghana-the Volta River Authority, the Ghana Water and Sewerage Corporation, and the Irrigation Development Authority. Each often pursued its own water agenda without much consideration of potential impact on the other institutions' objectives and projects. This siloed approach was perhaps possible due to the relative abundance of water until the 1990s, which allowed policymakers and technocrats to implement water development plans in a fragmented way without immediate serious consequences.

In the 1990s, the Volta Basin experienced severe droughts and floods and faced serious development challenges due to increasing resource demands. For example, in 1998, Ghana suffered from a serious power shortage due to a drought. Ghana accused Burkina Faso of unfairly holding water from the Volta River with its dams. In 1999, Burkina Faso opened its dam spillways, which led to flooding and devastation of downstream Ghana's lands and to a related outbreak of cholera in northern Ghana. ${ }^{18}$ Climate change and the extensive exploitation of water resources also highlighted the need for basin-wide coordination among riparian states. Until this point, Burkina Faso and Ghana rarely coordinated or consulted with each other in their water policy and development initiatives, threatening the sustainability of the management of water resources within the Volta Basin. ${ }^{19}$

Recognizing the merits of the IWRM approach to water management, Ghana underwent a water sector reform process in the 1990s. The Ghanaian Parliament in 1996 approved the Water

\footnotetext{
${ }^{15}$ Matthews, "The Volta Convention," p. 275.

${ }^{16}$ Encyclopedia Britannica, s.v. “Akosombo Dam,” http://www.britannica.com/EBchecked/topic/11743/AkosomboDam; Wisdom Ahiataku-Togobo and Kennedy Amankwa, "Hydro Power Development in Ghana," Presentation (2006), http://www.unido.org/fileadmin/import/52380_Mr._Kennedy_Amankwa.pdf.

${ }^{17}$ Jonathan Lautze, Boubacar Barry, and Eva Youkhana, "Changing Paradigms in Volta Basin Water Management: Customary, national and transboundary," Water Policy vol. 10 (2008): pp. 577-594 at 586.

${ }^{18}$ Matthews, "The Volta Convention," pp. 285-286.

${ }^{19}$ Welling, et al., "Volta River Basin Ghana \& Burkina Faso," p. 3.
} 
Resources Commission Act, which created the Water Resources Commission (WRC). The Act lists several water management functions of the WRC; one is to "propose comprehensive plans for the utilization, conservation, development and improvement of water resources." ${ }^{20}$ The Act requires that the WRC's Board consist of representatives from a range of government agencies, water management organizations, and the state-owned Ghana Water and Sewage Corporation, as well as at least one woman. The Act further enables the WRC to create committees consisting of Board members, non-members, or both to carry out WRC functions and advise the Board.

Similar to Ghana, Burkina Faso carried out IWRM-oriented reforms of its water institutions and policies in the 1990s. In order to secure the World Bank's funding to construct the Ziga dam near Ouagadougou, Burkina Faso had to comply with the World Bank's transboundary waters policy, which promoted coordination among the affected riparian states. The government negotiated a "no objection" agreement with Ghana for its dam construction project, one of the first examples of formal coordination and collaboration between Ghana and Burkina Faso in the management of the Volta River Basin. ${ }^{21}$

As the two countries embraced IWRM in their water policy, the White Volta Basin Board was created at the national level in Ghana and PAGEV at the transboundary level between Ghana and Burkina Faso. The following sections examine the implementation of IWRM models at these two levels.

\section{At the National Level: White Volta Basin Board}

In 2004, the WRC established the first of Ghana's Basin Boards, which are tasked with implementing the functions of the WRC at the local level and bringing together multiple stakeholders. The first Board was established in the Densu River Basin, which provides water to Ghana's capital, Accra. Two years later, the WRC approved a draft operational guideline for the White Volta Basin Board (WVBB), ${ }^{22}$ which was officially established in July $2006 .{ }^{23}$ Three additional Basin Boards - the Ankobra Basin Board, the Pra Basin Board, and the Tano Basin Board-have since been established. ${ }^{24}$ The WVBB was selected for the case study because it allows for parallel comparison to the transboundary-level water governance between Burkina Faso and Ghana in the second half of the paper.

\section{A. Values and Functions}

The WVBB's stated vision is to achieve "sustainable water management by all for all." Its operational guidelines further define its mission as:

\footnotetext{
${ }^{20}$ Water Resources Commission Act, Act 552 of 1996 (Republic of Ghana), available at http://faolex.fao.org/docs/pdf/gha10691.pdf. Note: the Act does not specifically mention IWRM; interestingly, the Ghana Water Resources Commission's website misquotes this first listed function as “propos[ing] integrated water resources management plans to guide the utilization, conservation, development and improvement of water resources.” Water Resources Commission of Ghana, “About Us,” http://wrc-gh.org/en/about-us.

${ }^{21}$ Lautze, Barry, and Youkhana, "Changing Paradigms in Volta Basin Water Management," p. 589.

${ }^{22}$ Water Resources Commission, “Operational Guidelines of the White Volta Basin Board,” December 2006, archived at https://perma.cc/H83T-3M2V?type=pdf.

${ }^{23}$ Water Resources Commission of Ghana, "Basins-White Volta," http://wrc-gh.org/en/basins/19/white-volta; see also GLOWA, "GLOWA Volta-Overview," http://www.glowa.org/eng/volta_eng/volta_eng.php.

${ }^{24}$ Water Resources Commission of Ghana, "Basins," http://wrc-gh.org/en/basins.
} 
To regulate and manage the sustainable utilization of water resources and to coordinate related policies by combining our core competencies and hard work through effective participation, monitoring and awareness creation for socio-economic development of the White Volta Basin and Ghana as a whole. ${ }^{25}$

Among the WVBB's stated functions are managing water resource development in the river basin, monitoring and evaluating programs for water resource utilization and management, collaborating with related organizations, requesting that the WRC formally engage with consultants and advisors as it deems necessary, and providing advice to the WRC on issues affecting water resources in the basin. The Board's operational guidelines state that its mission and vision must align with those of the WRC.

\section{B. Participation}

In September 2005, the WRC, the GLOWA Volta Project, ${ }^{26}$ and the International Food Policy Research Institute (IFPRI) held a joint stakeholder workshop to select the initial members of the WVBB. ${ }^{27}$ The operational guidelines define WVBB membership as consisting of a Chairperson (the Basin Officer) appointed by the WRC and drawn from the stakeholder group listed below, a representative of the WRC, and one person from each of the following stakeholder groups. ${ }^{28}$

- Metropolitan/Municipal/District Assemblies (8 representatives) ${ }^{29}$

○ Bawku Municipal Assembly

- Bawku West District Assembly

- Bolgatanga Municipal Assembly

- Bongo District Assembly

- Garu-Tempani District Assembly

- Kassena-Nankana District Assembly

- Talensi-Nabdam District Assembly

- West Mamprusi District Assembly

- Regional Administration (1 representative)

- Regional planning and coordinating unit of the Upper East Regional coordinating council, which itself includes representatives from the 13 districts that make up the region. ${ }^{30}$

- Water Users (1 representative)

○ Ministry of Food and Agriculture

- Regulatory Institutions (1 representative)

$\circ$ Environmental Protection Agency

\footnotetext{
${ }^{25}$ Water Resources Commission, "Operational Guidelines of the White Volta Basin Board."

${ }^{26}$ The GLOWA Volta project is led by the German Centre for Development Research on global climate change and hydrological cycle.

${ }^{27}$ Joseph Boateng Agyenim, "Investigating Institutional Arrangements for Integrated Water Resource Management in Developing Countries: The Case of White Volta Basin, Ghana" (Ph.D. dissertation, VU University Amsterdam, 2011), p. 163.

${ }^{28}$ Water Resources Commission, “Operational Guidelines of the White Volta Basin Board,” pp.2-3.

${ }^{29}$ Neither the Water Resources Commission of Ghana website nor the White Volta Basin Board operational guidelines have been updated to reflect the creation of multiple new districts in the Upper East region of Ghana since 2006. It is possible that the WVBB now includes additional representatives from new districts.

${ }^{30}$ Ghana Districts, "Upper East," http://www.ghanadistricts.com/region/?r=8.
} 
- Research Institutions (1 representative)

- Savannah Agricultural Research Institute (SARI)

- Non-Governmental Organizations (1 representative)

- NGO Forum on Water and Sanitation

- Traditional Authorities (1 representative)

○ Upper East Regional House of Chiefs

- Women's Representative (1 representative)

- Department of Women (Ministry of Women's and Children's Affairs)

- Water Providers (1 representative)

$\circ$ Community Water and Sanitation Agency

WVBB members serve three-year terms with no term limits. Members of the WVBB can be removed by the WRC for failure to perform their required duties or for misconduct. ${ }^{31} \mathrm{New}$ members are appointed by the organizations they represent or, in the case of the Chairperson, by the WRC. With the exception of the representatives from the Savannah Agricultural Resource Institute (which hosts the GLOWA Volta Project) and the NGO Forum on Water and Sanitation, the WVBB membership is dominated by elected representatives and governmental bodies. ${ }^{32}$ The fact that government participants were so central to the membership of the WVBB raises questions about how effectively non-governmental participants could participate. ${ }^{33}$

\section{Organizational Model and Structure}

The WVBB is organized around the four sub-committees: ${ }^{34}$

- The sub-committee on Programs and Budget prepares and facilitates the Board's budget, as well as sources and implements funds for programs of the Board. ${ }^{35}$

- The subcommittee on Land Use, Research, Education and Public Awareness identifies research needs and plans and organizes public awareness programs on topics critical to the Basin. ${ }^{36}$

- The subcommittee on Environment, Utilization, and Regulation registers water users while ensuring compliance with water use and environmental regulations. ${ }^{37}$

\footnotetext{
${ }^{31}$ Water Resources Commission, "Operational Guidelines of the White Volta Basin Board."

${ }^{32}$ Agyenim, "Investigating Institutional Arrangements," p. 163. The GLOWA Volta Project provides analysis of environmental change's effects on water resources in the region from an integrative and data-driven interdisciplinary approach. And, the IFPRI acts as an informal external advisor, conducting research on socio-economic aspects of water resource management within the White Volta Basin and evaluating the performance of dams for irrigation purposes in the basin.

${ }^{33}$ Schiffer et al., "Who has influence in Multistakeholder Governance Systems?," p. 12.

${ }^{34}$ Ibid., p. 163.

${ }^{35}$ Agyenim, "Investigating Institutional Arrangements," p. 145. The sub-committee's five members are the WVBB representatives from the Bolgatanga Municipal Assembly, Ministry of Food and Agriculture, Regional Coordinating Council, Regional House of Chiefs, and Kassena-Nankana District Assembly.

${ }^{36}$ Ibid., p. 146. This sub-committee has five members: one Board member each from the Savanna Agricultural Research Institute (SARI), Bawku West District Assembly, NGO Forum on Water and Sanitation, Bongo District Assembly, and WRC.

${ }^{37}$ Representatives from the Community Water and Sanitation Agency, Environmental Protection Agency, Ministry of Food and Agriculture, West-Mamprusi District, and Talensi-Nabdam District Assemblies serve on this committee.
} 
- The subcommittee on Transboundary Issues maintains a strong collaboration between the WVBB, the Ghana-Burkina Faso transboundary structures, and the Nakambe Water Agency of Burkina Faso. ${ }^{38}$

- The Basin Officer, appointed by the WRC and the only full-time employee of the WVBB, coordinates activities, develops and implements action plans for Board projects, and reviews progress towards objectives. ${ }^{39}$

The WVBB operational guidelines describe the WVBB as collaborating with other organizations and require the secretary to publish reports to the WRC, but do not require the WVBB to publicly publish meeting minutes or otherwise include members not named as formal participants.

\section{Decision-Making Structures}

The WVBB's operational guidelines state that issues before the Board are to be decided by a simple majority vote, with each member voting and with 50 percent plus one of the members constituting a quorum. The guidelines further stipulate that final decision-making authority rests with the WRC. Given that WVBB decisions are subject to approval or revision by the WRC, the decision-making structure appears to be aligned more closely with a hierarchical model, rather than a truly distributed approach. ${ }^{40}$

A 2010 discussion paper from the IFPRI examines who has influence in the WVBB in practical terms (as opposed to who is represented formally on the board). ${ }^{41}$ The paper illustrates that the formal decision-making structure does not necessarily dictate the decision-making dynamics of the Board. The study uses a participatory network mapping method to analyze how social networking influences decision making in the WVBB. The study finds that active social networking in information exchange contributes more to the outcome than the formal lines of command and funding. If stakeholders participate more prominently in exchanging information and providing more advice to others, they are perceived to be more influential and can offset the overrepresentation of government actors on the Board. Although some government organizations are central in giving funding and command, they exhibit a low level of influence. The paper concludes that a space allowing the exchange of information and advice among stakeholders is critical in distributed governance.

\section{E. Outcome}

According to the WRC's website, the WVBB has conducted a number of "interventions" in the White Volta River Basin, including:

\footnotetext{
${ }^{38}$ Agyenim, "Investigating Institutional Arrangements," p. 146. The sub-committee consists of representatives from Bawku Municipal Assembly, Department of Women, Garu-Tempani District Assembly, Regional House of Chiefs, and the Regional Coordinating Council. Nakambe is the name of the White Volta River in Burkina Faso.

${ }^{39}$ Eric Antwi Ofosu, "Sustainable Irrigation Development in the White Volta Sub-Basin," (Ph.D. Dissertation, UNESCO-IHE Institute for Water Education, 2011).

${ }^{40} \mathrm{Ibid}$., p. 161. As Joseph Boateng Agyenim has noted in his study of IWRM in the White Volta region, how this form of "joint decision-making" is interpreted depends upon how authority is distributed. He summarizes, "if authority is equally shared, the stakeholders negotiate a consensus or practice voting according to defined majority rules. In hierarchical relationships . . . joint decision-making involves lower-level authorities making decisions that are then approved or changed by higher-level authorities." Agyenim, "Investigating Institutional Arrangements," p. 157.

${ }^{41}$ Schiffer et al., "Who has influence in Multistakeholder Governance Systems?."
} 
- facilitating the reduction in farming activities along the river banks through rehabilitation of a broken dam to create an alternative water source for irrigation;

- holding sensitization, capacity building, and planning meetings with technical service providers (forestry, agriculture, and planners) and communities;

- holding transboundary joint community fora on river bank protection interventions between Ghana and Burkina Faso;

- establishing a local trans-boundary committee on the management of the White Volta River Basin and adoption of code of conduct and operational guidelines for this committee; and

- water quality monitoring of selected locations in Burkina Faso and Ghana. ${ }^{42}$

However, the WRC does not describe the WVBB's level of involvement in the aforementioned achievements. And, it is unclear to what extent these interventions were jointly developed or approved by the WVBB and whether external stakeholders were consulted. It is difficult to determine who has been making substantive decisions and how much of the decision-making process involves the WVBB.

Furthermore, there is some evidence to suggest that the WVBB has been largely inactive or ineffective in implementing IWRM. The WVBB does not seem to regularly convene, let alone meet once every three months as required by the operational guidelines. ${ }^{43}$ According to one record, the WVBB met only once between 2011 and 2012. ${ }^{44}$ Additionally, a dissertation published in 2011 on irrigation in the White Volta region found:

Even though the White Volta sub-basin Management Board has been established for a few years, it has made little impact on the Water Resources Management in the subbasin. For example the National Water Policy which demands registration of water uses and granting of permit from some specific water uses has not been carried out in the basin. ${ }^{45}$

The dissertation later asserts that "it can be concluded that the Volta basin has a complex formal structure that largely exists on paper only, ${ }^{46}$ a conclusion shared by Eva Schiffer, a researcher who conducted training workshops for the WVBB in 2005 and $2006 .{ }^{47}$ Other accounts of the WVBB note that it is underfunded, understaffed, and that stakeholders are often not equipped to effectively monitor water issues in the region. ${ }^{48}$

\section{At the Transboundary Level: PAGEV}

In April 2004, Ghana and Burkina Faso signed the "Ghana-Burkina Joint Declaration." The declaration acknowledged both countries' common environmental and water issues and

${ }^{42}$ Water Resources Commission, "Basins—White Volta," http://wrc-gh.org/en/basins/19/white-volta.

${ }^{43}$ Water Resources Commission, "Operational Guidelines of the White Volta Basin Board," p. 5.

${ }^{44}$ Water Resources Commission, “Annual Report 2012,” 2012, p. 38, http://doc.wrc-

gh.org/pdf/WRC\%20Annual\%20Report\%202012_Final\%201.pdf.

${ }^{45}$ Ofosu, "Sustainable Irrigation Development," p.31-32.

${ }^{46}$ Ibid., p. 40.

${ }^{47}$ Eva Schiffer, "Research Project on the White Volta Basin Board," July 4, 2014.

${ }^{48}$ Ibid.; Ofosu, "Sustainable Irrigation Development," p. 32; Karen Greenough et al., "Stories of Change and Innovation in the Volta Basin Development Challenge Program," CIGAR Challenge Program for Water and FoodVolta Basin (March 2014), https://cgspace.cgiar.org/handle/10568/35126. 
expressed a desire to collaborate on integrated management of the shared water. ${ }^{49}$ The Project for Improving Water Governance in the Volta Basin (Projet d'Amelioration de la Gouvernance de l'Eau dans le basin de la Volta, or PAGEV) is an effort to support collaborative water resource management between Burkina Faso and Ghana. As part of the project, both countries agreed to pilot IWRM interventions in transboundary river basins. The International Union for Conservation of Nature (IUCN) and Global Water Partnership launched the PAGEV project in July 2004 through its Water and Nature Initiative (WANI) ${ }^{50}$ and with support from the Government of the Netherlands and the Swedish International Development Agency. ${ }^{51}$ Nongovernmental organizations and international donors played a significant role in creating a multilevel partnership to promote transboundary water management and sustainable development.

\section{A. Values and Functions}

PAGEV aims to improve water governance in the Volta River Basin by promoting public participation and consensus on key water management principles and working to develop institutional capacity for coordination mechanisms. ${ }^{52}$

According to its 2007 annual report, PAGEV focuses on four strategic areas:

- Key decision-support information base: inform constructive dialogues and collaboration on water management between Ghana and Burkina Faso.

- Pilot IWRM interventions: jointly develop, implement, and learn from pilot IWRM interventions in select transboundary sub-basins.

- $\quad$ Policy and institutional change: broaden and strengthen existing bilateral consultations mechanisms through the adoption and implementation of a code of conduct in the management of shared waters.

- Learning and coordination: manage and coordinate project, support structured learning, and make available lessons learned. ${ }^{53}$

\section{B. Participation}

The PAGEV Steering Committee convenes a meeting two or three times a year, and the codirectors of the project-Ghana's WRC and Burkina Faso's Directorate General of Water Resources (DGRE) - jointly chair the meeting. Based on the 6th and 7th Steering Committee meeting minutes, the following participants are identified as the Steering Committee members:

- PAGEV Steering Committee

- Executive Secretary of WRC, Ghana

- Director General, DGRE, Burkina Faso

- Head of Programme/Executive Secretary, GWP/WAWP, Burkina Faso

- Director General of Rural Property and Farming Operations/MAHR, Burkina Faso

\footnotetext{
${ }^{49}$ Matthews, "The Volta Convention," p. 293.

${ }^{50} \mathrm{WANI}$ is a worldwide initiative of the IUCN that seeks to demonstrate how to mainstream the ecosystem approach into river basin planning and management.

${ }^{51}$ IUCN, "Volta River Basin,"

http://www.iucn.org/about/work/programmes/water/wp_where_we_work/wp_our_work_projects/wp_our_work_vrb /.

${ }^{52}$ Welling, et al., "Volta River Basin Ghana \& Burkina Faso."

${ }^{53}$ Project for Improving Water Governance in the Volta Basin (PAGEV), "2007 Progress Report: 1st January-30th September," (2007), http://cmsdata.iucn.org/downloads/pagev_report_jan_sept2007_ver_apr08.pdf.
} 
- Representative of the Ministry of Foreign Affairs and Regional Cooperation, Burkina Faso

- Regional Coordinator of Programmes, IUCN-BRAO, Burkina Faso or Coordinator of Delta Programme, IUCN-BRAO, Mali

- PAGEV Coordinator and Secretary to the Committee, Burkina Faso ${ }^{54}$

While there are a few representatives from non-profit organizations, the Steering Committee is largely composed of government officials. Little information is available on how the Steering Committee members are selected. Other participants in Steering Committee meetings include observers from government agencies, local governments, donors, and NGOs.

In order to assemble a viable network as an instrument for promoting cooperation, PAGEV has sought partnerships with communities, local and national governments, and international organizations. For example, it has signed memoranda of understanding with two communitybased NGOs from Burkina Faso and Ghana: BISSAKOUPOU and ZOVFA. The MOUs establish partnerships to support participatory processes and to empower stakeholders for water governance and conflict resolution in the pilot sub-basin. The 2005 annual report identifies the following partners: ${ }^{55}$

- Community Level Stakeholders

- Sampema, Zekeze, Beleyerla, and Mogr-nore Villages: 4 communities in the target subbasin for pilot interventions in Burkina Faso; 3 representatives from each village

- Mognore, Sapeliga, Kugrasia and Sakom Villages: 4 communities in the target sub-basin for pilot interventions in Ghana; 3 representatives from each village

- BISSACOUPOU (community-based NGO in Burkina Faso)

- ZOVFA (community-based NGO in Ghana)

- Decentralized Local Government Agencies

- Departments of Bittou and Zabre in Burkina Faso

- Bawku Municipal, Bawku-West, and Garu-Tempane District Assemblies in Ghana

- Technical directorates of Ministries of Agriculture, Environment, Forestry, and Livestock in both countries

- National and Regional Government Agencies

- Ministry of Agriculture, Fisheries, and Water, Burkina Faso

- Ministry of Water Resources, Works, and Housing, Ghana

- Ministries of Regional Cooperation of Burkina Faso and Ghana

- Direction Generale de l'Inventaire et des Ressources Hydrauliques (DGIRH) of Burkina Faso

- Water Resources Commission of Ghana

- Sub-regional/International Organizations

- IUCN West Africa Regional Office (UICN-BRAO)

- GWP/West Africa Water Partnership

- Donors

- Sida of Sweden

- DGIS of the Netherlands

\footnotetext{
${ }^{54} \mathrm{Ibid}$.

${ }^{55}$ PAGEV, "Progress Report 2005,” (2005).
} 


\section{Organizational Model and Structure}

PAGEV promotes the IWRM framework of a multi-level, participatory decision-making process, involving grassroots committees and villages, national-level committees, the Local Transboundary Committee, and the Volta Basin Authority. These cross-sectoral connections facilitate communication among all stakeholders and help to build trust and capacity across the basin. ${ }^{56}$ Key participants include:

- Local level: Eight grassroots communities (four in Ghana and four in Burkina Faso) were selected to implement the pilot activities, including a joint project to restore riverbanks on both sides of the border.

- National level: PAGEV facilitates national forum meetings to consolidate local partnerships. The forums develop plans that harmonize local initiatives, taking into account local and national priorities. Furthermore, they enable the input of local communities into decision-making processes at the regional level.

- Transboundary level: The Local Transboundary Committee has 14 members, seven representatives each from Ghana and Burkina Faso. The committee coordinates joint activities across the border, addresses local-level water use problems and disputes, and strengthens cooperation between the two countries.

- Regional/basin level: The ministers from the six Volta basin states-Ghana, Burkina Faso, Benin, Togo, Mali, and Cote d'Ivoire - created the Volta Basin Authority in 2007 to manage water and other related resources in the Volta Basin in a holistic manner.

The WVBB is part of PAGEV's multi-level, participatory ecosystem of water resources management in Ghana and Burkina Faso. It interacts with local communities, national fora, and the Local Transboundary Committees at three different levels, as illustrated in Figure 2.

${ }^{56}$ Welling, et al., "Volta River Basin Ghana \& Burkina Faso," p. 4-5. 


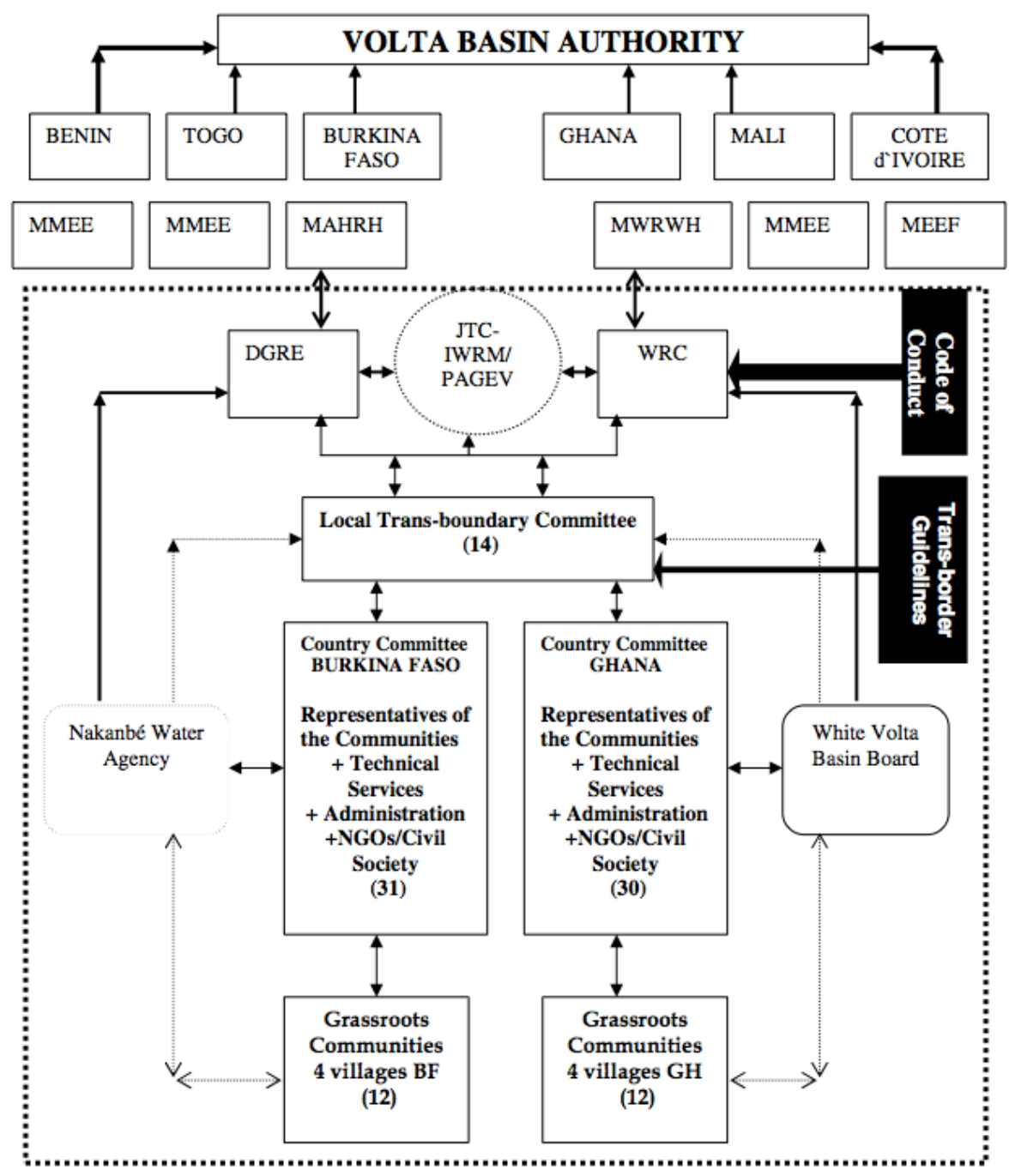

Figure 2. Institutional framework for the management of the Volta Basin. The World Conservation Union (IUCN), PAGEV Project Completion Report, July 2004 to September 2007 (Oct. 2007), http://cmsdata.iucn.org/downloads/pagev_completionreport_2004_2007_eng.pdf.

\section{Outcome}

The first phase of the PAGEV project lasted from 2004 to 2007. While there is limited information on its activities after 2007, a 2011 IUCN report writes that the second phase (most likely from 2008 to 2011) of PAGEV focused on supporting the endorsement of the Volta Basin Authority strategic plan and the establishment of a basin-wide information system. ${ }^{57}$ Another IUCN report that is written in 2012 or after mentions planning for a third phase of PAGEV to continue to support the implementation of the Volta Basin Authority's plan. ${ }^{58}$ The fact that donors continue to fund PAGEV in extended phases suggests that it continues to make progress in improving water usage in the Volta River Basin.

\footnotetext{
${ }^{57}$ IUCN, "Water Vision to Action: Catalyzing Change through the IUCN Water \& Nature Initiative- Results Report," (2011), http://cmsdata.iucn.org/downloads/final_wani_results_report_lr.pdf.

${ }^{58}$ Welling, et al., "Volta River Basin Ghana \& Burkina Faso," p. 14.
} 


\section{Regional/Basin Level}

Assisted by PAGEV, the six basin countries signed a Convention on the Status of the Volta River in 2007, which established the Volta Basin Authority. By 2010, five of the six countries had ratified the agreement, including Mali, Ghana, Togo, Benin, and Burkina Faso, bringing the Convention into effect. The Convention's mandate promotes "the implementation of integrated water resources management and the equitable distribution of the benefits resulting from their various utilizations.. ${ }^{59}$ According to a report from the IUCN, the Volta Basin Authority has actively facilitated workshops and forums at the regional level, providing platforms for dialogue and discussion for stakeholders to reconcile national problems through regional approaches. The Volta Basin Authority's statutes recognize a "Forum of the Parties involved in the Volta Basin Development" as a permanent administrative structure. The forum includes representatives from:

- Various categories of water users of the member states;

- Civil society involved in the management of water resources

- Decentralized local authorities in each portion of the basin of the state parties

- Representatives of the National Focal Structures;

- Representatives of the neighboring transboundary basin organizations; and

- Representatives of research centers in the water and environment sector. ${ }^{60}$

The IUCN report further states that the IUCN has scaled up PAGEV experiences to other riparian states in the Volta Basin by reinforcing capacities of experts from all the riparian countries and supporting the development of the Volta Basin Authority's plans.

\section{Transboundary Level}

Ghana and Burkina Faso agreed to develop a Code of Conduct for the Sustainable and Equitable Management of the Water Resources of the Volta Basin to enable various stakeholders to come together and create a common goal around accepted knowledge and information. In 2006, national validation workshops were convened in each country to ensure input was received from government agencies, NGOs, and civil society groups. With support from WANI, the Joint Technical Committee on IWRM (JTC-IWRM) hosted a harmonization workshop with representation from both countries that led to finalization of the Code in June 2006.

The Code of Conduct has eight sections and 59 articles, and outlines a framework for decision making and action in the management of shared water resources between Ghana and Burkina Faso. The Code emphasizes the management principles of the Volta River Basin's natural resources related to sustainable development, integrated water resources management, cooperation, and governance.

\section{Local Level}

In 2009, PAGEV supported regional workshops and forums to discuss water for agriculture and sustainable underground resource management. PAGEV also supported a number of local development activities, including community efforts around riverbank protection. PAGEV Coordinator Kwame Odame-Ababio reports that the local committees planted trees along nearly

\footnotetext{
${ }^{59}$ Matthews, "The Volta Convention," p. 294.

${ }^{60}$ Ben Y. Ampomah, Bernadette A. Adjei, and Eva Youkhana, "The Transboundary Water Resources Management Regime of the Volta Basin," ZEF Working Paper Series 28, Department of Political and Cultural Change, University of Bonn (2008).
} 
16 kilometers of riverbank to reduce soil erosion and protect water quality. ${ }^{61}$ The 2006 annual report notes that an exchange of visits among the communities was organized to facilitate the sharing of information and experiences. ${ }^{62}$

As was the case with the WVBB, it is difficult to determine to what extent PAGEV directly played a role in the aforementioned achievements. It is also unclear how frequently partners were consulted in the decision-making process. Although the decision-making structure of PAGEV is not explicitly known, it held regular steering committee meetings and produced annual reports chronicling its activities and progress, which suggests that it has been more active than the WVBB.

\section{Conclusion}

The lack of specific information about the activities of the White Volta Basin Board makes it difficult to ascertain whether the governance model the Board embodies is functional, and to what extent the Board has successfully implemented the multistakeholder principles of IWRM. Studies and interviews indicated that the Board faces significant resource challenges that may prevent it from fully carrying out its mission.

On a broader scale, however, IWRM in northern Ghana appears to have met with some success. PAGEV has actively engaged local, regional, and national stakeholders in a number of workshops, dialogues, and development efforts related to water resource management, strengthening participation and cooperation in the region. PAGEV assisted in the creation of several groups and institutions (e.g., the local communities, Local Transboundary Committee, and the Volta Basin Authority) that embody IWRM principles. PAGEV was perhaps more successful than the WVBB because greater financial investment and human resources were available from international organizations.

The case study offers several lessons. First, PAGEV's 2012 progress update indicates that it has had the most success with participation when the "tangible results and improvements in their everyday lives" are readily apparent to stakeholders. Second, as the IFPRI study shows, a space that allows stakeholders to exchange information and provide advice can counterbalance formal decision making and funding structures in which governments play a central role. Third, the multi-level, participatory governance model can better synchronize policy across various stakeholders, especially when coordinating with other states.

Two questions remain open. First, the case study cannot conclusively determine the extent to which the decision-making processes of the WVBB and PAGEV fully incorporated the views of all stakeholders. Participation by civil society, businesses, and researchers was limited in the WVBB; and, while PAGEV has actively facilitated multistakeholder forums and IWRM governance at multiple levels, there is little information on how its steering committee made decisions and to what extent partners were involved in substantive decision making. Because of the lack of clarity regarding decision-making processes, there is also an open question about the

\footnotetext{
${ }^{61}$ Kwame Odame-Ababio, "Stimulating Stakeholders' Support for Managing Shared Waters-Experiences From the Volta Basin," International Symposium on Transboundary Waters Management, Thessalonica, Greece (October 2008).

${ }^{62}$ IUCN, “Annual Report-2006-Regional Office for West Africa,” (2006), https://portals.iucn.org/library/efiles/documents/2006-078.pdf.
} 
role that various parties played in agenda setting within the organizations. Particularly, it would be important to know about the extent to which the various stakeholders (including civil society, funders, and citizens more broadly) were able to shape the direction and agenda of the organizations of as a whole. 


\section{Appendix G: \\ Multistakeholder Governance and Nodal Authority - Understanding Internet Exchange Points}




\title{
NoC Internet Governance Case Studies Series: Multistakeholder Governance and Nodal Authority - Understanding Internet Exchange Points ${ }^{1}$
}

\author{
Ben Wagner and Patricia Mindus \\ European University Institute and the Philosophy Department of Uppsala University
}

Editorial Note: Context, Character, and Purpose of the Case Study

This case study is part of a globally coordinated, independent academic research pilot project by the Global Network of Interdisciplinary Internet \& Society Research Centers (NoC). Facilitated by the Berkman Center for Internet \& Society at Harvard University, this study examines existing multistakeholder governance groups with the goal of informing the future evolution of the Internet governance ecosystem. Building upon the NETmundial Principles and Roadmap, it contributes to current policy debates at the international level, including the Internet Governance Forum, the NETmundial Initiative, and other organizations and efforts.

Internet governance is an increasingly complex concept that operates at multiple levels and in different dimensions, making it necessary to have a better understanding of both how multistakeholder governance groups operate and how they best achieve their goals. With this need in mind, at a point where the future of Internet governance is being re-envisioned, colleagues from several NoC institutions around the world have written twelve case studies examining a geographically and topically diverse set of local, national, and international governance models, components, and mechanisms from within and outside of the sphere of Internet governance. Key findings from these cases are summarized in a synthesis paper, which aims to deepen our understanding of the formation, operation, and critical success factors of governance groups and even challenge conventional thinking.

The research, based on twelve case studies, suggests that there is no single best-fit model for multistakeholder governance groups that can be applied in all situations. Rather, it reveals a range of approaches, mechanisms, and tools available for both the formation and operation of such groups. The analysis demonstrates that the success of governance groups depends to a large degree on the careful selection, deployment, and management of suitable instruments from this "toolbox." As governance groups pass through different phases of operation, conveners and facilitators must remain alert to changes in circumstances that necessitate adjustments to the approaches, mechanisms, and tools that they deploy in order to address evolving challenges from inside and outside. This case study provides insights into how those instruments can be deployed and adjusted over time within such groups, and highlights how their interactions with important contextual factors may be successfully managed within given resource restraints.

The research effort is grounded in a diversity of global perspectives and collaborative research techniques. Adhering to objective and independent academic standards, it aspires to be useful, actionable, and timely for policymakers and stakeholders. More broadly, the Network of Centers seeks to contribute to a more generalized vision and longer-term strategy for academia regarding its roles in research, facilitation and convening, and education in and communication about the Internet age.

For additional information on the initiative, please contact Urs Gasser, Berkman Center for Internet \& Society, at ugasser@cyber.law.harvard.edu.

\footnotetext{
${ }^{1}$ The authors can be reached at bwagner@europa-uni.de or https://cihr.eu/contact/ and patricia.mindus@filosofi.uu.se. The paper was developed as part of a research project on Internet Governance at European University Institute with Giovanni Sartor and Alex Trechsel.
} 


\begin{abstract}
This case study considers Internet exchange points (IXPs) as an example of governance processes in action. Internet exchange points are the points of connection between different Internet networks, which enable different networks to exchange traffic at a shared facility without cost to either party through a process known as "peering". Three different IXP governance models representing large and influential IXPs are compared: the DE-CIX in Frankfurt, CAIX in Cairo, and KIXP in Nairobi. DE-CIX, the largest IXP in the world, is a subsidiary of the German Internet trade association eco, and is thus "owned" by the Internet industry in Germany. Though well functioning, this has meant that key stakeholder groups such as civil society, and the academic and technical communities are excluded from participating in discussions over policy decisions. In contrast, the Cairo Internet Exchange Point (CAIX) is run by a public authority, the Egyptian Ministry of Communications and Information Technology. Though it is governed by a broad set of stakeholders including private sector, government, and civil society representatives, its decision-making processes are somewhat opaque. Lastly, KIXP was founded by a Kenyan network engineer and is governed by a local trade association. While set up with multistakeholder coordination under the leadership of the private sector, its dayto-day operations and governance fall under private sector control. By tracing out the plurality of models used for IXP governance and comparing the processes of developing peering relationships, this case provides unique lessons for the governance process, particularly surrounding trade-offs between inclusiveness and effectiveness.
\end{abstract}




\section{Table of Contents}

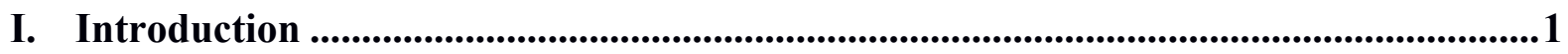

II. A Brief Overview: What Are IXPs and Where Do They Come From?........................1

III. Studying IXP Governance ..................................................................................................4

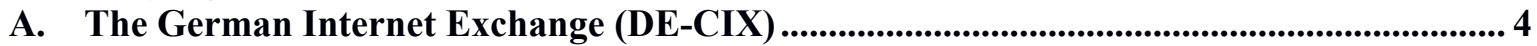

B. The Cairo Internet Exchange Point (CAIX) in Egypt..................................................6 6

C. Kenya Internet Exchange Point (KIXP) ..................................................................... 7

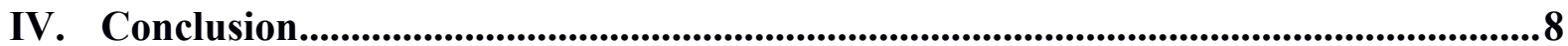




\section{Introduction}

Multistakeholder governance has spread throughout Internet governance in many shapes and forms. One of the most interesting examples is the governance of Internet Exchange Points (IXPs), the points of connection between different Internet networks that are crucial to the functioning of the Internet as a whole (Chatzis, Smaragdakis, \& Feldmann, 2013). In particular, they enable different networks to exchange traffic at a shared facility without cost to either party, a process known as "peering." IXPs are also important because they have produced such a diverse number of rules and procedures by which they are governed, warranting far greater academic inquiry than has been the case (Ryan \& Gerson, 2012). The governance structures range from private for-profit, private non-profit, quasi-governmental, networked, and completely governmental, some of which are mapped out in this case study. Interestingly, at least some of these structures can be understood as instances of multistakeholder governance.

The following is based on the experience of organizing a workshop in Tunis together with the Agence Tunisien d'Internet (ATI) in Tunis in 2013 on the Construction, Operation and Benefits of Internet Exchange Points, where the representatives of ten different IXPs spoke and shared their experiences running and governing an IXP. ${ }^{2}$ Several IXP governance structures are discussed in this case study as part of the wider debate on multistakeholder governance in Internet governance.

In particular, this paper studies three different IXP governance models that represent some of the largest and most influential IXPs in the world: 1) the DE-CIX in Frankfurt; 2) the CAIX in Cairo, Egypt; and 3) KIXP in Nairobi, Kenya. Each IXP shares a common function and common basic principles (enabling exchange of data), but they differ in regards to membership and governance structures. Hopefully, by taking a comparative approach to the governance of IXPs, it will be possible to better understand how multistakeholder governance is implemented in practice.

\section{A Brief Overview: What Are IXPs and Where Do They Come From?}

Internet Exchange Points (IXPs) are one of the main building blocks of the Internet. There are presently approximately 534 IXPs, more than half of which are in Europe and the United States. ${ }^{3}$

In simple terms, IXPs are a technical space where Internet service providers (ISPs) come together to link their different networks to each other. As has been noted elsewhere, the Internet is made up of a "network of networks" (Noam, 2001), and thus the points where those networks come together are of particular interest. The governance arrangements involving IXPs are interesting because they govern the core Internet infrastructure and in many cases have some traits of multistakeholder institutions.

\footnotetext{
${ }^{2}$ For further details see Internet Exchange Points-Construction, Operation and Benefits, Workshop organised in April 2013 in Tunis, Tunisia. Program available here: https://cihr.eu/wp-content/uploads/2014/06/TNV417TunisIXP.pdf.

${ }^{3}$ Packet Clearing House, Internet exchange point directory reports. Retrieved on November 8, 2014 from http://wwww.pch.net/ixpdir/summary.
} 
More specifically, ISPs and network operators need to exchange data, granting others access to their network. This can be done either by agreeing on a payment basis - for transit/upstream —or on a cost neutral basis, otherwise known as a peering agreement. In order for peering to work, IXPs offer a local network where any carrier, ISP, or network operator can connect and exchange traffic. The IXPs use switching equipment to build the local network by placing equipment in existing carrier neutral data centers and relying on dark fiber to connect those locations.

Over the last few years, IXPs have been established in many more areas and have in turn attracted greater Internet resources to those areas, ${ }^{4}$ decreasing the practice of "tromboning" traffic out of the region on long-haul routes. The availability of local points of exchange allows for more direct routing of traffic; by reducing the need to use long-distance transmission capacity for in-region traffic, it frees up existing long-haul capacity to handle out-of-region traffic.

Internet Exchange Points are the key locations defining traffic flows, as well as business and governance arrangements between Internet providers. That they have evolved as a leading part of the Internet is in no small part due to "norm entrepreneurs" like Packet Clearing House (PCH), an important enabler of IXP growth. The PCH is a non-profit research institute that supports operations and analysis in the areas of Internet traffic exchange, routing economics, and global network development. Its specific model of "neutral independent network interconnection" as embodied in most modern Internet Exchange Points has become the default standard for building Internet networks in large parts of the world (Ryan \& Gerson, 2012). As an organization, PCH travels the world and provides both hardware to private sector organizations in the process of starting Internet Exchange Points, as well as normative governance models on how IXPs should be governed. In particular, PCH activities promote a "community-oriented" IXP model within which IXPs act as "neutral hubs" providing services to their members rather than as for-profit companies. This model has become dominant in Europe and many parts of the world except the US, where "non-neutral" commercial ISPs remain the norm. PCH is extremely open in how it vigorously promotes this IXP model, stating on its website that:

PCH facilitates a multi-phase process that typically takes about six months: we organize local stakeholders in an un-served region to form an independent, not-for-profit industry association; guide that association through the necessary decisions regarding organizational, financial, and governance structure; work with government in the region to ensure a beneficial regulatory climate; assist them with site selection and preparation. ${ }^{6}$

There is strong agreement in the technical community that IXPs in and of themselves are a good thing, and that more IXPs are better (Franck, 2012). Together with greater international connectivity, IXPs are typically seen as reinforcing the decentralized structure, and thereby the resilience, of national Internet connectivity (Cowie, 2012). This is because having several hubs through which communications flow is more likely to be resilient when any one point fails, thereby reinforcing a network design strongly advocated by large parts of the technical community (Franck, 2012).

\footnotetext{
${ }^{4}$ Dates of establishment available at https://prefix.pch.net/applications/ixpdir/.

${ }_{6}^{5}$ See https://www.pch.net/home/index.php.

${ }^{6} \mathrm{See}$ https://www.pch.net/technology/operations.php.
} 
The PCH's efforts are flanked by that of the Internet Society (ISOC), another key Internet organization promoting a specific normative model of Internet governance. The ISOC also explicitly supports the development of IXPs and suggests that:

In many developing countries, poor connectivity between ISPs often results in the routing of local traffic over expensive international links simply to reach destinations within the country of origin. IXPs can also improve the quality of Internet services in a country by reducing the delays. IXPs also can be a convenient hub for hosting critical infrastructure within countries. ${ }^{7}$

At the same time Cisco Systems - the largest supplier of networking technology in the world - is one of the leading proponents of the creation of IXPs and often supports their development by donating networking equipment (Longwe \& Rulinda, 2005). As such, Cisco can be seen as a key enabler of IXP growth.

One of key basic parameters for Internet Exchange Points (IXPs) seems to be a low level of government regulation. This point is emphasized by Patrick Ryan and Jason Gerson, who argue that:

IXPs and networks tend to thrive when governments do not regulate their conduct in excess. IXPs and large commercial network operators would thus benefit from doing more to regulate themselves so governments do not feel the need to intervene. Good governance tends to emphasize neutrality and affordability, and we find that the most successful IXPs have adopted organizational policies that are robust and fair-policies that allow them to act without undue influence from individual ISPs (Ryan \& Gerson, 2012, p. 3).

Importantly, IXPs also enable a key technical and economic practice called "peering." In this model, Internet service providers (ISPs) and other large network providers agree to link their networks without charging each other (Chatzis et al., 2013; Sowell, 2012). As running a network involves considerable costs, this commitment is quite notable as any individual network owner could conceivably send large amounts of traffic across the other networks and thereby considerably raise the latter's costs. The agreement to peer through an IXP essentially takes certain business models off the table and thus entails the promotion of a specific economic model. In fact, an efficient market for connectivity has developed based on voluntary contractual agreements. This should be contrasted sharply with that of traditional regulated forms of voice traffic exchange, which are not based on voluntary contractual agreements and adopt legacy pricing.

Operating in a highly competitive environment, largely without central organization and strong regulatory regimes, the Internet model of traffic exchange has produced comparatively low prices. Peering agreements play an important role in this market. A survey of peering agreements gathered responses from 4331 ISP networks, representing $86 \%$ of the world's Internet carriers, incorporated in 96 countries, and these ISPs reported 142,210 peering agreements. Of these, 141,512 , or $99.51 \%$, were "handshake" agreements in which the parties agreed to commonly

\footnotetext{
${ }^{7}$ See http://www.internetsociety.org/what-we-do/issues/internet-exchange-points-ixps.
} 
understood terms without creating a written document. ${ }^{8}$ However, no matter how well developed this peering model is, it is not without problems. On a general level, "interconnection agreements are unseen in that there are no directly relevant statutes, there is no regulatory oversight, and there is little transparency." 9 More specifically, recent years have witnessed a rise in issues pertaining to peering agreements. For example, French telecom Orange and the American ISP Cogent experienced a conflict over their peering agreement when Orange lamented receiving a flow thirteen times greater than that it sent, in large part due to the fact that data from file-sharing website MegaUpload transited through Cogent. ${ }^{10}$ There have also been cases in which connectivity has been jeopardized following such matters (e.g., in Cogent vs. Sprint). ${ }^{11}$

IXPs provide a neutral space in which this specific technical and economic practice of no-cost exchange of data flows - "peering" - can take place. The typical agreements enabled through IXPs are so-called multilateral peering agreements, in which many networks meeting at an IXP join a single agreement rather than conclude separate bilateral arrangements. The average number of agreements reported was 32.8 for each of the entities in the survey. The distribution is long-tailed, with $62 \%$ of the respondents having ten or fewer agreements. It has therefore been possible to ensure global connectivity among two billion users by means of a relatively small number of agreements, less than $1 \%$ of a full mesh. ${ }^{12}$

As IXPs only allow such peering relationships among their members, they also create in essence a kind of "default contract" between networks wishing to connect. Despite the spread of such default contractual arrangements across the world, their role in creating the Internet as it exists today is only now beginning to become better understood (Chatzis et al., 2013).

\section{Studying IXP Governance}

IXPs are fascinating institutional spaces precisely because they are designed as "neutral" and "communal" spaces (ones that enable "peering," which is often described as a neutral practice). While this would seem to suggest some form of multistakeholder governance (de la Chapelle, 2007), the actual operational control and governance of IXP arrangements is extraordinarily diverse.

The following short case studies serve to illuminate the overall nature of IXP governance in the three different cases: 1) the DE-CIX in Frankfurt; 2) the CAIX in Cairo, Egypt; and 3) KIXP in Nairobi, Kenya. The governance model in each case is examined in detail, with a focus on how it relates to conceptions of multistakeholder governance.

\section{A. The German Internet Exchange (DE-CIX)}

The German Internet Exchange was founded in 1995 and has since developed into the largest Internet Exchange Point in the world. As a project founded by three German ISPs, it grew to

\footnotetext{
${ }^{8}$ D. Weller and B. Woodcock, "Internet Traffic Exchange: Market Developments and Policy Challenges," OECD Digital Economy Papers, No. 207 (OECD Publishing, 2013), http://dx.doi.org/10.1787/5k918gpt130q-en. Orange lamented receiving a flow thirteen times greater than it sent, in large part due to the fact that Megaupload data transited through Cogent.

${ }^{9}$ L. DeNardis, The Emerging Field of Internet Governance, cit., p. 567.

${ }^{10}$ For the 2012 decision, see http://www.autoritedelaconcurrence.fr/user/standard.php?id rub=418\&id article=1971

${ }^{11}$ P. Weiser, “The Future of Internet Regulation," University of Colorado Law Legal Studies Research Paper, No. 09-02 (2009).

${ }^{12}$ D. Weller and B. Woodcock, "Internet Traffic Exchange."
} 
accommodate over 600 customers (i.e., participants at the peering platform) by 2014. Notably, DE-CIX also operates IXPs in Hamburg, New York, and Dubai, giving it considerable global reach. ${ }^{13}$

The organization of DE-CIX itself was founded with the support of the trade association "eco," now also called the German Internet Association. DE-CIX has been institutionally attached ever since and is legally a complete subsidiary of the eco trade association. DE-CIX and eco share the same executive director, Harald A. Summa, and board members are elected by the members of the eco trade association. Apart from the private companies that are members of the eco trade association and eco staff, no other stakeholders have the ability to influence decisions made by eco about DE-CIX IXP. There is also very little transparency about such policy decisions, or about the exact relationship between DE-CIX and eco. Even on the DE-CIX Advisory Board, a consultative organ, one of the board members is allocated directly by eco, while DE-CIX customers elect the other four. The DE-CIX seems to maintain a model of strong corporate control. DE-CIX claims that it is neutral on its website, which reflects a conception of corporate stakeholderism not necessarily shared by other IXPs: "in order to be truly neutral, DE-CIX is entirely owned by eco-Association of the German Internet Industry-and is therefore "owned" by the Internet industry."

Similarly to in other countries, there has been an ongoing struggle between the incumbent Internet service provider in Germany, Deutsche Telekom, and the rest of the industry about the usage and role of the IXP. While almost all other Internet service providers in Germany have agreed to use DE-CIX and "peer" their traffic there, Deutsche Telekom has thus far refused, instead making individual private agreements with other network providers (Heise Online, 2013). Thus, the common normative space created by DE-CIX is explicitly contested by Deutsche Telekom, as is the norm of "peering."

More recently, DE-CIX has been brought into a considerable disrepute due to its complicity in German foreign Internet surveillance. The German BND has publicly "acknowledged tapping data flowing through a key Frankfurt Internet exchange point" (Preuschat \& Troianovski, 2014), which led to considerable public debate in Germany. However, DE-CIX was apparently unable to tell the public that its connection was under surveillance due to Germany's G-10 laws (Ermert, 2013). There are open questions as to how long this surveillance took place, whether eco or DECIX resisted attempts at surveillance, which traffic is still being monitored, and whether this type of mass surveillance is still on-going (Ermert, 2013).

When the overall structure of DE-CIX is observed, it seems relatively clear that it is operated, managed, and governed by the eco trade association, as well as the companies that are members of eco. Thus, a specific subset of the German private sector organized by eco has the exclusive ability to manage and govern a key Internet resource. Gerson and Ryan (2012) have suggested that DE-CIX should be considered a "managed non-profit [in which] DE-CIX thus views its ISPs as customers, not members or shareholders" (12). This seems to correspond to the vocabulary preferred by DE-CIX that, indeed, conveys that the "customer summits." ${ }^{14}$ Other actors, like the German government or BND, are able to regulate DE-CIX, but only by virtue of being part of the German government and an intelligence agency. They are not involved in the governance

\footnotetext{
${ }^{13}$ See https://www.de-cix.net/about/.

${ }^{14}$ See http://www.customersummit.net/home/.
} 
structures of DE-CIX per se and can only regulate from outside. The involvement of other stakeholders is neither possible nor expected.

As much as DE-CIX would wish to absolve themselves of responsibility for these problems, this remains a considerable challenge to both democratic and multistakeholder governance: many of the actors affected by the policy decisions and public statements (or lack of them) of DE-CIX and eco have no seat at the table. This lack of representation of key stakeholder groups such as civil society, the academic and technical communities, and even other branches of government in a crucial Internet institution seriously challenges the legitimacy of DE-CIX. While it is certainly a very well-functioning private company, it fails to live up to many of the claims of multistakeholder Internet governance.

\section{B. The Cairo Internet Exchange Point (CAIX) in Egypt}

The Cairo Internet Exchange Point (CAIX) was founded by the Egyptian Ministry of Communications and Information Technology (MICT) in 2002. As such, it is unusual among IXPs in that it was founded and is run by a public authority. This is interesting, because in certain cases the global IXP community has chosen not to recognize Internet Exchange Points that were not considered to be run following the standards of the global community. This was the case for Tunisia for many years, and while its technical infrastructure seemed to suggest an exchange point, it was not recognized as an Exchange Point by the IXP association Euro-IX until 2011. ${ }^{15}$ ISPs are typically allowed into the Euro-IX community by accepting certain basic standards and business models, most fundamentally the "peering" model, but also standards regarding nondiscrimination of customers. Thus, Euro-IX could be considered to be an exclusive club whose members decide which new members are allowed to join.

CAIX was founded in 2002 and initially only linked to two Internet service providers. Over time it gained more and more members, and today all of the large and medium sized ISPs in Egypt are part of the exchange. ${ }^{16}$ The governance of the Cairo Internet Exchange Point has also changed substantially, with authority transferred from the Ministry of Communications and Information Technology (MICT) to the National Telecommunications Regulatory Authority (NTRA). However, the NTRA board is chaired by the Minister of Communications and Information Technology, and includes representatives of the Council of State, the National Security Authority, the Ministries of Defense, Interior, and Finance, as well as a representative of the Radio and Television Union. ${ }^{17}$ Interestingly, this means that it is governed by a broader set of stakeholders than DE-CIX, including what could be considered private sector, government, and at least some "civil society" representatives. Despite the breadth of participation, the decisionmaking processes under CAIX lack transparency and it is very difficult to discern-based on the public information available- how key decisions about CAIX are made.

\footnotetext{
${ }^{15}$ Under the former regime, all ISPs were obliged to route their traffic via the ATI to facilitate internet filtering and surveillance. See https://www.euro-ix.net/ixps/370-TunIXP As of early 2013, however, a decision by the ICT Ministry to amend regulatory provisions resulted in Tunisiana and Orange Tunisie now being able to bypass the ATI for incoming and outgoing international internet traffic, see https://freedomhouse.org/report/freedomnet/2013/tunisia\#.VF5I6_TF-RN.

${ }^{16}$ See http://caix.net.eg/index.php/aboutcaix.

${ }^{17}$ See http://www.tra.gov.eg/english/DPages_DPagesDetails.asp?ID=175\&Menu=5.
} 
In an important case, the NTRA was directly implicated in the Internet and mobile phone shutdown during Tahrir Square protests in the spring of 2011. This at the very least raises questions about whether the NTRA can be considered an impartial and neutral governance authority for a neutral Internet Exchange Point in Egypt (Freedom House, 2012).

\section{Kenya Internet Exchange Point (KIXP)}

Kenya's Internet Exchange Point is particularly interesting because it has been held up as a leading example of multistakeholder Internet governance by US Assistant Secretary for Communications and Information, Lawrence Strickling, who is also the head of the US National Telecommunications and Information Administration (NTIA). Strickling has remarked: "where Africans have chosen the path of openness and inclusion-two key attributes of the multistakeholder process - the rewards have been great. Take the example of the Kenya Internet Exchange Point" (Hutty, 2011). These remarks suggest KIXP is a potential model of multistakeholder governance. So how was KIXP founded and how is it governed in practice?

The inception of the IXP can be traced back to a Kenyan network engineer attending an Internet Society networking workshop for developing countries in San Jose, California, US (Longwe \& Rulinda, 2005). After obtaining support in the form of free networking equipment from Cisco Systems and funding from the United Kingdom's Department for International Development and the Telecommunications Service Providers of Kenya (TESPOK), it was possible to found KIXP in 2001. Interestingly at the time there was considerable mistrust between Internet Service Providers (ISPs) - the main future clients of KIXP — with some refusing to collaborate and others fearing such an institution would be misused for "undue and possibly unethical advantage" if hosted by a competitor (Longwe \& Rulinda, 2005).

Despite these trust and collective action problems, KIXP was created on "neutral" ground in 2001, only to be engaged almost immediately in a legal battle with the incumbent telephone \& Internet provider, Kenya Telecom, over licensing issues (Longwe \& Rulinda, 2005). Such conflicts between IXPs and incumbents are not uncommon, and similar problems can be observed in Tunisia and Germany, where telecommunications incumbent support for IXPs is highly limited.

Following a vigorous response from all other Internet Service Providers (ISPs) in Kenya, KIXP was eventually granted a license to operate, which they continue to hold to this day. The local trade association TESPOK governs KIXP, although it is unclear based on publically available information how the board of directors is appointed and how it makes decisions. It seems that similar to DE-CIX, governance of KIXP takes place within the TESPOK trade association, which includes most telecommunications service providers in Kenya. Notably no additional stakeholders, such as civil society, academia, government, or international organizations, are involved in the governance or day-to-day operations of KIXP. Thus it could be argued that KIXP was set up with multistakeholder coordination under the leadership of the private sector, but the day-to-day operations and governance of KIXP are entirely in the control of a private sector trade association.

While this makes NTIA Director Strickling's endorsement of KIXP as a model of multistakeholder governance very puzzling, it is possible that this is exactly what he meant. Private sector governance and management of systems is indeed by far the most prominent example of multi-stakeholder governance, and in most cases the default. While this seems 
perfectly reasonable for the day to day management of systems, the larger governance questions surrounding IXPs - for example, the German intelligence agency BND tapping DE-CIX - raise questions about who the stakeholders are and who should be involved. It also raises the question of what is meant by "the path of openness and inclusion-two key attributes of the multistakeholder process" (Lawrence Strickling, in Hutty, 2011).

\section{Conclusion}

Internet Exchange Points (IXPs) are a fascinating and under-researched part of the modern Internet. From the perspective of the social sciences, IXPs are also extraordinary places for promoting highly plural and diverse local governance models, while still keeping a core of common norms and principles. In this sense, Internet Exchange Points are an excellent place to study institutional development in a globalized world. Following the excellent comparative governance analysis by Ryan and Gerson (2012), this paper attempts to provide an additional critical institutional analysis of IXPs in an international context.

It seems relatively clear that the core international "norm setters" or "enablers" of international IXP governance can be found at the Packet Clearing House and the Internet Society. These organizations have extensively promoted the core "peering" model over the past two decades and have thus considerably contributed to its spread across the world. By providing expertise and access to free networking equipment, both organizations are well positioned to support the internationalization of these institutions.

At the core of an IXP are the peering relationships between the organizations that connect to it. IXPs constitute a common pool resource and neutral ground in which competing private actors agree to collaborate rather than compete. While this would seem to fly in the face of any modern national competition policy, it actually seems to stimulate Internet growth in some areas (Chatzis et al., 2013). This development is then supported by other institutions like Euro-IX, which provides a kind of club accreditation system which only IXPs are able to join and can exchange experiences in regular meetings.

Moreover, once some commercial partners began following this model, it quickly spread and became the default model for cooperation among networks. In this context it doesn't seem to matter whether the IXP is publicly or privately governed, or whether it is non-profit or for-profit, as long as it fosters the principle of "peering" and sees to its members' needs in a way that can be considered effective. Why legal form seems to not matter much in the context of the questions asked in this case study is unclear, and further research will be required to develop a compelling answer.

Thus the actual commonality of governance forms between IXPs is actually very small. It is perhaps this "non-political" nature of IXP governance that has made the IXP model so successful, and caused IXPs to spread across the world. In other words, the commonality of IXP governance is not their internal governance or legal status - which may differ substantially_but their promotion of peering and output oriented effectiveness. This also implies that looking at the inclusiveness of these governance structures may not be key to understanding their perceived legitimacy in the global Internet community. From a multistakeholder perspective, many potential stakeholders may be left out, from governments to end-users. The perceived legitimacy seems to be more closely linked to the efficacy and efficiency of IXPs. This considerably limits 
their inclusiveness, which is perhaps not unreasonable given that so few people know what an IXP is in the first place.

What is consistent, however, across the cases is the conflict with the incumbent operator, typically a former state monopolist in the country where the IXP is based. These actors normally have little interest in the principle of "peering," and their resistance to the IXP model is evident in Germany and Kenya, as well as other cases not discussed here, such as Mexico. Ryan and Gerson note that: "Mexico has been unable to develop a successful IXP due to incumbents' unwillingness to cooperate" (2012, pp. 22-23). This suggests that IXPs do not just promote a "neutral" collaboration model, but are likely to promote competition between networks and may also help to open markets. While this may be a positive development for the Internet as a whole, it may not be in the commercial interests of incumbent Internet providers and indeed of states wishing to protect some form of communications monopoly.

Where then is multistakeholder governance left in all of this? Multistakeholderism is generally guided by the idea that the inclusion of those affected by, or who affect the outcome, is good for achieving the process' stated objectives. However, this is not always the case: the viability of a multistakeholder process can also be determined by its effectiveness. In the case of IXPs, this seems to be the guiding principle, as their primary objective is to lower the costs of transit and reduce routing time. If this is the goal, then focusing on efficiency rather than inclusiveness seems reasonable. If inclusiveness is to be reached, then perhaps the scope of the objectives need to be broadened. Since there is no single uniform and accepted definition of what a "stake" might involve (e.g. interest, legal obligation, moral right) or who can be said to have one, defining "stakeholders" is also a problem. Stakeholders can be such because they are seen to have some legitimate claim in the operations of the institution because they are affected by its actions, or because they subjectively perceive themselves to be stakeholders. In this sense, perhaps a warning would be appropriate: the very conception of "stakeholder" in this study is left rather general so as to encompass many different views.

To a certain degree, the public sector was involved in some of the Internet Exchange Points mentioned here. Through legislation, the public sector is occasionally also involved in regulating and thereby governing IXPs, as is evident in both the German and Kenyan experience. However, it is notable that civil society and even large parts of the private sector outside of the Internet economy have been so far entirely unable to have a stake in IXPs. While this seems perfectly reasonable in regard to the day-to-day operations and management of an IXP, it seems more difficult to justify when these external stakeholders are equally affected as those within the process, and yet have no influence over important policy changes.

It seems evident that IXPs are part of what leading proponents consider multistakeholder governance. It is not uncommon for multistakeholder governance arrangements - when studied more closely - to end up looking a lot like private coordination, common-pool resources, or private clubs (Wagner, 2013). This does not necessarily have to be the case. For example, the Top-IX Internet exchange point in Turin seems to be an interesting example of multi-stakeholder governance with greater levels of transparency in its decision-making processes than the examples discussed here. ${ }^{18}$ While further research is required to properly verify this claim, initial

\footnotetext{
${ }^{18}$ For further details, see http://www.top-ix.org/en/consorzio/ for further details.
} 
conversations with those involved in the governance of Top-IX suggest that a far broader set of stakeholders are involved.

Finally there are serious human rights issues raised by the misuse of IXPs for mass surveillance, national censorship, or the mass disconnection of Internet communications. These issues have arisen in different countries across the world, such as Germany, Tunisia, and Egypt. The absence of influence by stakeholders affected by this misuse seems to be part of what is creating the problem.

This again does not necessarily mean that expanding the multistakeholder governance of IXPs is the answer to these problems; however, it can reasonably be suggested that more inclusive governance of IXPs could have helped prevent - or at least mitigate - many of the human rights concerns that IXPs raise. Importantly, however, broad claims that IXPs inherently follow "the path of openness and inclusion" (Lawrence Strickling in Hutty, 2011) do not provide the full picture. Instead, further research is required to fully understand the governance of IXPs and their role in promoting development, growth, and trust in the Internet ecosystem, as well as a far more critical analysis of their governance structures. 


\section{Bibliography}

Chatzis, N., Smaragdakis, G., \& Feldmann, A. (2013). On the importance of Internet eXchange Points for today's Internet ecosystem Categories and Subject Descriptors. Cryptome.org.

Cowie, J. (2012). Could It Happen In Your Country?-Renesys. Renesys. Retrieved July 31, 2014, from http:/www.renesys.com/2012/11/could-it-happen-in-your-countr/.

De la Chapelle, B. (2007). Multi-Stakeholder Governance-Emergence and Transformational Potential of a New Political Paradigm. In D. Helbing (Ed.), Managing complexity: insights, concepts, applications. Springer.

Ermert, M. (2013). NSA-Abhörskandal PRISM: Internet-Austauschknoten als Abhörziele. heise online. Retrieved August 19, 2014, from http://www.heise.de/newsticker/meldung/NSAAbhoerskandal-PRISM-Internet-Austauschknoten-als-Abhoerziele-1909604.html.

Franck, P. (2012). Peter Franck zu Freiheit und Sicherheit im Netz. eco e.V. Verband der deutschen Internetwirtschaft. Retrieved from http://www.eco.de/2012/news/peter-franck-zufreiheit-und-sicherheit-im-netz.html.

Freedom House. (2012). Egypt. In S. Kelly, S. Cook, \& M. Truong (Eds.), Freedom on the Net. Washington D.C.: Freedom House.

Heise Online. (2013). Das DE-CIX und das "Schland-Netz": Betreiber empört über TelekomPläne zum "Schengen-Routing." heise online. Retrieved August 19, 2014, from http://www.heise.de/netze/meldung/Das-DE-CIX-und-das-Schland-Netz-Betreiber-empoertueber-Telekom-Plaene-zum-Schengen-Routing-2044731.html.

Hutty, M. (2011). US places Internet Exchanges at centre of Internet Governance debate. LINX Public Affairs. Retrieved August 19, 2014, from https://publicaffairs.linx.net/news/?p=5446.

Longwe, B., \& Rulinda, C. (2005). Of Gateways and Gatekeepers: The History of Internet Exchange Points in Kenya and Rwanda. In F. Etta \& L. Elder (Eds.), AT THE CROSSROADS: ICT Policymaking in East Africa. Nairobi, Kenya: East African Educational Publishers/IDRC 2005.

Noam, E. (2001). Interconnecting the network of networks. Cambridge: MIT Press.

Preuschat, A., \& Troianovski, A. (2014). German Intelligence Admits to Frankfurt E-Mail TapDigits-WSJ. Wall Street Journal. Retrieved August 19, 2014, from http://blogs.wsj.com/digits/2013/10/09/german-intelligence-admits-to-frankfurt-e-mail-tap/.

Ryan, P. S., \& Gerson, J. (2012). A Primer on Internet Exchange Points for Policymakers and Non-Engineers. SSRN Electronic Journal. doi:10.2139/ssm.2128103.

Sowell, J. (2012). Empirical studies of bottom-up Internet governance. In Telecommunications Policy Research Conference (TPRC) (pp. 1-31). Washington D.C.: George Mason University.

Wagner, B. (2013). Governing Internet Expression: The International and Transnational Politics of Freedom of Expression. European University Institute. 


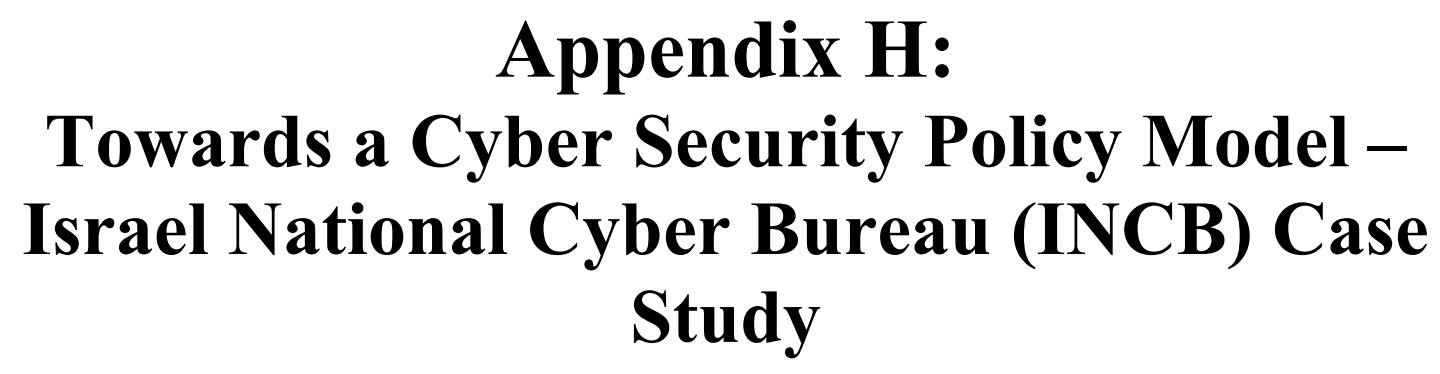




\title{
NoC Internet Governance Case Studies Series: Towards a Cyber Security Policy Model - Israel National Cyber Bureau (INCB) Case Study
}

\author{
Daniel Benoliel \\ Haifa Center of Law and Technology, University of Haifa Faculty of Law
}

Editorial Note: Context, Character, and Purpose of the Case Study

This case study is part of a globally coordinated, independent academic research pilot project by the Global Network of Interdisciplinary Internet \& Society Research Centers (NoC). Facilitated by the Berkman Center for Internet \& Society at Harvard University, this study examines existing multistakeholder governance groups with the goal of informing the future evolution of the Internet governance ecosystem. Building upon the NETmundial Principles and Roadmap, it contributes to current policy debates at the international level, including the Internet Governance Forum, the NETmundial Initiative, and other organizations and efforts.

Internet governance is an increasingly complex concept that operates at multiple levels and in different dimensions, making it necessary to have a better understanding of both how multistakeholder governance groups operate and how they best achieve their goals. With this need in mind, at a point where the future of Internet governance is being re-envisioned, colleagues from several NoC institutions around the world have written twelve case studies examining a geographically and topically diverse set of local, national, and international governance models, components, and mechanisms from within and outside of the sphere of Internet governance. Key findings from these cases are summarized in a synthesis paper, which aims to deepen our understanding of the formation, operation, and critical success factors of governance groups and even challenge conventional thinking.

The research, based on twelve case studies, suggests that there is no single best-fit model for multistakeholder governance groups that can be applied in all situations. Rather, it reveals a range of approaches, mechanisms, and tools available for both the formation and operation of such groups. The analysis demonstrates that the success of governance groups depends to a large degree on the careful selection, deployment, and management of suitable instruments from this "toolbox." As governance groups pass through different phases of operation, conveners and facilitators must remain alert to changes in circumstances that necessitate adjustments to the approaches, mechanisms, and tools that they deploy in order to address evolving challenges from inside and outside. This case study provides insights into how those instruments can be deployed and adjusted over time within such groups, and highlights how their interactions with important contextual factors may be successfully managed within given resource restraints.

The research effort is grounded in a diversity of global perspectives and collaborative research techniques. Adhering to objective and independent academic standards, it aspires to be useful, actionable, and timely for policymakers and stakeholders. More broadly, the Network of Centers seeks to contribute to a more generalized vision and longer-term strategy for academia regarding its roles in research, facilitation and convening, and education in and communication about the Internet age.

For additional information on the initiative, please contact Urs Gasser, Berkman Center for Internet \& Society, at ugasser@cyber.law.harvard.edu. 


\begin{abstract}
This case study examines the elements of the creation of a cyber security policy model at a national level. It uses Israel's recently established National Cyber Bureau (INCB) cyber command funneled by its national cyber policy as a case in point. In so doing the brief offers a cross-section comparison between leading cyber security national policies of the United States, United Kingdom, Canada, Japan and the Netherlands. It further introduces comparable policies including the balancing of cyber security with civil liberties, cyber crime policy, adherence to international law and international humanitarian law, forms of regulation (technological standards, legislation, courts, markets or norms) and prevalent forms of cooperation (intra-governmental, regional, public-private platform (PPP) and inter-governmental cooperation).
\end{abstract}




\section{Table of Contents}

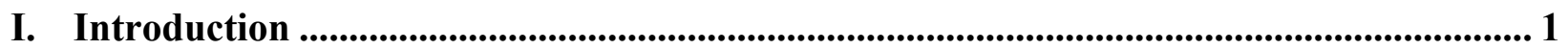

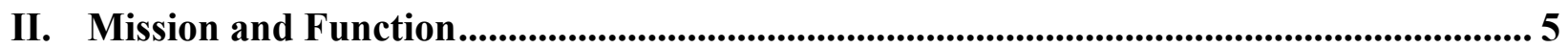

III. The Positive Framework ................................................................................. 10

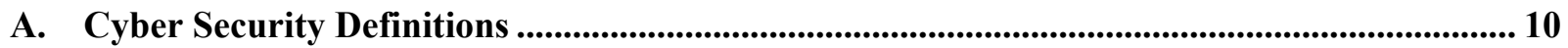

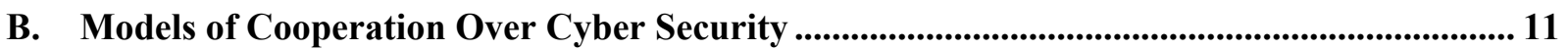

1. Inter-Governmental Cooperation ................................................................................... 11

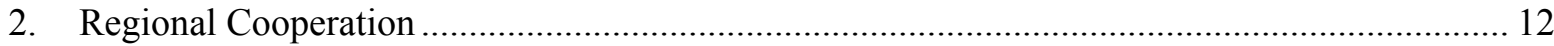

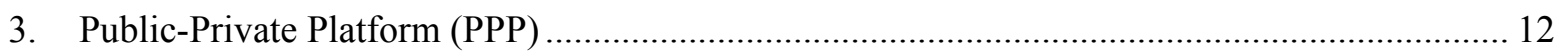

4. Economics of Information Security Considerations ............................................................... 13

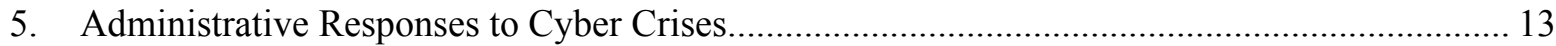

6. Linking International Cyber Security Policy to Domestic Law ……………………………..... 13

IV. A Cross-Section Comparison ........................................................................................ 14 


\section{Introduction}

This policy brief takes a descriptive approach, describing and explaining the characteristics and behaviors of chief stakeholders in Israel's cyber security policy, including the Israeli government, the recently established Israeli National Cyber Bureau (INCB), ${ }^{1}$ and secondary stakeholders - the intermediaries indirectly intertwined with Israel's cyber policy - such as local academia and cyber industry. On a broader scale of relevancy this policy brief offers a comparison of Israel's cyber security policy with the advanced cyber security policies of additional countries. Lastly, this brief describes the cyber security standpoints of multiple international stakeholders, including the NETMundial network as well as other related NGOs, standard setting organizations (SSOs), and international organizations such as the United Nations, NATO, and the European Union.

Recent revelations about the United States National Security Agency's (NSA) clandestine mass electronic surveillance data mining projects raised a public debate worldwide over the legality of governmental compliance with democratic principles. ${ }^{2}$ From a legal policy perspective, designing the nooks and crannies of cyber security is challenging for two decisive reasons. First, the field is largely shrouded with secrecy and over classification. Second, the traditional major stakeholders in the field are national defense and intelligence organs.

This excessive secrecy in the Israeli case and elsewhere is already burdensome in current policy initiatives. ${ }^{3}$ Not surprisingly, the original attempts to regulate cyber security for the private sector started with - and are still predominantly restricted to-technological standard setting and governmental-industry cooperation therein. To date, four such endeavors are most prevalent. These are the highly popular International Organization for Standardization's (ISO) standards ISO 27001 , which appeared as early as $2005,{ }^{4}$ and ISO $27002^{5}$ - two cyber security standards offering ISO/IEC voluntary certifications for complying businesses. In addition, one should mention the Information Security Forum's (ISF) Standard of Good Practice for Information Security (SoGP), which covers a spectrum of information security arrangements to address

\footnotetext{
${ }^{1}$ This descriptive approach corresponds with Donaldson \& Preston's Multistakeholder theory. See Thomas Donaldson, and Lee Preston, "The Stakeholder Theory of the Corporation: Concepts, Evidence, and Implications," Academy of Management Review vol. 20(1) 70 (1995) (arguing that the theory has mutually supportive facets, which are descriptive, instrumental, and normative).

${ }^{2}$ A key example is the PRISM project. Prism gathers Internet communications derived from demands made to Internet companies such as Yahoo!, Inc. It does so under Section 702 of the FISA Amendments Act of 2008 in order to yield any data that counterparts court-approved search terms. See Barton Gellman and Ashkan Soltani, "NSA infiltrates links to Yahoo, Google data centers worldwide, Snowden documents say," The Washington Post, October 30, 2013.

${ }^{3}$ Lior Tabansky, The Chair of Cyber Defense, "Yuval Ne'eman Workshop for Science, Technology and Security Tel Aviv University Israel," Article no III.12 (January 2013), at 2, http://sectech.tau.ac.il/sites/default/files/publications/article_3_12_-_chaire_cyberdefense.pdf. In an interview with Mr. Tal Goldstein (on file with author) from the Israeli National Cyber Bureau on 21 September 2014, it was further emphasized that to a large extent commercial enterprises themselves withhold their cooperation with INCB cyber defense organs due to commercially-related secrecy concerns.

${ }^{4}$ ISO, “An Introduction To ISO 27001 (ISO27001)," http://www.27000.org/iso-27001.htm (described as "specification for an information security management system (ISMS)").

${ }^{5}$ ISO, "Introduction To ISO 27002 (ISO27002)," http://www.27000.org/iso-27002.htm (offering "guidelines and general principles for initiating, implementing, maintaining, and improving information security management within an organization").
} 
business risks associated with information systems, ${ }^{6}$ the Software Assurance Maturity Model (SAMM) best practices in software security, ${ }^{7}$ and lastly the Cloud Security Alliance (CSA) best practices for cloud computing. ${ }^{8}$ Against this backdrop of technical orientation towards cyber security, the focus has gradually shifted onto other stakeholders interested in Internet governance. Such stakeholders typically preside within academia, international nongovernmental initiatives, and governments.

To date, numerous governments have already taken on this initiative while offering the most advanced sets of cyber security policies. These are noticeably the United States, ${ }^{9}$ the United Kingdom, ${ }^{10}$ Canada, ${ }^{11}$ Japan, ${ }^{12}$ Germany, ${ }^{13}$ the Netherlands, ${ }^{14}$ and Israel's establishment of an Israel National Cyber Bureau (INCB) in 2011. These national initiatives have also served to establish cyber security threats as predominantly national rather than merely global or

\footnotetext{
${ }^{6}$ See The Information Security Forum (ISF), "Standard of Good Practice for Information Security (SoGP)," https://www.securityforum.org/tools/sogp/.

${ }^{7}$ The Software Assurance Maturity Model (SAMM) is an open framework to help organizations formulate and implement a strategy for software security. The building blocks of the model are the three maturity levels defined for each of the twelve security practices. These define a wide variety of activities in which an organization could engage to reduce security risks and increase software assurance. Common Assurance Maturity Model (CAMM), "Software Assurance Maturity Model: A guide to building security into software development Version—1.0," at 3, http://www.opensamm.org/downloads/SAMM-1.0.pdf.

${ }^{8}$ Cloud Security Alliance (CSA), "Security Guidance for Critical Areas of Focus in Cloud Computing," (3rd ed., 2011), https://downloads.cloudsecurityalliance.org/initiatives/guidance/csaguide.v3.0.pdf. The CSA's best practices cover potential legal issues when using cloud computing. These include protection requirements for information and computer systems, security breach disclosure laws, regulatory requirements, privacy requirements, international laws, etc.

${ }^{9}$ See generally, Barack Obama, "Executive Order-Improving Critical Infrastructure Cybersecurity," February 12, 2013; The White House, "Presidential Policy Directive - Critical Infrastructure and Resilience," February 12, 2013 (PDD-21); H.R. 3696, 13th Congress 1st Session, "National Cybersecurity and Critical Infrastructure Protection Act of 2013"; U.S Department of Homeland Security, "NIPP 2013: Partnering for Critical Infrastructure Security and Resilience," Barack Obama, "International Strategy For Cyberspace: Prosperity, Security, and Openness in a Networked World," The White House (May 2011); National Infrastructure Advisory Council, "Critical Infrastructure Partnership Strategic Assessment: Final Report and Recommendations" (October 14, 2008); The White House, "Homeland Security Presidential Directive 7: Critical Infrastructure Identification, Prioritization, and Protection," (December 17, 2003) (hereafter "HSPD-7"); The White House, "Presidential Decision Directive/NSC63," (May 22, 1998); White House, "Presidential Decision Directive 63: Policy on Critical Infrastructure Protection" (1998); The President's Commission on Critical Infrastructure Protection (PCCIP), "Critical Foundations: Protecting America's Infrastructures," Washington, October 1997. PCCIP does not exist today. Its functions have been reallocated per HSPD-7.

${ }^{10}$ For Great Britain's 2009 policy initiative, see UK Cabinet Office, Cyber Security Strategy of the United Kingdom: Safety, security and resilience in cyber space (London: The Cabinet Office, CM 7642, June 2009), http://www.official-documents.gov.uk/document/cm76/7642/7642.pdf.

${ }^{11}$ See Government of Canada, Canada's Cyber Security Strategy for a Stronger and More Prosperous Canada, (2010), http://www.publicsafety.gc.ca/cnt/rsrcs/pblctns/cbr-scrt-strtgy/cbr-scrt-strtgy-eng.pdf.

${ }^{12}$ Information Security Strategy for protecting the nation (2013). Earlier the Japanese Information Security Policy Council released a strategy. See Information Security Strategy for Protecting the Nation, (May 11, 2010), http://www.nisc.go.jp/eng/pdf/New_Strategy_English.pdf.

${ }^{13}$ See Federal Ministry of the Interior, Cyber Security Strategy for Germany (February 2011), https://www.bsi.bund.de/SharedDocs/Downloads/EN/BSI/Publications/CyberSecurity/Cyber_Security_Strategy_for Germany.pdf?_blob=publicationFile.

${ }^{\overline{14}}$ See National $\overline{C y}$ ber Security, "Strategy 2: From awareness to capability" (2013). See also Ministry of Science and Justice, The National Cyber Security Strategy (NCSS), The Ministry of Security and Justice, the Netherlands (2011).
} 
international issues. ${ }^{15}$ This policy brief focuses on the national level within this natural regulatory flow.

Other stakeholders have also begun initiating equivalent policies. To mention but a few, the NETmundial platform noticeably offers a vibrant bottom-up NGO-based alternative. ${ }^{16}$ This platform directly establishes security as one of its seven principles for Internet governance: "Security, stability and resilience of the Internet should be a key objective" and elsewhere "Effectiveness in addressing risks and threats to security and stability of the Internet depends on strong cooperation among different stakeholders." 17 Similarly, the Organization for Security and Cooperation in Europe (OSCE) has been discussing cyber security issues for numerous years, offering yet another multinational discussion platform. To illustrate, at the OSCE Summit held in 2010 in Astana, Kazakhstan, the Heads of State and Government of the 56 participating States of the OSCE underlined that "greater unity of purpose and action in facing emerging transnational threats" must be achieved, while proposing an international "security community." "The Astana Commemorative Declaration mentions cyber threats as one of the significant emerging transnational threats that bridges the north-south divide between developed and developing countries. ${ }^{19}$ Yet the OSCE Summit has not yielded more concrete cyber security recommendations to date.

Lastly, a landmark decision recently took place at the United Nations (UN). For the first time in 2013 a group of governmental experts from fifteen member states agreed to acknowledge the full applicability of international law and state responsibility to state behavior in cyberspace. ${ }^{20}$ That is, by extending traditional transparency and confidence-building measures, and by recommending international cooperation making information and communications technology (ICT) infrastructure more secure against cyber threats worldwide. However, the decision has not yet become international law and is still nonbinding.

\footnotetext{
${ }^{15}$ Brigid Grauman, Cyber-Security: The Vexed Question of Global Rules: An Independent Report on CyberPreparedness around the World, edited by Security \& Defence Agenda (SDA) and McAfee Inc. Brussels: Security \& Defence Agenda (SDA), 2012 [hereafter, "the Security \& Defence Agenda (SDA) report"], at 66-67.

${ }^{16}$ The NETmundial platform is a voluntary bottom-up, open, and participatory process involving thousands of people from governments, private sector, civil society, technical community, and academia worldwide on Internet governance ecosystem. See Geneva Internet Platform, "GIP Exclusive Coverage of NETmundial," http://giplatform.org/events/netmundial.

${ }^{17}$ NETmundial Multi stakeholder Statement (April, 24th 2014), at 5, http://netmundial.br/wpcontent/uploads/2014/04/NETmundial-Multistakeholder-Document.pdf (defined as one of NETmundial's seven principles, titled: "Security and stability and resilience of the Internet"). The statement is a result of NETmundial's first conference held in Sao Paulo, Brazil between 23-24 April 2014.

NETmundial's “Roadmap for the Further Evolution of the Internet governance ecosystem" Part (2)III(1)(a) (titled: "Security and stability") in part (2) dealing with specific Internet governance topics - further reiterates international cooperation "on topics such as jurisdiction and law enforcement assistance to promote cyber security and prevent cybercrime." See NETmundial, "Roadmap for the Further Evolution of the Internet Governance Ecosystem," http://content.netmundial.br/contribution/roadmap-for-the-further-evolution-of-the-internet-governanceecosystem/177.

${ }^{18}$ The Astana Commemorative Declaration: Towards a Security Community (3 December 2010), http://www.osce.org/cio/74985?download=true.

${ }^{19}$ Id. at Article 9.

${ }^{20}$ See UN General Assembly, Group of Governmental Experts on Developments in the Field of Information and Telecommunications in the Context of International Security, A/68/98, June 24, 2013.
} 
The issue of information security has been on the United Nations agenda since the Russian Federation first introduced a draft resolution in the First Committee of the UN General Assembly in $1998 .^{21}$ Since then, there have been annual reports by the Secretary-General to the General Assembly with the views of UN member states on these issues. There have also been three Groups of Governmental Experts (GGE) that have reviewed present and future cyber threats and cooperative measures. $^{22}$

Against the backdrop of these initiatives, this policy brief offers a comparative review of Israel's National Cyber Bureau (INCB), established in 2011. The brief may ultimately assist in constructing a comprehensive national cyber security policy model partially based on Israel's example, as well as those of the United States, United Kingdom, Canada, Japan, and the Netherlands.

One question remains: why Israel? Two significant reasons come to mind. First, Israel's cyber defense apparatus is world-renowned and considered one of the top programs globally. To illustrate, an international comparative study of twenty-three developed countries put together by Brussels-based think-tank Security \& Defense Agenda (SDA) as part of its cyber-security initiative recently awarded Israel with a top grade on "cyber defense," alongside Sweden and Finland. ${ }^{23}$ Yet unlike these two Scandinavian countries, Israel sees approximately one thousand cyber attacks every minute. ${ }^{24}$ Second, according to Israel's National Cyber Bureau, Israel remarkably exports more cyber-related products and services than all other nations combined, apart from the United States. ${ }^{25}$ Both its technological prominence and this global market dominance have turned Israel into a global leader in the field and an excellent evolving working example.

Part A introduces the Israeli National Cyber Bureau initiative and the Israeli government's underlying recommendations. Part B offers a cross-sectional policy comparison between five leading national cyber security policies: those of the United States, United Kingdom, Canada, Japan, and the Netherlands. Finally, the conclusion identifies observations from the comparison of different national approaches, with a specific focus on the facilitation of academiagovernment cooperation in cyber security policy models.

\footnotetext{
${ }^{21}$ The General Assembly Resolution was adopted without a vote as A/RES/53/70.

${ }^{22}$ A first successful GGE report was issued in 2010 (A/65/201). In 2011 the General Assembly unanimously approved a resolution (A/RES/66/24) calling for a follow-up to the last GGE. See, The UN Office for Disarmament Affairs (UNODA), Fact sheet, Developments in the Field of Information and Telecommunications in the Context of International Security, http://www.un.org/disarmament/HomePage/factsheet/iob/Information_Security_Fact_Sheet.pdf.

${ }^{23}$ The Security \& Defense Agenda (SDA), "Cyber-Security: The Vexed Question of Global Rules," Report, (30 January 2012), 66-67.

${ }^{24} I d$. at 66. In fact, different to the experience of most countries with advanced cyber security policies, Israel's one did not evolve in response to civil threats i.e., cyber crime but instead it reacted mostly to national security considerations due to the country's notable geo-political security challenges. See also Interview with Mr. Tal Goldstein from the Israeli National Cyber Bureau, supra note 2.

${ }^{25}$ See Barbara Opall-Rome, "Israel Claims \$3B in Cyber Exports; 2nd Only to US," DefenseNews, June 20, 2014, http://www.defensenews.com/article/20140620/DEFREG04/306200018/Israel-Claims-3B-Cyber-Exports-2nd-OnlyUS (last year Israel sales reached $\$ 3$ billion which make approximately 5 percent of the global market).
} 


\section{Mission and Function}

Israeli cyber security policy was established based on two major official milestones. The first was the 2010 "National Cyber Initiative," which aimed for Israel to become a top five global cyber superpower by 2015 . $^{26}$ The second milestone, coming after years of departmental activities in various branches, was the Government of Israel's Government Resolution No. 3611 as of August 7, 2011, which adopted recommendations for the "National Cyber Initiative."27

At the core of these two initiatives stood the establishment of the Israel National Cyber Bureau (INCB) in the Prime Minister's office, reporting directly to the Prime Minister. ${ }^{28}$ The Bureau's mission is to serve as an advisory body for the Israeli Prime Minister, the government, and its committees on national policy in the cyber field and to promote implementation of such policies. $^{29}$ The decision to create the INCB ended a year-long struggle among different government stakeholders, including the Israel Security Agency (Shin Bet), vying for responsibility over the country's cyber security apparatus. ${ }^{30}$

The National Cyber Bureau's mandate is threefold. The first is to defend national infrastructures from cyber attack. ${ }^{31}$ This aspect is not restricted to traditional law enforcement deterrence approaches, as it also includes preventative measures. ${ }^{32}$ The second mandate is advancing Israel

\footnotetext{
${ }^{26}$ See The State of Israel, Ministry of Science and Technology, the National Council on Research and Development and the Supreme Council on Science and Technology, eds., "National Cyber Initiative_-Special Report for the Prime Minister" (2011) (Hebrew).

${ }^{27}$ In this backdrop the Israeli government has sought to establish a national cyber policy as soon as 2002 . In the same year Israel drew a list of 19 major infrastructures incorporating power production, water supply or banking, held as either public and private with purpose of standardizing core, albeit effectively limited legal and technological protection thereof. Id., at 67. Until the establishment of the Israeli National Cyber Bureau in 2011, Israel based its rather fragmented policies on Special Resolution B/84 on "The responsibility for protecting computerized systems in the State of Israel" by the ministerial committee on national security of December 11, 2002, launched the national civilian cyberdefense policy. In balance, it has been the latter Special Resolution that catalyzed the establishment of the Israeli Cyber Bureau. See Lior Tabansky, supra note 1, at 2. Israel undertook numerous other steps to address cyber threats. In 2002 a government decision established the State Authority for Information Security (Shabak unit). The Authority is accountable for the specialized guidance of the bodies under its accountability in terms of essential computer infrastructure security against threats of terrorism and sabotage. To illustrate, when the instigation of the biometric database in Israel led to an enormous public dispute, a recent law was enacted in 2009 and consequently the State Authority for Information Security received a defensive role in prevention of cyber attacks on the biometric database.

${ }^{28}$ Government of Israel passed Government Resolution No. 3611, titled: Advancing National Cyberspace Capabilities, Resolution No. 3611 of the Government of August 7, 2011, http:/www.pmo.gov.il/English/PrimeMinistersOffice/DivisionsAndAuthorities/cyber/Documents/Advancing\%20N ational\%20Cyberspace\%20Capabilities.pdf (for the non-official English version). See generally, also the State of Israel Prime Minister's Office-The National Cyber Bureau, http://www.pmo.gov.il/.

${ }^{29}$ Addendum A, section 1 (titled "Bureau Mission") states: "The Bureau functions as an advising body for the Prime Minister, the government and its committees, which recommends national policy in the cyber field and promotes its implementation, in accordance with all law and Government Resolutions." Id.

${ }^{30}$ See Jerusalem Post, Herb Keinon, Gov't to establish upgraded cyber security authority (21 September 2014).

${ }^{31}$ Id. Resolution No. 3611, at 1 ("To improve the defense of national infrastructures which are essential for maintaining a stable and productive life in the State of Israel and to strengthen those infrastructures, as much as possible, against cyber attack").

${ }^{32}$ On the immense challenges facing a traditional law enforcement reactive cyber security deterrence, see National Research Council, Proceedings of a Workshop on Deterring Cyberattacks (Washington, DC: National Academies Press, 2010); Martin C. Libicki, Cyberdeterrence and Cyberwar (Santa Monica, CA: RAND, 2009). But see, Derek
} 
as a world-leading center of information technology based on the country's high technological advantage. ${ }^{33}$ The third mandate is to encourage cooperation between academia, industry and the private sector, government offices and the security community, respectively. ${ }^{34}$

These broad policies were further detailed within the Israel's government Resolution No. 3611 in three ways. The Resolution's first mentioned decision and its raison d'être was officially establishing a National Cyber Bureau in the Prime Minister's Office. ${ }^{35}$ The resolutions further calls on the government to establish responsibility for dealing with the cyber field, albeit broadly. $^{36}$ Addendum $\mathrm{B}$ to the Resolution offers a model description of responsibilities, incorporating a Head Bureau position, ${ }^{37}$ Steering Committee, ${ }^{38}$ and related administrative working procedures. ${ }^{39}$ The third decision set forth by the Resolution was to advance defensive cyber capabilities in Israel, and to advance research and development in cyberspace and supercomputing. ${ }^{40}$ Numerous concrete policies were then detailed by the Resolution. These can be categorized as educational recommendations, policy compliance-related recommendations, and strategic recommendations.

To begin with, the Bureau's educational recommendations proactively identify and mitigate specific cyber security intimidations. The Bureau is consequently tasked with devising "national education plans," commonly aimed at "increasing public awareness" of cyber threats. ${ }^{42}$ Similar to the North Atlantic Treaty Organization (NATO), ${ }^{43}$ the United States Pentagon's Cyber Command (USCYBERCOM), ${ }^{44}$ Germany, ${ }^{45}$ United Kingdom, ${ }^{46}$ and Finland, ${ }^{47}$ the Israeli Cyber

\footnotetext{
E. Bambauer, "Privacy Versus Security," Journal of Criminal Law and Criminology vol. 103(3) (2013) (cyber security policy must focus on mitigating breaches rather than preventing them).

${ }^{33}$ Resolution No. 3611, supra note 28 ("[a]dvancing Israel's status as a center for the development of information technologies"). Thus two years after the establishment of the Israeli National Cyber Bureau, the Prime Minister, the Mayor of the southern metropolitan of city Beer-Sheva and the President of Ben Gurion University announced the establishment of a national cyber complex in Beer-Sheva, to be named CyberSpark, where INCB's command center also presides. Two giant international companies-Lockheed Martin and IBM have said to join Deutsche Telekom and EMC in setting up their research activities in the park. See Ben-Gurion University of the Negev, "Prime Minister Benjamin Netanyahu announces Creation of CyberSpark in Beer-Sheva" (January 27, 2014), http://in.bgu.ac.il/en/Pages/news/CyberSpark.aspx.

${ }^{34} I d$. ("encouraging cooperation among academia, industry and the private sector, government ministries and special bodies").

${ }^{35} I d$. at section $1,2$.

${ }^{36}$ Id. at section $2,2$.

${ }^{37}$ Id. at Addendum B, "Regulating Responsibilities for Dealing with the Cyber Field."

${ }^{38} I d$. at section B.

${ }^{39} \mathrm{Id}$. at sections $\mathrm{C}-\mathrm{H}$.

${ }^{40} I d$. at section 3, 2. Two subsidiary decisions follow. The fourth is a budgetary decision has been made in section 4 , stating: "The budget to implement this Resolution will be determined by the Prime Minister in consultation with the Minister of Finance, and will be submitted to the government for approval." The fifth decision upheld in section 5, at 2, excludes archetypical "Special bodies" from the mandate of the Bureau. Section D in the Definition part defines these as follows: "Special Bodies"- the Israel Defense Forces, the Israeli Police, Israel Security Agency ("Shabak"), the Institute for Intelligence and Special Operations ("Mossad") and the defense establishment by means of the Head of Security of the Defense Establishment (DSDE)."

${ }^{41} I d$. at Recommendation 14.

${ }^{42} I d$. at Recommendation 12. Recommendation 14 similarly calls for the "formulation of and the wise use of cyberspace."

${ }^{43}$ The Security \& Defense Agenda (SDA) report, supra note 23, at 71.

${ }^{44} I d$. at 83 .
} 
Bureau is said to coordinate national and international exercises, ${ }^{48}$ as well as cooperation with parallel bodies abroad. ${ }^{49}$

Secondly, the Resolution sets forth numerous recommendations over policy compliance. These recommendations essentially proffer tailored low-latency policy checkpoints. The Bureau henceforth is set to determine a yearly "national threat of reference," publish comparable ongoing "warnings" and "preventive practices.",

A national cyber situation room was put in charge of the bureau's early warning apparatus. The facility conducts ongoing national assessment among various essential civil and security and defense organizations while constituting a first national defensive layer for the entire country's administration. The national cyber situation room directly reports to INCB's central command. One telling occasion illustrates the cyber situation room's contribution. During Operation Pillar of Defense launched by Israel on November 14, 2012 against the Hamas-governed Gaza Strip, a massive overseas cyber attack was carried out against Israel. It included targeted distributed denial of services, the defacement of Israeli websites, and the publication of citizens' data. ${ }^{53}$ As INCB later announced, during the attack no publication of leaked data on any notable or potentially highly damaging scale occurred, largely because of the newly initiated cyber situation room. $^{54}$

The National Cyber Bureau is said to further advance coordination and cooperation between governmental bodies, the defense community, academia, industrial bodies, business, and other bodies relevant to the cyber field. ${ }^{55}$ One pivotal illustration demonstrates the organizational constraints with which the INCB operates in the midst of competing stakeholders. The body in Israel responsible for preventing cyber attacks on national infrastructure is the Shin Bet security service. The service also oversees the Israel National Information Security Authority (NISA). ${ }^{56}$ NISA is in charge of supervising and warning local governmental and private stakeholders with respect to defending against cyber terror and information security. NISA is further in charge of identifying and preventing cyber attacks over nationally labeled sensitive infrastructure, such as the Bank of Israel, the Israel Securities Authority, the Israel Electric Corporation, Israel Railways, and the Mekorot water company. ${ }^{57}$ INCB is not directly responsible for NISA's

\footnotetext{
${ }^{45} I d$. at 64 .

${ }^{46} I d$. at 80.

${ }^{4} \mathrm{Id}$. at 61 .

${ }^{48}$ Id. at Recommendation 9. In particular, Recommendations 10 and 11 offers to assemble intelligence picture from all intelligence bodies and similarly reiterate a "national situation status" concerning cyber security, respectively.

${ }^{49} I d$. at Recommendation 15. Substantive international cooperation is still deemed questionable by INCB, as discussed infra, Part C.2. See also Interview with Mr. Tal Goldstein from the Israeli National Cyber Bureau, supra note 3 .

${ }^{50} I d$. at Recommendation 5.

${ }^{51} \mathrm{Id}$. at Recommendation 13.

${ }^{52} \mathrm{Id}$. at Recommendation 13.

${ }^{53} \mathrm{Id}$.

${ }^{54} \mathrm{Id}$.

${ }^{55} \mathrm{Id}$. at Recommendation 16.

56 See Haaretz, Anshel Pfeffer and Gili Cohen, Who protects the Israeli civilian home front from cyber attack? (5.2.2013).

${ }^{57} \mathrm{Id}$.
} 
preventive activity but instead presumably is able to coordinate its activity vis-a-vis the Prime Minister at large. Surely, other cooperative initiative bear a more independent stand by INCB.

INCB conveniently categorizes its projects in three ways. These are (1) the development of national cyber security infrastructure, (2) the development of human capital and, finally, (3) the development of cyber defense (per the above mentioned national cyber security situation room). INCB has thus far initiated three national security-related projects with Israel's Office of the Chief Scientist (OCS), the Israeli Ministry of Defense, and Israeli academics. All involve a multistakeholder apparatus, albeit largely local and national. The first of the three is INCB's cooperation with Israel's Office of the Chief Scientist (OCS) in the Ministry of Economy. In a project called KIDMA (per the initials of the phrase "the promotion of cyber security $R \& D$ " in Hebrew), the Chief Scientist has adopted preferential policy for INCB's R\&D projects. In compliance with INCB's commitment to the promotion of cyber security R\&D, the KIDMA is officially aimed at promoting entrepreneurship within this field, while preserving and increasing Israel's competitive edge in cyber security world markets. ${ }^{58}$ In a program that started in 2013 , the Chief Scientist endowed the program with 80 million new Israeli shekels (approximately 22 million US dollars) for 2013-2014. ${ }^{59}$ A second national cyber security infrastructure project initiated by INCB was a 2012-2013 two-year collaboration with the local national security apparatus. Together with the Israeli Ministry of Defense's Research Authority, Development of Ammunition, and Technological Infrastructure (MAFAT), these two institutions have allocated a sum of 10 million Israeli new shekels (approximately USD 3.5 million) to a project called MASAD (per the initials of the term "Dual Cyber $R \& D$ " in Hebrew). ${ }^{60}$ This civil security project approaches cyber security challenges in two ways. It similarly was endowed with 32 million new Israeli shekels (approximately USD 10 million) for the years 2012-2014 and is specifically aimed at fostering academic research in the field.

A third national cyber security infrastructure project was initiated with university-based cyber security research centers. INCB has to date partnered two Israeli universities to establish two university research centers. These are the Ben-Gurion University of the Negev, with a research emphasis on technology and applicative sciences, and the Tel-Aviv University, with a broader interdisciplinary emphasis including political sciences and law. ${ }^{61}$

\footnotetext{
${ }^{58}$ Israel National Cyber Bureau publications, the Inauguration of the KIDMA-Promotion of R\&D in Cyber Security program (November 12, 2013) (in Hebrew), http://www.pmo.gov.il/MediaCenter/Spokesman/Pages/spokeKidma131112.aspx. See also Israel's Office of the Chief Scientist (OCS), Newsletter 02-2012 (21 Nov. 2012) (in Hebrew), http://www.moital.gov.il/NR/rdonlyres/89646959-5455-4A5A-99FD-C4B07D07E8E5/0/syber122012_3.pdf. The program includes upgraded funding for cyber security startups operating in technological incubators, a higher finance percentile in related venture capital funds, a fastened application examination process, etc. 
The underlying dual proposition continuously upheld by INCB has been that not only is academic research lagging behind the industry; but that this lag is in fact cross disciplinary, including non-technological fields and particularly social sciences and law. ${ }^{62}$ Accordingly, in May 2014 INCB published a novel grant program with the Israeli Ministry of Science as a part of its broad and interdisciplinary appeal which approaches not only scientists and engineers, but also political scientists and lawyers.

INCB has further developed a detailed program for promoting the development of human capital. INCB initiated cyber security advanced studies programs in numerous leading technological high schools and post-graduate academic programs. One such notable endeavor focuses on high schools from the country's socio-economically weaker regions. This project, entitled "Magshimim Leumit" ("Nationally Achieving" in Hebrew) is cooperating with the Israel's Ministry of Education in 2013-2016 to carry out a three year program focusing on educating and developing professional skills among outstanding high school students 16 to 18 years old. The program was founded under the assumption that cyber security is yet another policy avenue for the promotion of qualitative human capital within broader distributive justice dialectics. ${ }^{63}$

The Bureau regularly advises the Prime Minister, the government, and its committees regarding cyberspace. ${ }^{64}$ It is further said to consolidate the government's administrative cyberspace aspects $^{65}$ and to advance legislation and regulation in the cyber field. ${ }^{66}$ As of 2012, the INCB declared that the first ongoing regulatory stage would incorporate four types of regulation and accompanying objectives. These were the promotion of cyber security for organizations, the industrial and civil sectors, market regulation, and, lastly, cyber security regulation through standard setting. ${ }^{67}$ On this front, Israel's INCB has declared its plans to lead a process to establish recommendations for the government. ${ }^{68}$ As of July 2012, such a process was initiated by opening regular consultations with multistakeholder experts. This process took until October 2012 and focused on the limited issue of cyber law needs. ${ }^{69}$

\footnotetext{
${ }^{62} I d$.

${ }^{63}$ The Israeli Prime Minister's Office, "Announcement about the inauguration of the 'Magshimim Leumit' project," December 21, 2012, http://www.pmo.gov.il/MediaCenter/Events/Pages/eventmagshimim311212.aspx (in Hebrew).

${ }^{64} \mathrm{Id}$. at Recommendation 1. Notwithstanding the Bureau's overarching mandate, in matters of foreign affairs and security, the advice provided to the government, to its committees and to the ministers, will be provided on behalf of the Bureau by means of the Israeli National Security Council. Recommendation 19 offers that the Bureau carry out any other role in the cyber field determined by the Prime Minister.

${ }^{65} I d$. at Recommendation 2. The Bureau will also offer supporting cross agency coordination thereof.

Recommendation 4 further adds that the Bureau will "inform all the relevant bodies, as needed, about the complementary cyberspace-related policy guidelines."

${ }^{66} I$ d. at Recommendation 17. Recommendation 18 adds that the Bureau will serve as a regulating body regulating body in fields related to cyber security.

${ }^{67}$ See INCB's Public Consultation with multi stakeholders in preparing cyber security regulation (in Hebrew), http://www.pmo.gov.il/sitecollectiondocuments/pmo/cyber.doc.

${ }^{68} I d$.

${ }^{69} \mathrm{Id}$. It included four stages. at a start, INCB collected and processed expert testimonies. Soon after a public advisory committee was established. Then a series of open consultations as well as particular consultations took place. Lastly, INCB generated a list of recommendation which were at first open for public commentary soon to pass as INCB final regulation recommendation for the Israeli government consideration. See also interview with Mr. Amit Ashkenazi from the Israeli National Cyber Bureau (18 September 2014) (on file with author) (adding that it is
} 
Lastly, and more pertinently, there are three archetypical strategic propositions focused on establishing a measurable regulatory framework. The first strategic proposition solicits recommendations to be made "to the Prime Minister and government regarding national cyber policy." "70 The Israeli government and the Israeli Prime Minister's office thus assert themselves as direct participants in a cyber security policy model initiative. The second and third thematic, albeit overly general, strategic recommendations are to "promote research and development in cyberspace and supercomputing" "71 and devise a "national concept" for coping with "emergency situations in cyberspace." 72 These three policies are the foundation for this brief's legal positive framework.

\section{The Positive Framework}

This section maps the cyber security themes that constitute national cyber security policies worldwide. It introduces and discusses these fields of law with the goal of identifying the main legal concerns any national cyber security policy might address. First, it discusses cyber security definitions, including the range of cyber threats, types of cyber security risks, and types of practices not designated as cyber security risks. Second, models of cooperation over cyber security are reviewed, including inter-governmental, public-private platform (PPP), and regional cooperation. Lastly, the brief considers specific fields of law for examination, including cyber security and international law, cyber attacks and international humanitarian law, cyber treaties and international treaty law, national responsibility for cyber attacks and state responsibility, cyber crimes and cyber security, cyber attacks and international human rights law, privacy and cyber security, cyber security and telecommunications law, and cyber security and contractual obligations.

\section{A. Cyber Security Definitions}

A cyber security policy model should adhere to three categories of definitions, as these are repeatedly present in such leading policies.

- Defining the range of cyber threats, ranging from deliberate attacks for military or political advantage, through the forms of cyber crime and cyber warfare, and cyber terror against civil and military objects.

- Defining types of cyber security risks, ranging from concealment (Trojan horse), infectious malware and malware for profit (vector, control, maintenance, and payload), Botnets, cybercrime business models (advertising, theft, support), and chokepoints (antimalware, registrars, payments, site takedown, and blacklisting).

\footnotetext{
clear that adapted legislation is needed yet the intention is not to opt for an overarching statute but modular and proportional set of statutory frameworks for separate cyber threats).

${ }^{70}$ Id. at Addendum A, section 2 (titled "Bureau Goals"), Recommendation 3 ("[t]o guide the relevant bodies regarding the policies decided upon by the government and/or the Prime Minister; to implement the policy and follow-up on the implementation.").

${ }^{71}$ Id. at Recommendation 6. Such emblematic "research and development" should be promoted by what remain undefined "professional bodies."

${ }^{72}$ Id. at Recommendation 8 . There remains yet a fourth trade policy-related recommendation that albeit seminal in the Israeli Cyber Bureau's mandate, is nevertheless limited to advancement of the local economy. Recommendation 7 thus flatly calls upon the Bureau to "work to encourage the cyber industry in Israel," Recommendation 7 . This important yet only loosely irrelevant to cyber security policy, will remain outside the scope this policy brief.
} 
- Defining types of practices not designated as cyber security risks, including joke software, hoaxes, scams, spam, and cookies.

\section{B. Models of Cooperation Over Cyber Security}

A cyber security policy model ought to map the possibilities and limitations regarding the creation of cooperative international arrangements, involving governments and civil society to reduce risks to cyber security. INCB to date still witnesses only a limited degree of international cooperation. Two pivotal reasons account for this. First, few countries practice cyber security policies. A small number of countries actually have standing traditions of cyber industries supported by policy-making mechanisms and cyber security administrative organs. Second, it is questionable to what extent international consensus could be achieved, against the backdrop of regional and even narrower bilateral alternatives ${ }^{73}$ Notwithstanding these regulatory constraints, numerous avenues for cooperation exist across countries.

\section{Inter-Governmental Cooperation}

\section{a. $\quad$ The European Union}

The EU Cyber Security Strategy "Protection of an open and free Internet and opportunities in the digital world" (February 2013) and associated draft directives guide the EU's approach to cyber security. The Proposal for a Directive of the European Parliament and of the Council concerning measures to ensure a high common level of network and information security across the Union (February 2013) set the framework at the European Union for cyber security. The 2013 EU Cyber Security Strategy is being gradually implemented by EU member states with the purpose of minimizing policy fragmentation among member states.

The EU's policy resonates within the 2009 EU Commission's issuance of a communication on Critical Information Infrastructure Protection (CIIP), titled "Protecting Europe from large scale cyber-attacks and disruptions: enhancing preparedness, security and resilience." ${ }^{, 74}$ Similar to the Israeli case, the EU Commission recently noted that the upcoming challenges for Europe arebroadly-fourfold. First, there are uneven and uncoordinated national approaches by EU member states. Second, there is a need for a new European governance model for critical information infrastructures. Third, there is limited European early warning and incident response capability. Last, there is the need for appropriate international cooperation. Collaboration with non-European national cyber security policies, such as the Israeli policies, would satisfy the European Commission's call to engage the global community to develop a set of principles reflecting European core values for the net's resilience.

Moreover, a cyber security policy model could reflect cooperation to the extent of the European Programme for Critical Infrastructure Protection set forth in Directive EU COM(2006) 786. This program obliges all member states to the European Economic Area (EEA) to adopt the components of the Program into their national statutes. ${ }^{75}$ In the Israeli case, Israel and the EU are

\footnotetext{
${ }_{74}^{73}$ See Interview with Mr. Tal Goldstein from the Israeli National Cyber Bureau, supra note 3.

${ }^{74}$ See EU Commission, Protecting Europe from large-scale cyber-attacks and disruptions: enhancing preparedness, security and resilience (2009), http://ec.europa.eu/information_society/policy/nis/docs/comm_ciip/comm_en.pdf.

${ }^{75}$ European Programme for Critical Infrastructure Protection set forth in a Directive EU COM $(2006) 786$, http://eurlex.europa.eu/LexUriServ/site/en/com/2006/com2006_0786en01.pdf.
} 
continually discussing EEA's integration based on a direct association agreement with Israel so a harmonized cyber security policy brief may be issued in a timely manner.

Lastly, the European Union Agency for Network and Information Security (ENISA) operates for EU institutions and member states. ENISA serves as the EU's coordinated response to cyber security issues of the European Union and offers yet another platform for inter-governmental cooperation over cyber security in the EU. ${ }^{76}$

\section{b. $\quad$ The United States}

A cyber security policy model could further borrow from the case of the 2009 Comprehensive National Cyber-security Initiative (CNCI) put forward by the United States government and the subsequent 2011 International Strategy for Cyberspace (White House, May 2011). ${ }^{77}$ The details of this policy will be discussed in Part C.

Equally, most elements of the United States' policy focus on the federal government's cyber security, instead of cyber security on the state level. In balance, however, relative to the Israeli case, the United States has still not firmly decided what the regulatory apparatus of the federal government should be regarding the protection of critical infrastructure owned and operated by the private sector. Be that as it may, the United States' designated policy priorities include (1) economics, (2) protecting its networks, (3) law enforcement, (4) military, (5) Internet governance, (6) international development, and (7) Internet freedom, as will be discussed in Part C.

\section{Regional Cooperation}

One example of a regional or inter-regional cyber security initiative is the Asia-Pacific's regional cooperation over cyber security in the National Computer Emergency Response Teams (CERTs) by APCERT. This initiative facilitates regional cooperation and coordination amongst CERTs and Computer Security Incident Response Teams (CSIRTs). ${ }^{78}$

Another regional initiative is the comparable Organization of American States' (OAS) portal aimed at augmenting cyber security and regional responses to cybercrime. ${ }^{79}$ This rather earlystage portal was created primarily to facilitate and streamline cooperation and information exchange among government experts from OAS member states.

\section{Public-Private Platform (PPP)}

The business sector has taken on technological standardization initiatives since the early days of cyber security. Technological standardization has been advanced to increase the security of products, services, and networks. One such important initiative came from the Internet Corporation for Assigned Names and Numbers (ICANN). Its successful effort to promote development and adoption of security extensions for the domain name system (DNS) illustrates

\footnotetext{
${ }^{76}$ For ENISA's policy, see European Network and Information Security Agency (ENISA), "National Cyber Security Strategies: Practical Guide on Development and Execution," (December 2012).

77 The White House, President Barack Obama, "The Comprehensive National Cybersecurity Initiative," http://www.whitehouse.gov/cybersecurity/comprehensive-national-cybersecurity-initiative.

${ }^{78}$ Council for Security Cooperation in the Asia Pacific (CSCAP), Memorandum No. 20, Ensuring A Safer Cyber Security Environment (May 2012).

${ }^{79}$ See Inter-American Cooperation Portal on Cyber-Crime, http://www.oas.org/juridico/english/cyber.htm.
} 
how a private sector-led initiative backed by government participation can significantly enhance the Internet's security. ${ }^{80}$

Another important example for governmental cooperation with commercial enterprises and educational institutions, albeit with a technical orientation, are Computer Emergency Response Teams (CERTs). They are intended to promote information sharing and coordination among government agencies and the private sector against cyber-attacks, and identify and correct cybervulnerabilities. ${ }^{81}$ The lessons from CERTs are still rather preliminary and necessitate further technical testing.

\section{Economics of Information Security Considerations}

Evaluation of how different stakeholders' incentives align should include efficiency considerations. ${ }^{82}$ Thus, cyber security policy may offer not only direct regulation, but also indirect regulation aimed at incentivizing efficient behavior by end-users. To name but a few suggestions, these range from optimal security enhancing incentives, such as tax subsidies for compatible standards, to incentivizing whistle blowing against hazardous Internet users or even corporate espionage.

\section{Administrative Responses to Cyber Crises}

These offer additional possibilities regarding the creation of a cyber security policy model. This is the motivation for the Israeli Bureau's call to define emergency cyber situations in Recommendation 8 to the INCB's recommendations, and the Bureau's call for definition for cyber warnings in Recommendation 13 to the INCB's recommendations. This issue is discussed in Part C.

\section{6. $\quad$ Linking International Cyber Security Policy to Domestic Law}

Attention should be given to information asymmetries and principle-agent constraints when applying international cyber security regulation to the national sphere. ${ }^{83}$ Such legal adaptations generally support the need to preserve separate national discretion, realistic and even lenient regulatory timetables, administrative safe harbors, and even restrained judicial discretion, while still evaluating local differentiated security risks and challenges.

\footnotetext{
${ }^{80}$ See European Network and Information Security Agency (ENISA), “Good Practices Guide for Deploying DNSSEC," http://www.enisa.europa.eu/act/res/technologies/tech/gpgdnssec.

${ }^{81}$ See the Forum of Incident Response and Security Teams, http://www.first.org. The European Government CERTs (EGC) Group (http://www.egc-group.org) has 11 member organizations.

${ }^{82}$ Tyler Moore, Introducing the Economics of Cybersecurity: Principles and Policy Options, National Research Council, Proceedings of a Workshop (2010).

${ }^{83}$ Daniel Jacob Hemel, Regulatory Consolidation and Cross-Border Coordination: Challenging the Conventional Wisdom, Yale Journal on Regulation vol. 28(1) (2011) (offering a regulatory paradox whereby in areas where a single regulatory agency enjoys consolidated control over a particular policy matter at the domestic level, that agency is less willing to restrict its policy-making discretion through an international agreement and vice-versa).
} 


\section{A Cross-Section Comparison}

Over thirty countries have thus far declared archetypical national cyber security strategies. ${ }^{84}$ With quite a bit of similarity, countries have conducted active debates on codes of conduct for cyberspace, application of international laws, Internet governance and other aspects of functions, roles, and circumstances of cyberspace. National policies thus deal with the risks surrounding cyberspace from such viewpoints as national security and economic growth. Such national state practice has ultimately turned the functions, roles, and circumstances of cyberspace into a common international issue. Designing a cyber security policy model should therefore be especially attuned to leading national cyber security policies. This part offers a cross-sectional comparison between five such countries: the United States, the United Kingdom, Canada, Japan, and the Netherlands.

\section{United States}

\begin{tabular}{|c|c|c|}
\hline \multirow{4}{*}{$\begin{array}{c}\text { Types of } \\
\text { Cooperation }\end{array}$} & $\begin{array}{l}\text { Inter-governmental } \\
\text { cooperation }\end{array}$ & $\begin{array}{l}\text { Insert cyberspace issues on the agenda at the Organization of } \\
\text { American States (OAS), the Association of Southeast Asian } \\
\text { Nations (ASEAN) Regional Forum (ARF), the Asia-Pacific } \\
\text { Economic Cooperation Organization (APEC), the Organization } \\
\text { for Cooperation and Security in Europe (OSCE), the African } \\
\text { Union (AU), the Organization for Economic Cooperation and } \\
\text { Development (OECD), the Group of Eight (G-8), the European } \\
\text { Union (EU), the United Nations (U.N.), and the Council of } \\
\text { Europe. }\end{array}$ \\
\hline & $\begin{array}{l}\text { Public-Private platform } \\
\text { (PPP) }\end{array}$ & $\begin{array}{l}\text { The public and private sectors must work together to develop, } \\
\text { maintain, and implement standards and support the development } \\
\text { of international standards and conformity assessment schemes } \\
\text { that prevent barriers to international trade and commerce. }\end{array}$ \\
\hline & Regional cooperation & $\begin{array}{l}\text { Support the expansion of cyber security to geographic regions } \\
\text { currently underrepresented in the dialogue-most notably Africa } \\
\text { and the Middle East- }- \text { to further our interest in building } \\
\text { worldwide capacity. }\end{array}$ \\
\hline & $\begin{array}{l}\text { Intra-governmental } \\
\text { cooperation }\end{array}$ & $\begin{array}{l}\text { 1) Agencies across the United States Government are } \\
\text { collaborating, together with the private sector, to protect } \\
\text { innovation from industrial espionage, to protect Federal, state, } \\
\text { and local gov't networks, to protect military operations from } \\
\text { degraded operating environments, and to secure critical } \\
\text { infrastructure against intrusions and attacks. }{ }^{88} \\
\text { 2) Build and enhance existing military alliances to confront } \\
\text { potential threats in cyberspace. }\end{array}$ \\
\hline
\end{tabular}

\footnotetext{
${ }^{84}$ See, e.g., European Union Agency for Network and Information Security (ENISA), National Cyber Security Strategies in the World, http://www.enisa.europa.eu/activities/Resilience-and-CIIP/national-cyber-securitystrategies-ncsss/national-cyber-security-strategies-in-the-world (last retrieved 14 July 2014).

${ }^{85} \mathrm{Id}$.

${ }^{86} I d$.

${ }^{87} \mathrm{Id}$. at 18 .

${ }^{88} \mathrm{Id}$. at 19.

${ }^{89} \mathrm{Id}$. at 20.
} 


\begin{tabular}{|c|c|}
\hline & $\begin{array}{l}\text { 3)Given the Internet's importance to the world's economy, it is } \\
\text { essential that this network of networks and its underlying } \\
\text { infrastructure, the DNS, remain stable and secure. }\end{array}$ \\
\hline $\begin{array}{c}\text { Balancing } \\
\text { cyber security } \\
\text { with civil } \\
\text { liberties }\end{array}$ & $\begin{array}{l}\text { 1) Preserve, enhance, and increase access to an open, global Internet is a clear policy priority. } \\
\text { 2) Support civil society actors in achieving reliable, secure, and safe platforms for freedoms of } \\
\text { expression and association. }{ }^{92} \text { The same protections must apply to Internet Service Providers and } \\
\text { other providers of connectivity, who too often fall victim to legal regimes of intermediary } \\
\text { liability that pass the role of censoring legitimate speech down to companies. }{ }^{93} \\
\text { 3) Encourage international cooperation for effective commercial data privacy protections.... } \\
\text { The United States has a robust record of enforcement of its privacy laws, as well as } \\
\text { encouraging multi-stakeholder policy development. }{ }^{94}\end{array}$ \\
\hline $\begin{array}{l}\text { Form of } \\
\text { regulation } \\
\text { (legislation, } \\
\text { courts, } \\
\text { markets, } \\
\text { norms, etc) \& } \\
\text { institutional } \\
\text { aspects }\end{array}$ & $\begin{array}{l}\text { 1) Sustaining a free-trade environment while promoting international standards and innovative } \\
\text { open markets to ensure that cyberspace continues to serve the needs of our economies. }{ }^{95} \\
\text { 2) Developing international, voluntary, consensus-based cybersecurity standards and deploying } \\
\text { products, processes, and services based upon such standards. }{ }^{96}\end{array}$ \\
\hline $\begin{array}{c}\text { Promote cyber } \\
\text { security in } \\
\text { international } \\
\text { law }\end{array}$ & \multirow{2}{*}{$\begin{array}{l}\text { 1) Participate fully in international cybercrime policy developed bilaterally and multilaterally } \\
\text { like the Budapest Convention. } \\
\text { 2) The United States will continue to encourage other countries to become parties to the } \\
\text { Convention and will help current non-parties use the Convention as a basis for their own laws. } \\
\text { 3) Protect intellectual property, including commercial trade secrets, from theft and industrial } \\
\text { espionage. }{ }^{98} \text { The persistent theft of intellectual property, whether by criminals, foreign firms, or } \\
\text { state actors working on their behalf, can erode competitive-ness in the global economy, and }^{\text {businesses' opportunities to innovate. }}{ }^{99}\end{array}$} \\
\hline $\begin{array}{c}\text { Promote } \\
\text { counter cyber } \\
\text { crime policy }\end{array}$ & \\
\hline $\begin{array}{c}\text { Ensure } \\
\text { ongoing risk } \\
\text { assessment }\end{array}$ & $\begin{array}{l}\text { 1) Ensure robust incident management, resiliency, and recovery capabilities for information } \\
\text { infrastructure. }{ }^{100} \\
\text { 2) The United States Government actively participates in watch, warning, and incident response } \\
\text { through exchanging information with trusted networks of international partners. }{ }^{101} \\
\text { 3) The United States will also work to engage international participation in cybersecurity } \\
\text { exercises, to elevate and strengthen established operating procedures with our partners. }{ }^{102}\end{array}$ \\
\hline
\end{tabular}

${ }^{90} I d$. at 22.

${ }^{91} I d$. at 21 .

${ }^{92} \mathrm{Id}$. at 23.

${ }^{93} I d$. at 24 .

${ }^{94} \mathrm{Id}$.

${ }^{95} \mathrm{Id}$. at 17 .

${ }^{96} I d$. at 18 .

${ }^{97} \mathrm{Id}$. at 20 .

${ }^{98} I d$. at 17 .

${ }^{99} \mathrm{Id}$. at 18 .

${ }^{100} \mathrm{Id}$. at 19 .

${ }^{101} \mathrm{Id}$.

${ }^{102} I d$. 


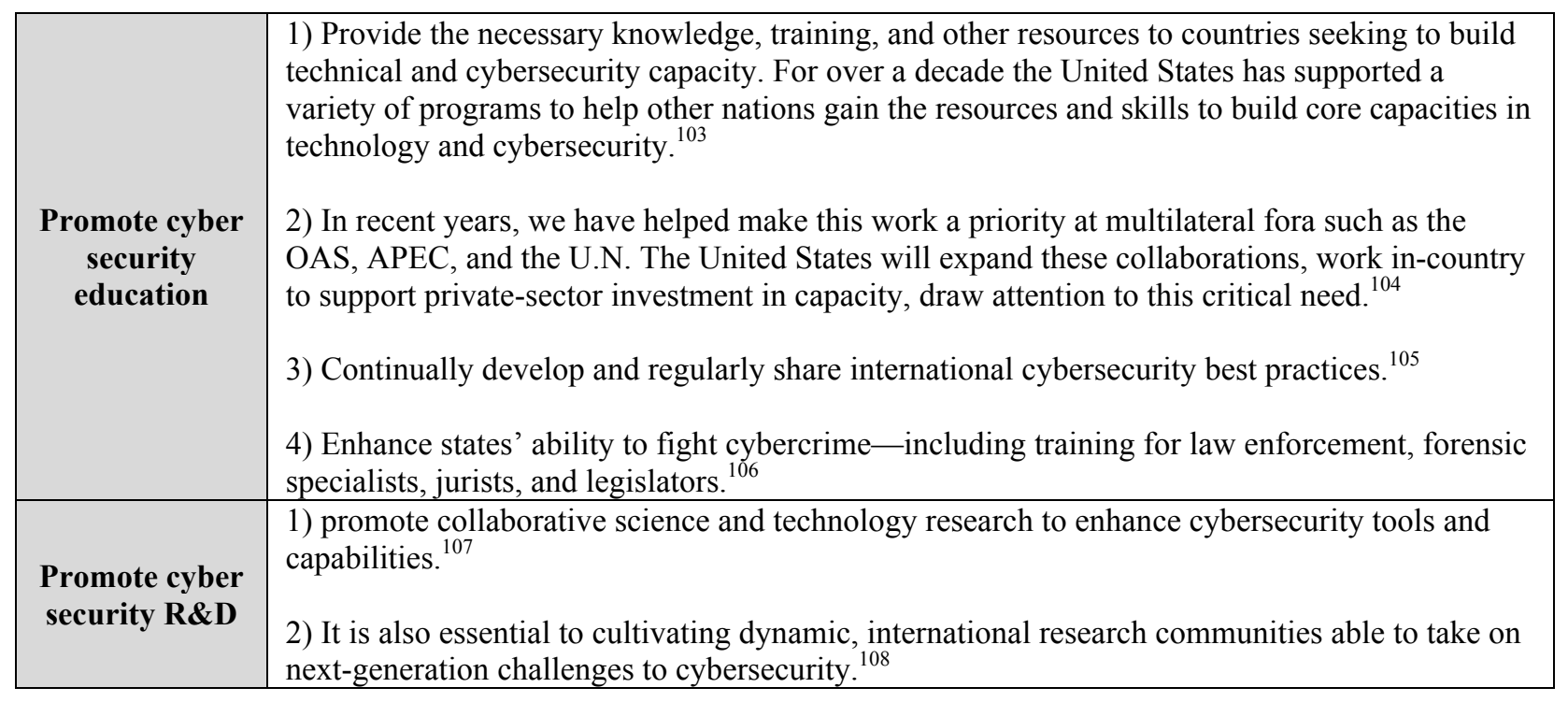

\section{United Kingdom (2009) ${ }^{109}$}

\begin{tabular}{|l|l|l|}
\hline $\begin{array}{c}\text { Types of } \\
\text { Cooperation }\end{array}$ & $\begin{array}{l}\text { 1) Threats are cross-border. We will seek partnership with other } \\
\text { countries that share our views. }{ }^{110} \\
\text { Inter- } \\
\text { 20vernmental } \\
\text { cooperation }\end{array}$ & $\begin{array}{l}\text { 2) Implement bilateral commitments set out in high-level } \\
\text { communiqués (agreed in } 201015 \text { and 2011) with the US, } \\
\text { Australia and France. }{ }^{111} \\
\text { 3) Develop new bilateral relationships on cyber with those } \\
\text { emerging powers that are active in cyberspace. }{ }^{112}\end{array}$ \\
& $\begin{array}{l}\text { Public-Private } \\
\text { platform (PPP) }\end{array}$ & $\begin{array}{l}\text { 1) require everybody, the private sector, individuals and } \\
\text { government to work together. }{ }^{113}\end{array}$ \\
& 2) The expertise and innovation required to keep pace with the \\
\hline
\end{tabular}

${ }^{103} I d$. at 22 .

${ }^{104} I d$.

${ }^{105} \mathrm{Id}$.

${ }^{106} I d$.

${ }^{107} I d$. at 23.

${ }^{108} I d$. at 15.

${ }^{109}$ The UK Cyber Security Strategy, Protecting and promoting the UK in a digital world (November 2011). Earlier, Great Britain published its 2009 policy initiative to promote growth via the Internet. See UK Cabinet Office, "Cyber Security Strategy of the United Kingdom: Safety, security and resilience in cyber space" (London: The Cabinet Office, CM 7642, June 2009), http://www.official-documents.gov.uk/document/cm76/7642/7642.pdf.

${ }^{110} \mathrm{Id}$. at 22.

${ }^{111} I d$. at 39 .

${ }^{112} \mathrm{Id}$.

${ }^{113} \mathrm{Id}$. at 22 .

${ }^{114} \mathrm{Id}$. 


\begin{tabular}{|c|c|c|}
\hline & & $\begin{array}{l}\text { continue to be safe and resilient. } \\
\text { 4) Seek agreement with ISPs on the support they might offer to } \\
\text { Internet users to help them identify, address, and protect } \\
\text { themselves from with malicious activity on their systems. }\end{array}$ \\
\hline & $\begin{array}{l}\text { Regional } \\
\text { cooperation }\end{array}$ & $\begin{array}{l}\text { 1) Work with allies to ensure implementation of NATO's cyber } \\
\text { defense policy (agreed in June 2011). } \\
\text { 2) Work closely with the European Commission and the } \\
\text { External Action Service to encourage greater coherence within } \\
\text { the EU on cyber issues. } \\
\text { 3) Encourage international and regional organizations to } \\
\text { support capacity building ... Work with the Commonwealth } \\
\text { (model legislation on cyber crime), the ITU (support training on } \\
\text { technical standards), the Council of Europe and with the } \\
\text { Organization for Security and Co-operation in Europe (OSCE) } \\
\text { to promote freedom of expression online. }\end{array}$ \\
\hline & $\begin{array}{c}\text { Intra- } \\
\text { governmental } \\
\text { cooperation }\end{array}$ & $\begin{array}{l}\text { 1) Create and build a dedicated and integrated civilian and } \\
\text { military capability within the MoD. Mainstreaming cyber } \\
\text { within the organization and setting up a Defense Cyber } \\
\text { Operations Group (DCOG). An interim DCOG will be in place } \\
\text { by April } 2012 \text { and will achieve full operational capability by } \\
\text { April } 2014 .^{120} \\
\text { 2) Support Olympic cyber security by joining up the relevant } \\
\text { departments and conducting exercises to ensure preparations } \\
\text { for cyber incidents are robust. }\end{array}$ \\
\hline $\begin{array}{l}\text { Balancing cyber } \\
\text { security with civil } \\
\text { liberties }\end{array}$ & \multicolumn{2}{|c|}{$\begin{array}{l}\text { 1) Support the open Internet, working with the Broadband Stakeholder Group to } \\
\text { develop industry-wide principles on traffic management and non-discrimination and } \\
\text { reviewing its transparency code of practice in early } 2012 .^{122} \\
\text { 2) Through the CONTEST strategy, increase our disruption of online radicalization and } \\
\text { recruitment, and safeguarding against cyber attack. } \\
\text { 3) Use multilateral and bilateral channels to discuss how to apply the framework of } \\
\text { international human rights law in cyberspace and new challenges in guaranteeing such } \\
\text { rights. } \\
\text { 4) Actively engage in the UN Group of Gov't Experts, which will reconvene in } 2012 \text {, } \\
\text { to ensure that a constructive report is made to the Secretary-General in } 2014 \text { in line }\end{array}$} \\
\hline
\end{tabular}

${ }^{115} \mathrm{Id}$. at 39 .

${ }^{116}$ Id. at 41.

${ }^{117} \mathrm{Id}$.

${ }^{118}$ Id. at 41.

${ }^{119} \mathrm{Id}$. at 40 .

${ }^{120} \mathrm{Id}$. at 39 .

${ }^{121}$ Id.

${ }^{122} \mathrm{Id}$. at 39

${ }^{123} \mathrm{Id}$.

${ }^{124} \mathrm{Id}$. at 40 . 


\begin{tabular}{|c|c|}
\hline & $\begin{array}{l}\text { with UN General Assembly Resolution 65/141 (driver of open societies, whilst } \\
\text { promoting stability and reliability). }{ }^{125}\end{array}$ \\
\hline $\begin{array}{l}\text { Form of } \\
\text { regulation } \\
\text { (legislation, } \\
\text { courts, markets, } \\
\text { norms, etc) \& } \\
\text { institutional } \\
\text { aspects }\end{array}$ & $\begin{array}{l}\text { 1) Create a new national cyber crime capability as part of the new National Crime } \\
\text { Agency by } 2013 .{ }^{126} \\
\text { 2) Working with domestic, European, global and commercial standards organizations } \\
\text { to stimulate the development of industry-led standards. }{ }^{127} \\
\text { 3) Support GetSafeOnline.org to become the single authoritative point of advice on } \\
\text { responding to cyber threats (for example, the recent publication of an Internet safety } \\
\text { guide). }{ }^{128} \\
\text { 4) Manage crucial skills and helping to develop a community of "ethical hackers" in } \\
\text { the UK to ensure that our networks are robustly protected. }\end{array}$ \\
\hline $\begin{array}{l}\text { Promote cyber } \\
\text { security in } \\
\text { international law }\end{array}$ & $\begin{array}{l}\text { The UK will continue to pursue the international development of norms of acceptable } \\
\text { behavior in cyberspace. according to principles proposed by the Foreign Secretary in } \\
\text { February } 2011 \text { and reiterated at the London Conference on Cyberspace (November } \\
2011):^{130} \\
\text { 1) Govn's should act proportionately in cyberspace and in accordance with national } \\
\text { and int'1. }{ }^{131} \\
\text { 2) maintain the ability in terms of skills, technology, confidence and opportunity- to } \\
\text { access cyberspace }{ }^{132} \\
\text { 3) [t]olerance and respect for diversity of language, culture and ideas }{ }^{133} \\
\text { 4) [o]penness to innovation and the free flow of ideas, information and expression }{ }^{134} \\
\text { 5) [r]espect individual rights of privacy and intellectual property }{ }^{135} \\
\text { 6) [c]ompetitive environment which ensures a fair return of investment }{ }^{136}\end{array}$ \\
\hline $\begin{array}{l}\text { Promote counter } \\
\text { cyber crime } \\
\text { policy }\end{array}$ & $\begin{array}{l}\text { 1) Promote greater levels of international cooperation and shared understanding on } \\
\text { cyber crime as part of the process begun by the London Conference on Cyberspace, in } \\
\text { addition to promoting the Council of Europe's Convention on Cybercrime (the } \\
\text { Budapest Convention) and building on the new EU Directive on attacks on information }\end{array}$ \\
\hline
\end{tabular}

${ }^{125} I d$. at 41 .

${ }^{126} \mathrm{Id}$. at 36 .

${ }^{127} \mathrm{Id}$. at 37 .

${ }^{128} \mathrm{Id}$. at 38 .

${ }^{129} \mathrm{Id}$. at 42 .

${ }^{130} \mathrm{Id}$. at 22 .

${ }^{131} \mathrm{Id}$.

${ }^{132} \mathrm{Id}$.

${ }^{133}$ Id.

${ }^{134} \mathrm{Id}$.

${ }^{135} \mathrm{Id}$.

${ }^{136} I d$. 


\begin{tabular}{|c|c|}
\hline & $\begin{array}{l}\text { systems. Contribute to the review of security provisions of the EU Data Protection } \\
\text { Directive and the proposed EU Strategy on Information Security. } \\
\text { 2) Encourage the courts in the UK to use existing powers to impose appropriate } \\
\text { online sanctions for online offences. } \\
\text { 3) Encourage the use of "cyber-specials" to bring in those with specialist skills to help } \\
\text { the police. }{ }^{139} \\
\text { 4) Significantly increase the law enforcement agency capability on cyber crime. }\end{array}$ \\
\hline $\begin{array}{l}\text { Ensure ongoing } \\
\text { risk assessment }\end{array}$ & $\begin{array}{l}\text { 1) [t]here can be no such thing as absolute security. We will therefore apply a risk- } \\
\text { based approach to prioritizing response. }{ }^{141} \\
\text { 2) Improve our ability to anticipate the technological, procedural and societal behavior } \\
\text { developments that affect our use of cyberspace. }{ }^{142} \\
\text { 3) Establish a scheme for certifying the competence of information assurance and } \\
\text { cyber security professionals by March } 2012 \text {, and a scheme for certifying specialist } \\
\text { training in } 2012 .{ }^{143}\end{array}$ \\
\hline $\begin{array}{l}\text { Promote cyber } \\
\text { security education }\end{array}$ & $\begin{array}{l}\text { 1) Raise awareness amongst businesses of the threat and actions that they can take to } \\
\text { protect themselves including working through strategically important sectors to raise } \\
\text { cyber security issues throughout their supply chains. }{ }^{144} \\
\text { 2) By March } 2012 \text {, conduct research on how to improve educational involvement with } \\
\text { cyber security significantly at all levels - including higher education and postgraduate } \\
\text { level. }{ }^{145}\end{array}$ \\
\hline $\begin{array}{l}\text { Promote cyber } \\
\text { security R\&D }\end{array}$ & $\begin{array}{l}\text { Enable the UK cyber security industry to thrive and expand, supporting it in accessing } \\
\text { overseas markets. }{ }^{146}\end{array}$ \\
\hline
\end{tabular}

${ }^{137} I d$. at 36 .

${ }^{138} I d$.

${ }^{139} \mathrm{Id}$.

${ }^{140} I d$.

${ }^{141} I d$. at 22.

${ }^{142} I d$. at 42 .

${ }^{143} \mathrm{Id}$.

${ }^{144} I d$. at 38 .

${ }^{145} \mathrm{Id}$. at 42 .

${ }^{146}$ The UK Cyber Security Strategy. Id. at 38 . 


\section{Canada ${ }^{147}$}

\begin{tabular}{|c|c|c|}
\hline \multirow{4}{*}{$\begin{array}{c}\text { Types of } \\
\text { Cooperation }\end{array}$} & $\begin{array}{l}\text { Inter- } \\
\text { governmental } \\
\text { cooperation }\end{array}$ & $\begin{array}{l}\text { 1) Builds upon our close working relationships with our allies } \\
\text { with special emphasis on Canada's closest security and } \\
\text { intelligence partners, the United States, the United Kingdom } \\
\text { and Australia. }{ }^{148} \\
\text { 2) To the extent possible, Canada will support efforts to build } \\
\text { the cyber security capacity of less developed states and foreign } \\
\text { partners. }{ }^{149}\end{array}$ \\
\hline & $\begin{array}{l}\text { Public-Private } \\
\text { platform (PPP) }\end{array}$ & $\begin{array}{l}\text { Emphasizes partnerships with Canadians, provinces, territories, } \\
\text { Business, NGOs and academia. }{ }^{150}\end{array}$ \\
\hline & $\begin{array}{l}\text { Regional } \\
\text { cooperation }\end{array}$ & $\begin{array}{l}\text { Canada will also build on its existing engagement in cyber } \\
\text { security discussions at key international fora, such as the United } \\
\text { Nations, NATO and the Group of Eight. }{ }^{151}\end{array}$ \\
\hline & $\begin{array}{l}\text { Intra- } \\
\text { governmental } \\
\text { cooperation }\end{array}$ & $\begin{array}{l}\text { 1) Partnering to secure vital cyber systems outside the federal } \\
\text { Government. }\end{array}$ \\
\hline $\begin{array}{l}\text { Balancing cyber } \\
\text { security with civil } \\
\text { liberties }\end{array}$ & \multicolumn{2}{|c|}{ Reflects Canadian values such as the rule of law, accountability and privacy. ${ }^{153}$} \\
\hline $\begin{array}{l}\text { Form of } \\
\text { regulation } \\
\text { (legislation, } \\
\text { courts, markets, } \\
\text { norms, etc) \& } \\
\text { institutional } \\
\text { aspects }\end{array}$ & \multicolumn{2}{|c|}{ Allows continual improvements to be made to meet emerging threats. ${ }^{154}$} \\
\hline \multicolumn{3}{|l|}{$\begin{array}{l}\text { Promote cyber } \\
\text { security in } \\
\text { international law }\end{array}$} \\
\hline $\begin{array}{l}\text { Promote counter } \\
\text { cyber crime }\end{array}$ & \multicolumn{2}{|c|}{$\begin{array}{l}\text { 1) The Government will strengthen the ability of law enforcement agencies to combat } \\
\text { cybercrime. }\end{array}$} \\
\hline
\end{tabular}

${ }^{147}$ See Government of Canada, Canada's Cyber Security Strategy for a Stronger and More Prosperous Canada, (2010), http://www.publicsafety.gc.ca/cnt/rsrcs/pblctns/cbr-scrt-strtgy/cbr-scrt-strtgy-eng.pdf.

${ }^{148} I d$. at 8 ("Three of our closest security and intelligence partners, the United States, the United Kingdom and Australia, recently released their own plans to secure cyberspace. Many of the guiding principles and operational priorities set out in those reports resemble our own.").

${ }^{149} I d$. at 9.

${ }^{150} I d$. at 9 ("Responsibility for digital security in the Netherlands lies with many parties. There is still insufficient cohesion between policy initiatives, public information, and operational cooperation. The Government therefore considers it essential to foster a collaborative approach between the public sector, the private sector, and knowledge institutions.").

${ }^{151} I d$. (Canada is "one of the non-European states that have signed the Council of Europe's Convention on Cybercrime").

${ }^{152} I d$.

${ }^{153} \mathrm{Id}$.

${ }^{154} \mathrm{Id}$. at 8 .

${ }^{155} \mathrm{Id}$. at 9. 


\begin{tabular}{|c|l|}
\hline policy & $\begin{array}{l}\text { 2) The Royal Canadian Mounted Police will investigate, as per the Royal Canadian } \\
\text { Mounted Police Act, suspected domestic and international criminal acts against } \\
\text { Canadian networks and critical information infrastructure. }\end{array}$ \\
& $\begin{array}{l}\text { 3) The Department of National Defense and the Canadian Forces will strengthen their } \\
\text { capacity to defend their own networks, will work with other Government } \\
\text { departments. }\end{array}$ \\
\hline $\begin{array}{c}\text { Ens } \\
\text { risk assessment }\end{array}$ & $\begin{array}{l}\text { 1) Within Public Safety Canada, the Canadian Cyber Incident Response Centre will } \\
\text { continue to be the focal point for monitoring and providing advice on mitigating cyber } \\
\text { threats. }\end{array}$ \\
2) The Canadian Cyber Incident Response Centre will direct the national response to \\
any cyber security incident.
\end{tabular}

\section{$\operatorname{Japan}^{162}$}

\begin{tabular}{|c|c|l|}
\hline $\begin{array}{c}\text { Types of } \\
\text { Cooperation }\end{array}$ & $\begin{array}{c}\text { Inter- } \\
\text { governmental } \\
\text { cooperation }\end{array}$ & $\begin{array}{l}\text { Cooperation with the United States, in which Japan is in an } \\
\text { alliance based on the Japan-U.S. Security Arrangements, is } \\
\text { vital. }^{163}\end{array}$ \\
\cline { 2 - 3 } & $\begin{array}{l}\text { Public-Private } \\
\text { platform (PPP) }\end{array}$ & $\begin{array}{l}\text { 1) the multi-stakeholders in cyberspace need to fulfill each } \\
\text { responsibilities corresponding to their respective roles in the } \\
\text { soeiety while muttully eopperating and assisting witheach } \\
\text { other including international cooperation and cooperation } \\
\text { between the public and private sectors. }\end{array}$ \\
\hline
\end{tabular}

${ }^{156}$ Id. ("The Canadian Security Intelligence Service will analyze and investigate domestic and international threats to the security of Canada."), at 10.

${ }^{157} I d$. at 10 .

${ }^{158} I d$. at 10

${ }^{159} \mathrm{Id}$.

${ }^{160} \mathrm{Id}$.

${ }^{161} I d$. at 13.

${ }^{162}$ See Japanese Information Security Policy Council, Information Security Strategy for protecting the nation (2013). Earlier the Japanese Information Security Policy Council released the Information Security Strategy for Protecting the Nation, (May 11, 2010), at: http://www.nisc.go.jp/eng/pdf/New_Strategy_English.pdf.

${ }^{163} \mathrm{Id}$. at 50 .

${ }^{164} I d$. at 22 . 


\begin{tabular}{|c|c|c|}
\hline & & $\begin{array}{l}\text { 2) [i]t is expected that private companies, educational } \\
\text { institutions and research institutions will work together in } \\
\text { industry-government-academia collaboration. }{ }^{165}\end{array}$ \\
\hline & $\begin{array}{l}\text { Regional } \\
\text { cooperation }\end{array}$ & $\begin{array}{l}\text { [t]he country will actively participate in multi country } \\
\text { discussions and meetings including regional frameworks such } \\
\text { as the ASEAN Regional Forum (ARF) Asia-Pacific forum and } \\
\text { other related committees in the United Nations. }{ }^{166}\end{array}$ \\
\hline & $\begin{array}{l}\text { Intra- } \\
\text { governmental } \\
\text { cooperation }\end{array}$ & $\begin{array}{l}\text { 1) [a]dvance threat analysis capabilities by promoting } \\
\text { information sharing and strengthen cooperation between } \\
\text { Computer Security Incident Response Team (CSIRT). }{ }^{167} \\
\text { 2) govn't must work to strengthen the functions of the NISC } \\
\text { (the "Cybersecu-rity Center" (tentative)) as a command post, } \\
\text { promote collaboration among relevant actors including between } \\
\text { ministries. }{ }^{168} \\
\text { 3) "Regarding Notation of Information Security Requireme-nts } \\
\text { in Procurement" was released to the various ministries, etc. on } \\
\text { January } 24,2012 \text { based on the results of the studies of the } \\
\text { "Subcommittee for Strengthening Public-Private Collaboration" } \\
\text { established in the Information Security Measure Promotion } \\
\text { Council (CISO Council) which is in turn established in the } \\
\text { Information Security Policy Meetings. }\end{array}$ \\
\hline $\begin{array}{l}\text { Balancing cyber } \\
\text { security with civil } \\
\text { liberties }\end{array}$ & \multicolumn{2}{|c|}{$\begin{array}{l}\text { 1) [i]t is important to multilaterally build and strengthen partnerships with other nations } \\
\text { and regions which share the same basic values including the basic policy, democracy, } \\
\text { respect for basic human rights, and the rule of law. For this reason, it is necessary to } \\
\text { carry out diplomacy which promotes a balanced approach to constructing a safe and } \\
\text { reliable cyberspace. }{ }^{170} \\
\text { 2) [c]yberspace has provided us a variety of positive benefits including innovation, } \\
\text { economic growth and solutions for social issues while still ensuring freedom of } \\
\text { expression and protection of privacy. }{ }^{171}\end{array}$} \\
\hline $\begin{array}{l}\text { Form of } \\
\text { regulation } \\
\text { (legislation, } \\
\text { courts, markets, }\end{array}$ & \multicolumn{2}{|c|}{$\begin{array}{l}\text { 1) Diverse entities such as the government, public, academic, industrial and private } \\
\text { sectors in Japan ... it becomes necessary for each entity to carry out their own } \\
\text { information security measures in an independent and proactive fashion as part of their } \\
\text { social responsibilities. }{ }^{172}\end{array}$} \\
\hline
\end{tabular}

165 Id. at 26 .

${ }^{166} \mathrm{Id}$. at 50

${ }^{167}$ Id. at 22 (CSIRT is a "system at businesses and government organizations for monitoring to check if any security issues exist with information systems and for carrying out investigations including cause analysis and extent of impact in the event an incident occurs").

${ }^{168} I d$. at 24.

${ }^{169} \mathrm{Id}$. at 32 .

${ }^{170} \mathrm{Id}$. at 49 .

${ }^{171} \mathrm{Id}$. at 20 .

${ }^{172} I d$. at 22. 


\begin{tabular}{|c|c|}
\hline $\begin{array}{l}\text { norms, etc) \& } \\
\text { institutional } \\
\text { aspects }\end{array}$ & $\begin{array}{l}\text { 2) [i]t is important that the whole of society participated in the "cyberspace hygiene" as } \\
\text { a preventative information security measure against unauthorized intrusions, malware } \\
\text { infections, vulnerabilities as factors for these incidents and other risks. }{ }^{173} \\
\text { 3) Japan has worked towards constructing a safe and reliable cyberspace in which free } \\
\text { flow of information is ensured by ensuring openness and interoperability of cyberspace } \\
\text { without excessively administering or regulating it. }{ }^{174} \\
\text { 4) The government must strengthen the basic functions of the nation related to } \\
\text { cyberspace. }{ }^{175} \\
\text { 5) [i]t is important for cyberspace-related operators to create a market through } \\
\text { development of advanced technologies and products, cultivation of human resources } \\
\text { with high abilities and the use and application of these resources for information } \\
\text { security measures in order to strengthen the international competitiveness of Japan's } \\
\text { "cybersecurity industry."176 }\end{array}$ \\
\hline $\begin{array}{l}\text { Promote cyber } \\
\text { security in } \\
\text { international law }\end{array}$ & $\begin{array}{l}\text { For the application of international laws to acts using cyberspace, it is important that } \\
\text { existing international laws continue to be applied to acts using cyberspace in terms of } \\
\text { maintaining a degree of order in cyberspace, and the deliberation will continue on how } \\
\text { to apply specific international laws such as the Charter of the United Nations and the } \\
\text { International Humanitarian Law to conducts in cyberspace. }{ }^{177}\end{array}$ \\
\hline $\begin{array}{l}\text { Promote counter } \\
\text { cyber crime } \\
\text { policy }\end{array}$ & $\begin{array}{l}\text { 1) System preparation will be carried out through expansion of organizations such as } \\
\text { the Cyber Attack Analysis Center, the Cyber Attack Special Investigations Unit and } \\
\text { the Unauthorized Program Analysis Center, information collection and analysis } \\
\text { equipment will be enhanced and strengthened and preparation of equipment including } \\
\text { the advancement of Internet monitoring systems. }{ }^{178} \\
\text { 2) The Japanese National Cyber-Forensics and Training Alliance (NCFTA) will take } \\
\text { measures for sharing information through cooperation with the private sector, } \\
\text { including the "Council to Prevent Unauthorized Communications as a Cyber } \\
\text { Intelligence Measure. } \\
\text { 3) Japan has ratified the Convention on Cybercrime and will work to strengthen rapid } \\
\text { and effective mutual investigations and other cooperation between law enforcement } \\
\text { agencies. }\end{array}$ \\
\hline
\end{tabular}

${ }^{173} \mathrm{Id}$.

${ }^{174} I d$. at 20.

${ }^{175} \mathrm{Id}$. at 23 .

${ }^{176} I d$. at 28 .

${ }^{177} I d$. at $49-50$.

${ }^{178} I d$. at 40 .

${ }^{179} I d$. at 40 (NCFTA is "a non-profit organization established in the United States and made up of members from the FBI, private sector businesses and academic institutions.").

${ }^{180}$ Id. at 52. The Convention on Cybercrime has been ratified in the Japanese Diet in April of 2004, coming into effect on November 1, 2012 through the enactment of the "Law for Partial Revision of the Penal Code, etc. to Respond to Increase in International and Organized Crimes and Advancement of Information Processing." 


\begin{tabular}{|c|c|}
\hline $\begin{array}{l}\text { Ensure ongoing } \\
\text { risk assessment }\end{array}$ & $\begin{array}{l}\text { 1) The Japanese Government Security Operation Coordination team (GSOC) was } \\
\text { formed in order to strengthen government institutions capability to deal with } \\
\text { emergencies related to information security issues such as external cyber attacks and } \\
\text { put into operation in April of } 2008 .{ }^{1{ }^{18}} \\
\text { 2) The collaboration among the GSOC, the CYMAT } 84 \text { and the CSIRT of each } \\
\text { government institution at the time of incident occurrence shall be strengthened in order } \\
\text { for immediate sharing of incident information and a full readiness system by the } \\
\text { government together. In addition, in anticipation of large-scale cyber attacks } 85 \text { and } \\
\text { these possibilities countermeasures for the occurrence of incidents. } \\
\text { 3) The Japanese government established the Capability for Engineering of Protection, } \\
\text { Technical Operation, Analysis and Response (CEPTOAR) system for sharing and } \\
\text { analyzing information in the } 10 \text { critical infrastructure fields in Japan. }{ }^{183}\end{array}$ \\
\hline $\begin{array}{l}\text { Promote cyber } \\
\text { security education }\end{array}$ & $\begin{array}{l}\text { 1) [i]t is important that in addition to the understanding that small and medium-sized } \\
\text { enterprises "are responsible for protecting themselves" general users must also make } \\
\text { efforts to implement measures based on an awareness of "not bothering others." } \\
\text { 2) [i]t is necessary to plan awareness raising activities starting from the elementary and } \\
\text { middle school education stages, and implement participatory awareness raising projects } \\
\text { such as motto and poster contests. }\end{array}$ \\
\hline $\begin{array}{l}\text { Promote cyber } \\
\text { security } R \& D\end{array}$ & $\begin{array}{l}{[\mathrm{r}] \text { esearch and development and practical testing of technologies aimed at improving }} \\
\text { the cyber attack detection and advanced analysis functions at research institutions and } \\
\text { relevant organizations shall be accelerated. }{ }^{186}\end{array}$ \\
\hline
\end{tabular}

\footnotetext{
${ }^{181} I d$. at 32 .

182 Japanese Information Security Policy Council, Information Security Strategy for protecting the nation (2013), at 33. Cyber incident Mobile Assistant Team (Information security emergency support team) (CYMAT) was established in in June of 2012, and provides technical support and advice related to preventing spread of damages, recovery, cause investigation and recurrence prevention in the event of cyber-attacks. ministries or other agencies under the National Information Security Center director who is the government CISO.

183 Japanese Information Security Policy Council, Information Security Strategy for protecting the nation (2013), at 34.

${ }^{184} I d$. at 27.

${ }^{185} \mathrm{Id}$. at 48 .

${ }^{186}$ Japanese Information Security Policy Council, Information Security Strategy for protecting the nation (2013), at 45.
} 


\section{The Netherlands ${ }^{187}$}

\begin{tabular}{|c|c|c|}
\hline \multirow{4}{*}{$\begin{array}{c}\text { Types of } \\
\text { Cooperation }\end{array}$} & $\begin{array}{c}\text { Inter- } \\
\text { governmental } \\
\text { cooperation }\end{array}$ & $\begin{array}{l}\text { 1) Active international cooperation }{ }^{188} \text { Note-The International } \\
\text { Security Strategy is aimed at actions taken by the Netherlands } \\
\text { abroad and in cooperation with other countries to secure its } \\
\text { interests. }\end{array}$ \\
\hline & $\begin{array}{l}\text { Public-Private } \\
\text { platform (PPP) }\end{array}$ & $\begin{array}{l}\text { 1) Public-private partnerships } \\
\text { 2) Military and civil, public and private, national and } \\
\text { international actors have become more intertwined. }{ }^{191}\end{array}$ \\
\hline & $\begin{array}{l}\text { Regional } \\
\text { cooperation }\end{array}$ & $\begin{array}{l}\text { The Dutch NCSS2 is in line with the fundamental principles of } \\
\text { the EU Cyber Security Strategy. }\end{array}$ \\
\hline & $\begin{array}{c}\text { Intra- } \\
\text { governmental } \\
\text { cooperation }\end{array}$ & $\begin{array}{l}\text { 1) Division of responsibilities between ministries }{ }^{193} \\
\text { 2) Risk analyses, security requirements and information sharing } \\
\text { within critical infrastructure sectors }{ }^{194} \\
\text { 3) Enhancing civil-military cooperation }\end{array}$ \\
\hline $\begin{array}{l}\text { Balancing cyber } \\
\text { security with civil } \\
\text { liberties }\end{array}$ & \multicolumn{2}{|c|}{$\begin{array}{l}\text { 1) The Netherlands builds coalitions for freedom, security and peace in the digital } \\
\text { domain. }\end{array}$} \\
\hline
\end{tabular}

${ }^{187}$ See National Cyber Security, "Strategy 2: From awareness to capability" (2013); see also Ministry of Science and Justice, The National Cyber Security Strategy (NCSS), "The Ministry of Security and Justice, the Netherlands" (2011).

188 The National Cyber Security Strategy (NCSS), The Ministry of Security and Justice, the Netherlands (2011). See id. at 6 ("The Netherlands supports and actively contributes to efforts such as the EU's Digital Agenda for Europe and Internal Security Strategy, NATO's development of cyber defense policy as part of its new strategic vision, the Internet Governance Forum, and other partnerships.").

${ }^{189} \mathrm{Id}$. at 9.

190 The National Cyber Security Strategy (NCSS), "The Ministry of Security and Justice, the Netherlands" (2011), at 5 ("Every party concerned must gain value from participation in joint initiatives - an outcome that will be facilitated by an effective cooperation model with clearly defined tasks, responsibilities, powers, and guarantees.").

191 See National Cyber Security, Strategy 2: From awareness to capability (2013); id. at 14.

192 Id. at 9.

193 The National Cyber Security Strategy (NCSS), The Ministry of Security and Justice, the Netherlands (2011), Id. at 6 ("The Minister of Security and Justice is, in accordance with the National Security Strategy, responsible for coherence and cooperation on cyber security. At the same time, each party in the cyber security system has its own tasks and responsibilities.").

${ }^{194}$ See National Cyber Security, Strategy 2: From awareness to capability (2013); id. at 9 ("the government, working with vital parties, identifies critical ICT-dependent systems, services and processes.").

195 Id. at 9.

196 The National Cyber Security Strategy (NCSS), The Ministry of Security and Justice, the Netherlands (2011), Id. at 6 ("[i]t aims to protect our society's core values, such as privacy, respect for others, and fundamental rights such as freedom of expression and information gathering. We still need a balance between our desire for public and national security and for protection of our fundamental rights."). 


\begin{tabular}{|c|l|}
\hline $\begin{array}{c}\text { Form of } \\
\text { regulation } \\
\text { (legislation, } \\
\text { courts, markets, } \\
\text { norms, etc) \& } \\
\text { institutional } \\
\text { aspects }\end{array}$ & 1) Strengthening the National Cyber Security Centre ${ }^{197}$ \\
Computer Emergency Response Team (CERT). ${ }^{198}$ & 4) Self-regulation if possible, legislation if necessary. ${ }^{200}$ \\
\hline $\begin{array}{c}\text { Promote cyber } \\
\text { security in } \\
\text { international law }\end{array}$ & $\begin{array}{l}\text { 1) develop a hub for expertise on international law and cyber security "Cyber } \\
\text { diplomacy"). }{ }^{201}\end{array}$ \\
\hline $\begin{array}{c}\text { 2) More active approach to cyber espionage. }{ }^{202} \\
\text { Promote counter } \\
\text { cyber crime } \\
\text { policy }\end{array}$ & $\begin{array}{l}\text { 1) International approach to cyber crime: updating and strengthening legislation } \\
\text { (including the Criminal "Cyber diplomacy"). }{ }^{203}\end{array}$ \\
\hline 2) More active approach to cyber espionage. ${ }^{204}$ \\
\hline $\begin{array}{c}\text { Ensure ongoing } \\
\text { risk assessment }\end{array}$ & $\begin{array}{l}\text { 2) Ensure appropriate and up-to-date threat and risk assessments. }{ }^{205} \\
\text { managers. With the Taskforce on Management, Information Security and Services, the } \\
\text { government pursues an active awareness policy to get the government's information } \\
\text { security at the desired level. }{ }^{206}\end{array}$ \\
\hline
\end{tabular}

${ }^{197} I d$. at 10 ("the NCSC assumes the role of expert authority, providing advice to private and public parties involved, both when asked and at its own initiative.").

${ }^{198} I d$. ("Finally, based on its own detection capability and its triage role in crises, the NCSC develops into Security Operations Centre (SOC) in addition to its role as a Computer Emergency Response Team (CERT).”).

${ }^{199}$ Id. ("Together with private sector partners, the government works to develop standards that can be used to protect and improve the security of ICT products and services.").

${ }^{200}$ The National Cyber Security Strategy (NCSS), The Ministry of Security and Justice, the Netherlands (2011), Id. at 6 ("The public and private sectors will achieve the ICT security they seek primarily through self-regulation. If self-regulation does not work, the Government will examine the scope for legislation.").

${ }^{201} I d$. ("The goal of the hub for expertise is to promote the peaceful use of the digital domain. To this end, the Netherlands combines knowledge from existing centers. The centre brings together international experts and policymakers, diplomats, military personnel and NGOs.").

${ }^{202}$ Id. at 9 ("To this end, the intelligence and security services have combined their cyber capabilities in the Joint Sigint Cyber Unit (JSCU)"). Both 2010 (cyber conflict) and 2012 (cyber espionage) cyber security scenarios were included in the NV Strategy. Id. at 14.

${ }^{203} I d$. ("The goal of the hub for expertise is to promote the peaceful use of the digital domain. To this end, the Netherlands combines knowledge from existing centers. The centre brings together international experts and policymakers, diplomats, military personnel and NGOs.”).

${ }^{204}$ Id. at 9 ("To this end, the intelligence and security services have combined their cyber capabilities in the Joint Sigint Cyber Unit (JSCU)"). Both 2010 (cyber conflict) and 2012 (cyber espionage) cyber security scenarios were included in the NV Strategy. Id. at 14.

${ }^{205}$ See National Cyber Security, Strategy 2: From awareness to capability (2013); id. at 8 .

${ }^{206} \mathrm{Id}$. at 14 ("This is not only an important precondition for the implementation of the government's plans concerning the concept of the digital government 2017, but also in view of the Government-Wide Implementation Agenda for eGovernment Services until 2015 (i-NUP), in which a basic infrastructure will be realized."). 


\begin{tabular}{|c|c|}
\hline $\begin{array}{l}\text { Promote cyber } \\
\text { security education }\end{array}$ & $\begin{array}{l}\text { 1) Promote research and education in cyber security }{ }^{207} \\
\text { 2) Taskforce on cyber security education }{ }^{208} \\
\text { 3) Individual responsibility }{ }^{209}\end{array}$ \\
\hline $\begin{array}{l}\text { Promote cyber } \\
\text { security R\&D }\end{array}$ & $\begin{array}{l}\text { 1) Promote research and education in cyber security } \\
\text { 2) Encouraging innovation in cyber security } \\
\text { 3) Feasibility study on separate vital network } \\
\text { 312 }\end{array}$ \\
\hline
\end{tabular}

\section{Best Practices and Conclusion}

Israel's establishment of the INCB cyber command and its upward national cyber policy have five facets. These are: 1) the implementation of a medium-run five-year plan to scale up the country as a world leader in cyber security; 2) the inclusion of investment in research and development (R\&D) based on interdisciplinary university research centers and backed by extensive governmental funding; 3) encouraging industry to develop new technologies; 4) the setting up of a super computer center; and 5) boosting academic studies in cybernetics.

The effectiveness of Israel's cyber policy is still unfolding, and several caveats also labeled as best practices for future national cyber security policy models apply. First, cyber security is still an evolving discipline where future cyber risks and threats are largely unknown. Any cyber security policy model should thus reflect this and include regulatory modularity and administrative flexibility. Furthermore, national cyber security policies often carry a reactive nature as they regularly emerge after equivalent cyber threats evolve, and Israel's experience is no different. As a result, taken from the organizational angle, cyber security policies do not replace administrative organizations because these organizations end up coordinating the policies. Israel's INCB serves as proof of this situation. INCB's rather modest thirty-employee core scarcely has the means to battle cyber threats directly. Instead, INCB coordinates the cyber battles of myriad local defenses and civil agencies and corporations. A third caveat calling for

\footnotetext{
207 Id. at 8.

${ }^{208}$ Id. ("To enlarge the pool of cyber security experts and enhance users' proficiency with cyber security, the business community and the government join forces to improve the quality and breadth of ICT education at all academic levels (primary, secondary and professional education)").

209 The National Cyber Security Strategy (NCSS), The Ministry of Security and Justice, the Netherlands (2011), Id. at 6 ("All users (individuals, businesses, institutions, and public bodies) should take appropriate measures to secure their own ICT systems and networks and to avoid security risks to others".).

${ }^{210}$ Id. at 8 .

${ }^{211}$ Id. National Cyber Security, Strategy 2: From awareness to capability (2013) ("coordination of supply and demand, which can be achieved by linking innovation initiatives to leading sector policy. In addition, the government, the business community and the world of academia will launch a cyber security innovation platform where start-ups, established companies, students and researchers can connect, inspire one another and attune research supply and demand.").

${ }^{212} I d$. at 9 ("An exploratory study is conducted to determine whether it is possible and useful, from both a technical and organizational perspective, to create a separate ICT network for public and private vital processes.”).
} 
certain restraint applies in the Israeli example. Accordingly, different than in most cyber-literate countries worldwide, Israel's INCB materialized in reaction to momentous national security threats unfamiliar or at least moderately undemanding in relation to most of its counterparts. The fact that the cyber attacks that Israel faced were national security threats, as opposed to the less dangerous cyber crime that many other nations face, led to a different kind of regulatory regime.

That said, this comparison of policies yields some observations on commonalities between the policies of the United States, the United Kingdom, Canada, Japan, the Netherlands and Israel:

Promoting cyber security $R \& D$ : In the experiences of the United States, Israel, and the United Kingdom, national commitment to research and development in cyber security was essential for two main reasons. Firstly, it cultivates dynamic international research communities able to take on next-generation challenges to cyber security. Secondly, it enables national cyber security industries to expand while supporting them in accessing overseas markets. In each country, such practices are adapted to the specific scientific educational frameworks and underlying national preferences.

Promote cyber security education: There seem to be three types of educational policies within the cyber security context. The first are educational programs that help nations gain the resources and skills to build core capacities in technology and cyber security. The promotion of cyber security education is meant to raise awareness among businesses of this threat and of the protective actions they may take. In recent years, the United States most noticeably helped make education around cyber security a priority at multilateral forums such as the OAS, APEC, and the UN. Cyber security-related education and training has another purpose within the related context of cyber crime. In this context cyber security, educational, and training programs are aimed at law enforcement officials, forensic specialists, jurists, and legislators. The third type of educational policy is intended to improve educational involvement at the higher education and postgraduate level, and construct a vibrant research community and related cyber security industries.

Ensuring ongoing risk assessment: All national cyber security policies reviewed have developed a detailed watch, warning, and incident response to cyber threats through exchanging information within trusted networks. Similarly, national policies systematically participate in national and international cyber security exercises to elevate and strengthen established security procedures. Lastly, national policies have established equivalent schemes for certifying the competence of information assurance and cyber security.

Promote counter cyber crime policy: Cyber crime policy has developed both multilaterally, such as through the Council of Europe's Convention on Cyber Crime (the Budapest Convention), and bilaterally. Given cyber crime's international character, it is important to encourage other countries to become parties to the Convention and help current non-parties use the Convention as a basis for their own laws. Within the European context, cyber crime policy further builds upon the new EU Directive on attacks on information systems. Equally, all reviewed countries have committed to increase their law enforcement capabilities to combat cybercrime. In balance, however, a cyber security policy model should carefully scale institutional preferences related to online law enforcement at large. Canada, to name one example, has delegated to the Royal Canadian Mounted Police domestic and international enforcement responsibilities. Yet the 
Canadian Security Intelligence Service, by the same token, is mandated to analyze and investigate domestic and international threats to the security of Canada.

Promote cyber security in international law: All countries reviewed share a unified commitment to the rule of law in cyberspace and to international law. The United Kingdom noticeably has explicitly adopted an additional international norms-based policy of tolerance and respect for diversity of language, culture, and ideas. The Netherlands added a commitment to upholding peace in cyber security enforcement. Among the individual rights mentioned are rights of privacy, freedom of speech, and intellectual property. National policies reviewed have not mentioned international humanitarian law or state responsibility policy preferences. For the application of international laws to cyberspace, it is important that existing international laws be adapted, although key binding treaties and international laws (and even mere state practice) are still missing.

Form of regulation (legislation, courts, markets, norms, etc) \& institutional aspects: The United States unlike other reviewed countries has elaborated the role of technological standards in regulating cyber security. It has consequently called for industry-government cooperation over an open, voluntary, and compatible standardization of the Internet's security. The US government further reiterates its understanding that such standard setting is not only commercially beneficial to the US economy, but should also be industry-led. There is thus a conceptual gap between the United States and Canada, the United Kingdom, and the Netherlands over this issue. The latter countries implicitly undermine the role of standards in regulating the Internet's security as they opt for either self-regulation, such as in the Netherlands, or state regulation backed by judicial review, in the case of the United Kingdom or Canada.

Balancing cyber security with civil liberties: National cyber security policies share a commitment to enhancing access to a secure, private, reliable, and safe Internet. The United States further offers to protect Internet service providers (ISPs) and other types of providers. The US national policy provides such protection while explaining that ISPs too often fall victim to legal regimes that pass off the role of censoring legitimate speech to such companies. Balancing cyber security with civil liberties is further promoted by international and regional partnerships with countries that share comparable basic values. Such values mentioned were commonly associated with freedom of speech and association, privacy, respect for basic human rights, and the rule of law.

Type of cooperation: Cyber security policies seem to be deeply intertwined with cooperation between countries internationally and regionally. Leading regional cooperative frameworks are NATO's cyber defense policy, ASEAN Regional Forum (ARF) Asia-Pacific forum, the Council of Europe, and the Organization for Security and Co-operation in Europe. Governments similarly collaborate with the private sector in public-private platform (PPP) initiatives in order to protect federal, state, and local governments, as cyber threats are said to be business-driven in part. The United States' cyber security policy further calls for enhancing civil-military cooperation.

In international cooperation, national cyber security policies deem cyber threats to be strongly associated with countries that share similar socio-political values and interests. The countries reviewed were Western democratic countries, or otherwise close security and intelligence partners. Examples include Canada's close intelligence partnerships with the United States, the United Kingdom, and Australia, and Japan's strategic alliance with the United States. 


\section{Appendix I: \\ A Bill of Rights for the Brazilian Internet ("Marco Civil") - A Multistakeholder Policymaking Case}




\title{
NoC Internet Governance Case Studies Series: A Bill of Rights for the Brazilian Internet ("Marco Civil") - A Multistakeholder Policymaking Case
}

\author{
Ronaldo Lemos, Fabro Steibel, Carlos Affonso de Souza, and Juliana Nolasco \\ Institute for Technology and Society at Rio de Janeiro State University
}

\begin{abstract}
Editorial Note: Context, Character, and Purpose of the Case Study
This case study is part of a globally coordinated, independent academic research pilot project by the Global Network of Interdisciplinary Internet \& Society Research Centers (NoC). Facilitated by the Berkman Center for Internet \& Society at Harvard University, this study examines existing multistakeholder governance groups with the goal of informing the future evolution of the Internet governance ecosystem. Building upon the NETmundial Principles and Roadmap, it contributes to current policy debates at the international level, including the Internet Governance Forum, the NETmundial Initiative, and other organizations and efforts.
\end{abstract}

Internet governance is an increasingly complex concept that operates at multiple levels and in different dimensions, making it necessary to have a better understanding of both how multistakeholder governance groups operate and how they best achieve their goals. With this need in mind, at a point where the future of Internet governance is being re-envisioned, colleagues from several NoC institutions around the world have written twelve case studies examining a geographically and topically diverse set of local, national, and international governance models, components, and mechanisms from within and outside of the sphere of Internet governance. Key findings from these cases are summarized in a synthesis paper, which aims to deepen our understanding of the formation, operation, and critical success factors of governance groups and even challenge conventional thinking.

The research, based on twelve case studies, suggests that there is no single best-fit model for multistakeholder governance groups that can be applied in all situations. Rather, it reveals a range of approaches, mechanisms, and tools available for both the formation and operation of such groups. The analysis demonstrates that the success of governance groups depends to a large degree on the careful selection, deployment, and management of suitable instruments from this "toolbox." As governance groups pass through different phases of operation, conveners and facilitators must remain alert to changes in circumstances that necessitate adjustments to the approaches, mechanisms, and tools that they deploy in order to address evolving challenges from inside and outside. This case study provides insights into how those instruments can be deployed and adjusted over time within such groups, and highlights how their interactions with important contextual factors may be successfully managed within given resource restraints.

The research effort is grounded in a diversity of global perspectives and collaborative research techniques. Adhering to objective and independent academic standards, it aspires to be useful, actionable, and timely for policymakers and stakeholders. More broadly, the Network of Centers seeks to contribute to a more generalized vision and longer-term strategy for academia regarding its roles in research, facilitation and convening, and education in and communication about the Internet age.

For additional information on the initiative, please contact Urs Gasser, Berkman Center for Internet \& Society, at ugasser@cyber.law.harvard.edu. 


\begin{abstract}
This case study explores the events that led to the passing of the "Marco Civil da Internet" legislation, which became law in Brazil in April 2014. The first of its kind anywhere in the world, Marco Civil is a landmark "Bill of Rights" for the Brazilian Internet users. The legislative is unique both due to its substance and the process by which it was created. From a substantive standpoint, Marco Civil applies to the Internet key principles such as freedom of expression, net neutrality, and due process. But perhaps more important than the substance is the way in which it was drafted and enacted. This case study explores the unique features of that drafting process. Marco Civil was conceptualized in 2007 and, over the course of many years, was drafted using an open multistakeholder process through which members of the public, government, global and local internet companies, civil society, and others engaged in negotiations over the legislation's text, largely mediated through online platforms. Although the law was ultimately adopted, the process was not without its challenges. For example, attracting early contributors and participants was difficult, as was debating contentious topics openly among the stakeholders, who frequently disagreed. After many delays, Marco Civil was eventually passed. This case study will explore the factors that enabled a complex piece of legislation to be drafted with input and consultation from a distributed and diverse collection of stakeholders.
\end{abstract}




\section{Table of Contents}

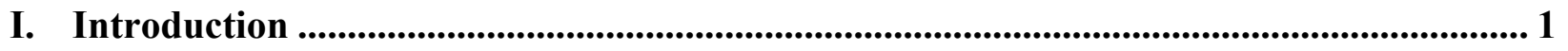

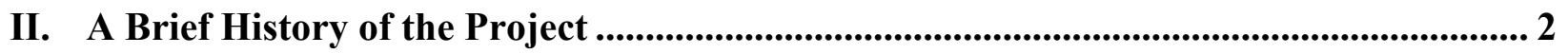

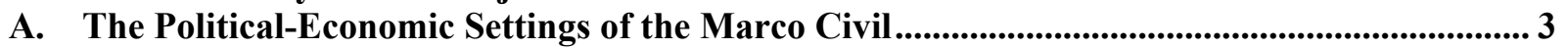

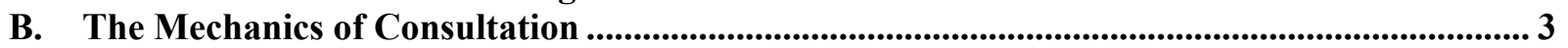

III. Successes of the Multistakeholder Process in Marco Civil .................................................. 4

IV. Challenges of Multistakeholderism in Marco Civil .......................................................... 7

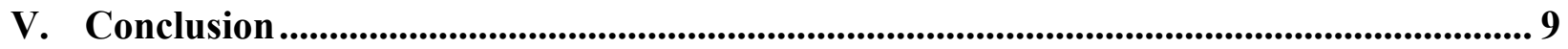




\section{Introduction}

The "Marco Civil da Internet" was the first piece of legislation worldwide to regulate the Internet from the perspective of fundamental human rights. The Marco Civil, passed in April 2014, establishes a comprehensive "Bill of Rights" for the Internet. Its passage follows both the Web's 25th anniversary and Sir Tim Berners-Lee's call for a "Magna Carta" for the Internet. Aside from its unique substance, the Marco Civil is also notable for its multistakeholder policy making process. The Marco Civil, in contrast to other draft bills intended to regulate Brazil's Internet, was not drafted in a purely top-down or a bottom-up process. Instead, the Marco Civil was drafted through a collaborative and unorganized effort that involved civil society, government (executive and legislative branches), academics, the technical community, and the business sector. As such, Marco Civil was the product of an open and collaborative effort involving an array of stakeholders.

Once it became clear that Brazil needed a bill of rights for the Internet, it also became clear that the Internet itself could and should be used as a tool for drafting the legislation. Following a series of social movements calling for such legislation, the Ministry of Justice, in partnership with academics, began an 18-month consultation process that included contributions from a variety of stakeholders. This consultation was held online and was complemented by a series of in-person events across the country. Once the bill was drafted, it was submitted to Congress, where there was an opportunity for additional online and in-person consultations. Finally, Congress approved the Marco Civil more than seven years after the first consultation.

Article 24 of the Marco Civil enforces multistakeholderism in Internet Governance, by requiring that the federal, state, and city governments must establish multi-participatory mechanisms for governance that are transparent, collaborative, and democratic, and include the government, the private sector, civil society, and the academic community. From start to finish, the approval of Marco Civil embodied this requirement, involving intense debate with several stakeholders. However, to what extent can the Marco Civil be considered a case of successful multistakeholder participation? And to what extent has it inspired advances in other multistakeholder initiatives? This articles analyses both the successes and challenges of the multistakeholder elements of the Marco Civil to address these questions. The work is based on a combination of two methodologies: a set of independent interviews conducted in 2011 with project coordinators, ${ }^{1}$ and a document and policy notes review undertaken by those running the project.

On one hand we can argue that the Marco Civil was a hybrid and transparent policymaking process that involved contributions from users, civil society organizations, telecom companies, government agencies, and universities, all side-by-side. Each contributor could see the others' contributions, and everyone placed all their cards on the table to foster open debate. On the other hand, we can argue that Marco Civil was a carefully handcrafted process where, in spite of multistakeholder forums and procedures, the success of the bill is attributable to the active role of key players that pushed the initiative forward.

One way or another the process was successful. The Marco Civil translates the principles of the Brazilian democratic Constitution to the online world. It is a victory for democracy, and for what

\footnotetext{
${ }^{1}$ Fabro Steibel and F. Beltramelli "Online Public Policy consultations" in B. Girard, Impact 2.0: New mechanisms for linking research and policy (2012, 1st Ed).
} 
it stands for. In that sense, the Brazilian Marco Civil goes in the opposite direction of other laws that have been passed recently in countries such as Turkey or Russia, which expand the powers of governments to interfere with the Internet. Brazil's law is an example of one country placing a strong emphasis on the importance of the Internet as a means of facilitating both economic development, and a rich and open public sphere.

\section{A Brief History of the Project}

The Marco Civil began in 2007 as part of a strong public reaction against a draconian cybercrime bill. The bill was nicknamed "Azeredo Law," in reference to a Senator called Eduardo Azeredo, who was the rapporteur and main supporter of the bill. If the bill had passed, it would have established penalties of up to four years in jail for anyone convicted of "jailbreaking" a mobile phone, or for anyone transferring songs from an MP3 player back onto their computer. In many ways it resembled the subsequent SOPA and PIPA legislation in the United States.

Azeredo's Law had such a broad scope that the bill would have turned millions of Internet users in Brazil into criminals. Moreover, it would have reduced opportunities for innovation by making it illegal to engage in many activities necessary for research and development. Because of this, there was a widespread negative reaction against the Azeredo Law. The first groups to raise their voice were academics, which were then followed by a strong social mobilization, including an online petition that-in a short period of time - received 150,000 signatures. Congress took notice of the reaction and, thanks to this mobilization, initiated a discussion of the topic.

There were many voices against the Azeredo Law. However, there was no clear consensus on what the alternative should be. If a criminal law was not the best way to regulate the Internet in Brazil, then what would be the best way to regulate it? In May 2007, Ronaldo Lemos wrote an article for Folha de Sao Paulo, the major newspaper in Brazil, claiming that rather than a criminal bill, Brazil should have a "civil rights framework" for the Internet: a "Marco Civil." That was the first time the term appeared in public.

The idea took off, and the Ministry of Justice picked it up. In 2008 the Ministry invited a group of professors, including Ronaldo Lemos and Carlos Affonso Souza (who were at that time leading a research institute at the Fundação Getulio Vargas), to create an open and collaborative process for drafting alternative legislation for regulating the Internet. It was clear to those involved that one could not regulate the Internet without using the Internet itself in the process. They decided to build and launch an online platform for debate and collaboration on the bill at www.culturadigital.org/marcocivil. ${ }^{3}$

The consultation was divided into two phases. The first phase involved consulting with the general public regarding a list of principles proposed for debate (i.e., freedom of expression, privacy, net neutrality, right to Internet access, intermediary liability, openness, and promotion of innovation). The second phase involved examining the proposed draft bill, article-by-article, paragraph-by-paragraph. When the consultation was over, the Government, with the support of four Ministries (Ministry of Culture, Ministry of Science and Technology, Ministry of

\footnotetext{
${ }^{2}$ Folha de São Paulo, “Internet Brasileira Precisa de Marco Regulatório Civil,” May 2007, http://tecnologia.uol.com.br/ultnot/2007/05/22/ult4213u98.jhtm.

${ }^{3}$ The archives of that dialogue are still available at this site.
} 
Communications, and Ministry of Justice) sent the bill to Congress on August 24th, 2011, and it was passed as law on April 23rd, 2014.

\section{A. The Political-Economic Settings of the Marco Civil}

In 2007, the election of President Luiz Inácio Lula da Silva was an important event for Internet issues in Brazil because he defined improving both the development of technologies and the use of the Internet as priorities for his government. Moreover, the appointment of Gilberto Gil as Ministry of Culture brought in a new perspective on Internet issues and digital culture in Brazil. For example, Gil invested in multimedia studios in the Program "Cultura Viva," developed new narratives for a Brazilian Digital Culture program inside the government, and identified public policies related to the Internet. Crucially, Gil supported the Digital Culture Forum, the platform that was used for the consultation on the draft Marco Civil legislation.

Another key reason for the passage of Marco Civil was the anger regarding U.S. surveillance activities in Brazil. When Marco Civil was ready for vote, in July 2013, Glenn Greenwald published an article in the Brazilian newspaper O Globo confirming that Brazil was a target of U.S. National Security Agency surveillance. The Brazilian government reacted quickly, and its diplomatic representatives in both the US and Brazil demanded an explanation. As a result, the issue of Internet regulation became a priority for the Brazilian government and the Marco Civil jumped to the top of the political agenda.

The government realized that the Marco Civil could be an eloquent response to the surveillance issue and position Brazil as a global leader in Internet governance and regulatory debates. On September 2013, President Dilma Rousseff made the legislation a matter of constitutional urgency, which prevented Congress from voting on any other issues until the Marco Civil vote was completed. The Marco Civil da Internet locked the agenda of the National Congress and no other vote could take place until the Chamber of Deputies (the lower house of Congress) voted on the bill. On March 25, 2014, the Chamber of Deputies approved the bill, and it was enacted in April during NETmundial.

\section{B. The Mechanics of Consultation}

Government-run consultations are not new in Brazil. In fact, law has regulated such consultations since 2000 (D4176/2000). However, apart from a few experimental initiatives, the Marco Civil was the first consultation to have such a large online focus. The Marco Civil consultation was a joint effort by the Ministry of Justice (the project's initiator) and the academic center at the Fundação Getúlio Vargas (FGV). Apart from these organizations, the project also received direct support from the Ministry of Culture, indirect assistance from other governmental bodies (i.e., the Ministry of International Relations), and ad hoc contributions from civil society organizations and Internet activists.

The general objective of the consultation was to draft an Internet law to be submitted to Congress for a vote. The bill was intended to outline a set of legal principles and rights to guide future Internet legislation, and the consultation was fully designed as a collaborative practice. The project ran from October 2009 to May 2010, and it resulted in an online forum where politicians, academics, artists, NGOs, private sector companies, individuals, and other stakeholders could post, blog, debate, and comment on the possible design of the legislation. 
The project made use of several web 2.0 tools (mainly the Wordpress platform, Twitter, RSS feeds, and blogs), and it was divided into two rounds of discussion: during the first round people were invited to comment on a white paper that offered a set of general principles that broadly oriented potential legislation. During the second round, people were invited to comment on a draft of the legislation. The first round of the debate tested a set of normative standards, predefined by those sponsoring the initiative, that were considered important to include in future legislation. In contrast, the second round focused on receiving feedback on the draft law itself.

During both periods of consultation, participants could comment on pre-defined topics, which related to one of three themes: individual and collective rights (i.e., privacy, freedom of speech, and access rights), intermediary issues (i.e., net neutrality and civil liability), and governmental directives (i.e., openness, infrastructure, and capacity building). Comments during the online consultation were not moderated, and were reviewed after posting by project leaders. Therefore, the online consultation project was a collaborative practice that invited stakeholders to bring ideas to the debate. However, the final decision-making process for incorporating those ideas rested with the initiative sponsors.

Between the first and second rounds of the consultation, project leaders met alone for two days, printed and reviewed all the contributions. These leaders then wrote the draft bill that was used for the second round of the consultation. There were some examples where stakeholder comments led to changes in the proposed text. For example, in the case of intermediary liability policies, the project leaders reversed their initial language based on online contributions. At some point during the consultation, a voting process was established, but it was quickly removed due to a perceived imbalance in the debate. In particular, older contributions tended to receive more votes than new ones, which diminished the incentive to write new comments.

\section{Successes of the Multistakeholder Process in Marco Civil}

The Marco Civil political negotiation was extremely complex, and took many years. A driving force behind the initiative was the multistakeholder process that permeated the drafting of the bill. It was clear from the start how different stakeholders positioned themselves with respect to the main policy issues. In fact, stakeholders were required to make their positions quite public. The policymakers behind the Marco Civil made efforts maximize the transparency of the stakeholders and positions. For instance, they ensured that the consultation attracted considerable media attention, they rejected contributions received by email and only acknowledged comments published online, and they required stakeholders to identify themselves and be open to public scrutiny and criticism. These efforts helped to reduce information asymmetry and facilitate negotiations. It also helped to facilitate compromises, when necessary. The Ministry of Justice, for example, had to change its position about log records; ISPs and telecommunications companies had to agree to disagree on net neutrality; and civil society was indirectly forced to review their position with regards to the removal of revenge porn.

To illustrate the point regarding transparency, Table 1 lists the main controversies of the Marco Civil debate and identifies the positions for each of the main stakeholders ${ }^{4}$. Telecommunications

\footnotetext{
${ }^{4}$ Data portrayed in Tables 1, 2 and 3 are based on previous analysis of the article authors during the years of drafting and approval of Marco Civil.
} 
companies, for example, were against or neutral during the whole process around the key issues of the Marco Civil, while civil society was in favor of four of the six analyzed issues, and neutral about the other two. The data comparing stakeholders' positions suggests, when compared to the text of the final bill, that transparency with respect to stakeholder positions forced trade-offs and brought transparency to negotiations.

\begin{tabular}{|c|c|c|c|c|c|c|c|}
\hline Issues/Actors & $\begin{array}{c}\text { Net } \\
\text { Neutrality }\end{array}$ & $\begin{array}{c}\text { Highly } \\
\text { Enhanced } \\
\text { Privacy }\end{array}$ & $\begin{array}{c}\text { Safe } \\
\text { Harbor } \\
\text { for Speech }\end{array}$ & $\begin{array}{c}\text { Data } \\
\text { Retention }\end{array}$ & $\begin{array}{l}\text { Forced data } \\
\text { Localization }\end{array}$ & $\begin{array}{c}\text { Safe } \\
\text { Harbor } \\
\text { for } \\
\text { Copyright }\end{array}$ & $\begin{array}{c}\text { Express } \\
\text { Removal } \\
\text { for } \\
\text { Revenge } \\
\text { Porn }\end{array}$ \\
\hline Telcos & Against & Against & Neutral & Neutral & Neutral & Neutral & Neutral \\
\hline Civil Society & For & For & For & Against & Against & For & Against \\
\hline $\begin{array}{l}\text { Global Internet } \\
\text { Companies }\end{array}$ & Neutral & Against & For & Neutral & Against & For & Neutral \\
\hline $\begin{array}{l}\text { Brazilian Internet } \\
\text { Companies }\end{array}$ & For & Against & For & Against & Against & Against & Neutral \\
\hline Broadcast sector & For & For & For & Neutral & Neutral & Against & Neutral \\
\hline $\begin{array}{c}\text { Government } \\
\text { (Executive branch) }\end{array}$ & For & Neutral & Neutral & For & For & Neutral & For \\
\hline $\begin{array}{c}\text { Law enforcement } \\
\text { lawyers/ Federal } \\
\text { Police }\end{array}$ & Neutral & Against & Against & For & For & Against & For \\
\hline Passed legislation & Passed & Passed & Passed & Passed & Rejected & Rejected & Passed \\
\hline
\end{tabular}

\section{Table 1. Summary of stakeholder actors' positions vs. Marco Civil key issues}

Tables 2 and 3 suggest that the multistakeholder cannot by itself explain the results of the policy process. What we mean by that is that no stakeholder achieved their desired result in every issue, and there was no policy issue where there was a clear alignment among all stakeholders. Using the data from Table 1 regarding the position of each actor for each issue, we can give a value of +1 for in favor, 0 for neutral, and -1 for against. The results of this appear in Table 2. From Table 2 we notice that out of the seven issues identified, the telcos and Brazilian Internet companies were the stakeholders who most opposed the Marco Civil. The telcos were mostly neutral and opposed the net neutrality and privacy provision. And the Brazilian Internet companies both supported and opposed two provisions of the bill. As expected, the government was the most supportive of the bill $(+4)$, never positioning itself against the main issues, but it lost in regards of the issue of Forced data localization. 


\begin{tabular}{lcccc}
\hline \multicolumn{1}{c}{ Issues/Actors } & For (+1) & Neutral (0) & Against (-1) & Actor Sum \\
\hline Telcos & 0 & 5 & 2 & $\mathbf{- 2}$ \\
Civil Society & 4 & 0 & 3 & $\mathbf{1}$ \\
Global Internet Companies & 2 & 3 & 2 & $\mathbf{0}$ \\
Brazilian Internet Companies & 2 & 1 & 1 & $\mathbf{- 2}$ \\
Broadcast sector & 3 & 3 & 0 & $\mathbf{2}$ \\
Government & 4 & 3 & 3 & $\mathbf{4}$ \\
Law enforcement lawyers/ Federal Police & 3 & 1 & $\mathbf{1 5}$ & $\mathbf{0}$ \\
\hline Total & $\mathbf{1 8}$ & $\mathbf{1 6}$ & & \\
\hline
\end{tabular}

Table 2. Sum of positions for, neutral or against vs. actors

Table 3 illustrates that the stakeholders strongly disagreed about the general issues. This means that no single issue was a major point of consensus amongst all stakeholders, with a distribution of opinions between all possible positions emerging as the most common pattern. Reading the three tables together, we can see, for example, the issue that faced the most opposition (highly enhanced privacy) and the three that found most support (net neutrality, safe harbor for speech and express removal of revenge porn) were all included in the approved bill.

\begin{tabular}{|c|c|c|c|c|c|c|c|}
\hline Issues/Actors & $\begin{array}{c}\text { Net } \\
\text { Neutrality }\end{array}$ & $\begin{array}{l}\text { Highly } \\
\text { Enhanced } \\
\text { Privacy }\end{array}$ & $\begin{array}{c}\text { Safe } \\
\text { Harbor for } \\
\text { Speech }\end{array}$ & $\begin{array}{c}\text { Data } \\
\text { Retention }\end{array}$ & $\begin{array}{l}\text { Forced data } \\
\text { Localization }\end{array}$ & $\begin{array}{c}\text { Safe } \\
\text { Harbor } \\
\text { for } \\
\text { Copyright }\end{array}$ & $\begin{array}{c}\text { Express } \\
\text { Removal } \\
\text { for } \\
\text { Revenge } \\
\text { Porn }\end{array}$ \\
\hline For $(+1)$ & 4 & 2 & 4 & 2 & 2 & 2 & 4 \\
\hline Neutral (0) & 2 & 1 & 2 & 3 & 2 & 2 & 2 \\
\hline Against (-1) & 1 & 4 & 1 & 2 & 3 & 3 & 1 \\
\hline Issue sum & 3 & -2 & 3 & 0 & -1 & -1 & 3 \\
\hline Passed legislation & Passed & Passed & Passed & Passed & Rejected & Rejected & Passed \\
\hline
\end{tabular}

Table 3. Sum of positions for, neutral or against vs. issues

The tables together illustrate the complexity of the Marco Civil negotiation, both in terms of the number of parties involved and in terms of the variety of issues discussed. That said, we argue that although a multistakeholder approach may offer transparency to the policymaking process, it does not necessarily help one predict the outcome of the process. The Marco Civil was an important achievement not only for Brazil but also globally. It is important because of its substance and also its process, representing the intrinsic connection between the open and collaborative process it used, and the substantive results it achieved. 


\section{Challenges of Multistakeholderism in Marco Civil}

Often, "multistakeholderism," is a commonplace mantra, but multistakeholder processes alone were not sufficient in the case of the Marco Civil. In order to solve the contradictions and disputes involved in the Marco Civil it took an intensive negotiation effort. Multistakeholder processes throughout the Marco Civil drafting process were helpful (and important) starting places. However, in order to achieve effective results, it was necessary to engage in debates and negotiations that required actors to put aside radicalism and polarization, and to be ready to compromise during the process. This is one of the main lessons of the Marco Civil ${ }^{5}$ process.

The first challenge of the Marco Civil was attracting a sufficient number of contributors, and prompting them to engage in positive discussion. Based on an extensive analysis of 15 years of online consultations, we can report that the overall volume of participation in the Marco Civil consultations, as measured by number of authors and comments, is significant ${ }^{6}$. More than two thousand comments were posted during the consultations. Excluding the ones that offered little content (e.g., yes/no simple statements, Twitter links, or external references without comments), the online consultations received 1,507 comments and attracted 287 participants (only 22 of them engaging in both phases of consultation).

\begin{tabular}{lccc} 
& Phase 1 & Phase 2 & Total \\
\hline & & $\#$ & $\#$ \\
\hline Number of contributions & & & \\
\hline Number of comments & 623 & 884 & 1,507 \\
\hline Duration of consultation & 99 days & 52 days & 151 days \\
\hline Average of comments per day & 6.3 & 17.0 & 10.0 \\
\hline
\end{tabular}

\begin{tabular}{lccc}
\hline Number of contributors & & & \\
\hline Round $1+2$ authors (including returning commenters) & 127 & 160 & 287 \\
\hline All vs. Returning authors & $127(0)$ & $160(22)$ & $287(22)$ \\
\hline
\end{tabular}

\section{Table 4. Marco Civil public consultation numbers}

A second challenge of the Marco Civil process was ensuring that all of the topics open for debate received comments and feedback. When we look at the individual forums we find that not all sections of the Marco Civil were discussed in significant detail (even if they were open for

\footnotetext{
${ }^{5}$ Data portrayed in Tables 4 and 5 are based on previous analysis of the authors, in particular see Fabro Steibel and F. Beltramelli “Online Public Policy consultations" in B. Girard, et al., eds., Impact 2.0: New Mechanisms for Linking Research and Policy (2012, 1st Ed); Fundación Comuca, F. Steibel, and E. Estevez, Designing Web 2.0 Tools for Online Public Consultation (forthcoming 2014).

${ }^{6}$ Stephen Coleman and Peter Shane, Connecting Democracy: Online Consultation and the Flow of Political Communication (Cambridge: MIT Press, 2012).
} 
debate). A policy forum is defined as an independent comment section within the consultation, so that those who participate in that forum see the other contributions left in the same forum. As Table 5 suggests, out of the 127 policy forums open for discussion during the Marco Civil (rounds 1 and 2 combined), 13\% of those forums received no comments, $4 \%$ of the forums received more than 25 comments, and in $25 \%$ of forums more than 25 contributors participated. However, the overall picture suggests that the Marco Civil process mostly involved forums with around 10 to 25 comments or which involved no more than five authors.

\begin{tabular}{|c|c|c|}
\hline & \multicolumn{2}{|c|}{ Total } \\
\hline & \# & $\%$ \\
\hline \multicolumn{3}{|l|}{ Number of comments per forum } \\
\hline No comments received & 17 & $13 \%$ \\
\hline From 1 to 9 comments & 54 & $43 \%$ \\
\hline From 10 to 25 comments & 62 & $49 \%$ \\
\hline More than 25 comments & 5 & $4 \%$ \\
\hline \multicolumn{3}{|l|}{ Number of authors per forum } \\
\hline No authors & 17 & $13 \%$ \\
\hline No authors or up to 5 authors & 62 & $49 \%$ \\
\hline From 6 to 25 authors & 33 & $26 \%$ \\
\hline More than 25 authors & 32 & $25 \%$ \\
\hline Total number of forums in Phase 1 and 2 & 127 & $100 \%$ \\
\hline
\end{tabular}

Table 5. Marco Civil policy forums' profile

A third challenge faced by the Marco Civil was tracking support and opposition to various issues. We analyze the variation in the number of comments that presented support, or opposition to the specific issue under consideration or diverged from the proposed issue. In our analysis, we find that most comments diverged from the proposed language. Divergence happened primarily in two main ways: (1) the author ignored the suggested policy and suggested an alternative to it; or (2) the author ignores the suggested policy instead supports, opposes, or diverges from another author's contribution. Taking into consideration that three out of four comments fall into this category (75\%), we can argue that the Marco Civil prompted a vigorous debate where the original policy proposal was briefly reviewed and then stakeholders offered a vast range of alternative positions. 


\begin{tabular}{lll}
\hline Comment's position towards suggested consultation & MC & Percent \\
\hline Support & 168 & $11 \%$ \\
Opposition & 201 & $14 \%$ \\
Divergence & 1,138 & $75 \%$ \\
\hline Total of comments received & 1,507 & $100 \%$ \\
\hline
\end{tabular}

Table 6. Contributors' support for Marco Civil policies

\section{Conclusion}

Taken together, the three challenges (a significant but still limited number of contributions; policy forums with a limited number of comments and contributors; and the divergence of contributions) it might seem that the Marco Civil was a unique experience. The Marco Civil process was certainly experimental ${ }^{7}$. However, many elements of the process were less unique: (1) the online consultation was conducted in parallel with in-person consultations across the country; (2) policy debates occurred in traditional media; (3) many parliamentary discussions and online consultations were run by the Congress; and (4) there were several parallel debates in civil society, the business sector, the technical community, and academia.

The challenges faced by the Marco Civil illustrate the importance of a multistakeholder process. A multistakeholder process does not necessarily provide better policies outcomes than a nonmultistakeholder process, but in the case of Marco Civil, it enabled a more accessible, participatory, and publically accountable policymaking process. As we describe above, the multistakeholder process in the Marco Civil cannot explain the final results of the bill passed in Congress. Nonetheless, the process gave transparency to the positions of the main stakeholders on the key policies, encouraging stakeholders to negotiate and collaborate.

The online consultations of the Marco Civil process ultimately required a combination of six factors: (a) a government-based institution with a real interest in direct public participation; (b) an active online community with a strong interest in the topic under discussion; (c) an active think tank institution willing to bring its own expertise and influence to the project; (d) a web 2.0 interface capable of engaging policymakers and citizens in a coherent narrative structure for deliberation; (e) policy makers able to identify the key issues for deliberation, determine how technology would be used to mediate deliberation, and to translate contributions into a properly formatted legal policy document; and (f) sufficient resources, backed by in this case by the Ministry of Justice itself, to pay for the project and promote it online and offline.

That being said, the process of the Marco Civil consultations involved a complex culture of perpetual experimentation. To some extent, the online consultations resembled a completely new environment for policy making. For example: policy makers were required to understand the essence of tools such as Twitter and email, and decide if they were suitable for use in the consultation. Based on policy makers' previous experiences with offline policy forums, they

\footnotetext{
${ }^{7}$ T. O’Reilly, “Government as a Platform” in D. Lathrop and L. Ruma, eds., Open Government: Collaboration, Transparency, and Participation in Practice (O’Reilly Media: Sebastapol, 2010).
} 
were willing to avoid the one-to-few communication flow in favor of a many-to-many flow online. For example, they decided (after a trial-and-error period) to use Twitter (but not email) to interact with the general public.

Ultimately the consultation process supported the eventual enactment of the law. Although it was not possible for participants in the consultations to control the ultimate outcome of the Marco Civil, the fact that the drafted bill reflected the public contributions of several stakeholders made it less likely that the text would be significantly revised after the consultations. Additionally, the community from the consultations contributed to a robust and lasting debate around the bill, which extended until Congress approved the bill and even after passage. 


\section{Appendix J: \\ The Global Multistakeholder Meeting on the Future of Internet Governance (NETmundial)}




\title{
NoC Internet Governance Case Studies Series: The Global Multistakeholder Meeting on the Future of Internet Governance (NETmundial)
}

\author{
Marilia Maciel, ${ }^{1}$ Nicolo Zingales, and Daniel Fink ${ }^{2}$ \\ Center for Technology and Society of the Getulio Vargas Foundation
}

Editorial Note: Context, Character, and Purpose of the Case Study

This case study is part of a globally coordinated, independent academic research pilot project by the Global Network of Interdisciplinary Internet \& Society Research Centers (NoC). Facilitated by the Berkman Center for Internet \& Society at Harvard University, this study examines existing multistakeholder governance groups with the goal of informing the future evolution of the Internet governance ecosystem. Building upon the NETmundial Principles and Roadmap, it contributes to current policy debates at the international level, including the Internet Governance Forum, the NETmundial Initiative, and other organizations and efforts.

Internet governance is an increasingly complex concept that operates at multiple levels and in different dimensions, making it necessary to have a better understanding of both how multistakeholder governance groups operate and how they best achieve their goals. With this need in mind, at a point where the future of Internet governance is being re-envisioned, colleagues from several $\mathrm{NoC}$ institutions around the world have written twelve case studies examining a geographically and topically diverse set of local, national, and international governance models, components, and mechanisms from within and outside of the sphere of Internet governance. Key findings from these cases are summarized in a synthesis paper, which aims to deepen our understanding of the formation, operation, and critical success factors of governance groups and even challenge conventional thinking.

The research, based on twelve case studies, suggests that there is no single best-fit model for multistakeholder governance groups that can be applied in all situations. Rather, it reveals a range of approaches, mechanisms, and tools available for both the formation and operation of such groups. The analysis demonstrates that the success of governance groups depends to a large degree on the careful selection, deployment, and management of suitable instruments from this "toolbox." As governance groups pass through different phases of operation, conveners and facilitators must remain alert to changes in circumstances that necessitate adjustments to approaches, mechanisms, and tools that they deploy in order to address evolving challenges from inside and outside. This case study provides insights into how those instruments can be deployed and adjusted over time within such groups, and highlights how their interactions with important contextual factors may be successfully managed within given resource restraints.

The research effort is grounded in a diversity of global perspectives and collaborative research techniques. Adhering to objective and independent academic standards, it aspires to be useful, actionable, and timely for policymakers and stakeholders. More broadly, the Network of Centers seeks to contribute to a more generalized vision and longer-term strategy for academia regarding its roles in research, facilitation and convening, and education in and communication about the Internet age.

For additional information on the initiative, please contact Urs Gasser, Berkman Center for Internet \& Society, at ugasser@cyber.law.harvard.edu.

\footnotetext{
${ }^{1}$ Marília was a member of the Executive Multistakeholder Committee, which played a central role in the organization of NETmundial.

${ }^{2}$ Daniel was the Executive Director of the NETmundial Secretariat.
} 


\begin{abstract}
This case study examines NETmundial, the Global Multistakeholder Meeting on the Future of Internet Governance, which was held in Sao Paulo, Brazil on April 23 and 24 of 2014. The meeting was convened by 1net, a coalition of stakeholder groups involved in Internet governance discussions, in partnership with the Brazilian Internet Steering Committee (CGI.br), in response to revelations of mass surveillance of communications by the United States. It sought to develop a set of universally acceptable Internet governance principles as well as a way forward for the evolution of the Internet governance system, which together could serve as a framework for the governance and use of the Internet. It convened 930 participants from 110 different countries, representing civil society, the private sector, academia, the technical community, governments and intergovernmental organizations, as well as over 1000 remote participants from 23 countries around the globe. It also employed a content contribution platform that sought to crowd source inputs from stakeholders for the production of the outcome document. The meeting served as a demonstration of the multistakeholder process in action: in the production of the outcome document, stakeholders with a diverse range of backgrounds and interests collectively negotiated the inclusion or exclusion of highly sensitive and complex issues. While the process of achieving rough consensus involved sometimes messy debates and there were procedural imperfections, the case is informative for its structured production of bottom-up multistakeholder outcomes.
\end{abstract}




\section{Table of Contents}

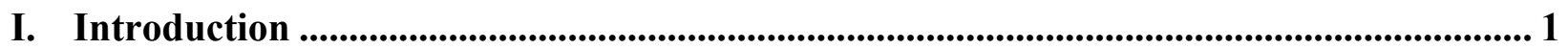

II. Values and Function .................................................................................................. 3

III. Organizational Model and Structure......................................................................................... 5

IV. Participation and Decision-Making Structures................................................................ 10

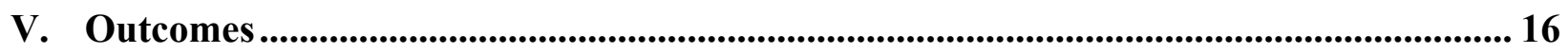

A. Political Outcomes of the Meeting ......................................................................................................... 16

B. Outcome Document.............................................................................................................................. 17

VI. Best Practices and Templates.............................................................................................. 19 


\section{Introduction}

The Global Multistakeholder Meeting on the Future of Internet Governance (NETmundial) was held on April 23rd and 24th, 2014 in São Paulo, Brazil. The event was organized by means of a partnership between the Brazilian Internet Steering Committee (CGI.br) ${ }^{3}$ and 1net, ${ }^{4}$ a coalition that gathers actors from the various stakeholder groups involved in Internet governance discussions.

Revelations of mass surveillance of digital communications were a catalyst for the decision to convene NETmundial, although the issue of surveillance was not initially included in the agenda of the meeting. In September 2013, Brazilian President Dilma Rousseff gave a speech at the opening of the 68th Session of the United Nations General Assembly, in which she criticized policies of mass surveillance. ${ }^{5}$ President Rousseff also mentioned the need to develop a framework for the governance and use of the Internet, and to create mechanisms to ensure basic principles are guaranteed, such as privacy, freedom of speech, and net neutrality. The following month she received a visit from Fadi Chehadé, Chief Executive Officer of the Internet Corporation for Assigned Names and Numbers (ICANN). After this conversation, the global meeting was jointly announced. ${ }^{6}$ This partnership helped both actors advance some of their objectives.

Brazil is one of the most politically active developing countries in Internet governance discussions. It has consistently stressed the need for a "multilateral, transparent and democratic governance of the Internet", 7 advocating for changes, especially with regards to Internet

\footnotetext{
${ }^{3}$ Brazil has extensive experience in multistakeholder discussions of Internet-related issues. The primary example is the Brazilian Internet Steering Committee (CGI.br), which currently encompasses 21 members. Ten are governmental representatives and 11 are non-governmental actors. Among other activities, CGI.br is responsible for coordinating joint actions for the proposal of policies and procedures related to the regulation of Internet activities, deliberating on any matters referred to it that relate to Internet services in Brazil, and adopting the necessary administrative and operational procedures for the management of the Internet in Brazil in accordance with accepted international standards. CGI.br. "About CGI.br.” Accessed January 6, 2015. http://cgi.br/about/.

${ }^{4} 1$ net was created at the end of 2013 with the purpose of providing an inclusive and open platform for discussion of Internet governance matters among interested actors. The platform was launched quickly so it could facilitate the involvement of stakeholders in the preparatory process of NETmundial. NETmundial was the first and most important topic in the agenda of 1 net so far and participation in the mailing list has declined following the event. 1net. "1net Homepage." Accessed January 6, 2015. http://1net.org/.

${ }^{5}$ Dilma Rousseff, "Statement By H. E. Dilma Rousseff, President Of The Federative Republic Of Brazil, at The Opening Of The General Debate Of The 68th Session Of The United Nations General Assembly, 2013," September 24, 2013, http://gadebate.un.org/sites/default/files/gastatements/68/BR_en.pdf.

6 "Brasil vai sediar encontro mundial sobre governança da internet em 2014," Blog do Planalto, October 9, 2013, http://blog.planalto.gov.br/brasil-vai-sediar-encontro-mundial-sobre-governanca-da-internet-em-2014/.

${ }^{7}$ This expression emerged for the first time during the Regional Ministerial Preparatory Conference of Latin America and the Caribbean for the World Summit on the Information Society (WSIS), as part of the Bávaro Declaration. The Tunis Agenda, one of the documents approved by the end of WSIS, mentions that Internet governance should be "multilateral, transparent and democratic, with the full involvement of governments, the private sector, civil society and international organizations." In the $68^{\text {th }}$ UNGA president Dilma Rousseff called for "Open, multilateral and democratic governance, carried out with transparency by stimulating collective creativity and the participation of society, Governments and the private sector."
} 
governance institutions and the United States' perceived unilateral oversight thereof. ${ }^{8}$ In some interviews with the press, the chair of the NETmundial meeting, Virgilio Almeida, explicitly mentioned the goal of strengthening the multistakeholder model ${ }^{9}$ and introducing changes to the contractual relationship between ICANN and the United States Department of Commerce. ${ }^{10}$ Both topics - the need for democratic and multistakeholder governance and the transition of the stewardship of the IANA functions - were intensively discussed at NETmundial.

For ICANN and the I* organizations (read "I star"), ${ }^{11}$ the meeting was viewed as an opportunity to counterbalance government-centric multilateral proposals for improving the Internet governance ecosystem. Those proposals emerged in the World Conference on International Telecommunications (WCIT), held by the International Telecommunications Union (ITU) in 2012, and in the ongoing World Summit on the Information Society (WSIS) +10 process. $^{12}$ During WCIT, some participants attempted to include the Internet within the ITU's scope over telecommunications and introduced a non-binding resolution that blurred the lines between telecommunications and the Internet. There has been a similar attempt to strengthen governmentcentric multilateral governance over the Internet. For example, in July 2014, a UN resolution about the goals and modalities for participation at WSIS +10 was approved, ${ }^{13}$ calling for the establishment of an intergovernmental negotiation process that should include informal consultation with other relevant stakeholders.

A lack of alternative proposals raised concerns that the pressure to move towards more traditional, top-down inter-governmental arrangements would increase. ${ }^{14}$ NETmundial was seen as an opportunity to start the discussion about other options for the evolution of the Internet

\footnotetext{
${ }^{8}$ Everton Lucero, Governança da Internet: Aspectos da formação de um Regime Global e Oportunidades para a Ação Diplomática, (Barsília: Fundação Alexandre de Gusmão, 2011), accessed January 6, 2015, http://www.funag.gov.br/biblioteca/dmdocuments/0514.pdf.

9 “Conferência Multissetorial sobre Internet no Brasil deve ter 150 países participantes," Teletime, January 28, 2014, http://www.teletime.com.br/28/01/2014/conferencia-multissetorial-no-brasil-deve-ter-150-paisesparticipantes/tt/366869/news.aspx.

${ }^{10}$ Taíssa Stivanin, "Brasil sedia conferência mundial sobre governança na Internet," RFI, April 23, 2014, http://www.portugues.rfi.fr/geral/20140423-brasil-sedia-conferencia-mundial-sobre-governanca-na-internet.

${ }^{11}$ The I* is a group of organizations responsible for coordination of the Internet's technical infrastructure. The organizations include: the Internet Corporation for Assigned Names and Numbers, the Internet Engineering Task Force, the Internet Architecture Board, the World Wide Web Consortium, the Internet Society, and the five regional Internet address registries. These registries are: African Network Information Center (AFRINIC), American Registry for Internet Numbers (ARIN), Asia-Pacific Network Information Centre (APNIC), Latin America and Caribbean Internet Addresses Registry (LACNIC), and Réseaux IP Européens Network Coordination Centre (RIPENCC).

${ }^{12}$ The WSIS was convened in two phases. The first meeting took place in Geneva in 2003, with the aim to foster the political will to advance an Information Society for all. The main documents approved were the Geneva Declaration of Principles and the Geneva Plan of Action. The second phase took place in Tunis in 2005, with the aim to put Geneva's Plan of Action into motion as well as to find solutions and reach agreements in the fields of Internet governance, financing mechanisms, and follow-up on the implementation of the Geneva documents. The main documents approved on that occasion were the Tunis Commitment and the Tunis Agenda for the Information Society.

${ }^{13}$ Un General Assembly, Resolution A/Res/68/302, "Modalities for the overall review by the General Assembly of the implementation of the outcomes of the World Summit on the Information Society," August 13, 2014, http://www.un.org/en/ga/search/view_doc.asp?symbol=A/RES/68/302.

${ }^{14}$ Stéphane Van Gelder, "ICANN Explains Brazil Meeting Initiative," CircleID, October, 23, 2013, http://www.circleid.com/posts/20131023_icann_explains_brazil_meeting_initiative/.
} 
governance ecosystem and to gain a better understanding of what a new international consensus might look like. ${ }^{15}$

This case study will examine how NETmundial was organized and operated, with a focus on the process of drafting the outcome document, named the NETmundial Multistakeholder Statement. The process of drafting the document in a bottom-up and multistakeholder way is a key legacy of the event. It is important, therefore, to document and analyze the methodology of NETmundial, not only to capture what worked well, but also to identify aspects for improvement of this unique experiment.

\section{Values and Function}

It is impossible to extricate NETmundial from the political context in which it was convened, marked by revelations of mass surveillance. During the announcement of NETmundial, Chehadé affirmed that "the trust in the global Internet has been punctured and now it's time to restore this trust through leadership and institutions that can make that happen."16 The perception that the chain of trust had been broken "created a sense of urgency to review current Internet governance arrangements." ${ }^{17}$ It is possible to affirm, therefore, that one of the goals of NETmundial was to restore a positive governance environment that would foster trust, cooperation and dialogue about common challenges.

NETmundial was explicitly aimed at addressing two problems that directly inspired its agenda items: the need to identify a set of universally acceptable Internet governance principles and the need to propose a way forward for the evolution of the Internet governance ecosystem. These concrete goals were defined in a meeting between the Brazilian government and representatives from the I* organizations. ${ }^{18}$ They were further detailed by the members of the Executive Multistakeholder Committee of NETmundial (EMC) in two short paragraphs published on the NETmundial website.

Principles have been historically relevant for the development of Internet governance. The WSIS Declaration of Principles, for example, has been called a constitution for the information age. ${ }^{19}$ In recent years, sets of principles aiming to guide Internet governance and policy development have been put forward by several organizations, leading to some to call this a "constitutional moment" for global Internet governance. ${ }^{20}$ This bevy of principles includes those developed by the Council of Europe, OECD, European Union, the G-8, by the government of the United States, and by CGI.br. Efforts to map, compare, and identify commonalities among sets of

\footnotetext{
${ }^{15}$ Markus Kummer, "The Ever Evolving Landscape of Internet Governance," in Stakes are High: Essays on Brazil and the Future of the Global Internet (Internet Policy Observatory, April 2014) http://globalnetpolicy.org/wpcontent/uploads/2014/04/StakesAreHigh_BrazilNETmundial_final.pdf\#markuskummer.

${ }^{16}$ Van Gelder, "ICANN Explains."

${ }^{17}$ Kummer, "Ever Evolving Landscape."

${ }^{18}$ Adiel Akplogan, e-mail to I-coordination list, November, 18, 2014, https://www.nro.net/pipermail/icoordination/2013-November/000077.html.

${ }^{19}$ Daniel Stauffacher and Wolfgang Kleinwächter, eds., The World Summit On The Information Society: Moving From The Past Into The Future (New York: The United Nations ICT Task Force, 2005), http://www.icvolunteers.org/files/wsis_past2future_ebook1.pdf.

${ }^{20}$ Wolfgang Kleinwächter, "Internet principle hype: how soft law is used to regulate the Internet," .NXT: Internet Policy and Governance Dissected, July 25, 2011, http://news.dot-nxt.com/2011/07/27/internet-principle-hype-anon.
} 
principles have recently been undertaken, showing that there is considerable degree of convergence among them. ${ }^{21}$

Despite the similarity between documents, these sets of principles have yet to combine both global scope and multistakeholder support: most of them have been recognized only by a particular country or region and have not been developed with the involvement of all stakeholders. One of the aims of NETmundial was to "identify a set of universal Internet governance principles to be promoted as a global inspiration for the evolution of the Internet worldwide. Those principles should support the perspective of the Internet as a global platform for social, economic and human development and a catalyst to exercise human rights. ${ }^{, 22}$ In order to accomplish that, NETmundial participants were encouraged to build upon existing sets of principles, which were listed and made available on the website. ${ }^{23}$

The second agenda item of NETmundial, setting out a roadmap for the future of Internet governance, was less neatly defined, probably reflecting the diversity of topics it encompassed. The improvement of the ecosystem has been debated in several UN forums, such as the Internet Governance Forum (IGF), ${ }^{24}$ in the International Telecommunications Union (ITU), in the WSIS process, and in the UN General Assembly. Discussions have also taken place in ad-hoc groups, such as the Panel on Global Internet Cooperation and Governance Mechanisms and in the Global Commission on Internet Governance, created by CIGI and Chatham House.

Given the multitude of parallel forums and the relative paralysis on the issue in the UN, a goal of NETmundial was "to energize discussion and pursue agreements for the way forward (...) to evolve and extend Internet governance institutions and networks to address current and emerging issues." ${ }^{25}$ Given the broad scope of topics, the EMC suggested a set of questions to facilitate contributions from participants. ${ }^{26}$

In addition to these explicit goals, the convening of NETmundial - a new event in an already crowded agenda of Internet governance meetings and forums - revealed the existence of a gap. There was no global multistakeholder space for actually drafting outcome documents. NETmundial was an alternative way to address this problem. The meeting was convened under the expectation that it should present conclusions and a concrete outcome. According to a report presented by the chair of 1 net, a critical element for the success of the meeting would be "a final

\footnotetext{
${ }^{21}$ See Jeonghyun Baak and Carolina Rossini, "Issue Comparison of Major Declarations on Internet Freedom," Summer 2013, http://bestbits.net/issue-comparison-of-major-declarations-on-internet-freedom/.

${ }_{22}$ Executive Multistakeholder Committee (EMC), NETmundial, "Executive report Executive Multistakeholder Committee (EMC), 1st meeting," January, 27, 2014, http://netmundial.br/wp-content/uploads/2014/01/EMC-Jan-

27_Executive-report.pdf.

23 "Links to Internet Governance Principles," NETmundial, http://content.netmundial.br/internet-governanceprinciples/.

${ }^{24}$ The IGF work took place through working groups created under the auspices of the UN Commission on Science and Technology for Development, such as the Working Group on IGF improvements and the Working Group on Enhanced Cooperation.

${ }^{25}$ EMC, "1st meeting."

${ }^{26}$ The guiding questions were: a) Is there a forum or Internet governance body that develops policy or technical outcomes involved in these issues?; b) If there is, how and why are these issues not being adequately dealt with by that forum or organization?; c) What are the possible responses to the challenges posed by these issues?; d) How will the possible responses proposed ensure the stability, resilience and efficiency and also comply with principles of equitable multistakeholder participation, accountability, transparency and predictability?
} 
joint declaration of internet principles and an institutional framework for multistakeholder Internet governance. The declaration should aim to be concrete/practical, linked to prior/current Internet governance initiatives, and hopefully include some next steps."${ }^{, 27}$

It was a key objective of NETmundial that this outcome document would be developed through a bottom-up multistakeholder process. However, the methodology for drafting the document was not clear from the outset, with the exception of the fact that inputs should be channeled through online consultation in addition to face-to-face discussions during the two days of NETmundial.

\section{Organizational Model and Structure}

The organization of NETmundial was carried out by a multistakeholder board of chairs, appointed by the Chairman of the meeting to represent the four key communities (civil society, the technical community, academia, and the private sector), ${ }^{28}$ and four organizational committees. The structure and the methodology for the composition of the committees was jointly determined by Brazilian government representatives and the $\mathrm{I}^{*}$ organizations. ${ }^{29}$ It is worth noting the prominent role that the I* organizations had on the shape of the meeting. Inet coorganized the meeting and its chair, Adiel Akplogan, represents AFRINIC among the I* organizations. ICANN, which is also part of the I* organizations, had a prominent role from the outset, financing part of the logistical expenses and working in close connection with the Brazilian government and CGI.br on the organization of the meeting. It is possible to say, therefore, that this subset of the technical community was the most influential among nongovernmental stakeholders when it came to the initial formation of NETmundial.

The mandates of each committee were the following: ${ }^{30}$

1. High-Level Multistakeholder Committee (HLMC): Responsible for conducting the political articulation and fostering the involvement of the international community.

2. Executive Multistakeholder Committee (EMC): Responsible for organizing the event, including the agenda and execution, and for the review of the proposals from participants;

3. Logistics and Organizational Committee (LOC): Responsible for overseeing every logistical aspect of the meeting;

4. Council of Governmental Advisors (CGA): Open to all governments willing to contribute to the meeting.

\footnotetext{
${ }^{27}$ Akplogan, e-mail.

${ }^{28}$ The chair, Virgílio Almeida, was supported by Jeanette Hofmann (academic community), Subi Chaturvedi (civil society), Fadi Chehadé (technical community), and Andile Ngcaba (private sector).

${ }^{29}$ Akplogan, e-mail.

30 “Committees," NETmundial, http://netmundial.br/\#committees.
} 


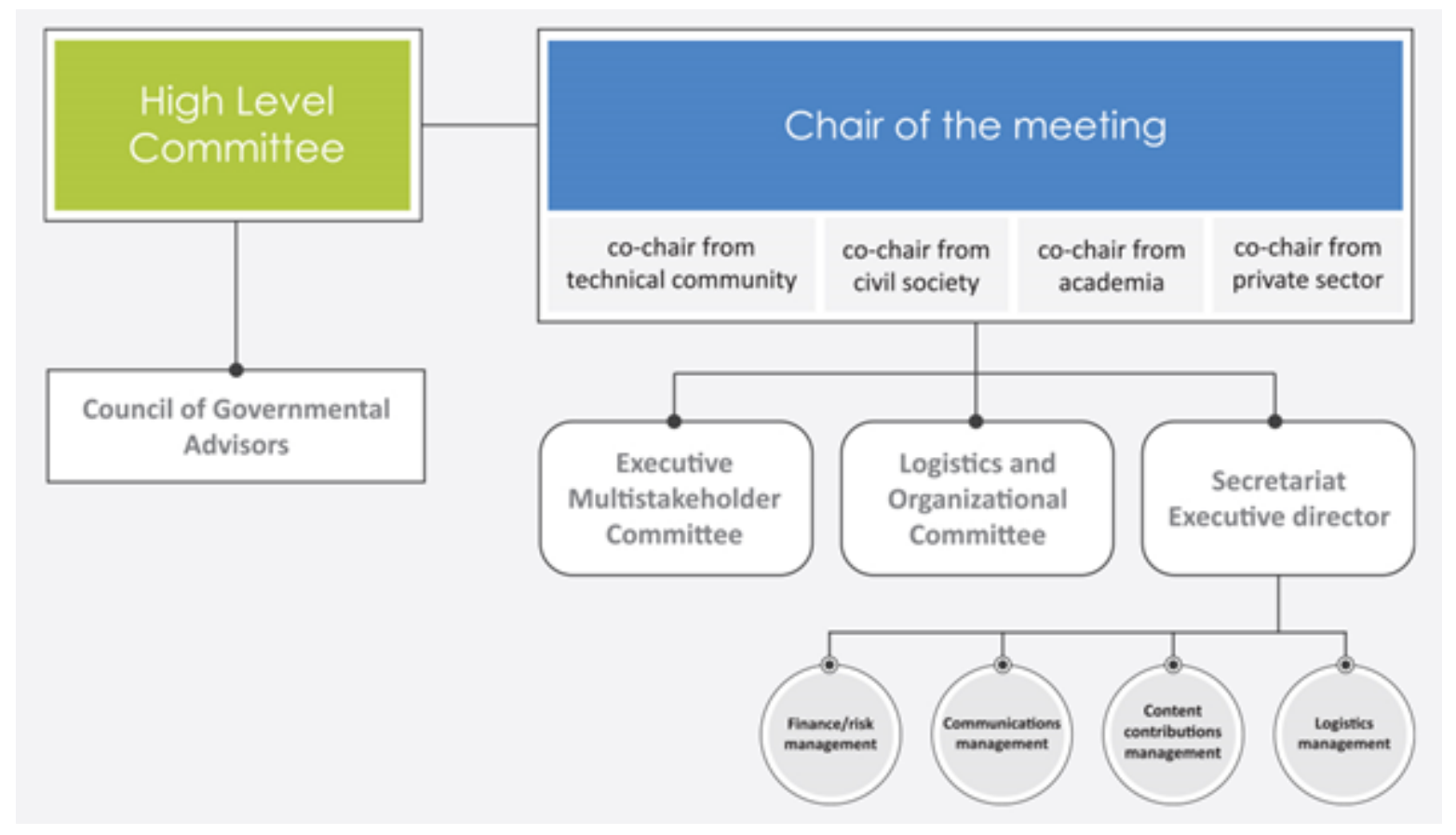

Figure 1. The organization of NETmundial.

The HLMC and the EMC were multistakeholder bodies. By contrast, the CGA was composed only of governments, and the LOC had representatives from the Brazilian government, CGI.br, ICANN, and 1net. In addition, an Executive Secretariat was established to provide support to all committee activities. It was composed of a team of five people, who were the only personnel with full-time dedication to the event and who were financially supported by the event. This group worked on site at the Brazilian Internet Steering Committee (CGI.br) in São Paulo.

The HLMC included 27 members: one chair (the Brazilian Minister for Communications), 12 representatives of States as co-hosts (invited by the Brazilian Ministry of Communications), ${ }^{31}$ two from international organizations (appointed by the UN Secretary General), and 12 from the multistakeholder community (three each from civil society, the private sector, academia, and the technical community) nominated through 1 net.

\footnotetext{
${ }^{31}$ The twelve countries were: Argentina, Brazil, France, Ghana, Germany, India, Indonesia, South Africa, South Korea, Tunisia, Turkey and the United States of America. Each continent was granted three slots with the recommendation of involving other governments from their region.
} 


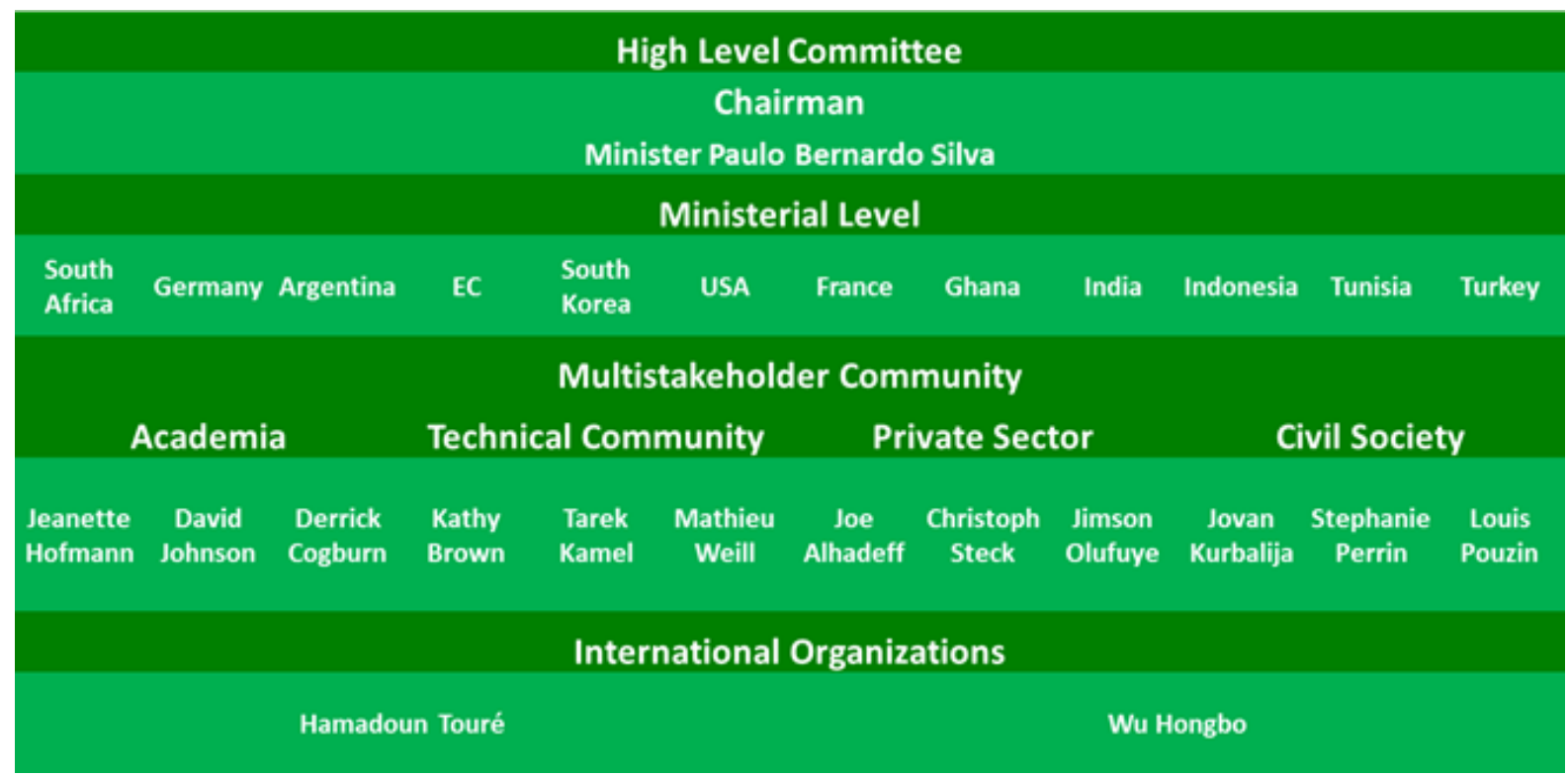

Figure 2. HLMC Composition.

The EMC was composed of: a) eight Brazilian members appointed by CGI.br (one co-chair from the technical community, one representative from academia, and two each from government, civil society and private sector); b) nine representatives from the global multistakeholder community selected through 1net (including one co-chair and one member from the technical community and two each from the private sector, civil society, and the academic community); c) finally, one from the United National Department of Economic and Social Affairs (UN DESA) (appointed by the UN from the staff of the international organizations). After the EMC was formed, a representative from the government of Egypt and a representative of the European Commission were added.

1net was responsible for selecting the global non-governmental members of the EMC and HLMC. This process was coordinated by the 1 net steering committee. Each steering committee member was responsible for coordinating discussions within their constituencies to select individuals for the HLMC and EMC. 


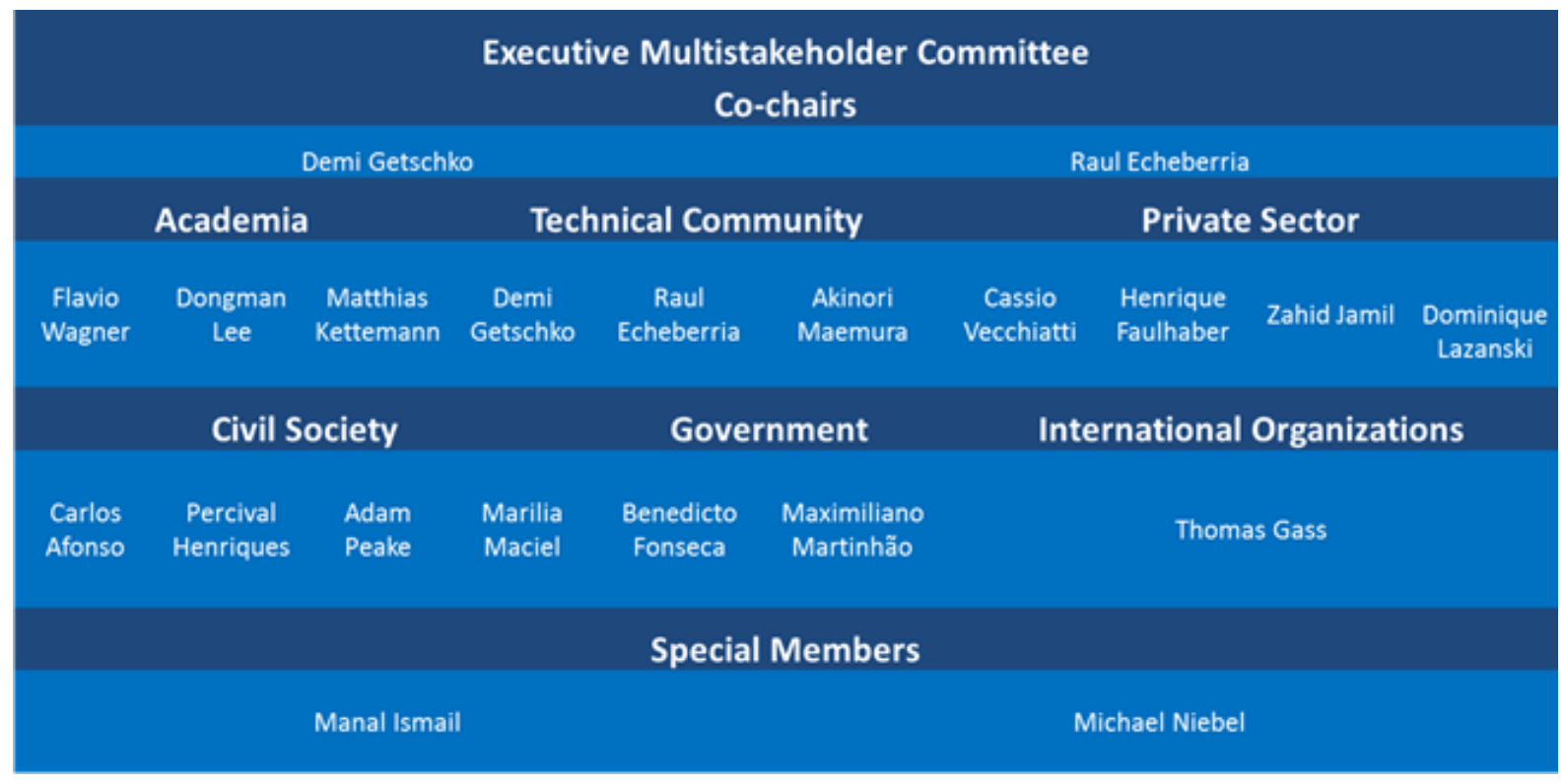

Figure 3. EMC composition.

The Logistics and Organizational Committee was composed of two members from CGI.br (one of them was a co-chair), a member of ICANN (also co-chair), a member of 1net, and three members from the Brazilian government (one from the Ministry of Justice, one from the Ministry of External Relations, and one from the Cabinet of the Presidency).

The Council of Governmental Advisors, which was formed by participating government representatives, was coordinated by the Brazilian Ministry of External Relations and co-chaired by the governments of China, Portugal, and Australia. The mandate of this council was to channel the content proposals and comments coming from government participants. The council had a preparatory meeting on April 22nd in Sao Paulo, one day before NETmundial. During this meeting, government representatives exchanged their impressions about the preparatory process for NETmundial, and also discussed their positions regarding Internet governance principles and the roadmap for the further evolution of the Internet governance ecosystem as proposed in the draft outcome document.

Due to the different mandates and characteristics of each committee, the working procedures varied and were adjusted during the organizational phase. A brief description of the dynamics developed by each group can provide a sense of the decision-making processes. The Executive Multistakeholder Committee (EMC), which had the mandate to determine the overall processes, had the highest number of meetings (eight in total) to prepare proposals related to participation, meeting format, and the production of the first draft of the outcome document. Most meetings occurred in CGI.br's headquarters, but given the composition of the EMC, which included members from all over the world, all meetings included remote participation using Adobe Connect.

EMC decisions were constantly reported to the NETmundial chair by the Secretariat. Board meetings were conducted to review the EMC decisions and provide further input. Such meetings were the ultimate decision-making point and included the chair and co-chairs of NETmundial and the chairs from the EMC and HLMC. Their discussions mostly served to review and endorse 
the approaches adopted by the EMC, and any substantive suggestion from the board was taken to the EMC through its chairs.

The HLMC working process differed from the EMC. One reason for this was the high-level composition of the group and the resulting difficulty it had in gathering its members on a regular basis. A face-to-face meeting occurred during the GSMA forum in Barcelona on February 14th, 2014, since the event counted on the participation of several high-level representatives from the committee.

The second and final face-to-face meeting of the HLMC happened during NETmundial on April 25th, when the outcome document was presented by the EMC for final review before the closing ceremony. Besides those meetings, HLMC members reported having had little guidance from the chairs and little inter-committee communications, which led to a lack of clarity about the role they should play. One HLMC member reported "we have been left without guidance on the process and without work to do, till [sic] the EMC draft outcome arrived." ${ }^{\text {, Th }}$ This helps to explain the limited involvement of this committee during most of the organization process.

The creation of NETmundial committees was important for several reasons. First of all, it strengthened the bottom-up multistakeholder characteristics of the meeting. Secondly, it increased the transparency of the event, by giving stakeholders individuals that they could communicate with in case they had questions or suggestions to make. It also helped to maintain trust, even when procedural aspects of the meeting were not always clear or made known in advance. Finally, the committees became official instances of decision-making authority, which helped to minimize informal and power-based influence on the organization of the meeting.

It is interesting to notice that the composition of the committees endorsed the interpretation that the technical and the academic communities have the same status as other stakeholderssomething that was not well established in the Tunis Agenda. In addition, NETmundial also recognized the academic community as a distinct stakeholder group. This is a positive trend, since academics have been historically identified with the technical community or civil society. These groups have their own common language and strategies to intervene in discussions, which are not always the same ones normally followed by academics. In order to take advantage of academics' expertise, it is important to fully recognize them as a stakeholder group.

In spite of the positive aspects that the committees brought about, the way that they were populated presented some issues. First of all, the procedure that each constituency followed to appoint its representatives has not been made public, although civil society has discussed the selection process in its mailing list and the archives can be retrieved online. ${ }^{33}$ Names were

\footnotetext{
${ }^{32}$ HLMC member, private communication with author, April 11, 2014.

${ }^{33}$ Civil society organized itself into a coordination group, a loose body with the aim to facilitate joint civil society participation in several nominating processes. At the time of NETmundial, it was comprised of representatives from the following civil society coalitions or networks: Internet Governance Caucus, Diplo Foundation, Best Bits, the Non Commercial Stakeholder Group of ICANN, and the Association for Progressive Communications. Among the criteria the coordinating group used to select its representatives, were: 1) Ability to represent civil society as a whole, not just her individual organization; 2) Ability to work collegiately with other stakeholder groups in a multistakeholder setting; 3) Ability to consult widely with civil society groups and to report back as the process progresses; 4) Ability to represent civil society at a senior level in these discussions; 5) Broad knowledge of Internet governance issues and the range of civil society perspectives on these issues; 6) Capacity to participate assertively
} 
forwarded to the organizers of NETmundial through the 1net platform without procedural information about the selection. More transparency about the selection process could have increased the legitimacy of the committees and strengthened the connection between representatives and their stakeholder groups.

The composition of the EMC presented two main issues. Firstly, the group was unbalanced in terms of diversity. Nine of its members were from Brazil and ten were from Latin America. Considering the fact that the importance of the group grew throughout the preparatory process, including drafting the initial outcome document, this unbalance became increasingly relevant. The participation of other governments - only Brazil was an official member of the EMC - could have helped to minimize some of the criticism of the process. In particular, some governmental representatives expressed concern after the final plenary of NETmundial regarding the lack of transparency of the drafting process. According to the representative of the Russian Federation, "all the decisions were made by a specially formed committee and we were unable to understand the principles underlying the shaping of such a committee. ${ }^{34}$

Secondly, the inclusion of special members was not based on any clear justification; they were just added to the mailing list of the EMC. This addition was well accepted by EMC members and did not stir controversy. However, despite the increased regional diversity the special members provided and the high quality contributions they offered, this late inclusion lacked transparency.

Finally, there was lack of clarity and adequate communication between the committees, especially between the HLMC and the EMC while drafting the outcome document. There was also some overlap and duplication among committees, particularly regarding the role of governmental representatives in the HLMC and in the CGA when it came to the tasks of providing political articulation and fostering the involvement of the international community.

\section{Participation and Decision-Making Structures}

In line with the spirit of WSIS and the Tunis Agenda, ${ }^{35}$ the NETmundial process followed principles of openness, inclusiveness, and transparency. It aimed to involve governments, the private sector, civil society, intergovernmental and other international organizations, as well as the academic and technical community. ${ }^{36}$

Two different modalities of participation were used: 1) using online tools on www.netmundial.br for collecting contributions; and 2) participating at the NETmundial meeting.

A group of civil society organizations proposed online consultations to the Brazilian government in a meeting that took place during the IGF Bali (September 2013). The idea drew on the successful experience of the process leading up to the passage of the Brazilian Civil Rights Framework for the Internet (Marco Civil), a law that was drafted collaboratively online based on the inputs from a range of stakeholders. As a follow up to the IGF meeting, Best Bits, a network

and creatively. Jeremey Malcom, e-mail to Best Bits mailing, Dec. 22, 2013,

http://lists.bestbits.net/arc/bestbits/2013-12/msg00272.html.

${ }^{34}$ Statement of the Russian Federation at the NETmundial closing session, April 24, 2014.

${ }^{35}$ See WSIS, "Geneva Declaration of Principles," WSIS-03/Geneva/DOC/4-E, Dec. 12, 2003, ๆ 48, http://www.itu.int/wsis/docs/geneva/official/dop.html; WSIS, "Tunis Agenda for the Information Society," WSIS05/TuNIS/DOC/6 (Rev. 1)-E, Nov. 18, 2005, ๆ 29, http://www.itu.int/wsis/docs2/tunis/off/6rev1.html.

${ }^{36}$ See Tunis Agenda, at 9 ฯ 35-36. 
of civil society organizations, sent a letter to the hosts of NETmundial suggesting concrete mechanisms that would "facilitate purposeful deliberation and help to narrow down the meeting's conclusions." 37 Additionally, online platforms were used for consultation and facilitating deliberation during NETmundial.

The first phase of consultations (February 14 - March 8) consisted of collecting submissions through a dedicated web-form on the two agenda topics. A total of 188 contributions were received: 65 were related to principles, 69 were dedicated to the roadmap, and 54 combined the two topics. The majority of contributions were from United States (31) and Brazil (16), followed by the United Kingdom (7), India (7), and Switzerland (6).

From an inclusiveness and equality standpoint, it should be noted that submissions were only allowed in the English language, due to the limited time available for the analysis of contributions. Although this may have negatively impacted participation, the organizers felt compelled to sacrifice multilingualism at this stage, despite it being one of the guiding principles established by WSIS $^{38}$ and the Tunis Agenda. ${ }^{39}$

All the contributions were made available online, which added to the transparency of the process. Anyone interested could develop an analysis of the inputs. The Diplo Foundation conducted a data mining exercise, for example, ${ }^{40}$ and the Center for Internet and Society in Bangalore offered a data visualization platform. ${ }^{41}$ Since the participants would have only two days of discussions in São Paulo, meeting organizers wanted a draft outcome document in order to give attendees a starting point for discussion. The EMC was directed to produce this first draft based on the inputs provided in the public consultation. ${ }^{42}$ The Secretariat of NETmundial assisted in this process by producing a summary of all the inputs. ${ }^{43}$

To prepare the draft outcome document, the EMC followed a few guidelines. Primarily, they opted to include the issues mentioned in a large number of contributions (relevance) and tried to address them in a way that made consensus possible (consensus-driven approach). In order to be included in the draft, each paragraph needed to achieve consensus among EMC members and also have a reasonable expectation of achieving consensus in the NETmundial plenary. EMC members tried to ensure the document reflected the content of inputs instead of representing the points of view of their own stakeholder groups. They were largely successful in impartially

\footnotetext{
${ }^{37}$ Best Bits, "Submission to Brazil meeting committees on deliberative processes for the Brazil meeting," February4, 2014, http://bestbits.net/brazil-processes/.

${ }^{38}$ See WSIS Declaration, at $948,52$.

${ }^{39}$ See Tunis Agenda, at $₫$ 29, 49, 53.

${ }^{40}$ Vladimir Radunovic, "Predicting NETmundial: What does data-mining the contributions tell us?," Diplo Foundation, April 18, 2014, http://www.diplomacy.edu/blog/predicting-netmundial-what-does-data-miningcontributions-tell-us.

${ }^{41}$ Sumandro Chattapadhyay, "NETmundial- Which Countries Have Not Submitted Contributions to NETmundial?," The Centre for Internet \& Society, April 22, 2014, http://cis-india.org/internet-governance/blog/net-mundial-whichcountries-have-not-contributed-to-net-mundial.

${ }^{42}$ All received contributions can be found at http://content.netmundial.br/docs/contribs.

${ }^{43}$ NETmundial, "Content contribution Summary," March 17, 2014, http://netmundial.br/wpcontent/uploads/2014/05/Content-Contribution-Summary_1703_final.pdf.
} 
representing the inputs, ${ }^{44}$ but there was no clear guideline about that before the drafting work started. It is important to notice, however, that these guidelines were not publicly made available, and were only informally discussed among EMC members. This may have led to a lack of understanding about the criteria for drafting the text and how conflicting views were resolved. According to the representative of the Russian Federation, "we are in the dark as to what principles were used to select which comments to incorporate and which not to incorporate." 45

In order to develop the draft outcome document, the EMC divided itself in two groups: one focused on the drafting of the principles section and the other focused on the roadmap section. One of the reasons for this was the unexpectedly large amount of contributions received during the consultation that needed to be reviewed by the EMC in a short timeframe to produce the draft outcome. Having two drafting groups working in parallel was the only way to make sure that a draft outcome document could be successfully produced in time. Moreover, specialization allowed EMC members to focus on the contributions related to the agenda item they were working on and to do a careful mapping of the different opinions. This mapping made it easier to identify the areas of potential agreement. The final work was reviewed by the whole EMC. The success of this specialization within the committee suggests that relatively small and issueoriented groups may be a good way to structure similar drafting endeavors.

The second phase of online consultation followed the publication of the draft outcome document on the NETmundial website on April 11th. From April 15th to April 21st, the public could comment on the draft text. This phase generated 1370 comments, which were then assembled by the NETmundial data team into a spreadsheet. ${ }^{46}$

A website was specially prepared to receive public comments based on a dedicated system (Wordpress plugin) called "Commentpress," 47 which allows short comments in specific parts of a document. The system requested users to identify themselves and special customizations were made for NETmundial's purposes, such as asking contributors to identify their affiliated stakeholder group. Although there was no mechanism to validate the real identity of each contributor, each comment showed the name openly for further validation. No issues related to misbehavior or manipulations were registered; on the contrary, the availability of such a system was perceived as a good format to initiate the discussion before the meeting took place. The system remains available for review on the event website. ${ }^{48}$

Subsequently, the Secretariat extracted the most meaningful comments (based on the existence of a "rationale" for the suggested edit) ${ }^{49}$ and produced a summary, which was published on April

\footnotetext{
${ }^{44}$ Raúl Echeberría, "IGF 2014 Pre-Event: NETmundial Looking Back, Learning Lessons and Mapping the Road Ahead," video recording,

https://www.youtube.com/watch?v=Iw8xOJL1BoE\&index=188\&list=UUk0zf4oI0IsJLh1owvUQSfQ

${ }^{45}$ Statement of the Russian Federation at the NETmundial closing session. April 24, 2014.

${ }^{46}$ Spreadsheet with all the public inputs is available at: http://netmundial.br/wpcontent/uploads/2014/05/NETmundial-Comments.xlsx.

${ }^{47}$ More information about Commentpress is available at: http://futureofthebook.org/commentpress/

${ }^{48}$ The interface for public comments is available at: http://document.netmundial.br/.

${ }^{49}$ In the words of the Secretariat, "rationale" includes "even the most primary forms of supporting arguments. Some rationale are elaborated and go deep into the subject. Others are one-liners which will not develop the subject as much as other arguments. Nevertheless, despite the stark difference between those simpler rationale and deep arguments that elevate the level of the debate, they are something to start with and that is why they are included in the report." See "NETmundial Draft Outcome Document—Public Consultation: final report on comments," April
} 
22. In the document, the Secretariat acknowledged the imperfection of this consolidation, suggesting that the best and most transparent approach would be to go through all the comments one by one - something that the EMC endeavored to do in the single day remaining prior to the start of the meeting. However, given the time constraints under which the EMC operated, it is not clear whether comments were sufficiently analyzed. The EMC did not produce another version of the outcome document: online comments on the original draft and comments made in person by NETmundial attendees were incorporated simultaneously. If time was sufficient, it would have been useful to produce and publish an intermediary version of the draft outcome document incorporating the results of the online comments prior to the in-person comments.

To manage and accept registrations for on-site participation, the EMC was guided by various principles, such as openness, equality, and flexibility. ${ }^{50}$ In this light, a decision was made to adopt a pre-registration procedure, inviting anyone with an interest in attending the meeting to sign up via a dedicated web form between February 3rd and February 28th. Expressions of interest allowed the organizers and the EMC to monitor the levels of attendance, keep track of the balance among the stakeholders, and develop early strategies to cope with the possibility of over-registration. ${ }^{51}$

On March 15th, participants were informed of the outcome of their requests for participation. Due to interest exceeding available capacity, the EMC used criteria to select among participants. These criteria included: a limit of no more than five people from each organization (up to two from the same organization and also from the same country); up to three governmental representatives per country if the country was represented at a ministerial level and two in case of no ministerial representation. The EMC guaranteed that each country would have at least one representative. $^{52}$ The Brazilian Ministry of External Relations through diplomatic channels handled the registration of governments. ${ }^{53}$

Other general guidelines adopted by the EMC were intended to foster balance among stakeholder groups, giving preference (in the form of pre-registration) to those that showed previous experience with the theme and prioritizing the registrations of actors from underrepresented countries. Eventually, the total number of registered participants was 933, with 364 governmental representatives, 87 coming from academia, 160 from civil society, 141 from the private sector, 109 from the technical community, 186 from the press, and 51 from other

22, 2014, p. 8 (emphasis in the original), http://netmundial.br/wp-

content/uploads/2014/04/NETmundialPublicConsultation-FinalReport20140421.pdf.

${ }^{50}$ EMC, "First Meeting."

${ }^{51}$ A total of 869 expressions of interest were received coming from 94 different countries, divided into self-declared categories of academia (105), civil society (245), government (138), private sector (210), technical community (107) and "other" (64). The highest numbers of requests were, much like in the case of submissions, from Brazil (251) and the United States (136).

${ }^{52}$ EMC, "Executive Summary: Teleconference Meeting," March 10, 2014, http://netmundial.br/wpcontent/uploads/2014/01/EMC-meeting-report_1003_final.pdf.

${ }^{53}$ This explains why there were 138 expressions of interest from governments and 364 governmental representatives: not all of the government representatives filled out the web form. 
categories (41 of which were classified by the Secretariat as from "international organizations"). ${ }^{54}$

The management of funding for the organizational aspects of NETmundial fell under the mandate of the LOC, who sought resources from CGI.br, ICANN, and the I* community. One fundamental question for the LOC was whether any travel support would be available. From an early stage, it was decided that the meeting would be similar to the Internet Governance Forum, with no funding officially provided by the organizers, but some funding would be offered by organizations that usually support Internet governance-related meetings. However, the LOC sought funding from those various Internet-related institutions with no success. Finally, at the beginning of April, approximately 3 weeks before the meeting, the Secretariat instituted a donor fund to support people who requested it. However, since participants who submitted requests were not informed until very late in the process, this prompted many of them to seek other sources of funding or abandon the idea of attending altogether.

Participation during the meeting was also made possible through online interaction. This was done in two different ways. First, participants could submit chat comments to the online moderator via Adobe Connect. This would then result in the question being read out loud by the moderator. Second, individuals could participate remotely via one of the 33 hubs available worldwide, spread across 30 cities and 23 countries. This distribution was the result of an open "call for remote hubs proposals" issued on March 18. ${ }^{55}$ Ten of the 33 remote hubs were given the status of official hub on the basis of geographical coverage, adequacy of infrastructure, personnel provision, and costs. In these official hubs, connection would be possible via video, as opposed to merely by text. Moreover, the crucial difference between accessing the event at an official hub as opposed to Adobe Connect was that participants could benefit from simultaneous translation of the meeting into six languages (Arabic, Chinese, French, Portuguese, Russian and Spanish) in addition to English, and have their comments translated into English from one of these languages. Remote participation was a key element to broaden the scope of participants in NETmundial and to enhance transparency.

One of the most innovative features of the NETmundial meeting concerned the management of microphones for comments from the audience and from remote participants: four different microphones were provided for comments from each stakeholder group, and the opportunity to speak rotated to each microphone. The four microphones were allocated to: 1) civil society; 2) the business community; 3) the technical and academic communities; and 4) government and international organizations. Thus, equality among different stakeholder groups was ensured by having participants from each of these groups queue in different lines in order to make their comments. Each commenter was allotted the same time (a maximum of 2 minutes). In addition, on each rotation inputs would be gathered from remote participants - so there was a fifth microphone in practical terms-applying the same time limit. Thus, government

\footnotetext{
${ }^{54}$ Chairman Virgilio Almeida provided the most recent participation data in the article "The Evolution of Internet Governance: Lessons Learned from NETmundial," Internet Computing, IEEE, Sept.-Oct. 2014, http://ieeexplore.ieee.org/xpl/articleDetails.jsp?arnumber $=6886132$.

55 "NETmundial opens opportunity for interested to host Official Hubs of participation," NETmundial, March 18, 2014, http://netmundial.br/blog/2014/03/18/netmundial-opens-opportunity-for-interested-to-host-official-hubs-ofparticipation/.
} 
representatives - eveb those on the ministerial level - stood in line waiting for their time to speak.

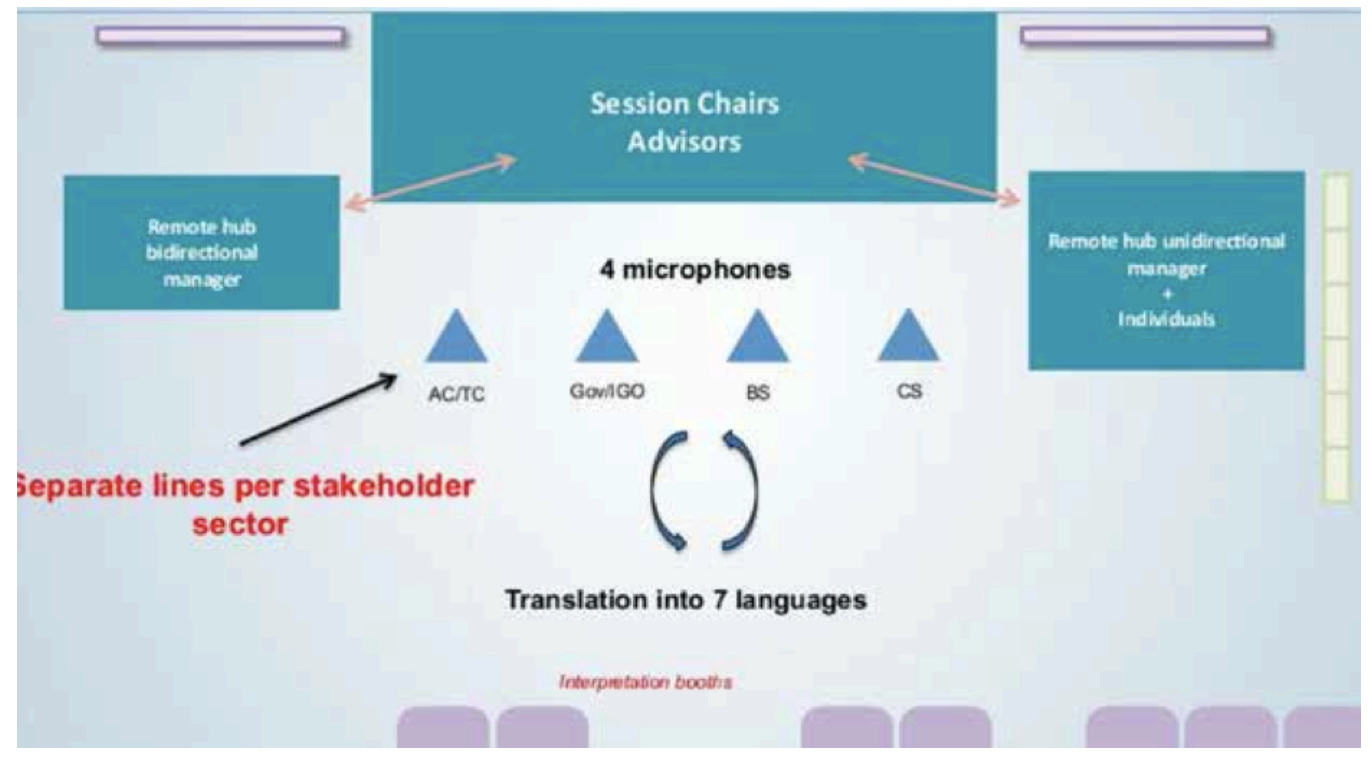

Figure 4. NETmundial meeting structure.

The meeting started with an opening ceremony followed by welcoming remarks. Although these sessions were important for encouraging the participation of governments, they delayed the multistakeholder working sessions. The working sessions of the meeting revolved around the two agenda items. They were conducted by individuals that were invited to be the chairs of the working sessions, which should not be confused with the co-chairs of the meeting. ${ }^{56}$

The sessions of the first day, entitled "Principles Part I" and "Roadmap Part I," gave rise to a lively debate. After the working day finished, drafting groups met in a separate room. Two separate groups were created, one for principles and another for the roadmap, similar to the structure the EMC used for preparing the draft outcome document. These groups were composed of the chairs of the working sessions and the members of the EMC (also called "advisors" in the document that announced the dynamics for the working sessions). ${ }^{57}$ The groups made changes in the outcome document in order to reflect comments made in the online consultation and in the plenary sessions. The same process was followed at the end of the second day for the elaboration of the final text, after incorporating into the outcome document the comments made in working sessions III (entitled "Principles Part II") and IV (entitled "Roadmap Part II").

The drafting room was open to observers and the text was displayed on the screen of the conference room along with the modifications as they were made, allowing for transparency in the drafting process. Although the procedures for the drafting session explicitly prohibited any interference from observers, this prohibition was not strictly enforced and as a result several

\footnotetext{
${ }^{56}$ The chairs of the working sessions on principles were Markus Kummer and Adiel Akplogan, and the chairs of the working sessions on the roadmap were Janis Karklins and Jeanette Hofmann.

57 "NETmundial announces dynamics for working sessions," NETmundial, April 20, 2014, http://netmundial.br/blog/2014/04/20/netmundial-announces-dynamics-for-working-sessions/.
} 
observers engaged in bilateral conversations with members of the drafting committee, influencing the drafting process.

Since it was not clear from the outset whether this form of lobbying would be permitted, some stakeholders were not in the drafting room. This put them at a disadvantage compared to the more organized set of participants (especially from governments and the business constituency) who were present and able to more consistently intervene in the process. ${ }^{58}$ Furthermore, the lack of a sufficient number of trusted experts among the advisors to the chairs appears to be a weak point of the drafting process. In the roadmap drafting group, this lack was particularly felt when dealing with mass surveillance and privacy. While the source of the legitimacy for the drafting group was political - their constituencies chose them - the legitimacy of the assistants was based on expertise.

Finally, some controversy arose about the involvement of the HLMC in the drafting of the outcome document. This happened in two moments. When the first draft was produced by the EMC, the HLMC played a role in reviewing it before it went public. Some HLMC members presented comments individually, but there was no joint committee discussion about the draft. This lack of common position made it harder for the EMC to clearly assess if suggestions from individual HLMC members garnered enough support to be incorporated into the draft.

The second moment happened during a meeting to present the outcome document to the HLMC that was arranged right before the final plenary session. The organizers of NETmundial tried to express deference to the committee and probably expected the meeting to be a formality. Nevertheless, the HLMC decided to propose some substantive modifications to the text. Tension in the room rose and the meeting was close to a breaking point. Some HLMC members took advantage of the moment to advocate the inclusion of their own positions in the text (see section E on outcomes) and a few changes were accepted to save the outcome document. This meeting of the HLMC added an element of top-down decision making into the bottom-up process.

\section{Outcomes}

\section{A. Political Outcomes of the Meeting}

According to most analyses "the most remarkable aspect of NETmundial was its process."59 NETmundial will be positively remembered for having achieved a bottom-up multistakeholder outcome document. The meeting triggered positive reactions throughout the Internet Governance ecosystem. During the IGF open consultations that took place in Paris (May 1-2 2014), there was overwhelming recognition of the relevance of NETmundial as a learning opportunity. NETmundial was also one of the main topics discussed in the IGF 2014, in Istanbul (September 1-5), with several workshop sessions and a pre-event dedicated to it.

Nevertheless, this positive understanding is not unanimous. During the closing session Russia, India, and Cuba expressed their reservations regarding the process and its outcome document. ${ }^{60}$

\footnotetext{
${ }^{58}$ Gabrielle Guillemin, “Netmundial: Success or Failure?,” Article 19, April 29, 2014, http://www.article19.org/jointhe-debate.php/143/view/.

${ }^{59} I d$.

${ }^{60}$ See Transcripts of NETmundial closing session, April 24, 2014, http://netmundial.br/wpcontent/uploads/2014/04/NETMundial-23April2014-Closing-Session-en.pdf.
} 
This public divergence seems to have driven further apart countries that pursued alliances in the past, such as India and Brazil, ${ }^{61}$ and showed some of the difficulties in articulating political alliances among the BRICS nations (comprised of Brazil, Russia, India, China, and South Africa).

\section{B. Outcome Document}

On a more substantive level, NETmundial's output document represents several achievements. The outcome document does not have a binding nature, but it has become an important reference point for global discussions due to the multistakeholder approach used for its development. It placed human rights as the cornerstone of the Internet governance ecosystem, it reinforced the commitment to strengthen the Internet Governance Forum, it managed to cover topics that were considered sensitive and were initially not part of the agenda (such as mass surveillance), it supported distributed mechanisms of governance, it emphasized the importance of enhancing both democratic and multistakeholder participation, and it ultimately "represents substantial progress towards public interest-driven Internet governance. ${ }^{.62}$

In spite of the importance of the Multistakeholder Statement of São Paulo, there were several issues that were perceived as shortcomings in the outcome document. It is important to highlight them because there seems to be some misunderstandings about the rationale underlying the treatment of certain topics. This lack of clarity may indicate procedural imperfections in the drafting process, which made it difficult to achieve enough transparency and strike the appropriate balance between competing interests.

1. Net neutrality: in the drafts of the outcome document there is reference to "equal treatment of all protocols and data" and also to "recognizing technical management principles for efficient and improved network operation" and that Internet governance should encourage "the free flow of data packets/information." These references were removed. In turn, a bullet point on the final NETmundial Multistakeholder Statement merely recognizes the need to "continue the discussion of the Open Internet including how to enable freedom of expression, competition, consumer choice, meaningful transparency and appropriate network management and recommend that this be addressed at forums such as the next IGF."

\footnotetext{
${ }^{61}$ During WSIS, the most promising diplomatic alliance for Brazil eventually seemed to be coordination with India and South Africa, two large democratic developing nations. The three members of the IBSA Forum had been working together on Internet governance, interconnection costs, and Intellectual property since the first phase of WSIS, following a proposal from South Africa. However, the IBSA framework agreement for Cooperation on the Information Society would only be adopted in 2006. In 2011 the Brazilian Ministry of External Relations sought support from CGI.br and civil society organizations to organize a multistakeholder seminar. The event discussed substantive issues deemed relevant for developing countries, such as access to infrastructure, commerce and services and institutional frameworks. At the end of the seminar, the government representatives of the three countries drafted a document focused on Internet Governance institutional arrangements. The document with recommendations was supposed to be improved until the IBSA meeting in Durban, but the Declaration of Tshwane only took of the recommendations; the document became a historical reference, not an official IBSA position. The conversations about this topic in IBSA went cold. In fact, the IBSA Forum in general is going through a period of low diplomatic activity.

62 "Association for Progressive Communications (APC) Statement on NETmundial," APC, April 2014, http://www.apc.org/en/pubs/association-progressive-communications-apc-stateme.
} 
2. Intermediary liability: while there was no specific provision on intermediary liability in the draft outcome document, the new text included the principle that "Intermediary liability limitations should be implemented in a way that respects and promotes economic growth, innovation, creativity and the free flow of information. In this regard, cooperation among all stakeholders should be encouraged to address and deter illegal activity, consistent with fair process." This formulation is problematic in the eyes of civil society because the focus on economic aspects prevails over the protection of human rights. Furthermore, the second sentence of the text contains a provision which recalls the OECD's controversial language of voluntary measures to deter infringement in accordance with "fair process" 33 -as opposed to the more stringent right to "due process"-which forms an integral part of human rights jurisprudence.

3. Right to access, share, create and distribute information on the Internet: this right was qualified with the expression "consistent with the rights of authors and creators as established in law," which allows the legislator to create exceptions to the aforementioned rights without explicitly requiring the respect of the conditions identified under human rights law, including necessity and proportionality for the attainment of a legitimate objective.

4. Reference to "necessary and proportionate" in relation to the conditions under which mass surveillance may be permitted: this point was not included in the original draft document, but was included during drafting after having been repeatedly supported at the public comment period and at the meeting. ${ }^{64}$ However, the final text has no mention of this particular limitation.

5. Structural separation of IANA functions and ICANN policy process: the issue of separation was another point that was not included in the original draft document, but was raised by a large number of actors during the meeting and was included in the final draft that was submitted to the HLMC. ${ }^{65}$ The paragraph that was approved followed a last-minute suggestion by ICANN, presented during the controversial HLMC meeting that occurred just before the plenary session. The final text features diluted language, according to which states "it is desirable to discuss the adequate relation between the policy and operational aspects."

\footnotetext{
${ }^{63}$ See OECD, "Communiqué on Principles for Internet Policy-Making," The Internet Economy: Generating Innovation and Growth, http://www.oecd.org/internet/innovation/48289796.pdf. Civil society did not endorse the Communique in light of the vague terminology and the risk that this would lead to censorship. For an account of the civil society reaction, see Milton Mueller, "Civil Society Defects from OECD Internet policy principles", Internet Governance Project, June 28, 2011, http://www.internetgovernance.org/2011/06/28/civil-society-defects-from-oecdinternet-policy-principles/.

${ }^{64}$ The Secretariat of NETmundial produced a second version of the outcome document which tried to reflect the comments received. It was treated as a supporting document to the EMC. This version explicitly mentioned "necessary and proportional." During the drafting exercise, the same expression was included but it got removed after some members of the drafting group withdrew their support. This swing of opinion happened after conversations with some observers, which is evidence of the efficacy of lobbying during the drafting exercise.

${ }^{65}$ For more information about the separation of IANA functions, see Milton Mueller and Brenden Kuerbis, "Roadmap for globalizing IANA: four principles and a proposal for reform," Internet Governance Project, 2014, http://www.internetgovernance.org/wordpress/wp-content/uploads/ICANNreformglobalizingIANAfinal.pdf.
} 
Finally, some of the most important and controversial issues on the agenda remain open after NETmundial, such as the roles and responsibilities of different stakeholders. Remanding the exact definition of these processes to another forum is a consequence of the fact that NETmundial was ill equipped (and never intended) to address highly detailed and complex questions. A similar argument, stemming not only from the technical but also from the political and economic complexity (due to the difficulty of reconciling opposing positions) can be made for other important issues contained in the non-exhaustive list for further discussion, such as jurisdiction on the Internet, net neutrality, and mechanisms of oversight or benchmarking of compliance with the principles: these problems call for complex assessments which make rough consensus more problematic, and therefore warrant separate treatment.

\section{Best Practices and Templates}

Most of the opinions expressed after NETmundial assert that its open process is a key legacy of the event. For many years, the production of outcome documents prepared in a multistakeholder manner with the involvement of a large number of global actors was deemed infeasible. It is important, therefore, to identify positive aspects and areas for improvements of this unique experiment, particularly with respect to transparency, inclusiveness, and accountability.

The list below is an attempt to summarize some lessons that can be taken from NETmundial, which were discussed in more detail in the previous sections of this case study.

Structured multistakeholder dialogue was key to building trust. After the Snowden revelations, trust among actors declined. "Fragmentation of the Internet [was] a very real risk," according to some analysis at that time. ${ }^{66}$ Instead of contributing to the distrust, Brazil came up with an approach for discussing topics in a collaborative fashion. In a moment of crisis, energies were channeled to a clear, common, and positive goal.

It was possible to produce bottom-up, multistakeholder outcomes in a global environment. For many years the production of outcome documents prepared in a multistakeholder manner with the involvement of a large number of global actors was deemed infeasible. NETmundial used an open process of consultations followed by drafting groups to effectively produce an outcome text.

Multistakeholder committees were an important tool for strengthening the bottom-up nature of NETmundial. The establishment of multistakeholder committees in charge of specific aspects of the meeting helped to channel concerns and opinions. Nevertheless, stakeholder groups were inconsistent in how they chose representatives for these committees. This point is raised by the NETmundial Multistakeholder Statement, which mentions that, "Stakeholder representatives appointed to multistakeholder Internet governance processes should be selected through open, democratic, and transparent processes. Different stakeholder groups should selfmanage their processes based on inclusive, publicly known, well defined and accountable mechanisms."

\footnotetext{
66 "Internet Society Urges President Obama to Effect Immediate and Meaningful Changes to U.S. Government Surveillance Practices," Internet Society, January 15, 2014, http://www.internetsociety.org/news/internet-societyurges-president-obama-effect- $\% \mathrm{E} 2 \% 80 \% 98$ immediate-and-meaningful $\% \mathrm{E} 2 \% 80 \% 99$-changes-us-government.
} 
Lack of clarity on decision-making guidelines and drafting reduced accountability. The set of guidelines that were used by the EMC and the drafting groups to choose the topics and positions that were included in the outcome document should have been publicized prior to the public consultation. Moreover, there was insufficient clarity about whether the members of the drafting group represented the interests of their stakeholders or were limited to incorporating the content of public contributions. During the intermediary stages of the drafting, at least succinct reasons for choosing not to follow a particular set of suggestions could have been provided. At NETmundial, this was neither done in the process leading to the first draft of the outcome document, nor in the phase that took into account the comments made on that first draft. Repudiation of the final document by the representative from Russia, and the dissatisfaction of India and various nongovernmental stakeholders with the way in which inputs were fed into the text illustrated that the lack of clear criteria generates a sense of alienation from the process.

Flexibility in the procedures was crucial to the achievement of results within a limited time frame, but sufficient time for the assessment of contributions is essential to further democratic participation. Procedures followed by the NETmundial committees were largely inspired by the principle of flexibility, which made particular sense in light of the very limited time-frame. Nevertheless, flexibility should never be seen as a justification for failure to respect essential democratic principles, such as allocating sufficient time to choose committee representatives and justifying why some proposals were not included in the draft outcome document. In addition, the process could better respect the fact that stakeholders often have different needs when it comes to the time needed to develop a response. For example, governments have to follow an internal hierarchy before they are able to present an official contribution in a public consultation period. Moreover, depending on the content of the outcome document, they may also need to consult with their principals before endorsing it. Developing procedures that take into account these differences is key to promoting equal participation.

The specialization of tasks and the assistance of experts facilitated the drafting exercise. While drafting the first version of the outcome document, the EMC divided itself in two groups, one devoted to principles and other devoted to the roadmap. The final work was reviewed by the whole EMC. This division into relatively small and issue-oriented groups was a good way to structure the drafting process. In addition, the use of trusted experts to assist the drafting group on technical or other specific issues was helpful. While the source of the legitimacy of the drafting group was political-their constituencies should choose them-the legitimacy of the assistants was based on expertise.

Concrete management of the process and the setup of the meeting had an impact on equality among participants. As noted, an innovation of the NETmundial meeting was the creation of different microphone queues for each stakeholder group and for remote participants. This put every stakeholder in a situation of formal equality during the plenary sessions. In other stages of the process, however, this equality was maintained to a lesser degree. For instance, the involvement of certain governments through the HLMC (where governments had more seats than any other constituency) afforded them the opportunity to intervene with more weight in the last stage of negotiation of the outcome document. This meeting of the HLMC added an element of top-down decision making into the bottom-up process.

On-site participation was enriched and complemented by online consultations. NETmundial used online channels for enhancing openness and participation in the process of shaping the 
outcome document. Open-ended consultations, in which participants were able to contribute their ideas freely was combined with platforms that allowed adding comments to specific provisions of the text.

Remote participation mechanisms was key to fostering wider participation. NETmundial provided channels for the online participation of individuals and also encouraged the creation of hubs, where several people gathered together to follow the webcast of NETmundial and interact with the meeting. Remote participants were given similar opportunities for participation as onsite participants. In practical terms remote participation represented a fifth microphone with the same time limit as the other four onsite microphones.

More could have been done to improve multilingual participation. Multistakeholder participation ought to promote substantive, instead of merely formal equality. While the organization of NETmundial accommodated the needs of the speakers of seven different languages, it should be noted that the online preparatory phase, including the original submissions and the comments on the proposed text, was only possible in English. This may have led to underrepresentation of non-English-speakers and insufficient discussion of the topics that were of relevance to them. If the number of times an issue was raised was considered criteria for its inclusion in the outcome document, lack of linguistic diversity may have affected the outcome document. 


\section{Appendix K: \\ Swiss ComCom FTTH Roundtable Case Study}




\title{
NoC Internet Governance Case Studies Series: Swiss ComCom FTTH Roundtable Case Study
}

\author{
Urs Gasser, Ryan Budish, David R. O’Brien \\ Sarah Myers West, Sergio Alves Jr., and Alexander Sculthorpe \\ Berkman Center for Internet \& Society at Harvard University
}

Editorial Note: Context, Character, and Purpose of the Case Study

This case study is part of a globally coordinated, independent academic research pilot project by the Global Network of Interdisciplinary Internet \& Society Research Centers (NoC). Facilitated by the Berkman Center for Internet \& Society at Harvard University, this study examines existing multistakeholder governance groups with the goal of informing the future evolution of the Internet governance ecosystem. Building upon the NETmundial Principles and Roadmap, it contributes to current policy debates at the international level, including the Internet Governance Forum, the NETmundial Initiative, and other organizations and efforts.

Internet governance is an increasingly complex concept that operates at multiple levels and in different dimensions, making it necessary to have a better understanding of both how multistakeholder governance groups operate and how they best achieve their goals. With this need in mind, at a point where the future of Internet governance is being re-envisioned, colleagues from several NoC institutions around the world have written twelve case studies examining a geographically and topically diverse set of local, national, and international governance models, components, and mechanisms from within and outside of the sphere of Internet governance. Key findings from these cases are summarized in a synthesis paper, which aims to deepen our understanding of the formation, operation, and critical success factors of governance groups and even challenge conventional thinking.

The research, based on twelve case studies, suggests that there is no single best-fit model for multistakeholder governance groups that can be applied in all situations. Rather, it reveals a range of approaches, mechanisms, and tools available for both the formation and operation of such groups. The analysis demonstrates that the success of governance groups depends to a large degree on the careful selection, deployment, and management of suitable instruments from this "toolbox." As governance groups pass through different phases of operation, conveners and facilitators must remain alert to changes in circumstances that necessitate adjustments to the approaches, mechanisms, and tools that they deploy in order to address evolving challenges from inside and outside. This case study provides insights into how those instruments can be deployed and adjusted over time within such groups, and highlights how their interactions with important contextual factors may be successfully managed within given resource restraints.

The research effort is grounded in a diversity of global perspectives and collaborative research techniques. Adhering to objective and independent academic standards, it aspires to be useful, actionable, and timely for policymakers and stakeholders. More broadly, the Network of Centers seeks to contribute to a more generalized vision and longer-term strategy for academia regarding its roles in research, facilitation and convening, and education in and communication about the Internet age.

For additional information on the initiative, please contact Urs Gasser, Berkman Center for Internet \& Society, at ugasser@cyber.law.harvard.edu. 


\begin{abstract}
This case study explores the dimensions of how the Swiss government-in particular, the Swiss Federal Communications Commission (ComCom) and the Swiss Federal Office of Communications (OFCOM) - used a multistakeholder process to organize private sector firms to begin deploying in a coordinated fashion a fiber optic network connected to every home in Switzerland. Although fiber optic connections were available in some areas of Switzerland, telecommunications and cable companies in many regions of the country had not yet upgraded older network infrastructure to fiber or connected it to homes. The large capital investments required, competing interests among stakeholders, and the lack of coordinated efforts to serve common interests were all contributing factors. The Swiss government was eager to see the national infrastructure upgraded, but lacked the formal authority to regulate the fiber optic networks under its communications laws. In lieu of regulation, the Swiss government convened a series of voluntary roundtable meetings between 20082012 to achieve a consensus-based solution. The Roundtables, and related technical working groups, attempted to bring together the critical stakeholders and surface the key issues that needed to be resolved. Using Swiss public records, reports, and personal accounts of those directly involved, this case study examines the roles and approaches used in the fiber to the home (FTTH) Roundtables and evaluates the process used as a model of ad hoc, problem solving and distributed governance.
\end{abstract}




\section{Table of Contents}

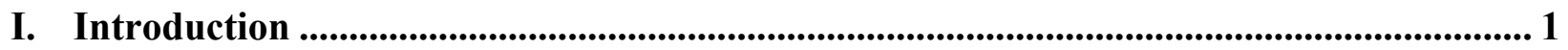

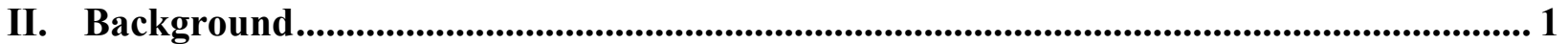

A. Limited Competition Pre-2007 ……................................................................................................. 1

B. Market Disruption in 2007 .................................................................................................................. 3

III. FTTH Roundtables: Improving Coordination Among Stakeholders................................ 4

A. Why ComCom? ................................................................................................................................... 4

B. The Goals of the Roundtables...................................................................................................6 6

C. Beginning the Roundtables.............................................................................................................. 6

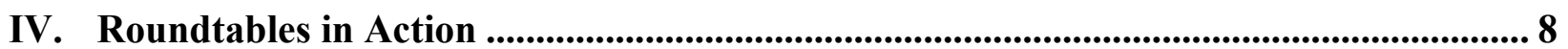

A. The Structure of the Roundtables .......................................................................................... 8

B. The Roundtable Meetings .................................................................................................... 8

C. Confidentiality ……...................................................................................................... 9

D. ComCom as Mediator? ............................................................................................................................. 9

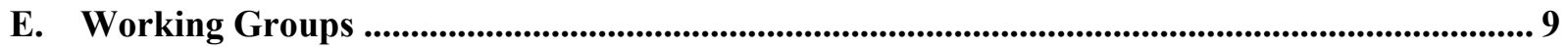

V. Decision Making and Outcomes................................................................................ 10

A. Use of Consensus....................................................................................................................................... 10

B. Defining Success ................................................................................................................... 11

VI. Observations on Particular Features .................................................................................... 12

A. Regulation May Not Be Necessary to Compel Participation Where Market Incentives are

Sufficiently Strong ....................................................................................................................................... 13

B. Participants May View the Value of Transparency Differently ................................................. 13

C. Reducing Rules or Procedures Comes With a Tradeoff............................................................ 13

D. Separation of Strategic and Technical Domains May Be an Effective Approach to Addressing Complex Issues ................................................................................................................... 13

E. Skilled Facilitation May Address Issues of Incomplete Representation .................................... 13

F. Ad Hoc Groups May Be Built on Already Established Structures............................................. 14

G. Subject Knowledge May Be an Important Strategic Resource ................................................... 14

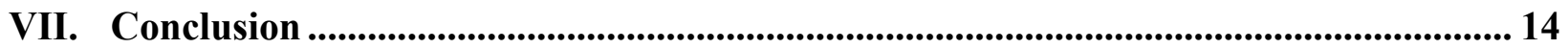




\section{Introduction}

In 2008, Switzerland faced a difficult question. High-speed Internet technologies were quickly evolving, but few homes were connected to the next-generation fiber optic technologies and Switzerland's fiber penetration rate was falling behind that of other countries. At the heart of the issue was how to deploy "fiber to the home" (FTTH) - the last portion of fiber optic cabling necessary to connect homes and buildings to the existing fiber optic network infrastructure. FTTH connections would offer substantial improvement in speed and capacity, paving the way for new, innovative web services in the future. Few regions in Switzerland had FTTH, and deploying it would be a costly investment for private-sector companies, which owned and operated the existing older-generation copper wire connections and had already invested in new technologies that would provide incremental speed improvements in the connections. A number of stakeholders at all levels, including established incumbents, new market entrants, property owners, and others, were also interested in how FTTH deployment was to proceed.

Coordination in Switzerland, however, was complicated by the fact that no government agency had the regulatory authority to compel stakeholders to cooperate or to mandate that they invest in FTTH. Despite that lack of formal authority, it seems that a mix of soft-power and skilled facilitation was able to elicit cooperation among this diverse array of stakeholders and ultimately enable the expansion of FTTH in Switzerland. The effort was led by the Swiss Communications Commission (ComCom), which convened a series ad hoc, distributed, multistakeholder Roundtables over a period of several years. The goal, from the government's perspective, was to convince stakeholders to make the necessary investments, identify and address issues among and between the stakeholders, and to set the stage for an organized deployment of FTTH in a manner that would maximize its economic benefit while minimizing its disruptive effects.

Using public records, written accounts, and interviews with those directly involved, 1 this case study explores the ways in which these Roundtables operated and identifies some of factors that enabled the roundtables to achieve consensus among its participants in the absence of formal regulatory enforcement authority. The case study begins with a discussion of the background factors that led to the Roundtables, including the telecommunications market in Switzerland and its key actors. The case study then describes the formation and operation of the ComCom Roundtables, and finally assesses some of the strengths and weaknesses of the Roundtables as a means of coordinating the actions of diverse, competing stakeholders.

\section{Background}

The events that unfolded in the years prior to the Roundtables are useful for understanding the interests of and challenges facing many of the stakeholders. These years are characterized by shifting power structures within the Swiss high-speed internet industry, new market entrants, changes in technology, regulation or lack thereof, and a desire by government actors for Switzerland to lead in penetration and service.

\section{A. Limited Competition Pre-2007}

Switzerland's telecommunications market is dominated by Swisscom, which was the successor to the formerly state-owned monopoly PTT (Post, Telegraph, Telephone). Switzerland began to

\footnotetext{
${ }^{1}$ Due to the small number of people involved in the roundtables, and our small sample of interviewees, we have elected to keep their identities and specific roles private.
} 
transition PTT to a private company beginning in 1997. Today, Swisscom still has vestiges of state control. The Swiss Confederation owns $51.2 \%$ percent of the share capital and current law ${ }^{2}$ limits private ownership in Swisscom to $49.9 \%$. Its history as a state-owned monopoly placed Swisscom in a privileged position within the market; Swisscom holds a dominant market position in many telecommunications services in Switzerland with $55.3 \%$ of market share in broadband and $62 \%$ in wireless. ${ }^{3}$ By 2012 Swisscom claimed to have the lead in digital TV subscriptions, despite having only entered the market in $2007 .^{4}$

Prior to 2007, Swisscom faced limited threats to its dominance in the telecommunications industry. Although other telecommunications companies were available in the market, they were not significant competitors in several service categories. In the broadband market in particular, its competitors were reliant on Swisscom reselling access to its own network. As ComCom noted in 2007, "in the absence of effective unbundling in the ADSL market, the alternative providers who are still unable to offer anything other than Swisscom's resale products cannot compete against the historic operator and are falling inevitably - and worryingly-behind." 5 Accordingly, as of 2007, Swisscom faced little pressure from other telecommunications providers to begin a costly build-out of fiber optic cable.

Swisscom similarly had little threat in 2007 from cable operators. Although Cablecom, Switzerland's primary cable services provider, has been called "Swisscom's main competitor,", it appears to have been a fairly week one. As of 2003, cable and DSL broadband were effectively equals in the market. However, by 2007, "Swisscom's market share (50\%) [was] more than twice that of Cablecom (approximately 20\%)," leading ComCom to state that "the cable operators appear to exercise insufficient pressure on the broadband market." " Thus, as of 2007 Swisscom faced insufficient competitive pressure to justify an expensive infrastructure investment.

Swisscom was investing in its infrastructure, but not at the level necessary to support FTTH development. As one of our interviewees explained, the traditional model of telecommunication infrastructure improvement reinvests profits from services into incremental growth of the existing network infrastructure; because consumer demand tends to grow slowly, so too does the investment. One interviewee explained that Swisscom was making investments in fiber optics for large businesses, office blocks, and long-distance communication. Additionally, Swisscom deployed fiber optic cables to neighborhoods, reaching as far as street cabinets. But bringing cables "the last mile" into individual homes would have been a difficult and expensive undertaking. According to one of our interviewees, the uncertainty regarding the immediate demand for so much bandwidth and the ability to quickly recover the investment made aggressive FTTH deployment unattractive for telecommunications companies to make solely

\footnotetext{
${ }^{2}$ Swisscom, "Ownership Structure-who does Swisscom belong to?," http://www.swisscom.ch/en/about/investors/shares/ownership-structure.html.

${ }^{3}$ Yochai Benkler, "Next Generation Connectivity: A review of broadband Internet transitions and policy from around the world," Berkman Center for Internet \& Society at Harvard University, (February 2010), pp. 317, 324, ${ }^{4}$ Swisscom, "History: More than 160 years with a finger on the pulse,"

http://www.swisscom.ch/en/about/company/history.html.

${ }^{5}$ Swiss Federal Communications Commission, "Swiss ComCom’s Annual Report 2007,” (2007), p. 10.

6 "Next Generation Connectivity," p. 315.

7 “Swiss ComCom’s Annual Report 2007," p. 10.
} 
based on revenue from existing services. Swisscom seemed content to simply pursue the steady technical improvement of their copper-wire based legacy technology.

\section{B. Market Disruption in 2007}

Beginning in 2007, the market was disrupted by three changes that spurred a rapid growth of FTTH in Switzerland. These disruptions were: (1) legislation opening up access to Swisscom's infrastructure, (2) the entry of the utility companies into the market, and (3) the cable providers adopting the DOCSIS 3.0 standard. Together these changes created an environment where the Roundtables became necessary.

The first of these changes was implementation of the revised Law on Telecommunications. ${ }^{8}$ This legislation included "local loop unbundling" and "subloop unbundling." Collectively, this meant that Swisscom would have to offer competitors access to its last-mile infrastructure at reasonable rates. Writing about this unbundling in 2007, ComCom stated "only with access to this copper cable will alternative providers be able to compete with Swisscom's VDSL offerings and other competitive high-bandwidth products in the future." Although in hindsight unbundling appears to have been insufficient at generating VDSL competition, ${ }^{10}$ in 2007 Swisscom perceived unbundling to be a potential threat to its market position.

The second, and perhaps most significant, market change was the entry of the utility companies into the telecommunications market. Unlike telecommunications companies, Swiss utility companies were in a strong position to invest in FTTH. First, they had the financial resources to support the investment. Many utility companies in Switzerland are publicly held local monopolies that serve a particular geographic area. One of our interviewees described how this arrangement of local monopolies on water, gas and electricity allowed the utilities to marshal the financial resources for a major infrastructure project such as an FTTH deployment. Second, the utility companies needed a high-quality communications network in order to manage "smart meters" located in customer homes. Thus, the utilities needed to make substantial investments in communications infrastructure. Finally, the utilities already owned a series of conduits into homes - water lines, electrical wiring, gar lines, and sewers - and had space in those conduits for fiber cabling.

For all of these reasons, the local utilities began to consider building FTTH and entering the telecommunications market. In 2007, the local utility of Zurich was among the first to announce it would spend CHF 200 million, approximately USD 170 million, to deploy FTTH. Other utilities followed shortly thereafter with similar announcements. ${ }^{11}$ According to one interviewee,

\footnotetext{
8 “Swiss ComCom's Annual Report 2007,” p. 5.

${ }^{9}$ Ibid.

${ }^{10}$ Swiss Federal Communications Commission, “Activity Report ComCom 2012,” (2012), p. 14 (“[T]his decline [in unbundled lines] has to be seen in relation to the development of digital television on the fixed network. The ADSL technology is not sufficient for digital television, TV in HD quality needs the higher transmission capacity of VDSL. This requires providers to rely on a Swisscom wholesale VDSL offering in order to benefit from sufficient bandwidth to offer their customers television over IP.").

${ }^{11}$ Organisation for Economic Co-operation and Developments (OECD), "Financing the Roll-out of Broadband Networks," (June 12, 2014), p. 4, http://www.oecd.org/officialdocuments/publicdisplaydocumentpdf/?cote=DAF/COMP/WP2/WD(2014)17\&docLan guage $=$ En.
} 
the utility companies offered a compelling story: this investment would improve service and ultimately make Swiss cities competitive with cities such as New York and London.

The third disruption in 2007 occurred when cable providers began to adopt the DOCSIS 3.0 standard. This standard for cable modems was adopted in 2006, and enabled speeds of 100 Mbit/s. ${ }^{12}$ This allowed the cable providers to offer "next generation access" services on par with that of the xDSL standards. ${ }^{13}$ According to one of our interviewees, because $90 \%$ of Swiss homes were wired for cable service, it meant that cable providers with $100 \mathrm{Mbit} / \mathrm{s}$ speed were suddenly a threat to Swisscom's broadband dominance.

Because of these new threats to its market share, Swisscom found itself in a bind: invest in new, faster technologies or risk losing customers. As one interviewee described, Swisscom was suddenly facing pressure on multiple sides; both the cable providers and utilities could leverage their existing infrastructure to provide faster broadband than Swisscom was offering. Swisscom responded by announcing a rollout of FTTH in parallel to that of the utilities, ${ }^{14}$ meaning now multiple stakeholders were poised to begin parallel and uncoordinated massive construction projects across the country.

\section{FTTH Roundtables: Improving Coordination Among Stakeholders}

With Swisscom, the utilities, and the cable companies suddenly engaging in competition and costly infrastructure construction projects, the risk for disruption was high. One interviewee described how if the parties operated in an uncoordinated fashion, roads would be torn up, put back, and then torn up again, affecting residents and businesses alike. Similarly, landlords would have to negotiate multiple agreements for access to the premises and installations of fiber hardware. ${ }^{15}$ Moreover, as one interviewee described, Swisscom's demand for exclusive rights to the homes in which they built fiber meant that cable providers risked being shutout. Accordingly, Switzerland faced a complex and unpredictable regulatory and business environment.

\section{A. Why ComCom?}

To address this problem, ComCom announced it was convening an ad hoc series of Roundtable meetings to encourage cooperation among the various stakeholders. In addition to concerns about the disruption, described above, one interviewee said that ComCom believed that left to their own devices, the private sector acting in a regulatory vacuum would roll out FTTH in a slow, uneven, and sub-optimal manner. The concern was that competition and a lack of communication amongst all stakeholders would mean poor organization, delays, and a failure to distribute FTTH evenly across Switzerland. An uneven distribution of FTTH could mean that some regions would be underserved. Additionally ComCom was concerned that absent regulation FTTH would be deployed with incompatible technologies, which would lock

\footnotetext{
${ }^{12}$ Greg White, "DOCSIS 3.0-The road to $100 \mathrm{Mbps,"} \mathrm{CableLabs} \mathrm{Presentation} \mathrm{to} \mathrm{the} \mathrm{NANOG} \mathrm{(2008),}$ https://www.nanog.org/meetings/nanog43/presentations/GWhite_DOCSIS_rev_N43.pdf.

${ }^{13}$ Fibre to the Home Council Europe, Business Committee, "FTTH Business Guide," (February 2012), http://www.ftthcouncilmena.org/documents/Reports/FTTH-Business-Guide-2012-V3.0-English.pdf.

14 "Financing the Roll-Out of Broadband Networks," p. 4.

${ }^{15}$ Swiss Federal Communications Commission, "Interview with Marc Furrer of the ComCom," (September 1, 2009), http://www.comcom.admin.ch/dokumentation/00442/00775/index.html?lang=en.
} 
customers into particular companies and create de facto monopolies. ComCom believed coordination would be necessary to ensure the fiber rollout occurred as quickly and efficiently as possible, while maintaining competition in the marketplace. ${ }^{16}$

At first glance ComCom was ill equipped to deliver such coordination among the stakeholders. Although ComCom had primary responsibility for overseeing the communications market in general, it lacked regulatory authority over FTTH. The Swiss Telecommunications Act did not specify that ComCom had the power to regulate fiber optic networks. This meant that ComCom could not formally compel the stakeholders to join the Roundtables and could not enforce any decisions that emerged from it. Consequently, ComCom's role was, in the literal sense, limited to that of a facilitator, not a regulator. The other political body that could have taken a lead in regulating the FTTH rollout was the Federal Council- the seven-member body that comprises the federal government of Switzerland. However, the Federal Council chose not to intervene. When ComCom requested that the Federal Council grant it additional regulatory authority, the Federal Council deferred, claiming that it would be premature in light of the unbundling legislation passed in $2007 .^{17}$

Despite its limited regulatory power over fiber optic networks, ComCom was competent in the area of telecommunications policy, and was perceived by at least some participants as having legitimate authority over the field. Arguably this placed ComCom in a better position than other regulatory bodies to lead the Roundtables. Other governmental regulators, such as the Federal Office of Communications (OFCOM), still played a significant supportive role. ${ }^{18}$ In its day-today responsibilities, OFCOM "prepares everything that the Federal Council and the Federal Communications Commission need to make policy decisions,"19 and frequently cooperates with ComCom in most regulatory matters. ${ }^{20}$ In the Roundtables, OFCOM organized the technicallyfocused working groups (described below), and provided additional resources and administrative capacity to the leanly staffed ComCom.

Although participation in the Roundtables was voluntary and ComCom lacked specific regulatory powers over fiber optic networks, ComCom was still perceived to have some authority. Other regulators with potential levers of control kept a watchful eye on developments from a background vantage during ComCom's facilitation. While no specific threats appear to have been made, the Roundtables formed under the looming possibility of regulatory action, which undoubtedly influenced the willingness of stakeholders to participate in the Roundtables. Indeed, one of our interviewees noted that one reason why Swisscom participated in the Roundtables was to avoid regulatory scrutiny. The Competition Commission (ComCo) appears to have played an important role in this regard. As Switzerland's antitrust regulator, ComCo is responsible for "monitoring dominant companies for signs of anti-competitive conduct," 21 which it enforces through civil fines. Just one year before the Roundtables were convened, ComCo

\footnotetext{
${ }^{16}$ It is important to note that not all interviewees viewed ComCom's role so charitably. One individual suggested that ComCom was primarily interested in keeping itself relevant in the FTTH telecom space despite a lack of legal authority.

17 "Next Generation Connectivity," p. 320.

${ }^{18}$ OFCOM, "Why is OFCOM needed?," http://www.ofcom.admin.ch/org/00577/index.html?lang=en ("OFCOM's mission is to guarantee that market forces have full play ... [and] attends to these matters on behalf of" ComCom.)

${ }^{19}$ OFCOM, "OFCOM as the regulatory body," http://www.ofcom.admin.ch/org/00598/index.html?lang=en

20 “Swiss ComCom's Annual Report 2007," p. 14.

${ }^{21}$ Swiss Competition Commission (ComCo), http://www.weko.admin.ch/index.html?lang=en\&.
} 
fined Swisscom Mobile CHF 333 million, approximately USD 372.3 million. ${ }^{22}$ ComCo also reviewed the 2010 and 2011 agreements between Swisscom and the utilities that emerged from the Roundtables. ${ }^{23}$

\section{B. The Goals of the Roundtables}

ComCom convened Roundtables in response to a particular problem: the lack of coordination in the rollout of FTTH in Switzerland. Prior to 2008, FTTH simply was not a pressing concern to ComCom (or Swisscom for that matter) ${ }^{24}$ The Roundtables represent an ad hoc distributed governance group formed in response to the identification of an emerging issue. To address this issue, the stated mission of the Roundtables was to define the framework conditions that would allow coordinated development of the optical fiber network. ${ }^{25}$ According to an interviewee who attended the meetings, much of the work of the Roundtables centered around issues of competition in the telecommunications market. However, other key issues covered included the standardization of installations, coordinated network construction, and customers' rights to carrier selection.

In a broader perspective, the Roundtables served as a discussion platform for industry and regulators. It is important to note that the Roundtables were occurring in parallel with FTTH rollout and the signing of cooperation agreements between the stakeholders. ${ }^{26}$ The issues that the Roundtables were addressing were past, present, and future concerns. Accordingly, the Roundtables were not a hurdle that the stakeholders had to clear, but a dialogue that could speed up the process, reduce friction, and ultimately generate better outcomes for both customers and providers.

\section{Beginning the Roundtables}

In 2008, when ComCom convened the first of the Roundtables, it first had to decide who to include in the process. According to one interviewee, ComCom needed to balance between having perspectives and voices at the table so that outcomes would be perceived as legitimate, and having so many voices that consensus would be impossible. The list of participants is not public, but in public statements ComCom and OFCOM asserted that "all the players in the industry (telecommunications companies, electricity providers, cable operators and landlords) joined the effort to define the framework conditions which will allow coordinated development of the optical fibre network." ${ }^{27}$

According to one interviewee, statements about "all the players" participating may be a bit misleading. Instead, ComCom carefully selected the participants, inviting only the largest actors in the market and those who had already begun making FTTH investments. This excluded a large

\footnotetext{
${ }^{22}$ Competition Commission, "Swiss Competition Commission imposes a fine of 333 million Francs on Swisscom Mobile," Federal Administration News, (February 16, 2007), https://www.news.admin.ch/message/index.html?lang=en\&msg-id=10863.

23 "Financing the Roll-Out of Broadband Networks," p. 5.

${ }^{24}$ See "Swiss ComCom's Annual Report 2007," p.6, 15 (mentioning FTTH only twice in relation to other European countries).

${ }^{25}$ ComCom, “"Fibre to the Home' round table — an initial status report," (June 10, 2009), http://www.comcom.admin.ch/aktuell/00429/00457/00560/index.html?lang=en\&msg-id=29395.

${ }^{26}$ See "Financing the Roll-Out of Broadband Networks," p. 4; "Next Generation Connectivity," p. 206-09.

${ }^{27}$ OFCOM, "Deployment of Optical Fibre in Switzerland," http://www.bakom.admin.ch/themen/technologie/01397/03044/index.html?lang=en.
} 
number of potential stakeholders, including all industry associations and advocacy groups, who were upset that they were left out. Every stakeholder that ComCom invited to the Roundtables accepted; one interviewee speculated that the high acceptance rate was in part because an invitation to join a select group of powerbrokers was viewed as prestigious. Ultimately, only about 16 or 17 individuals attended each meeting of the Roundtables.

Given the selection process and the stakes, the barriers for both entry and exit were fairly high. Not only were the participants to the Roundtable handpicked, but also the main meetings required the attendance of the CEO of the invited company or organization. As one interviewee described, companies were not allowed to send delegates or others in place of the CEO; if the CEO was sick, they couldn't participate at that particular meeting. Barriers to exit were similarly high. Once a stakeholder accepted an invitation to join the Roundtables, walking away from the negotiations could be perceived as non-cooperative among the relatively small set of players involved in the FTTH deployment. Moreover, walking away from the table would have meant losing a voice in a process in which large financial interests were at stake.

Although many potential stakeholders were not invited to participate directly, ComCom took steps to try to represent their views within the meetings. For example, according to one interviewee, several groups were invited to presentations at meetings in order to ensure that their perspectives or interests would be raised. Additionally, ComCom conducted extensive bilateral, backchannel conversations with all stakeholders, both those participating and not participating in the Roundtables. According to one interviewee, ComCom used the backchannel conversations so that it could identify the concerns of those not present and ensure that the key issues were acknowledged and addressed during each meeting. ComCom also conducted backchannel discussions with those participating to take stock of priorities and issues, and to determine the feasibility of proposed solutions.

\begin{tabular}{|c|l|l|l|l|}
\hline & \multicolumn{1}{|c|}{ ComCom } & \multicolumn{1}{c|}{ Swisscom } & Cable providers & Utilities \\
\hline \multirow{1}{*}{$\begin{array}{l}\text { Motivating } \\
\text { Interests } \\
\text { competitive } \\
\text { marketplace }\end{array}$} & $\begin{array}{l}\text { Avoid antitrust and } \\
\text { other regulations } \\
\text { Gain sufficient } \\
\text { Ensure } \\
\text { technological } \\
\text { standards allow } \\
\text { consumers to } \\
\text { easily switch } \\
\text { providers } \\
\text { investment in } \\
\text { infrastructure }\end{array}$ & $\begin{array}{l}\text { Ensure } \\
\text { continued access } \\
\text { to the } \\
\text { infrastructure } \\
\text { within homes } \\
\text { and buildings }\end{array}$ & $\begin{array}{l}\text { Maximize return } \\
\text { on investment in } \\
\text { infrastructure }\end{array}$ \\
& $\begin{array}{l}\text { Project relevance } \\
\text { and influence } \\
\text { into a new field }\end{array}$ & & & \\
& & & \\
\end{tabular}

Figure 1: Motivations for Round Table Stakeholders 


\section{Roundtables in Action}

\section{A. The Structure of the Roundtables}

Because there were no existing structures to handle coordinating FTTH deployment, and no government agencies with direct regulatory authority over the matter, the Roundtables presented both an opportunity and challenge. The opportunity was in designing a set of ad hoc structures that could directly address specific needs of the FTTH stakeholders. The challenge was in designing the structures so that they would be considered legitimate. ComCom decided to adopt a tiered approach that would separate the high-level policy and strategic questions from the more technical questions. Under this structure, the main Roundtables were limited to CEO-level representatives. The CEO-level group was chaired by the ComCom President Marc Furrer and addressed the policy and strategic questions. Simultaneously, OFCOM organized a set of complimentary working groups that focused on technical matters of a few varieties. For instance, technical working groups addressed interconnection standards, while legal working groups addresses contracting and regulatory technicalities. According to one interviewee, this two-tiered structure allowed technical issues to be resolved quickly, while higher-level participants could negotiate the more political strategy issues.

\section{B. The Roundtable Meetings}

The Roundtables met nine times between 2008 and 2012. Despite the significant monetary value of the FTTH deployment, the Roundtables themselves were a relatively minor investment of resources. The meetings were held in Solothurn, a small town north of ComCom's headquarters in Bern, in an old mansion. According to an interviewee, this location was picked because it was far enough away from the participants' offices that they would be likely to the stay for the entirety of each meeting instead of departing early to attend other meetings.

ComCom proposed the agenda for each Roundtable meeting. Approximately five days in advance of each meeting, ComCom would distribute an agenda and solicit feedback from the participants. According to one interviewee, the agenda was fairly straightforward in the sense that there was consensus on the issues to be discussed and the order in which they needed to be address. Any additions or alterations to the agenda were generally adopted by consensus as needed.

According to one of our interviewees, the central topic of the Roundtables was resolving the terms of use upon which data would flow through fiber optic cables into the home. The largest point of contention related to open access versus exclusivity. The utility companies largely wanted to adopt an open access model, where they would rent the fiber infrastructure to any service provider, leaving the consumer with equal choice among competitors. ${ }^{28}$ In contrast, Swisscom did not want to open up to competitors the fiber it was deploying, as it was forced to do in years prior with copper wire in the local loop unbundling legislation. Thus, Swisscom sought some degree of exclusivity for their services, to ensure that they could justify the substantive investments in infrastructure. Finally, the cable operators generally sought to maintain access to residences and not be shut out by exclusivity agreements between the owners

\footnotetext{
${ }^{28}$ See "Next Generation Connectivity," p. 206 (describing the Openaxs coalition "committed to the concept of open access and with the goal to promote fiber-optic networks in general and FTTH in particular based on the principles of fair competition and consumer choice.").
} 
of the fiber and the landlords or homeowners. The Roundtables served as a means for balancing these competing interests.

\section{Confidentiality}

There were only two rules governing the operation of the Roundtables. One of those rules, already addressed, was that only one person was permitted around the table from each participating organization, and that person must hold a CEO-level position. Any accompanying staff were instructed to wait outside of the room during the meetings. The second rule, according to interviewees, was that none of the participants would talk to the media concerning the content of the discussions; instead, ComCom would act as sole representative of the Roundtables to the public.

In theory both of these rules were in place to enable a free and open discussion, and create an environment where consensus and compromise was possible, without participants needing to seek approval from superiors. Attempting to reach consensus on the coordination FTTH deployment would require the participants to disclose information about their corporate strategy, and accordingly it was believed that a closed-door discussion would best facilitate reaching an outcome. Although using closed-door meetings may have facilitated a more open discussion, according to at least one participant, it came at the cost of perceived legitimacy for those stakeholders who believed their views were not adequately addressed.

\section{ComCom as Mediator?}

ComCom's effectiveness as the convener of the Roundtables is a matter of some debate. According to one participant, ComCom viewed the Roundtables as a multi-party negotiation, and assumed the role of neutral mediator trying to facilitate a consensus outcome. To this end, ComCom invested substantial time before and after each meeting in bilateral dialogue with stakeholders, both those participating and not. Although this work was not publicized, according to one perspective, these conversations enabled ComCom to mediate between the various parties. In particular, during these bilateral discussions ComCom could engage in negotiations with stakeholders, identify potential stumbling blocks to consensus agreements, and float proposals with stakeholders to assess their reactions.

Another interviewee, however, suggested that the Roundtables were largely a public relations event that enabled ComCom to appear as though it were influencing deployment of FTTH. For instance, this participant said that the full list of agenda items was rarely covered due to time constraints. Despite the fact that the Roundtables failed to cover many of the agenda items, after each meeting, ComCom would publish a press release highlighting areas of agreement. The participant believed on at least some occasions the press releases claimed successes prematurely. The lack of public documentation from the meetings themselves, combined with the fact that the participants agreed that ComCom would be the sole outlet for information regarding the discussions, make it challenging to independently confirm the accuracy of these statements and the effectiveness of ComCom's role more generally.

\section{E. Working Groups}

As described above, OFCOM supported the Roundtables by managing a series of working groups that focused on the more technical aspects of the Roundtables. The working groups were divided into topical areas, with two working groups dedicated to the infrastructure layer (Layer 
1), two working groups dedicated to the services layer (Layer 2), and one for legal issues. As described by OFCOM ${ }^{29}$ the mission of each working group is as follows:

- Working Group L1: Define standards for optical fiber cabling inside buildings

- Working Group L1B: Determine the transfer points in FTTH networks

- Working Group L2: Discuss common points relevant for the industry concerning access to services and L2, particularly enabling access for alternative providers who do not have their own network

- Working Group L2B: Establish a universal platform for the ordering of, or migration to, optical fiber and transport services

- Working Group 3: Draw up recommendations for arranging contracts between network operators and building owners

Generally, each working group created a set of recommendations or proposed standards that address its assigned topic. In most cases, the documents included an executive summary, introduction, definitions, scope, topic specifics, recommendations, next steps, graphs or other visual aids, and references. Each working group had the flexibility to shape their guidelines to fit their specific mission.

Beyond simply providing a targeted forum for resolving less strategic issues, the working groups offered two additional benefits. First, they provided a venue for participation for some of those who were not invited to the CEO-level Roundtables. Second, the technical guidelines and model contracts produced by the working groups are best practice documents that can serve as resources for others, both in Switzerland and beyond, about possible approaches to coordinating deployment of FTTH.

\section{Decision Making and Outcomes}

\section{A. Use of Consensus}

Ultimately, the Roundtables led to the consensus adoption of a "four-fiber" solution, which was originally proposed by Swisscom. Consensus was a key aspect of the Roundtables both by design and out of necessity. According to one interviewee, ComCom's lack of regulatory authority was, ultimately, a benefit. Because there was no actor that could enforce the decisions of the Roundtables, any outcomes would need the support of all of the participants in order to be successful. Today, Switzerland has deployed FTTH far faster than many other European countries, in part because while other countries have relied upon regulation to force FTTH buildout, Switzerland's rollout was done with the support of all key stakeholders.

Although consensus was important, not all of the Roundtable participants shared equally in every decision. For example an interviewee noted that when the Roundtables addressed the issue of cost sharing, Swisscom and the utilities were the primary parties to the decision, with the cable companies playing a more minor role because they were building new infrastructure. Our interviewees did note that every participant participated in good faith, even when disappointed with a particular outcome. Thus, the Roundtables made decisions using consensus, but not every issue required the full support or involvement of every party.

\footnotetext{
${ }^{29}$ Federal Office of Communications, "FTTH Working Groups," http://www.bakom.admin.ch/themen/technologie/01397/03044/03046/index.html?lang=en.
} 


\section{B. Defining Success}

ComCom's public statements assert that the Roundtables were successful in achieving its stated mission and goals, though our interviews indicated some skepticism. In its press releases, ComCom claimed the following successes: ${ }^{30}$

- The network architecture was created in a coordinated manner, encouraging competition without duplication.

- Uniform technical standards were established, including the four-fiber model proposed by Swisscom.

- A model contract was made available for property owners and networks operators governing legal and financial aspects of FTTH installation.

The four-fiber model that the parties adopted was particularly significant as a mechanism for reconciling the stakeholders' divergent objectives of open access (the utility companies) and exclusivity (Swisscom). Under this model, multiple lines of fiber would be deployed to each home, with the costs and uses shared between the participants. For instance, if Swisscom built FTTH in a city, with investment from the local utility, Swisscom would own all four fibers and get exclusive access to one or two fibers, the utility would have an indefeasible right to use a fiber, and the remaining fiber could be rented wholesale to other competitors. Thus, Swisscom had exclusive right to a fiber, and the utilities were able to offer open access services on their fibers.

By January 2012, ComCom and the other Roundtable participants agreed that the meetings were no longer necessary. According to one interviewee, the Roundtables were never formally concluded, and the option remains to reconvene if the parties believed that the agreements were not working as intended, or if additional coordination became necessary.

By many metrics, the rollout of FTTH in Switzerland appears to have been quite successful. According to ComCom's 2013 Annual Report, ${ }^{31}$ 750,000 homes and businesses now have fiber access in Switzerland, and that number is expected to grow by 200,000 per year. Additionally, all providers have access to the network on equal terms via the open access fibers and at multiple levels of the network, and users with fiber have the ability to choose their providers.

Despite impressive numbers of FTTH deployment, it is not clear to what extent the success is attributable to the Roundtables. For example, although the four-fiber model was agreed to as part of the Roundtables, it was not initially proposed in the Roundtables. In fact, Swisscom announced this model in 2008, when the Roundtables were just beginning. ${ }^{32}$ The first Roundtable press release to mention a multiple fiber model was in October 2009. ${ }^{33}$ Consequently, it is difficult to know whether widespread adoption of the four-fiber model was an

\footnotetext{
${ }^{30}$ ComCom, "Fiber to the Home_-Press Releases Regarding FTTH," http://www.comcom.admin.ch/themen/00769/index.html?lang=en.

${ }^{31}$ ComCom, “Activity Report ComCom 2013,” (2013), http://www.comcom.admin.ch/dokumentation/00564/index.html?lang=en.

${ }^{32}$ Swisscom, "Into the fibre-optic future with 'fibre Suisse'," Press Release, (December 9, 2008), http://www.swisscom.ch/de/about/medien/pressreleases/2008/12/20081209_01_Mit_fibre_suisse_in_die_Glasfaserzukunft.html.

${ }^{33}$ ComCom, "Fibre to the Home round table_an initial status report," (October 6, 2009), http://www.comcom.admin.ch/aktuell/00429/00457/00560/index.html?lang=en\&msg-id=29395.
} 
outcome of the Roundtables, or simply a solution that would have occurred in the absence of any intervention.

More generally, it is not clear that the four-fiber model has worked as a mechanism for generating competition in the marketplace. According to one participant, the open access fibers that the utilities control are largely dark (unused). This individual speculated that one reason why the utilities have been unable to rent their fiber is because they failed to sufficiently grasp the telecommunications market. Since the utilities are all local, any service providers who wanted to use the open access fiber would have to negotiate separate agreements with each locality in which they want to offer service. In contrast, in most regions Swisscom controls two fibers, and can offer the excess capacity on a national scale-an offer that is more appealing than negotiating hundreds of separate agreements.

Another possibility for why the utilities companies have been unable to profit from their fiber was the uneven leverage of the participants during the Roundtables. According to one participant, Swisscom and the cable providers were more successful at representing their interests and obtaining their desired results than the utilities, who often lacked necessary technical knowledge. In some cases the utility representatives to the Roundtables did not understand technical operation of a fiber network, making it difficult for them to advocate on behalf of their interests.

Not all of the participants had such a pessimistic interpretation of the events. One interviewee, suggested that the FTTH rollout is still in its "early days." In the long run, the participant believed that the fiber would be used because the need for high-speed bandwidth would only continue to increase. Moreover, the fact that the deployment and cooperation was accomplished without government regulation was a success in itself, and according to the participant, may be why FTTH deployment has occurred far faster than in other European nations.

All of our interviewees agreed that the Roundtables were useful for fact-finding, convening stakeholders, and defining regulatory standards. From here, however, perspectives diverge on other topics. One view was that the Roundtables could have served as a valuable input for the development of a law or regulation amenable to all players; in the absence of such a law it left an unregulated environment that overwhelmingly benefits the market dominant Swisscom. Another view, however, was that all parties benefited from the outcome of the Roundtables and that regulation and government intervention would have only served to reduce the incentives for FTTH development in Switzerland.

\section{Observations on Particular Features}

It is possible to assess some aspects of the Roundtables as a means of deploying FTTH in Switzerland. As the discussion above indicates, it is perhaps premature to determine whether the deployment of FTTH in Switzerland was itself a success, but based upon interviews with participants and a review of the process of convening the Roundtables, it is possible to make some observations with regards to the elements that worked better and worse than others, as well as offer a considerations that could be instructive. 


\section{A. Regulation May Not Be Necessary to Compel Participation Where Market Incentives are Sufficiently Strong}

The stakeholders that participated in the Roundtables did not do so because they were forced by law to attend. Instead, they appeared to recognize the opportunity that the Roundtables offered, particularly as a means for reaching agreement on cost-sharing, and reducing duplication of effort. Additionally, our interviews suggested that Swisscom saw the Roundtables as a means to avoid antitrust regulation, suggesting that in this case the potential for regulation was just as effective as regulation itself.

\section{B. Participants May View the Value of Transparency Differently}

Some participants felt that the Roundtables were opaque. While the decisions were published via press releases and working group outputs, the participant selection process, the identities of the participants, and the decision-making processes were not public. Others, however, viewed the lack of transparency as a necessity in order to reach best outcomes from a policy standpoint.

\section{Reducing Rules or Procedures Comes With a Tradeoff}

The convening and operation of the Roundtables were fairly informal. In part this seems to be a function of the fact that there was there was no precedent to follow from the creation of similar organizations in Switzerland. According to our interviews, the informality also seemed to follow from a mix of ComCom trying to ensure that the stakeholders could define through consensus the role of the Roundtables, and ComCom lacking the authority to impose a definition in the first place. Regardless of the reason, the results of this informality were mixed according to participants. Some felt that the informal approach provided efficiency and allowed the stakeholders to have ownership over the process, but others felt that the informality enabled the more experienced stakeholders to take advantage of the utilities, because the rules offered no protections or constraints on power.

\section{Separation of Strategic and Technical Domains May Be an Effective Approach to Addressing Complex Issues}

The Roundtable was distributed due to the fact that it separated the high-level strategic issues, addressed by the CEOs, from the more technical matters, addressed by the working groups. These two distributed elements were cooperative but were run by two separate entities, providing some independence and avoiding a bottleneck of decision making. OFCOM, which managed the working groups, declined to be interviewed for this case study; further research would explore in more detail how OFCOM managed and coordinated the working groups, particularly with respect to the decisions emerging from the CEO-level sessions.

\section{E. Skilled Facilitation May Address Issues of Incomplete Representation}

Although ComCom invited the stakeholders that it perceived as necessary, it did not include all individuals and groups that had a stake in FTTH deployment. For example, industry associations, community or neighborhood groups concerned about construction noise or environmental factors, and landlords and homeowners associations were not invited. According to an interviewee, the smaller group enabled faster decision making and increased the chances of reaching consensus. An interviewee suggested that many who were left out of the process were 
initially unhappy; the Chair of the Roundtables, ComCom's Marc Furrer, apparently took steps in order to engage bilaterally with all of these excluded stakeholders, and then brought their perspectives back to the Roundtables.

\section{F. Ad Hoc Groups May Be Built on Already Established Structures}

Prior to the Roundtables, ComCom and OFCOM had an existing formal relationship. Although the Roundtables and affiliated working groups were new, the idea of the two organizations working in tandem on telecommunications issues was not.

\section{G. Subject Knowledge May Be an Important Strategic Resource}

According to one participant, despite significant monetary resources, the utility companies struggled to effectively advance their interests in a forum dominated by stakeholders with extensive experience in the telecommunications industry. Accordingly, financial resources may not be sufficient to ensure meaningful participation, if such resources are not used to mitigate a knowledge deficit.

\section{Conclusion}

The Roundtables are an interesting example of an ad hoc, distributed governance organization. In particular, ComCom faced the challenge of achieving consensus among its participants and doing so in the absence of formal regulation enforcement authority. Without conducting more empirical research, it is perhaps not possible to conclude that the Roundtables caused the FTTH deployment in Switzerland to be successful. That said, the Roundtables do provide some helpful insights into governance models. For example, the Roundtables are particularly informative on the topics of mapping issues to the correct stakeholders and solutions, the role enabling organizations such as technical experts can play in developing policies, and coordination between actors at both national and local levels.

The Roundtables highlight the challenge of matching governance problems with the right organizations, experts, networks, and governing bodies/entities best able to develop legitimate, effective and efficient solutions. In this example, despite the absence of any formal authority to regulate FTTH, ComCom took the lead in creating a forum to address the issue. The financial self-interest of the parties, the prestige of the dialogue, and the network of regulatory bodies operating in the background, created an environment in which all stakeholders that ComCom invited joined in willingly and contributed meaningfully. That said, ComCom did not invite all stakeholders to participate in the Roundtables; it exercised its discretion in mapping the issue onto the appropriate stakeholders. Although this decision was not initially received well, it appears that most parties were mollified through bilateral conversations with ComCom, or through seeing their views represented in either other parties or the final outputs.

The Roundtables also highlight the important role that expert enablers can play in the formation of policy. Enablers are often organizations or resources that allow governance groups to quickly access information and expertise that can guide the workings of the group. For example, enablers may take the form of forums and dialogues (which facilitate broad engagement), expert communities (which facilitate targeted engagement), and capacity development and toolkits (which facilitate empowered engagement). In the Roundtables, the work of CEO-level 
discussions were complemented through OFCOM's working groups, which were designed to facilitate the targeted engagement of experts in technical and legal matters related to FTTH deployment.

Finally, the Roundtables highlight the importance of coordination between local and national entities in order to coordinate decision making and lower friction. The Roundtables involved diverse group of players spanning the local, cantonal, and national levels, which represented a variety of horizontal and vertical market players, in coordinating a massive national infrastructure project. As noted at the beginning of this case study, one of the sparks that made the Roundtables necessary was a quintessentially local decision: local utility companies deciding to invest in local infrastructure projects. However, all players - both local and nationalrecognized the opportunity that coordination could play in improving outcomes and lowering costs.

From a research perspective, a key unanswered question remains the counterfactual question of what would have occurred with Switzerland's FTTH deployment had the Roundtables not provided an opportunity for coordination? Would the parties have entered into bilateral coordination in the absence of the Roundtables? Would the deployment of FTTH have been more expensive, slower, or disruptive? Would all of the stakeholders' views been similarly represented? Only by trying to piece together that alternative scenario would it be possible to fairly evaluate the individual decisions made in the context of the Roundtables. Although it is impossible to reconstruct such a counterfactual, further research would benefit from additional interviews, especially with those who did not attend the Roundtables. 


\section{Appendix L: \\ Turkish Internet Improvement Board}




\title{
NoC Internet Governance Case Studies Series: Turkish Internet Improvement Board
}

\author{
Leyla Keser and Mehmet Bedii Kaya \\ IT Law Institute, Istanbul Bilgi University
}

\section{Editorial Note: Context, Character, and Purpose of the Case Study}

This case study is part of a globally coordinated, independent academic research pilot project by the Global Network of Interdisciplinary Internet \& Society Research Centers (NoC). Facilitated by the Berkman Center for Internet \& Society at Harvard University, this study examines existing multistakeholder governance groups with the goal of informing the future evolution of the Internet governance ecosystem. Building upon the NETmundial Principles and Roadmap, it contributes to current policy debates at the international level, including the Internet Governance Forum, the NETmundial

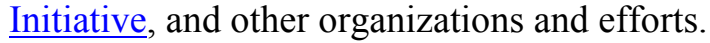

Internet governance is an increasingly complex concept that operates at multiple levels and in different dimensions, making it necessary to have a better understanding of both how multistakeholder governance groups operate and how they best achieve their goals. With this need in mind, at a point where the future of Internet governance is being re-envisioned, colleagues from several $\mathrm{NoC}$ institutions around the world have written twelve case studies examining a geographically and topically diverse set of local, national, and international governance models, components, and mechanisms from within and outside of the sphere of Internet governance. Key findings from these cases are summarized in a synthesis paper, which aims to deepen our understanding of the formation, operation, and critical success factors of governance groups and even challenge conventional thinking.

The research, based on twelve case studies, suggests that there is no single best-fit model for multistakeholder governance groups that can be applied in all situations. Rather, it reveals a range of approaches, mechanisms, and tools available for both the formation and operation of such groups. The analysis demonstrates that the success of governance groups depends to a large degree on the careful selection, deployment, and management of suitable instruments from this "toolbox." As governance groups pass through different phases of operation, conveners and facilitators must remain alert to changes in circumstances that necessitate adjustments to the approaches, mechanisms, and tools that they deploy in order to address evolving challenges from inside and outside. This case study provides insights into how those instruments can be deployed and adjusted over time within such groups, and highlights how their interactions with important contextual factors may be successfully managed within given resource restraints.

The research effort is grounded in a diversity of global perspectives and collaborative research techniques. Adhering to objective and independent academic standards, it aspires to be useful, actionable, and timely for policymakers and stakeholders. More broadly, the Network of Centers seeks to contribute to a more generalized vision and longer-term strategy for academia regarding its roles in research, facilitation and convening, and education in and communication about the Internet age.

For additional information on the initiative, please contact Urs Gasser, Berkman Center for Internet \& Society, at ugasser@cyber.law.harvard.edu. 


\begin{abstract}
This case study focuses on a multistakeholder working group that the Turkish Internet Improvement Board created in order to generate innovative, bottom-up approaches for fixing the Internet law in Turkey. In 2007, Turkey adopted its first comprehensive Internet content regulation legislation: Law 5651, the "Regulation of Publications on the Internet and Combating Crimes Committed by Means of Such Publications." Since its adoption, Law 5651 has come under scrutiny for enabling overly broad restrictions on web content, its establishment of criminal liability for hosting providers who fail to adequately restrict content, the threats it posed to Internet-related innovation in the country, the cybersecurity vulnerabilities that resulted from the widespread use of circumvention tools by Turkish Internet users in response to content restrictions under the law, and related data protection concerns. The law also called for the creation of the Internet Improvement Board. In 2011, the Board created the " 5651 Working Group," comprised of seven representatives from government, civil society, academia, and the private sector, to explore possible solutions to these problems. This case study examines how the working group collaborated with a broader group of stakeholders to develop a resolution, delivered to the Ministry of Transportation, Maritime Affairs, and Communication, proposing amendments to Law 5651.
\end{abstract}




\section{Table of Contents}

I. Regulation of the Internet in Turkey .................................................................................. 1

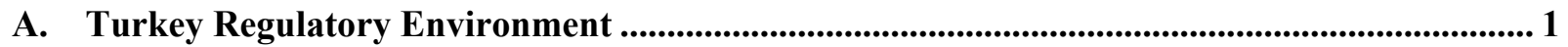

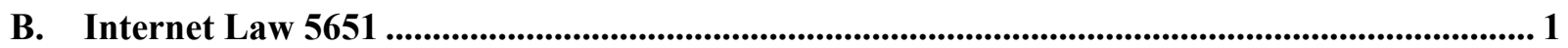

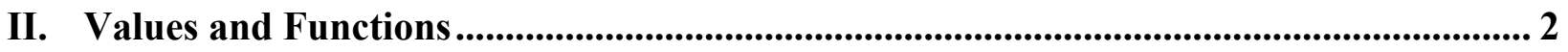

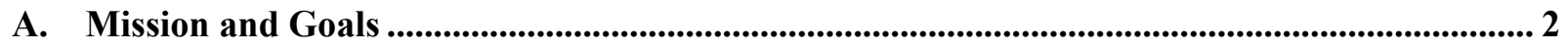

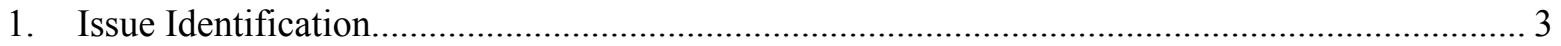

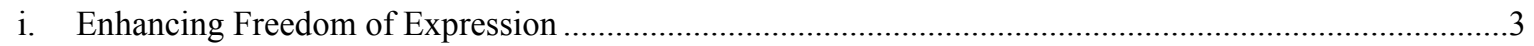

ii. Protecting Hosting Providers From Excessive Criminal Liability .......................................................

iii. Ensuring a Suitable Environment For International Investment …...................................................

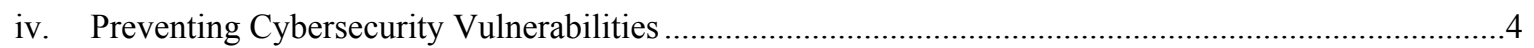

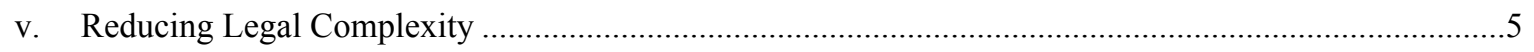

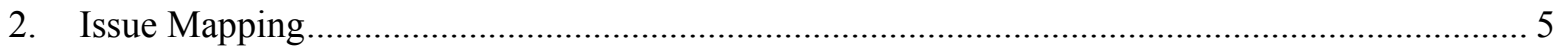

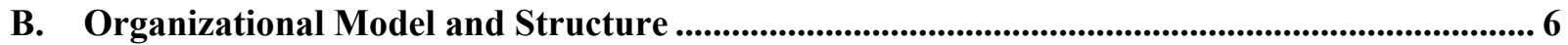

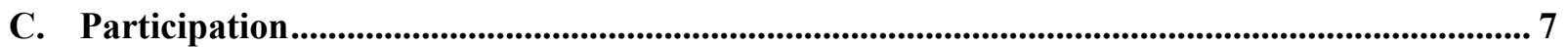

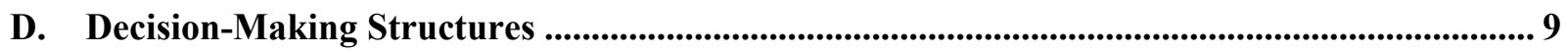

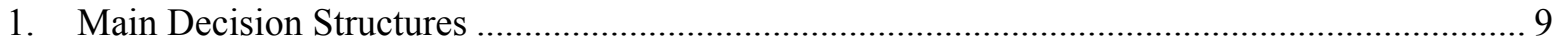

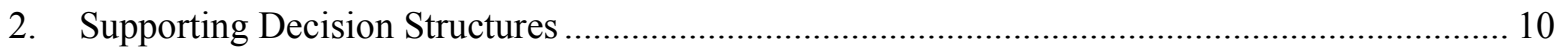

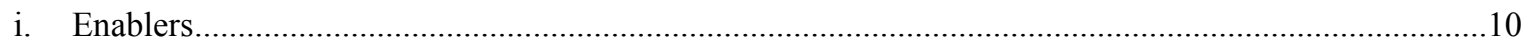

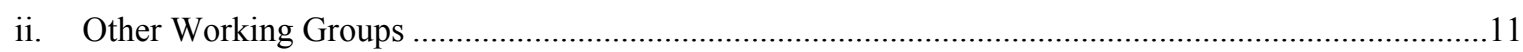

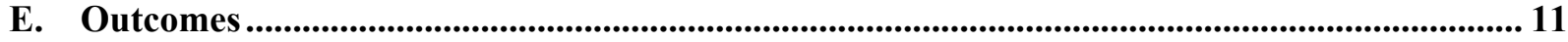

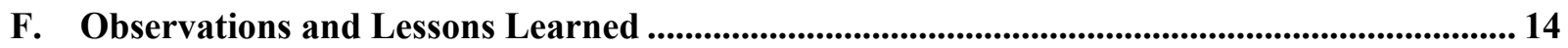




\section{Regulation of the Internet in Turkey}

\section{A. Turkey Regulatory Environment}

Politics in Turkey take place within the framework of a parliamentary representative democratic republic and a multi-party system. The President of Turkey is the head of state that holds a largely ceremonial role but has substantial liberum veto.

Turkey's political system is based on separation of powers; the Cabinet exercises executive power, legislative power is vested in the Grand National Assembly of Turkey, and the judiciary is independent of the executive and the legislature.

The Turkish government executes its power through different Ministries on different matters. According to the principle of separation of roles and responsibilities, the Ministry of Transportation, Maritime Affairs and Communication" (hereafter "the ministry") is the main body responsible for the Internet, telecommunication, and communication. This Ministry develops Internet governance policy, rules, and approaches. There is a sub-institution under the Ministry, which is responsible for making these policies concrete. This sub-institution is the Turkish Information Technologies and Communication Authority (hereafter "ITCA"). ${ }^{2}$. The Turkish ITCA has a specific department for Internet and governance issues, which we call the Turkish Telecommunication and Communication Presidency (hereafter “TIB”).

\section{B. Internet Law 5651}

Law 5651, entitled the "Regulation of Publications on the Internet and Combating Crimes Committed by Means of Such Publications" (hereafter "Law 5651"), was passed on 4 May $2007^{3}$. TIB is the body responsible for the implementation of the Law. TIB has a presidency established under Turkish ITCA, which is the main regulatory body of telecommunication in Turkey and is in charge of policy making, regulation, and operation. TIB was established within the Turkish ITCA in August 2005 and became fully functional in July 2006. The main purpose of its formation was to centralize into a single unit the surveillance of communications and the execution of "interception of communications" warrants subject to Laws 2559, 2803, 2937, and 5271. Under the Law 5651, the TIB was chosen as the organization responsible for monitoring Internet content and executing blocking orders issued by courts, judges, and public prosecutors. The TIB has authority to issue administrative blocking orders with regards to certain Internet content hosted in Turkey and with regard to websites hosted abroad in relation to crimes listed in Article 8 of Law 5651, e.g. sexual harassment of children, obscenity, and prostitution. Furthermore, the TIB is entitled to determine the nature, timing, and procedures concerning content monitoring systems on the Internet, and it determines the minimum criteria with regard to the production of hardware or software for filtering, screening, and monitoring purposes.

Under the Ministry there are several permanent boards, which provide the Ministry with close relationships with different stakeholders across a number of sectors and which were established based on a specific provision of the Decree Law Concerning the Organization and Duties of the

\footnotetext{
${ }^{1}$ www.ubak.gov.tr.

${ }^{2}$ www.btk.gov.tr.

${ }^{3}$ http://www.tib.gov.tr/tr/tr-menu-42-kanunlar.html.
} 
Ministry. ${ }^{4}$ One of these boards is the Internet Improvement Board (hereafter "the Board") ${ }^{5}$, on which we focus below. This Board was established by the Ministry to create a small multistakeholder group to which the Ministry could hand over policy and regulation related matters on the Internet. The Ministry's approach to Internet governance is to address matters favored by the Board, instead of only governmental organizations. For this reason, Law 5651 has a specific provision that requires continuous collaboration between the Board and the TIB in order to promote increased and safe use of the Internet. This provision of Law 5651 illustrates how the Board is seen a counterweight against the power of the government on Internet related issues.

\section{Values and Functions}

\section{A. Mission and Goals}

Turkey adopted its very first comprehensive Internet content regulation in 2007 by enacting Law 5651. This piece of legislation not only laid down the essentials of Internet content regulation, but also laid out a detailed set of rules in regard to the liability of Internet actors, such as content, hosting, and access providers, and established a specific mechanism, so-called "access restriction", to combat certain crimes. This legal framework was primarily shaped the desire of politicians to find and equilibrium between the prevention of crime, the protection of personal rights, and privacy while maintaining the free flow of information over the Internet. However, over time, the implementation of the Law 5651 has led to undesired outcomes, such as restricting thousands of websites at the DNS level (including YouTube.com), and as a result this law has come to the forefront of the Turkish legal and political agenda.

While there have been public debates about the reform of Law 5651, there has been no consensus about how to conduct the reform process. Most importantly, the issues that will be subject to reform have not been identified properly. Different stakeholders such as national and international private sector companies, ISPs, NGOs, and academia have expressed their views publicly and shared their concerns with public bodies without following a predefined template or guideline $^{6}$. They have underlined the importance of the same objections regarding Law 5651, explained below. Furthermore, different stakeholder groups have arranged spontaneous meetings to discuss the reform of Law 5651. However, due to the lack of predefined templates and the failure to identify key issues, such meetings did not provide concrete outcomes.

The Board, on the other hand, approached this issue systematically and institutionalized Law 5651 reform discussions. The very first step was issue identification and framing the issues to be discussed before any stakeholder group could address them. In this way, the Board established the necessary conditions for stakeholders to address the identified issues. Based on that framework, the Board decided to gather various stakeholders together in a group format (hereafter "the Working Group") in January 2011. The objective of the Working Group was to determine issues with Law 5651, to discuss and find possible solutions, and to formulate these as a draft Law collaboratively with the Board. The name of the Working Group was " 5651 Working

\footnotetext{
${ }^{4} \mathrm{http}: / /$ www.resmigazete.gov.tr/eskiler/2011/11/20111101M1-1.htm.

${ }^{5}$ See www.internetkurulu.org, Article 29/paragraph 6 of the Decree Law Concerning the Organization and Duties of the Ministry.

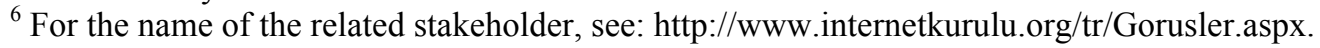


Group", which consisted of all relevant stakeholders - namely government, civil society, academia, private sector, and citizens. As is explained in detail below, the seven members on the Board represented these different stakeholders. Therefore, the membership of the Board overlapped with the membership of the Working Group. However, in order to reach more people from each stakeholder group and include them in the ongoing work on Law 5651, the Board created the Working Group.

The Board and the Working Group performed two roles important for distributed governance: issue identification and mapping the issue onto the appropriate sphere. Collectively, we can call this solution mapping.

The main issue that the Working Group tried to address was the reform of the legal framework of Internet content regulation, i.e. Law 5651. This piece of legislation in particular had significant implications for the following issues, which were identified as important during several meetings of the Board and the Working Group.

\section{Issue Identification}

\section{i. $\quad$ Enhancing Freedom of Expression ${ }^{7}$}

Law 5651 permitted the restriction of access to websites that host illegal content, however, it did not stipulate a specific method of restriction. Due to a lack of the technical infrastructure at the ISP level that would enable restriction based on URLs, websites were restricted based on the DNS and their IP addresses. Such a restriction, however, gave rise to the disproportionate suppression of freedom of expression since DNS and IP address blocks access to entire websites. For instance, the restriction of access to YouTube on 6 March 2007, 17 January 2008, and 29 October 2010 are prominent examples of this issue. Besides Law 5651, the implementation of other legislation that dealt with content regulation, such as Law No. 5846 on Intellectual and Artistic Works, also led to similarly adverse outcomes for freedom of expression. For example, on 24 October 2008 and 14 January 2011 access was restricted to Blogger on the grounds that certain blogs hosted content that infringed on intellectual property, and the government lacked the technical infrastructure that would enable content-specific restriction. Another example of such disproportionate restriction was the restricted access to Google Sites on 23 June 2009. It is noteworthy that The European Court of Human Rights - in the decision of Ahmet Yildirim dated 18 December 2012 - ruled that this restriction against Google Sites was an explicit violation of freedom of expression.

As highlighted in the NetMundial outcome document, freedom of expression, freedom of information and access to information are essential pillars of Internet governance. The approach enshrined in Law 5651, however, significantly restricted these rights since the web sites were blocked on a DNS basis.

\footnotetext{
${ }^{7}$ Relevant stakeholders: All individuals, IT and social media companies, Government, NGOs.
} 


\section{ii. $\quad$ Protecting Hosting Providers From Excessive Criminal Liability ${ }^{8}$}

Another provision of Law 5651 that created controversy was the provision of criminal liability laid out for hosting providers. Under the law, hosting providers who did not restrict access to web sites when ordered could be subject to imprisonment for six months to two years.

Criminal liability that could lead to imprisonment was considered the biggest obstacle to improvement of the hosting landscape in Turkey. In the same vein, since the concept of "hosting providers" was defined so broadly and there were no provisions that would enable different treatment based on the nature of service provided, any website that was operating on the basis of Web 2.0 technologies could be treated as hosting provider and thus be subject to criminal liability. As highlighted under the NetMundial outcome document, intermediary liability limitations should be implemented in a way that respects and promotes economic growth, innovation, creativity, and the free flow of information. The provisions laid down under the Law 5651, though, were far from compliant with the minimum safeguards prescribed by NetMundial.

Furthermore, according to Law 5651, in order to operate as a hosting provider, entities were required to receive administrative permission. No operation was allowed before such permission was granted. Providers who did not have permission but keep providing hosting services could also be subject to severe administrative fines. In conjunction with the broad definition of hosting providers, this requirement added another layer of complication to the controversy. As a result of this provision, many hosting providers transferred their business to other countries, where such restrictions did not exist.

\section{iii. $\quad$ Ensuring a Suitable Environment For International Investment}

Another important controversy with regard to Law 5651 was the interpretation of what was meant by restricting access. Foreign economic operators, in particular social media sites, claimed that Law 5651 did not mandate the deletion of content and geographically based restrictions, such as restricting access to Turkish users. However, this approach was not generally accepted by the Turkish courts, which interpreted restricting access as involving the removal of content from servers by access and/or hosting providers. Due to that provision, many foreign ICT companies hesitated to enter and invest in the Turkish market. In this respect we could say that Law 5651 did not create an Internet environment for sustainable innovation and creativity.

\section{iv. $\quad$ Preventing Cybersecurity Vulnerabilities}

As explained previously, while Law 5651 was in affect the only possible way to block access to illegal content was blocking through DNS. However, this method of restriction was not efficient and could easily be circumvented through different tools, e.g. tunnels, VPNs, and similar tools. Such tools, though, involved significant legal risks since they were redirecting all traffic on the sites to servers abroad. Besides the risk of jeopardizing the privacy of Internet users, such tools also created major cybersecurity vulnerabilities as these kinds of free services often expose Internet users to computer viruses. The wide use of such tools not only created end-user specific problems, but created a country-wide security problem. As a result, Turkey was among the most virus-infected countries in the world.

\footnotetext{
${ }^{8}$ Relevant stakeholders: Hosting providers, platform providers, intermediary service providers.
} 
Taking into consideration such important cyber security leaks, which were clarified by the technical groups, the Working Group offered to add a new provision to Law 5651 that would enable URL banning instead of DNS or IP blocking. The Working Group discussed and analyzed advantages and disadvantages of URL banning. The most significant concern regarding this was the use of Deep Packet Inspection (DPI). In order to enable URL banning, DPI would have to be used by ISPs. While undertaking DPI, all Internet traffic must be monitored, which obviously violates the data protection rights of individuals. Furthermore, the URL restriction method requires a substantial hardware and software investment and a major infrastructure transformation. In that regard, technical challenges emerged as a key barrier for implementing the URL method, since access providers needed to undergo substantial upgrading before the implementation of such content based restriction. Indeed, one of the main reasons for the use of the DNS restriction method rather than URL restriction method was that the DNS method could be implemented without any further hardware and software costs to access providers.

As also highlighted under the NetMundial outcome document, the security, stability, and resilience of the Internet need to be key objectives of all stakeholders in Internet governance. In that regard, NetMundial states that as a universal global resource, the Internet needs to be a secure, stable, resilient, reliable, and trustworthy network, which requires strong cooperation among different stakeholders. Thus, it is noteworthy that all stakeholders considered the prevention of cyber security vulnerabilities in the implementation of Law 5651 a top priority.

v. $\quad$ Reducing Legal Complexity

Aside from Law 5651, over time different laws permitted different institutions and courts to restrict access to Internet content on different grounds. This proliferation of reasons and differentiation of rules added a new layer of complication for stakeholders. Law 5651, when it was first adopted in 2007, succeeded in unifying and simplifying the legal framework regarding regulation of the Internet in Turkey. However, over time, unjustified exemptions were introduced to Law 5651 through different pieces of legislation, which undermined Law 5651 as the main piece of legislation governing the Internet in Turkey.

This diversity of rules and excessive regulation created complexity and resulted in a considerable amount of different institutional practices, which prevented transparency in the implementation of these various regulations. The most concrete consequence of this lack of uniformity was the differentiation of the procedures for the same institutions or courts. Considering this complexity, individuals argued that it was essential to unify and simplify the legal framework for regulation of the Internet.

\section{Issue Mapping}

The Working Group, which combined with the Board in order to discuss these issues, develop proposals, and solve the above-mentioned problems in the format of a draft law, held all of their meetings, interviews, discussions, and solution formulations publicly.

Participation of individuals, the public and private sector, NGOs, technical groups and academia in the Working Group was encouraged by the Board. ${ }^{9}$ To broaden and increase participation, the Working Group made announcements before meetings both on the Internet and in visual and written media. In addition, after all meetings the Working Group made it possible

\footnotetext{
${ }^{9}$ www.internetkurulu.org.
} 
for all stakeholders within and outside the Working Group to be informed about decisions made at the meeting and express their opinions over multiple communication platforms, such as social media and the webpage of the Board.

The Working Group conducted all procedures in a transparent, accountable, and open format. Every step was traceable over the webpage of the Board. The aim of this initiative was to prepare and submit an amendment text concerning Law 5651 to the Parliament, on which all stakeholders would agree.

The mission and scope of the work of the Working Group was to prepare a draft Law regarding Law 5651 in accordance with common suggestions and opinions of all stakeholders, to submit that draft Law to the Parliament, and to follow-up on its legislative procedure.

All stakeholders, namely national and international private ICT companies, governmental institutions such as $I T C A, T I B$, the Ministry, NGOs, academia, technical groups, and individuals, involved in the Board developed a joint mission for addressing the barriers created by Law 5651

Core functions of the Working Group in pursuing a fully transparent workflow:

- to determine problematic provisions in Law 5651 in line with the opinions and suggestions of related stakeholders,

- to propose solutions with all stakeholders,

- to create a Law which would protect a balance between the fundamental rights of the citizens and the freedom of doing business on the Internet

\section{B. Organizational Model and Structure}

The Communique on the Internet Improvement Board structured and informed the Board. ${ }^{10}$ According to the Communique of the Board, all seven members appointed by the Ministry had a 2-year term and election criteria were designed to represent all stakeholders within the Board.

In the Working Group, all stakeholders were participants. There were no selection criteria or procedures in practice, which was a deliberate choice to ensure a flexible forum for discussion and work.

Following a multistakeholder approach, the Ministry prepared its Communique. Before publishing it in the official gazette, the Ministry received opinions from different stakeholders. So that the draft communique was shared with all stakeholders, it was published online and was distributed electronically prior to publication. The framework was shaped by the comments provided by different stakeholders, which determined its final form. The decision making method of the Board and the Working Group was either voting or consensus/rough consensus.

The Board is based in Ankara and İstanbul. The Working Group was based in İstanbul. Except governmental institutions, most of the other stakeholders were based in İstanbul. Therefore the Working Group preferred to organize their meetings in İstanbul in order to increase and make stakeholder participation easier. There is also an important virtual component of the Board: www.internetkurulu.org. The Working Group also used this website for their work. By using this

\footnotetext{
${ }^{10} \mathrm{http} / / /$ www.resmigazete.gov.tr/eskiler/2013/12/20131214-4.htm.
} 
website the Board provided transparency regarding the Working Group's work, and enabled a constant information flow between stakeholders and others outside of the Working Group. By using technologies that enabled remote participation, such as instant messaging, e-mails etc., the Board could centralize the structure of the Working Group.

One of the core components and fundamental rules of the Board is equity of all stakeholders in the operation. There is no preference for one stakeholder over others and all have an equal voice within that context. In that regard, the Board does not discriminate between the stakeholders in terms of their status, organization, or whether they are representing the private sector or are an NGO. In order to ensure equality, the transparency of the Board's activities are enhanced by publishing all agendas in advance and online, sharing the comments provided by each stakeholder online, and publicizing decisions online. In that way, a safeguard is established to avoid any discriminatory behavior. It is noteworthy that the Ministry only gives secretariat and administrative services to the Board. Besides that support, the Board coordinates everything in collaboration with all stakeholders.

When it comes to the decisions within the Board, considering the nature of the Board as a counseling body, a rough consensus approach is adopted and applied depending on the merits of a specific case, which gives the Board flexibility and a broad area to maneuver.

There were hybrid support models in order to financially sustain the Working Group. One of them was financial and logistical support from Universities in terms of meeting places, providing accommodation, and other technical and logistic support. The other main financial resource came from NGOs and private sector companies. They assumed particular organizational expenses of meetings. The Board worked in collaboration with all stakeholders to financially sustain the DG, too. On the other side, the Ministry partly supports the Board financially.

\section{Participation}

Academia, civil society, private and public sector institutions, technical groups, and individuals were stakeholders in the Working Group. ${ }^{11}$ The Board gave importance to the idea that each stakeholder was represented within the Working Group and created a distributed group to accomplish this. Another important principle that the Board pursued during its joint work was providing each stakeholder an equal voice.

Stakeholders played an important and active role in the following ways:

- Identifying legal and technical problems of Law 5651,

- Analyzing pros and cons of Law 5651,

- Discussing details of legal and technical problems taking into consideration the experience of related stakeholders regarding Law 5651 in practice,

- Hearing think tanks, informatics NGOs, and Universities, which already conducted SWOT analysis as to Law 5651,

- Determining problems in court practices concerning Law 5651 through the help of judges and prosecutors,

- Negotiating directly with related governmental institutions in order to show them the negative effects of Law 5651 on the ICT market, on the fundamental rights of citizens, on

\footnotetext{
${ }^{11}$ See appendix 1 for full list of stakeholders within the Working Group.
} 
the information society and network economy, and on the investment climate of the country.

Related governmental institutions mostly played the role of "listeners" rather than the legislative or regulatory role played by other stakeholders. After hearing, discussing, negotiating the identified problems and getting all points of view, approaches, objections and solution proposals, the Government explained its point of view and justifications. The Board acted sometimes as a catalyst in order to find middle ground between the Working Group and government when they couldn't agree on the solutions or formulations of determined problems. All other stakeholders within the Working Group acted together in most cases and represented common interests.

There are no specific rules, regulations, or laws that address this role distribution. But we can derive this role distribution from the Article 29, "Mission of the Internet Improvement Board", of the Decree Law of the Ministry. Article 29 envisages in broad terms that the Internet Improvement Board is responsible for creating policy and strategy proposals for the Government, which will foster the wide, effective, and easily accessible usage of the Internet in economic, commercial, and social life and also in the field of science, education, and culture. The Board is responsible for creating and submitting proposals oriented around secure, free, and independent Internet usage and to add value to the Internet. Based on that mission, the Board acts as catalyst from the very beginning between government and all other stakeholders. The government plays a more passive role and gives priority to all other stakeholders.

Within this distributed ecosystem all stakeholders had an active role in terms of expressing their opinions. There were no different tiers of participation for stakeholders within the Working Group. Additionally, in order to increase participation and inclusivity, the Working Group did not establish any prerequisites to be a part of the joint work. There are only rules for becoming a member of the Internet Improvement Board or giving up membership status. Other than that specific board, there were no specific rules for entry/exit for stakeholders from the Working Group. Participation was always open to each stakeholder who wanted to contribute on related issues. The Board did not establish specific and detailed rules for participation. Stakeholders could determine freely at any time to be a part of the Working Group. They all always had the right to bring up a new issue or problem to discuss within the Working Group.

There were a number of affiliated enablers that interacted with the Working Group, while it was seeking solution proposals. The Working Group stipulated a very flexible participation environment. Therefore there were both institutional enablers and individual enablers that interacted with the Working Group. Employee associations, think tanks, informatics NGOs, Internet journalists, student clubs, hosting companies, ISPs, and intermediaries were the main enablers for increasing participation. The main information flow between the Working Group members was the website of the Board and its mailing list. Multiple communication platforms such as Skype, Adobe Connect, instant messaging, and email were other important information structures in place. 


\section{Decision-Making Structures}

\section{Main Decision Structures}

The process by which an issue entered into the decision-making process consisted of different layers. Sometimes the Board would offer an issue for decision-making, but the government, private sector, academia, technical groups, civil society, and individuals could also bring an issue for decision-making at any time.

During the work of the Working Group, issues were identified by:

- court practices and decisions,

- practices of regulatory bodies and their decisions,

- complaints of different stakeholders regarding Law 5651 to the courts and/or regulatory bodies,

- specific research commissioned at the Parliament concerning the IT and Internet,

- meetings and consultation of the Internet Improvement Board and the Working Group to the all stakeholders,

- directly from the citizens.

For issue identification by those within the Working Group there were some processes in place, such as:

- regular meetings with all stakeholders and the Government,

- e-mail groups,

- social media activities in order to create awareness of the disadvantages of the Law 5651,

- negotiations and discussion platforms both online and face to face,

- trainings on Law 5651 for the courts,

- events such as conferences, panels, symposiums, workshops.

Academia, NGOs, think-tanks, and sometime private sector companies outside of the Working Group, also organized different kinds of events which gave the opportunity to all stakeholders to identify the issues, address them to related stakeholders, and discuss possible solutions as to Law 5651 which support the work of the Working Group.

After identifying the problems mentioned above, the Working Group requested that relevant stakeholders who faced problems concerning Law 5651 prepare grounds and justifications for their specific problems. Within this context, relevant stakeholders then submitted legal, technical, and economic statistics to the Working Group that illustrated the adverse impacts of Law 5651. Before making any decision, it was also important to the Ministry to listen to all stakeholders, hear their reasoning, and discuss together the impact of problematic provisions of Law 5651. Based on those concrete facts and findings, the Working Group started to formulate solutions with the help of all stakeholders within and outside of the Working Group. Judges, prosecutors, the Ministry, the Information Technologies and Communication Authority, and the Telecommunication and Communication Presidency were all indispensable parts of this solution formulation. Upon an official invitation of the Board, these officials became involved in the process. They pointed out the practical problems of Law 5651 that they faced and struggled with. Their contribution was very important in terms of formulating new provisions for Law 5651 based on that practical experience. 
The decision-making structure within the Working Group was designed to find and reach a solution collaboratively. The Working Group decided either by consensus or by majority on issues. There was also no centralized institution or primary decision maker. All stakeholders had equal footing in the decision-making process. There was not a professional staff that supported the work of the decision makers, but the Ministry did provide secretarial and administrative support to the Board.

\section{2. $\quad$ Supporting Decision Structures}

To assist the Board and Working group in their decision-making processes, there were two additional groups that participated where needed.

i. $\quad$ Enablers

The Working Group utilized following enablers:

- Expert groups,

- Think tanks,

- Discussion forums,

- Websites,

- Mailing groups,

- Social media activities

- Internet Media companies,

- Blogs

The Working Group identified the related enablers for a particular issue as follows. The Working Group took the specific needs of a particular issue, determined the parameters for it and, according to these facts and findings, selected appropriate enablers for the particular issue. Enablers helped the Working Group on a specific topic relevant to their experience and knowledge. The Working Group worked closely with the relevant enablers and undertook analysis, prepared proposals, reports, projects, and found and evaluated solutions before announcing them to other stakeholders. Sometimes there was an overlap between stakeholders and enablers.

According to the Communique of the Board, relevant enablers are an important part of the governance and working model of the Board. Therefore the Working Group can call on the relevant enablers at any time in order to clarify issues that require specific knowledge. As a result of this structure, enablers provided input to the Working Group constantly, increased participation, and created awareness within their community on the related issue.

The most important expert dialogue was TEPAV, ${ }^{12}$ a think tank, which supported the Working Group. TEPAV is a think tank based in Ankara, Turkey. It was founded by a group of business people, academics, and bureaucrats in 2004 with the help of the Union of Chambers and Exchanges of Turkey (TOBB). TEPAV aims to contribute to policy design and enrich the knowledge content of economic discussions in Turkey. TEPAV's staff is composed of professionals who are experts on their respective fields. TEPAV's board members are directors of private sector organizations, senior academics, and bureaucrats. TEPAV is a private and nonpartisan think tank. It has strong ties to TOBB, the umbrella organization of all private sector

\footnotetext{
${ }^{12} \mathrm{http}: / /$ www.tepav.org.tr/tr/.
} 
businesses of Turkey. TEPAV is committed to providing fact-based inputs for agencies designing and implementing economic policy.

\section{ii. $\quad$ Other Working Groups}

During the course of its operation, the Working Group attempted to disentangle technical and non-technical issues. In addition to requiring a broad range of participation and collaborative work from different stakeholders, the Working Group also required the help of existing working groups, which were already operating under the Board. Each working group consisted of people who had experience on, background in, and knowledge of technical and non-technical issues. For example, the "Working Group for Legislation" was composed of lawyers and academic persons from the Universities etc. The "Working Group for Standards and other Technical Issues" consisted of mostly IT specialists, IT engineers, standardization bodies, technical committees such as ISACA ${ }^{13}$, and IT departments of public and private institutions, etc., in order to tackle technical issues. The Working Group combined each working group's facts and findings together at public (or sometimes closed-door) meetings, and tried to deal with technical and non-technical issues in cooperation with related stakeholders within and outside of the working groups. The reason for sometimes conducting closed door meetings was to discuss and analyze issues intensively with only experts on the topic. It was always easy to solve technical problems. Referring a related standard or technology to the relevant group could solve problems in a short period and without significant efforts. However, when it came to non-technical issues and if there was a need to make new law or amend current legislation, it was more cumbersome and bureaucratic. This is because legislation entails all stakeholders with technical and non-technical backgrounds to find alternative solutions concerning the issues.

The Working Group benefitted from utilizing other working groups. When each working group finished identifying issues, the Working Group organized meetings aimed at incorporating inputs from both technical and non--technical participants.

\section{E. Outcomes}

In line with their stated mission and goals, the Working Group was truly successful concerning the collaborative work which we explained in this case study.

The Working Group solved problems regarding Law 5651 using the principles below:

- Collaborative working,

- Balancing the power of the Government and the voice of all other stakeholders,

- Bridging information flow,

- Nexus for all stakeholders,

- Without exception, hearing all stakeholders within and outside of the Working Group,

- Allowing wide participation, both online and onsite,

- Respecting each stakeholder's rights,

- Noting all problems and sharing them with the public, including the problems or issues that were not resolved. In this way, even if no consensus was achieved in the short term for a specific matter, the stakeholders' main concerns were easily tracked and the stakeholders knew the matter will be on the agenda in the medium or long term.

\footnotetext{
${ }^{13} \mathrm{https}$ ://www.isaca.org/Pages/default.aspx.
} 
Because the request to work "collaboratively" came from the "bottom up" all stakeholders were willing to work closely in order to reach a consensus. "Hearing" each stakeholder was the key factor of success. The Working Group moderated sessions fairly in order to create a sound environment of discussion. During all meetings there were no limitations on sharing discussions on social media platforms. Through these platforms other stakeholders outside of the Working Group could keep informed about the issues discussed, indirectly participating in the meetings and sharing their opinions. Therefore, there were no obstacles to reaching a solution. The work conducted by the Working Group was exactly a "balancing the power of the Government and the voice of all other stakeholders". Within the Working Group, even the government was one of the stakeholders and had an equal voice like all of the others. This approach made it possible to create a balance amongst the stakeholders.

The organization worked as planned. One reason for this success was that the Working Group was established at right time to allow for widespread participation. When the Working Group finished preparing amendments to Law 5651, it submitted these with the Board to the Ministry in order to start the legislative process. The Board and the Working Group then followed up with the prepared amendments before the Parliament with the Ministry. During negotiations in Parliament, both the Board and stakeholders within and outside the Working Group joined sessions in order to flesh out collaborative solutions. Parliament took into consideration the Working Group's “amendments” when they accepted changes to Law 5651.

Academia, civil society, private sector, and even individuals were the most effective stakeholders in the above process, offering creative and reasonable resolutions. At the end of this joint work, all stakeholders were mostly happy with the solution. Because of this common consensus amongst stakeholders, other parties also accepted the resolution of the Working Group.

There are a number of factors that led to acceptance and implementation of the resolution of the Working Group:

- Transparency of the working and preparation process,

- Respect for each stakeholder's right to have an equal voice,

- Respect for the need to protect fundamental rights such as freedom of expression, freedom of information, right to access, and data protection, and the needs of the industry to reach information society and network economy,

- Continuous information flow amongst stakeholders even outside of the Working Group,

- Collaborative working procedure in order to address determined goals,

- Openness to listen and evaluate opinions of other national/international stakeholders besides the Working Group,

A key part of this openness and transparency was the use of technology to enable remote access to meetings, as well as e-mail and instant messaging tools used to enhance inclusiveness and participation. During meetings there is also the possibility of commenting on discussions over social media websites. These free tools helped the Working Group identify solutions. The important thing was to bring different point of views and different approaches to the table that made it easier to identify solutions. The Working Group used broadcasting and/or webcasting technologies in order to reach more stakeholders outside of the DG and encourage them to actively join the debates using social media platforms and/or e-mail. Over the Working Group's website, all stakeholders outside of the DG could easily reach out to all group members and 
share their opinions, as well as offer new issues for discussing. In order to create and work on a joint document, the Working Group used crowd-sourcing technologies such as Google Drive. These tools stimulated participation from a wider range of stakeholders.

Because of this strong basis, which was fundamentally shaped by decentralized, distributed, collaborative Internet governance rules, the resolution of the Working Group was long lasting. The most important indicator of this is that a few months after releasing the Working Group resolution, the Ministry took this resolution up as the basis of its legislative work on Law 5651. Political parties other than the government party and specific Parliamentary committees also accepted the resolution for their legislative work at the Parliament. The stakeholders we interviewed also mentioned that when they worked together and created a text collaboratively, it made the resolution strong and long lasting because each of them could easily follow-up on the issues addressed in this joint work.

The Working Group and its resolution showed that many kinds of problems relating to the Internet could be solved in collaboration with all stakeholders both within and outside of the Working Group.

As a result of that joint work, the following changes were made and new Articles were added to Law 5651, mostly in accordance with the concerns raised by the relevant stakeholders during the Working Group:

- The provisions that permitted the use of DNS blocking and were hurdles to the promotion of freedom of expression were revised. In this regard, it has been clarified that Internet content can only be blocked by using the URL restriction method. Under the new rules, judges can only apply the DNS technique in exceptional circumstances and the law requires the judges who apply this method to lay down their justifications explicitly. This reform is a breakthrough for promoting freedom of expression, which reflects the concerns that were put forward by the Working Group during the stakeholder consultation process.

- Furthermore, a new rule was introduced to Law 5651 regarding the restriction of access to websites. According to this new rule, if blocking access is considered to accomplish the aim, this decision can be applied for a limited time. The aim of this rule is to avoid any adverse outcomes of long-standing restrictions, which are temporary injunctions in nature but which, due to the lack of safeguards, could evolve into punishments. The restriction of web sites with no time limit was one of the most criticized aspects of Law 5651.

- The criminal liability provisions for content, hosting, and access providers were reformed and the criminal liability was converted to pecuniary liability. In this regard, provisions that could lead to imprisonment were abolished; a relatively better balance was established on the liability of Internet content, hosting and access providers.

- Considering the concerns with regard to the fragmentation of institutional practices of implementation, a new institutional body was established, the so-called Access Providers Union, which is in charge of carrying out the implementation of restriction decisions. 
- In accordance with the concerns and criticism, Law 5651 also established a delicate balance between freedom of expression and protection of personal rights (including privacy) and added a new rule enabling the restriction of access to illegal content infringing on personal rights (including privacy) on the URL basis.

According to the interviews we conducted with related stakeholders, all agreed to continue to work in a multistakeholder fashion regarding Internet-related issues as a result of the above collaborative success. Stakeholders agreed that lack of multistakeholderism could result in a disparity amongst stakeholders, and lead to disproportionate Internet regulations in terms of the needs of the ICT sector vs. the protection of individuals. Stakeholders highlighted the importance of having an equal voice within the IG ecosystem. They pointed out that the only way to have an equal voice is acting as a Distributed Group. They also mentioned that when they considered the current amendment procedure of Law 5651, which was done solely by the Government itself without asking any stakeholders, even the Board, illustrative of the importance of multistakeholder Internet governance, transparency, and multiple voices.

\section{F. Observations and Lessons Learned}

Key elements of the success of the Working Group are: Pluralism, Collaboration, Multiple Voices, Transparency, Widespread Participation, Keeping Communication Channels Alive, Balancing Needs, and Establishing a Common Language between stakeholders.

Lessons learned from that experience are as follows:

- The importance of regulatory impact assessment with all stakeholders before submitting a law or regulation to the parliament: Law 5651 was prepared and came into force without conducting a regulatory impact assessment with stakeholders. As a result of that fact, Law 5651 started to create difficulties in practice and the need to amend Law 5651 became apparent in a very short period. Different stakeholders expressed their complaints and opinions on the problematic provisions of Law 5651. In order to solve these problems the Board decided to combine all stakeholders' energy and efforts together. Based on this strong bottom up request, the Board organized the aforementioned collaborative work. At the end of that joint work all stakeholders agreed upon new provisions that had been analyzed in depth in terms of their effects on related stakeholders. Therefore we could call this collaborative work a kind of "impact assessment" and "solution mapping and formulation".

- Need for a platform that represents all stakeholders: The Board, pursuant to its multistakeholder character, incorporated all stakeholders and represented the voices of each group. Such a platform provided all stakeholders a venue to express their points of view easily and directly, to use their time efficiently and effectively, to combine their voices and power together, to reach and work with the government as a stakeholder directly, and to strengthen their agenda before related institutions such as Parliament, Ministries, etc.

- Importance of transparency of work, processes, and procedures: The Board envisaged a fully transparent work environment, processes, and procedures in order to make joint work fruitful. 
- Importance of constant information flow and collaborative work of all stakeholders: During the entire process, the Board gave importance to continuous information flow amongst stakeholders. The Board organized meetings in order to foster collaborative work and speed up the procedures.

If another organization/country/region/group wanted to build upon the experiences of the Working Group in their own organizations, some key observations are:

The limited number of members of the Board made its work much easier, particularly in terms of meeting and working with all stakeholders. Because each member represented a different stakeholder, all stakeholders in the country had an equal and strong voice within the Board. The Board worked under the Ministry, but acted totally free in its capacity. This connection to the Government gave to the Board power to follow-up on its agenda before the Parliament and related governmental bodies. Taking into consideration the slowness and bureaucracy of the legislative process of the Parliament, accessibility to the government allowed the Board to speed up the legislative procedures of Law 5651. Their close relationship with the government also helped overcome bureaucratic hurdles. Through the Board, all stakeholders within and outside of the DG also had very quick access to submit their requests and problems to the Government. On the other side, Government had a very useful advisory tool when they need to answer and solve Internet related questions and matters. The Board also had the ability to set up different working groups on related topics, which enabled them to gather all stakeholders under a single roof. The Board could also get consultancy from related stakeholders in order to deal with specific problems for particular stakeholders.

The necessary steps that should be taken by another organization/country/region/group are specified below:

Limit the number of the members of the Board: In order to ensure flexibility within the Board and speed up the dialogue and communication with stakeholders, the number of Board members should be limited. Turkey had prior Internet Board experience. The first version of the Board consisted of exactly 37 people $^{14}$, who represented different stakeholders. This model created difficulties in regards to holding regular meetings, creating a convenient discussion platform, evenly distributing the workload between members, and so forth. Meetings with large numbers of stakeholders took longer than planned in order to follow up on envisaged actions, projects, and start to discuss new developments and suggestions. There were a lot of working groups under that Board and one related member moderated each of them. The working groups consisted mostly of outside stakeholders. Depending on the time and devotion of the moderator, some of working groups were successful, but most of them were inactive, which negatively affected related projects and the work of the Board. Because all projects were assigned to one or more related working groups, when these groups were ineffective this affected directly the agenda of the Board. In order to avoid this kind of issue, we suggest limiting the number of members.

Give importance to the fact these members are representing all stakeholders: It's important to give a place and provide equal voice to each stakeholder within the DG. Therefore, individuals

\footnotetext{
${ }^{14}$ http://www.ubak.gov.tr/BLSM_WIYS/INT_KRL/tr/Belgelik/20100721_094452_40723_1_64.html.
} 
should take this fact into consideration when they nominate the members from each stakeholder group.

Create an autonomous and independent Board: One of the core components when setting up a Board is to ensure its autonomy and independence. This rule will underpin the interdependent and transparent workflow of the Board. It's also a prerequisite for respecting fundamental rights.

Put into practice the key rules to follow and fundamental rights with respect the Board: We call this the "nuncupative" working framework or codes of conduct of the Board. It may vary from country to country in some respects, therefore, there is no tailor-made framework. However, every board should have fundamental rights of the stakeholders regarding Internet related matters. From the point of view of the Board, we develop our nuncupative codes of conduct based on national and international norms, conventions, reports, and court decisions. Showing trends, new developments, approaches, problems, and solutions relating the Internet, these codes impact the Board's agenda, priorities, projects, and plans directly.

Create transparent and multiple communication channels in order to make possible information flow between the Board and the all stakeholders to facilitate work between all stakeholders.

Follow-up mechanisms and methods for the stakeholders from the Board.

Accountability of the Board and all other stakeholders in respect to the joint works.

In building upon the experiences of the Board and Working Group, another organization/country/region/groups should avoid the following:

- Setting up the Working Group with low participation,

- Holding closed-door meetings even with participation limited to predetermined stakeholders within the Working Group,

- Publishing all decisions, works etc. only for members of the Working Group,

- Working only with members of the Working Group.

We observe that there were a number of helpful tools for the Board and Working Group, such as:

- tools which provide and promote transparency,

- tools which provide continuous communication,

- tools which enable collaboration,

- tools which promote wider participation, such as remote participation, crowdsourcing working techniques, etc...

- tools which are the most accessible in the local/national context,

- tools which enable participants to understand each other better and balance the needs of different stakeholders.

Another organization/country/region/group should also consider creating such instruments, which have a significant impact and help the work of the Working Group:

- They should create on-going and/or ad hoc working groups on topics which require more specific experience and/or intensive time to analyze, 
- They should not avoid seeking consultation from local, regional or international institutions on related issues, if needed,

- They should create online platforms which provide free flow of information, which increases remote participation, and which strengthens transparency amongst all stakeholders within or outside the Working Group. 